\title{
Einfluss von Radverkehrsaufkommen und Radverkehrsinfrastruktur auf das Unfallgeschehen
}

Dankmar Alrutz Wolfgang Bohle Reinhold Maier Markus Enke Maria Pohle Frank Zimmermann Jörg Ortlepp Marcel Schreiber

Unfallforschung der Versicherer

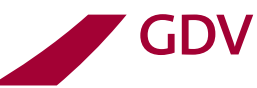




\section{Einfluss von Radverkehrsaufkommen und Radverkehrsinfrastruktur auf das Unfallgeschehen}

Dipl.-Ing. Dankmar Alrutz

Dipl.-Ing. Wolfgang Bohle

Prof. Dr.-Ing. Reinhold Maier

Dipl.-Ing. Markus Enke

Dipl.-Ing. Maria Pohle

Dipl.-Ing. Frank Zimmermann

Dipl.-Ing. Jörg Ortlepp

Dipl.-Ing. Marcel Schreiber 


\section{Impressum}

Gesamtverband der Deutschen Versicherungswirtschaft e. V.

\section{Unfallforschung der Versicherer}

Wilhelmstraße 43/43G, 10117 Berlin

Postfach 0802 64, 10002 Berlin

E-Mail: unfallforschung@gdv.de

Internet: www.udv.de

Facebook: www.facebook.com/unfallforschung

Twitter: @unfallforschung

YouTube: www.youtube.com/unfallforschung

ISBN-Nr.: 978-3-939163-57-2

Redaktion: Dipl.-Ing. Jörg Ortlepp, Dipl.-Ing. Marcel Schreiber

Bildnachweis: UDV und siehe Quellenangaben

Erschienen: 01/2015 


\section{Einfluss von Radverkehrsaufkommen und Radverkehrsinfrastruktur auf das Unfallgeschehen}

bearbeitet durch:

Planungsgemeinschaft Verkehr, PGV-Alrutz

Dipl.-Ing. Dankmar Alrutz

Dipl.-Ing. Wolfgang Bohle

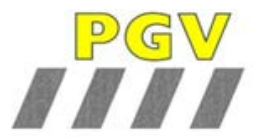

Technische Universität Dresden

Fakultät Verkehrswissenschaften

Institut für Verkehrsplanung und Straßenverkehr

Lehrstuhl für Straßenverkehrstechnik

mit Fachbereich Theorie der Verkehrsplanung

Prof. Dr.-Ing. Reinhold Maier

Dipl.-Ing. Markus Enke

Dipl.-Ing. Maria Pohle

Dipl.-Ing. Frank Zimmermann

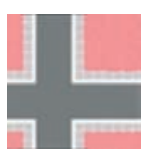

Bei der UDV betreut von:

Dipl.-Ing Jörg Ortlepp Dipl.-Ing Marcel Schreiber

Unfallforschung der Versicherer 



\section{Inhalt}

Inhalt

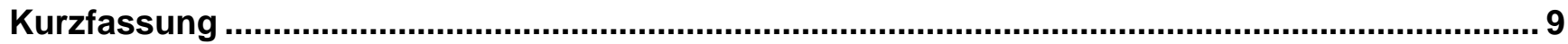

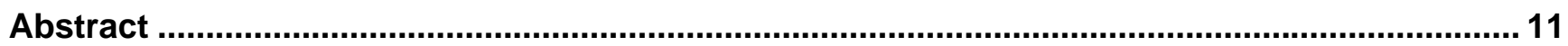

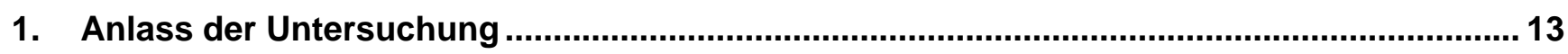

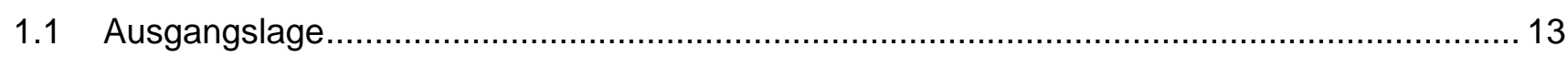

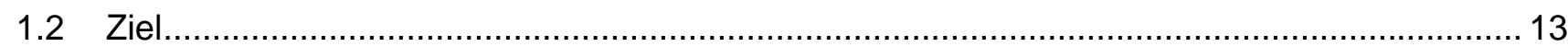

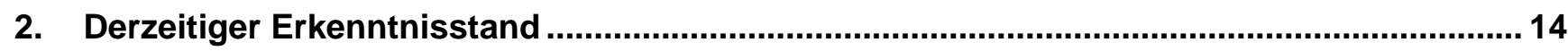

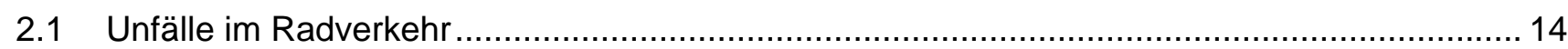

2.2 Ausprägungen des Unfallgeschehens: besonders gefährdete Radfahrer-Gruppen............... 16

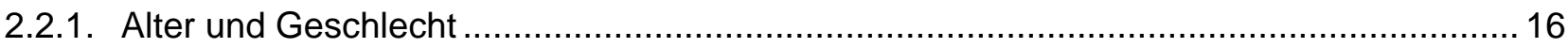

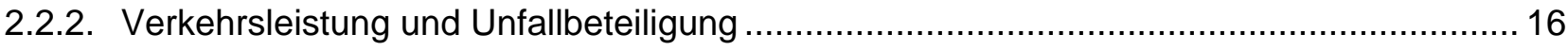

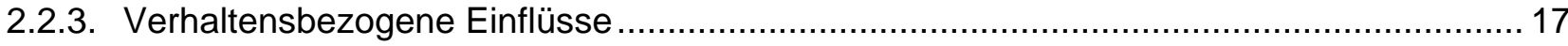

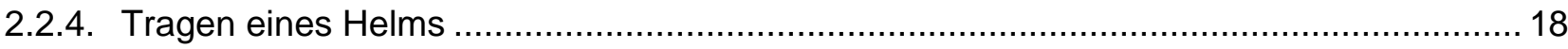

2.2.5. Nutzung von Elektrofahrrädern (Pedelecs 25 und Pedelecs 45)................................ 18

2.3 Unfallgeschehen auf Streckenabschnitten mit nicht lichtsignalgeregelten

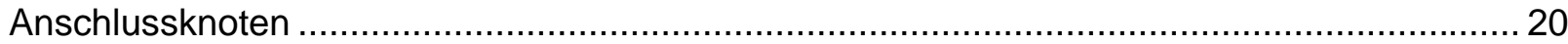

2.3.1. Radverkehrsführung auf Radwegen, Radfahrstreifen und Schutzstreifen ...................... 20

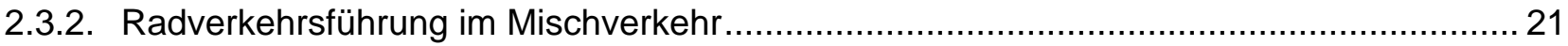

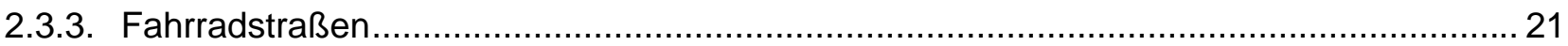

2.4 Radfahrer-Unfälle an Knotenpunkten mit Lichtsignalanlage ............................................ 21

2.5 Geschwindigkeiten des Radverkehrs und geschwindigkeitsbedingte Verletzungsschwere ..... 24

2.5.1. Geschwindigkeiten des Radverkehrs ................................................................. 24

2.5.2. Kollisionsgeschwindigkeit und Verletzungsschwere ................................................. 25

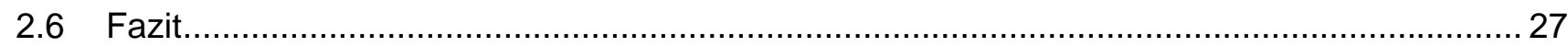

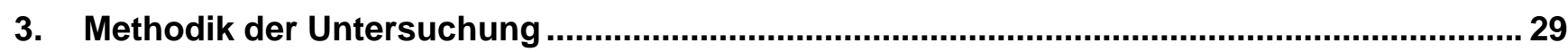

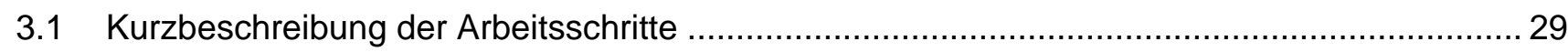

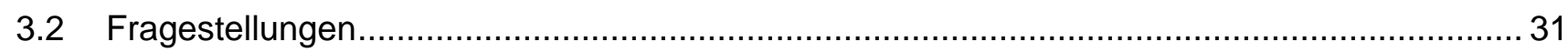

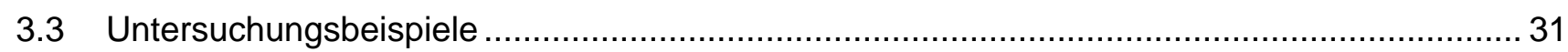

3.4 Ermittlung von Stärken, Zusammensetzung und Verhaltensmerkmalen des Radverkehrs ..... 33

3.4.1. Zählungen und Verhaltensmerkmale............................................................. 33

3.4.2. Verhaltensbeobachtungen an lichtsignalgeregelten Knotenpunkten........................... 33

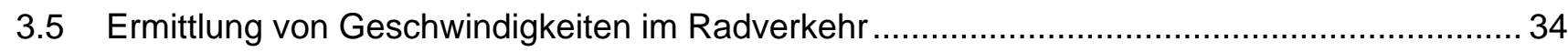

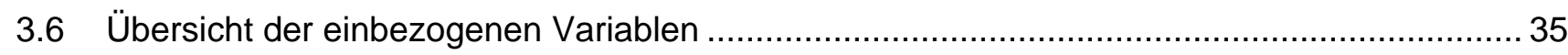

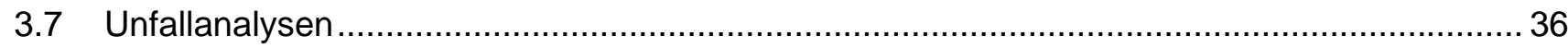


3.7.1. Makroskopische Unfallanalysen - Kenngrößen der Radverkehrsunfälle für Streckenabschnitte und Knotenpunkte

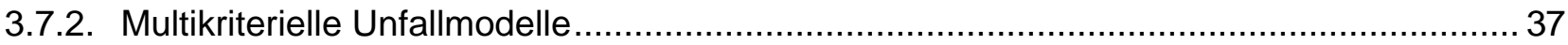

3.7.3. Vertiefungsanalyse kleinräumiger Einflussgrößen (mikroskopische Unfallanalyse).......... 38

3.8 Künftige Entwicklung des Radverkehrs und der Radverkehrs-sicherheit............................ 38

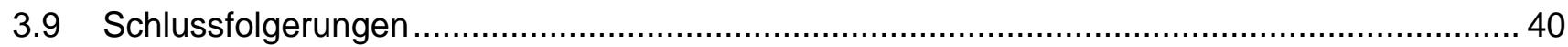

4. Einfluss von Radverkehrsstärken, Alter und Geschwindigkeiten von Radfahrern auf

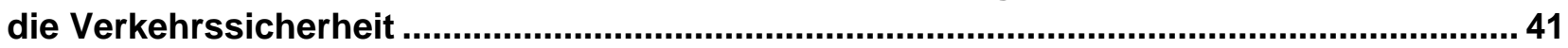

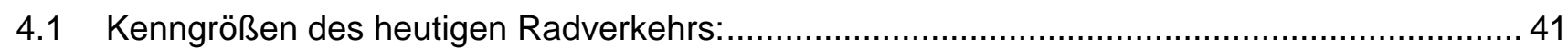

4.1.1. Altersgruppen im Radverkehr und genutzte Fahrradtypen...................................... 41

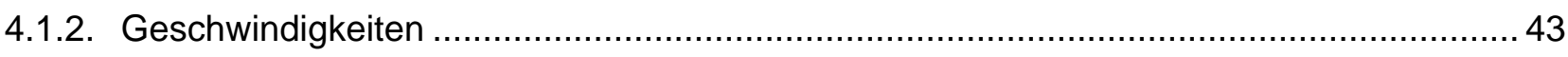

4.1.3. Fahrverhalten an lichtsignalgeregelten Knotenpunkten .......................................... 49

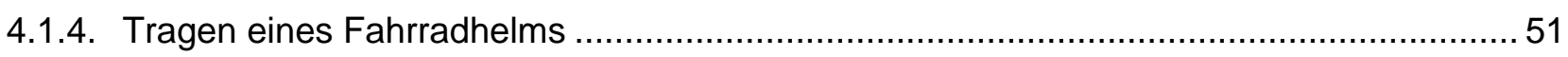

4.1.5. Verteilung der Radfahrerunfälle im Straßenraum.................................................. 56

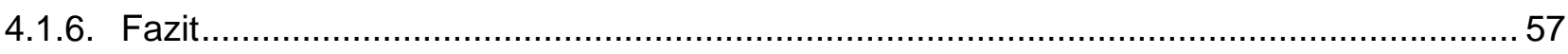

4.2 Radverkehrsstärken, Unfallgeschehen und Regelbefolgung .......................................... 57

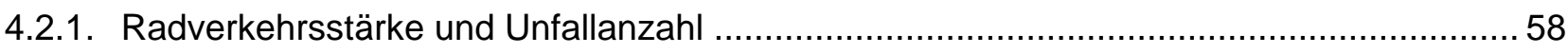

4.2.2. Verkehrsanteile und Unfallbeteiligung des Radverkehrs in ausgewählten Städten

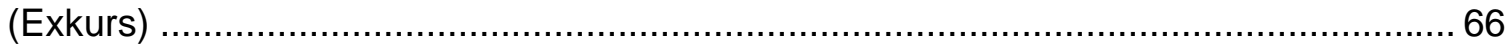

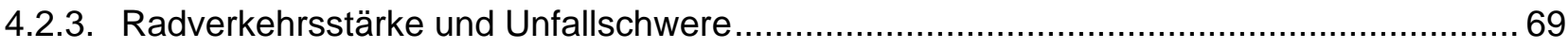

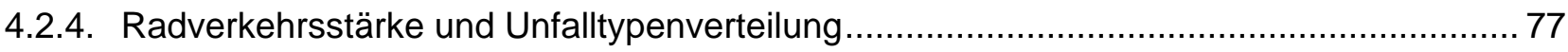

4.2.5. Zusammenhänge zwischen Radverkehrsstärke und Regelbefolgung .......................... 82

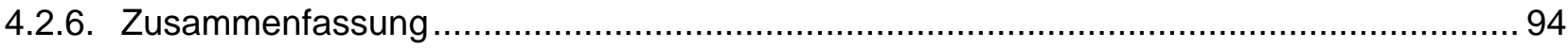

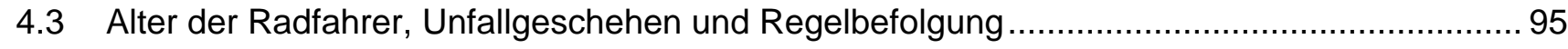

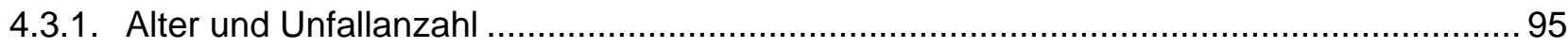

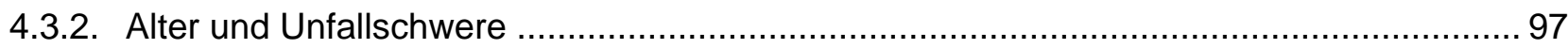

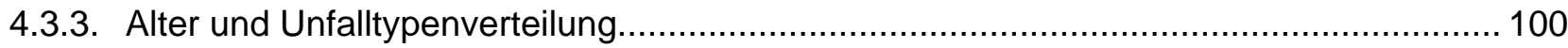

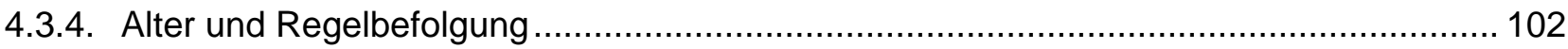

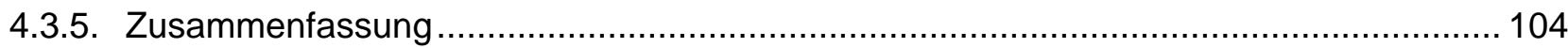

4.4 Radfahrergeschwindigkeiten, Unfallgeschehen und Regelbefolgung .............................. 105

4.4.1. Radfahrergeschwindigkeiten und Unfallanzahl .................................................. 105

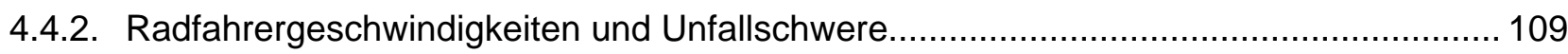

4.4.3. Radfahrergeschwindigkeiten und Unfalltypen .................................................... 111

4.4.4. Radfahrergeschwindigkeiten und Regelbefolgung ........................................... 114

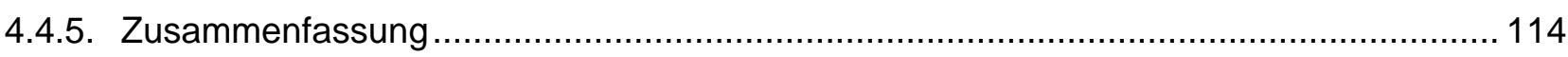

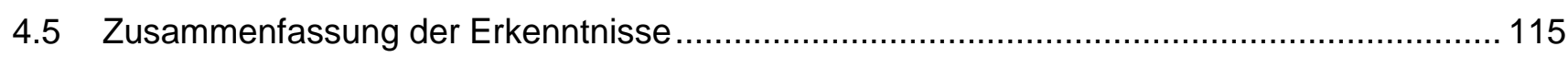




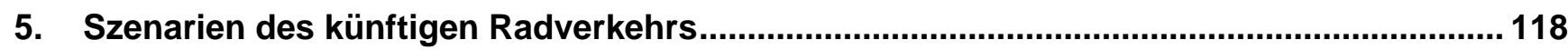

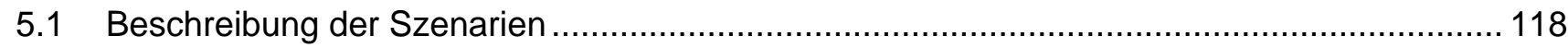

5.2 Auswirkungen der Szenarioannahmen auf die Verkehrssicherheit .................................. 123

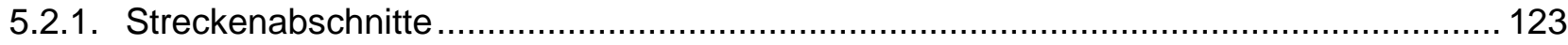

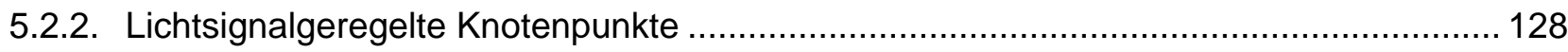

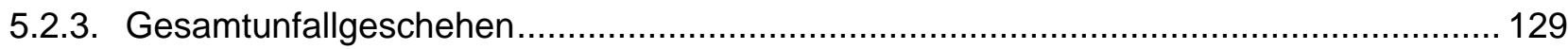

5.3 Ergebnisse der Szenarienberechnungen ................................................................ 130

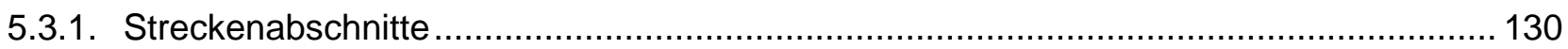

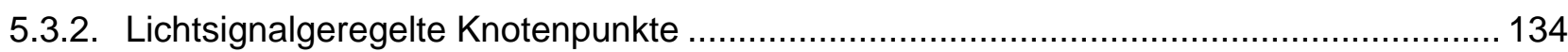

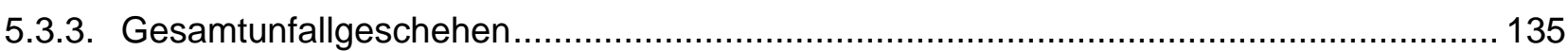

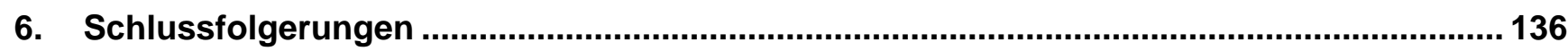

6.1 Folgerungen im Hinblick auf steigende Radverkehrsstärken (Szenarien S1, S2-A-20, S2-

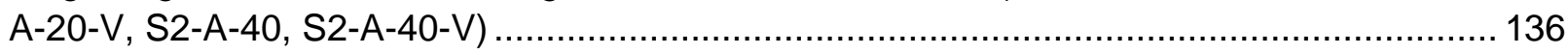

6.1.1. Streckenabschnitte mit Einmündungen und Grundstückszufahrten.......................... 136

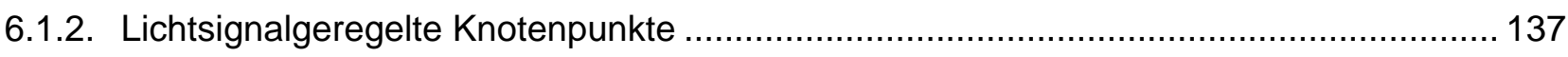

6.2 Folgerungen im Hinblick auf die Veränderung der Altersstruktur (Szenariengruppe S2-A) ... 138

6.2.1. Streckenabschnitte mit Einmündungen und Grundstückszufahrten ............................ 138

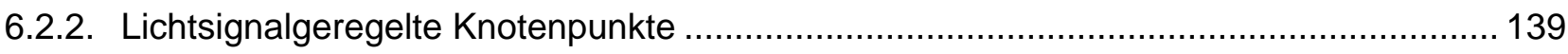

6.3 Folgerungen im Hinblick auf steigende Radverkehrs-Geschwindigkeiten (Szenarien S2-

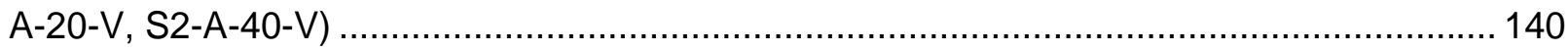

6.3.1. Streckenabschnitte mit Einmündungen und Grundstückszufahrten ............................. 140

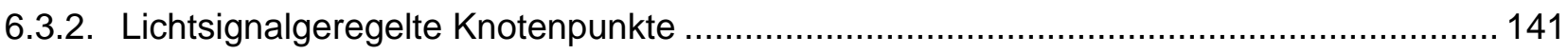

6.4 Ergänzende szenarienübergreifende Folgerungen .................................................. 142

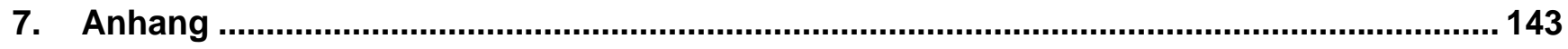

7.1 Theorie der Verallgemeinerten linearen Modelle ........................................................ 143

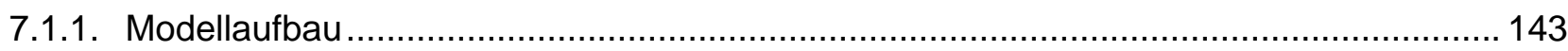

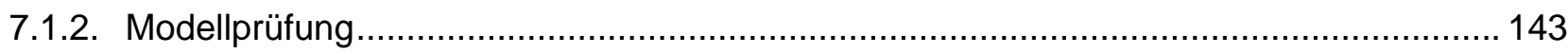

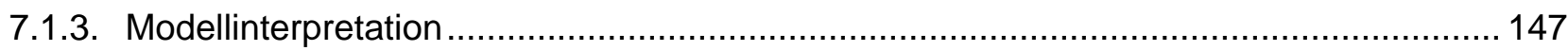

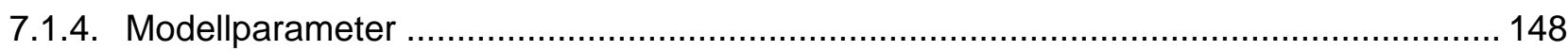

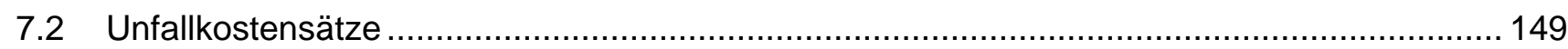

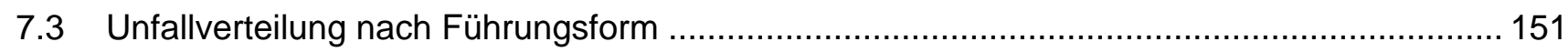

7.4 Unfalltypenverteilung an Strecken inklusive Unfalltyp „Sonstiger Unfall“ .......................... 153

7.5 Unfalltypenverteilung an Knotenpunkten inklusive Unfalltyp „Sonstiger Unfall“ ................... 157

7.6 Bedingte und separate Freigabe an Knotenpunkten .................................................. 158

7.7 Berechnung der durchschnittlichen Abweichungen der Radfahrergeschwindigkeiten ........... 160

7.8 Fahrlinien von Radfahrern an lichtsignalgeregelten Knotenpunkten .................................. 161

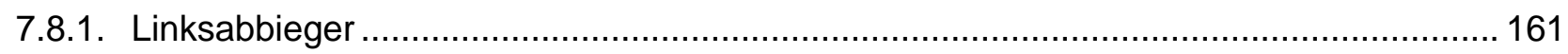




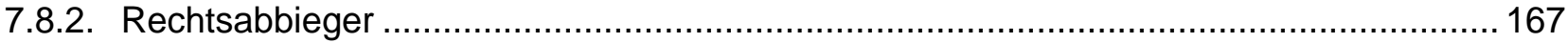

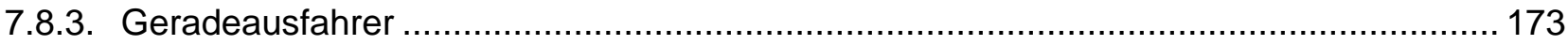

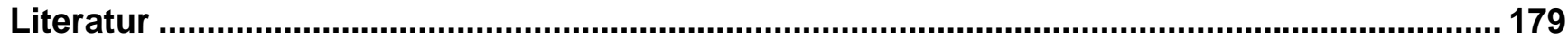

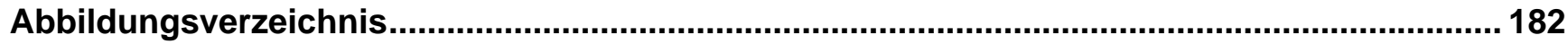

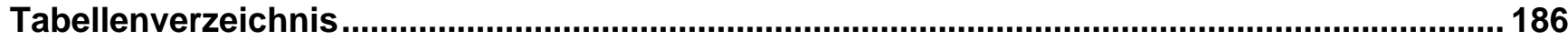




\section{Kurzfassung}

Durch den demografischen Wandel, die zunehmende Verbreitung von Pedelecs sowie die in mehreren Städten stark gestiegenen Anteile des Radverkehrs am Gesamtverkehr unterliegen die Stärken, Zusammensetzungen und Geschwindigkeiten des Radverkehrs derzeit einem erheblichen Wandel. Künftig werden insbesondere im Stadtverkehr zunehmende Radverkehrsstärken und stärker differenzierte Geschwindigkeiten von Radfahrern erwartet. Die Studie sollte klären, ob sich daraus ein Einfluss auf das Unfallgeschehen ergeben wird und welche Maßnahmen zur Verbesserung der Sicherheit des Radverkehrs zu ergreifen sind.

Die Untersuchung sollte zunächst für den heutigen Radverkehr bei unterschiedlichen Radverkehrsanlagen, Einflüsse aus Radverkehrsstärken, Radverkehrsgeschwindigkeiten und der Altersstruktur auf die Anzahl, die Schwere und die Typen von Radfahrer-Unfällen ermitteln. Aus Verkehrszählungen, Verhaltensbeobachtungen, Geschwindigkeitsmessungen und Unfallanalysen für Radfahrer ergaben sich folgende wesentlichen Erkenntnisse:

- Die mittleren Geschwindigkeiten des Radverkehrs haben sich gegenüber früheren Untersuchungen um etwa 1,5 km/h erhöht und liegen nun bei etwa $18,2 \mathrm{~km} / \mathrm{h}$.

- An Streckenabschnitten mit Anschlussknoten bedingten höhere Radverkehrsstärken mehr Unfälle und höhere Anteile von Unfällen mit schwerem Personenschaden. An lichtsignalgeregelten Knotenpunkten konnten keine Zusammenhänge zwischen Radverkehrsstärken und Unfallbelastungen bzw. der Unfallschwere ermittelt werden.

- Bei höheren Radverkehrsstärken verschoben sich die Unfalltypen an Streckenabschnitten von Abbiegen- und Einbiegen-/ Kreuzen-Unfälle hin zu Unfälle im Längsverkehr und Unfälle durch ruhenden Verkehr. Besondere Sicherheitsprobleme bestanden durch das regelwidrige Linksfahren und vor allem bei Führung des Radverkehrs auf Radwegen.

- An Streckenabschnitten hatten Über-65-Jährige ein etwa doppelt so hohes mittleres Unfallrisiko wie 25- bis 65-Jährige. An lichtsignalgeregelten Knotenpunkten konnte kein Zusammenhang zwischen dem Alter der Radfahrer und der Anzahl der Unfälle festgestellt werden.

- An Streckenabschnitten traten bei Unfällen älterer Radfahrer anteilig häufiger schwere Unfälle auf. Ein ähnliches Bild zeigte sich an lichtsignalgeregelten Knotenpunkten.

- Bei höheren Geschwindigkeiten von Radfahrern auf Streckenabschnitten stieg die Anzahl der Unfälle leicht an. Dieser Einfluss war jedoch durch die Radverkehrsstärke überlagert. In Straßen mit höheren Radfahrergeschwindigkeiten ereigneten sich vermehrt Unfälle im Längsverkehr sowie Unfälle durch ruhenden Verkehr.

In sechs Szenarien wurden anschließend mögliche Entwicklungstendenzen des Radverkehrs entwickelt und deren Auswirkungen auf die Verkehrssicherheit abgeschätzt.

S1: $\quad$ Kurzfristig moderate Zunahme des Radverkehrs

S2-A: $\quad$ Mittelfristige Veränderung der Altersstruktur

S2-A-20: Veränderung der Altersstruktur und moderate Zunahme des Radverkehrs

S2-A-20-V: Veränderung der Geschwindigkeiten aufgrund einer veränderten Altersstruktur und veränderter Fahrradtypen bei moderater Zunahme des Radverkehrs

S2-A-40: $\quad$ Veränderung der Altersstruktur und erheblich höhere Stärken des Radverkehrs

S2-A-40-V: Erheblich höhere Stärken und Veränderung der Geschwindigkeiten des Radverkehrs 
Die Szenarienberechnungen beruhten auf der heute üblichen Mischung teils anforderungsgerechter, teils aber auch mängelbehafteter Radverkehrsanlagen.

Sie zeigten, dass an Streckenabschnitten in Zukunft die Anzahl der Radverkehrsunfälle mit Personenschaden und noch stärker die Anzahl der Radverkehrsunfälle mit schwerem Personenschaden, vor allem bedingt durch den Anstieg der Radverkehrsstärken, stark zunehmen werden, wenn keine geeigneten Gegenmaßnahmen ergriffen werden. Unter den Bedingungen des Szenarios S2-A-40-V wird sich die Anzahl der Radverkehrsunfälle mit Personenschaden sogar mehr als verdoppeln und sich die Anzahl der schweren Radverkehrsunfälle im Vergleich zu den heutigen Zahlen um den Faktor 2,4 erhöhen. Bei Führung des Radverkehrs auf der Fahrbahn wie auch auf Radfahrstreifen ist mit Anstieg der Radverkehrsstärke die stärkste, auf Radwegen die geringste Zunahme an Radverkehrsunfällen zu verzeichnen.

An lichtsignalgeregelten Knotenpunkten konnte kein Anstieg der Unfallanzahl ermittelt werden, durch den demografischen Wandel können allerdings Unfälle mit schwerem Personenschaden auf einen Anteil von $16 \%$ an den Unfällen mit Personenschaden steigen.

Die Empfehlungen der Untersuchung beruhten auf den Erkenntnissen über den heutigen Radverkehr. Bereits heute bestehen Radverkehrsanlagen mit starkem Radverkehr bzw. mit höheren Geschwindigkeiten des Radverkehrs. Bereits heute sind alte Menschen im Radverkehr besonders gefährdet. Die Empfehlungen für den künftigen Radverkehr sind daher bereits heute relevant und sollten Anwendung finden, um einem Anstieg der Unfallzahlen entgegenzuwirken.

Grundsätzlich kommen in Zukunft an Verkehrsstraßen weiterhin alle bislang gebräuchlichen Führungsformen für den Radverkehr wie Radwege, Radfahrstreifen, Schutzstreifen und MischverkehrsFührungen in Betracht. Wegen ihrer niedrigen Unfallzahlen und geringer Zunahme der Unfallbelastung auch bei steigenden Radverkehrsstärken sollten Fahrradstraßen - soweit sich die Struktur des Straßennetzes dafür eignet, auch als Netzalternative zu Verkehrsstraßen - verstärkt Einsatz finden.

Zur Reduzierung der besonders zunehmenden Längsverkehrsunfälle sollten bei Mischverkehr (Unfälle mit überholenden $\mathrm{Kfz}$ ) Geschwindigkeitsbegrenzungen auf $30 \mathrm{~km} / \mathrm{h}$ geprüft werden. Bei Radwegen sind Breiten, die ein Überholen unter Radfahrern zulassen und damit Überholunfälle unter Radfahrern reduzieren, erforderlich. Bei Radfahrstreifen sind besonders ausreichende Sicherheitsräume zu KfzParkstreifen erforderlich.

Bei isolierter Betrachtung des demografischen Wandels (Szenario S2-A) sollten Streckenabschnitte und Knotenpunkte gleichermaßen auf die Sicherungsmaßnahmen hin betrachtet werden. Einem überproportionalen Anstieg von Abbiegen- und Einbiegen-/ Kreuzen-Unfällen müssen u. a. Sicherungsmaßnahmen an Einmündungen und Grundstückszufahrten begegnen. Hier besteht insbesondere bei Radwegen Handlungsbedarf.

Bei künftig erhöhten Geschwindigkeiten kann eine noch stärkere Zunahme von LängsverkehrsUnfällen und ein erhöhter Anteil von Unfällen durch ruhenden Verkehr erwartet werden. Dies unterstreicht zum einen die Notwendigkeit ausreichender Überholbreiten von Radwegen und Radfahrstreifen. Auch bei Mischverkehrsführungen sollten Sicherheitsräume zwischen der Fahrbahn und Parkstreifen geprüft werden. Radfahrergruppen, die - wie insbesondere jüngere Erwachsene oder Pedelec-Nutzer - mit höheren Geschwindigkeiten fahren, sollten verstärkt für besondere geschwindigkeitsbedingte Risiken sensibilisiert werden. 


\section{Abstract}

The demographic change, the increasing number of pedelecs and the higher share of cycle traffic in several cities lead to a substantial change of cycling traffic volume, traffic composition and cycling speeds. In the future, especially in cities, increasing cycle traffic volumes and diverse cycling speeds are expected. The intention of the study was to determine if these recent development influence the amount of cycling accidents and which measures should be taken to increase cycle safety.

For different forms of cycle facilities the study determined the influence of cycling traffic volume, cycling speeds and the age structure on the number, severity and types of accidents involving cyclists. The following main findings could be drawn from traffic counts, behavioral observations, speed measurements and accident analysis:

- Compared to previous studies, the average cycling speeds have increased by about $1.5 \mathrm{~km} / \mathrm{h}$ to about $18.2 \mathrm{~km} / \mathrm{h}$.

- With increasing bicycle traffic volumes the sections with minor intersecting streets show an increasing number of cycling accidents and a higher share of accidents with serious personal injuries. Similar findings could not be obtained at signalized intersections.

- Sections with a higher level of cycling speeds showed a shift to more accidents between vehicles moving along the same/ opposite way

- Special safety problems, especially at cycle paths, exist due to wrong-way driving

- Cyclists over 65 years have an about twice as high average accident risk compared to 25 to 65year-old cyclists. Similar findings could not be obtained at signalized intersections.

- Accidents involving older cyclists have a higher share of severe accidents at sections as well as at intersections

- Sections with higher cycling speeds showed a slightly increased number of accidents. However, this influence is superimposed by the bicycle traffic volume. Sections with higher cycling speeds showed a higher share of accidents between vehicles moving along the same/ opposite way and accidents involving stationary vehicles.

By developing six scenarios, possible future trends and their impact on cycle safety were estimated.

S1: $\quad$ moderate short term increase in bicycle traffic volume

S2-A: medium-term change in age structure

S2-A-20: $\quad$ change in age structure and a moderate increase of bicycle traffic volume

S2-A-20-V: moderate increase in bicycle traffic volume and a change in cycling speeds due to changes in age structure and new bicycle types

S2-A-40: change in age structure and significant increase of bicycle traffic volume

S2-A-40-V: $\quad$ significant increase of bicycle traffic volume and speeds.

The calculations for the scenarios were based on the actual mix of guideline conform and defective bicycle facilities.

The scenarios showed that the increasing number of cycle accidents with personal injuries at road sections, and even more the number of severe cycle accidents, is mainly due to the increase of bicycle traffic volume. Under the conditions of scenario S2-A-40-V, the number of bicycle accidents with personal injuries will be the double of today's level - severe bicycle accidents will even increase by a factor of 2.4. Concerning the several forms of bicycle facilities, the highest increase of accidents are expected on cycle lanes and on lanes with mixed traffic of cars and bicycles; the lowest increase in bicycle accidents are expected at cycle paths. Similar findings could not be obtained at signalized 
intersections. However, due to the changes in age structure the share of severe accidents may rise to a level of $16 \%$ of accidents with personal injuries.

The recommendations of the study are based on the knowledge of today's bicycle traffic. By now, there are already cycle facilities with high bicycle traffic volume or cycling speeds and elderly cyclists are already particularly at high risk. The recommendations for the future cyclists are therefore relevant today and should be applied to counteract the increase of cycle accidents.

Basically, common cycling facilities such as cycle paths, cycle lanes, lanes with mixed traffic and advisory cycle lanes are suitable facilities for the future. Because of their low average accident rate and their weak impact of bicycle traffic volume on cycle accidents, cycle tracks should - as far as infrastructural possible - be more used as an alternative to common cycling facilities.

To reduce the particularly increasing accidents between vehicles moving along the same/ opposite way, speed limits of $30 \mathrm{~km} / \mathrm{h}$ should be considered for lanes with mixed traffic. The widths of cycle paths should allow an overtaking among cyclists and thus reduce accidents among cyclists. At cycle lanes safety distances to parking cars are especially recommended.

Considering the isolated effect of changing age structure (scenario S2-A) the increase of accidents caused by crossing roads, turning into or off the roads need special consideration at sections and intersections. At cycle paths these types of accidents need to be considered at driveways as well.

In future, higher cycling speeds will increase accidents between vehicles moving along the same/ opposite way and accidents involving stationary vehicles even more. This emphasizes the need for sufficient widths of cycle paths and cycle lanes. Safety distances between traffic and parking cars should be considered at lanes with mixed traffic as well. Special groups of cyclists with higher average speeds - in particular young adults and users of pedelecs- should be sensitized to the specific speed-related risks. 


\section{Anlass der Untersuchung}

\section{$1.1 \quad$ Ausgangslage}

Aus verschiedenen Forschungsvorhaben und örtlichen Unfalluntersuchungen liegen Erkenntnisse über das Unfallrisiko, über typische Unfallabläufe und unfallbeeinflussende Verhaltensweisen von Radfahrern und Kraftfahrern als Unfallgegnern vor.

Durch den demografischen Wandel, die zunehmende Verbreitung von Pedelecs sowie die in mehreren Städten stark gestiegenen Anteile des Radverkehrs am Gesamtverkehr unterliegen die Stärken, Zusammensetzungen und Geschwindigkeiten des Radverkehrs derzeit einem erheblichen Wandel. Künftig werden - insbesondere im Stadtverkehr, aber auch zwischen den äußeren und inneren Zentrumsbereichen von Großstädten - aufgrund verschiedener Einflüsse zunehmende Radverkehrsstärken und stärker differenzierte Geschwindigkeiten von Radfahrern erwartet.

Über Auswirkungen dieser Veränderungen auf die Verkehrssicherheit und die hieraus erwachsenden Anforderungen an die Radverkehrsinfrastruktur an straßenverkehrsrechtliche Regelungen und an die Verkehrsaufklärung im Rahmen der Verkehrssicherheitsarbeit liegen bislang keine umfassenden und abgesicherten Erkenntnisse vor.

\section{$1.2 \quad$ Ziel}

Ziel der Untersuchung war deshalb zunächst die Verdichtung des Erkenntnisstandes über das Fahrverhalten, den Verkehrsablauf und die Unfallrisiken von Radfahrern

- bei unterschiedlichen Arten und baulich-betrieblichen Merkmalen von Radverkehrsanlagen,

- verschiedener soziodemografischer Gruppen und

- in Abhängigkeit des Verkehrsverhaltens und des Geschwindigkeitsniveaus bei höheren Radverkehrsstärken.

Diese Kriterien wurden vor dem Hintergrund der Anforderungen des zukünftigen Radverkehrs betrachtet. Hierzu wurden denkbare Szenarien entwickelt und der Fragestellung entsprechende Untersuchungsstrecken ausgewählt. Dazu zählten vor allem Abschnitte mit hohen Radverkehrsstärken, aber auch die Betrachtung einer zunehmenden Verbreitung von Pedelecs. Im Ergebnis wurden Aussagen abgeleitet zu künftigen Anforderungen

- an die Planung und den Entwurf von Radverkehrsanlagen,

- an straßenverkehrsrechtliche Regelungen und

- mit Blick auf die strategische Ausrichtung der Verkehrsaufklärung,

um den zukünftigen Entwicklungen besser Rechnung zu tragen. 


\section{Derzeitiger Erkenntnisstand}

Das folgende Kapitel stellt wesentliche Erkenntnisse über das Unfallgeschehen verschiedener Radfahrergruppen sowie bei unterschiedlichen baulichen Merkmalen von Radverkehrsanlagen in Deutschland zusammen. Weiterhin werden Erkenntnisse über die Geschwindigkeiten von Radfahrern dargestellt.

\subsection{Unfälle im Radverkehr}

In Deutschland verunglückten bei polizeilich erfassten Straßenverkehrsunfällen seit Mitte der 1990er Jahre bis 2004 in den meisten Jahren zwischen 70.000 und 75.000 Radfahrern. Seit 2005 stieg die Zahl der Verunglückten - mit Ausnahme des Jahres 2010 - auf jährlich etwa 76.000-79.000.

Die Zahl der getöteten Radfahrer war seit den 1990er Jahren von jährlich 600-900 auf jährlich etwa 400-450 gesunken. Die Zahl der schwerverletzten Radfahrer lag in den 1990er Jahren zwischen etwa 16.000 und 19.000. Seit dem Jahr 2000 dagegen lag sie fast durchgängig zwischen 14.000 und 15.000 Schwerverletzten je Jahr. Die Zahl der Leichtverletzten pro Jahr dagegen stieg seit den 90er Jahren von 50.000-60.000 auf zumeist über 60.000 (Bild 1, Bild 2) (Statistisches Bundesamt, versch. J.).

Die Zahl aller bei Straßenverkehrsunfällen getöteten oder schwerverletzten Verkehrsteilnehmer ist seit den 90er Jahren von jährlich etwa 130.000-140.000 auf etwa 70.000 gesunken. Der Radverkehr hat damit an dem allgemeinen Rückgang der Unfälle mit schwerem Personenschaden nicht teilgehabt.

Allerdings sind der Anteil des Radverkehrs an der Zahl alltäglicher Wege und die Verkehrsleistungen in diesem Zeitraum gestiegen. Nach der Studie „Mobilität in Deutschland“ stieg der Radverkehrsanteil von 9 \% im Jahr 2002 auf $10 \%$ im Jahr 2008 (BMVBS, 2004; BMVBS, 2010). 1991 betrug die Verkehrsleistung des Radverkehrs etwa 22 Mrd. Personen-km (Hautzinger, 1996), im Jahr 2002 30,9 Mrd. Pkm und im Jahr 200833 Mrd. Pkm (BMVBS, 2004; BMVBS, 2010)ํ․ Bezogen auf die Verkehrsleistung, ist das statistische Unfallrisiko von Radfahrern im Vergleich zu den frühen 1990er Jahren stark und im letzten Jahrzehnt noch leicht gesunken.

Zu berücksichtigen ist allerdings eine hohe Dunkelziffer polizeilich nicht erfasster Unfälle. Die Literatur benennt die Dunkelziffer mit 50-90 \% aller Radfahrerunfälle (Hautzinger, 1993), (Limbourg, 2008), (UDV, 2011b). Insbesondere Alleinunfälle ohne schwere Verletzungsfolge werden selten polizeilich erfasst (Schepers, 2014)

Etwa die Hälfte aller Radverkehrsunfälle ereignete sich an Knotenpunkten. Gleichzeitig bestimmten Vorfahrtunfälle das Unfallgeschehen, die vor allem an Knotenpunkten und Grundstückszufahrten im Allgemeinen im Zusammenhang mit unklaren Vorrangverhältnissen oder unzureichenden Sichtbeziehungen auftraten (Maier et al., 2010).

1 Die Radverkehrsanteile des Jahres 1991 einerseits und der Jahre 2002 und 2008 andererseits sind wegen geänderter Erhebungsmethoden nur eingeschränkt vergleichbar. Auch nach dem SrV sind die Verkehrsanteile und die Verkehrsleistung zwischen 2003 und 2008 gestiegen. 


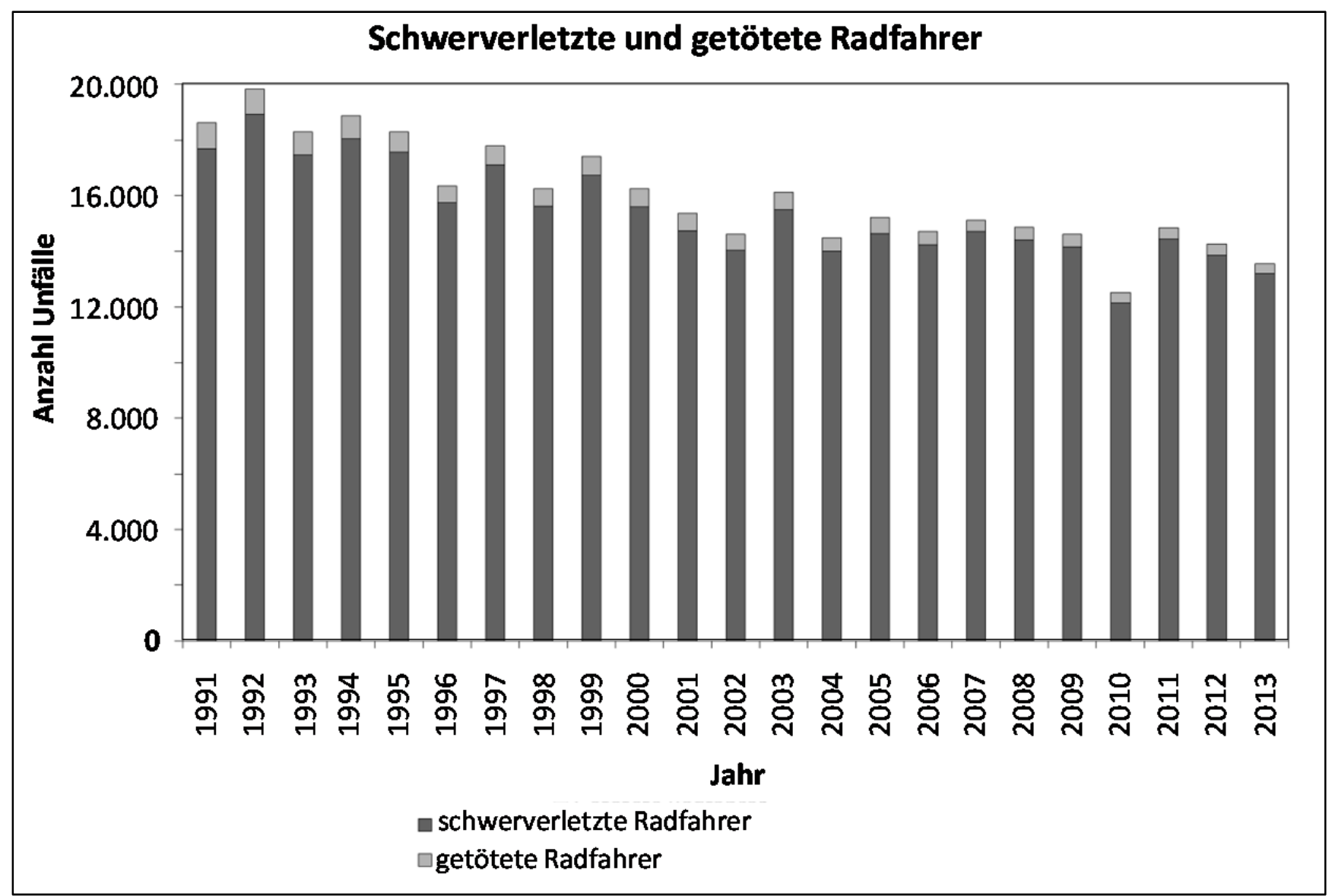

Bild 1: Entwicklung der Zahl schwer verletzter oder getöteter Radfahrer (Statistisches Bundesamt, versch. J.)

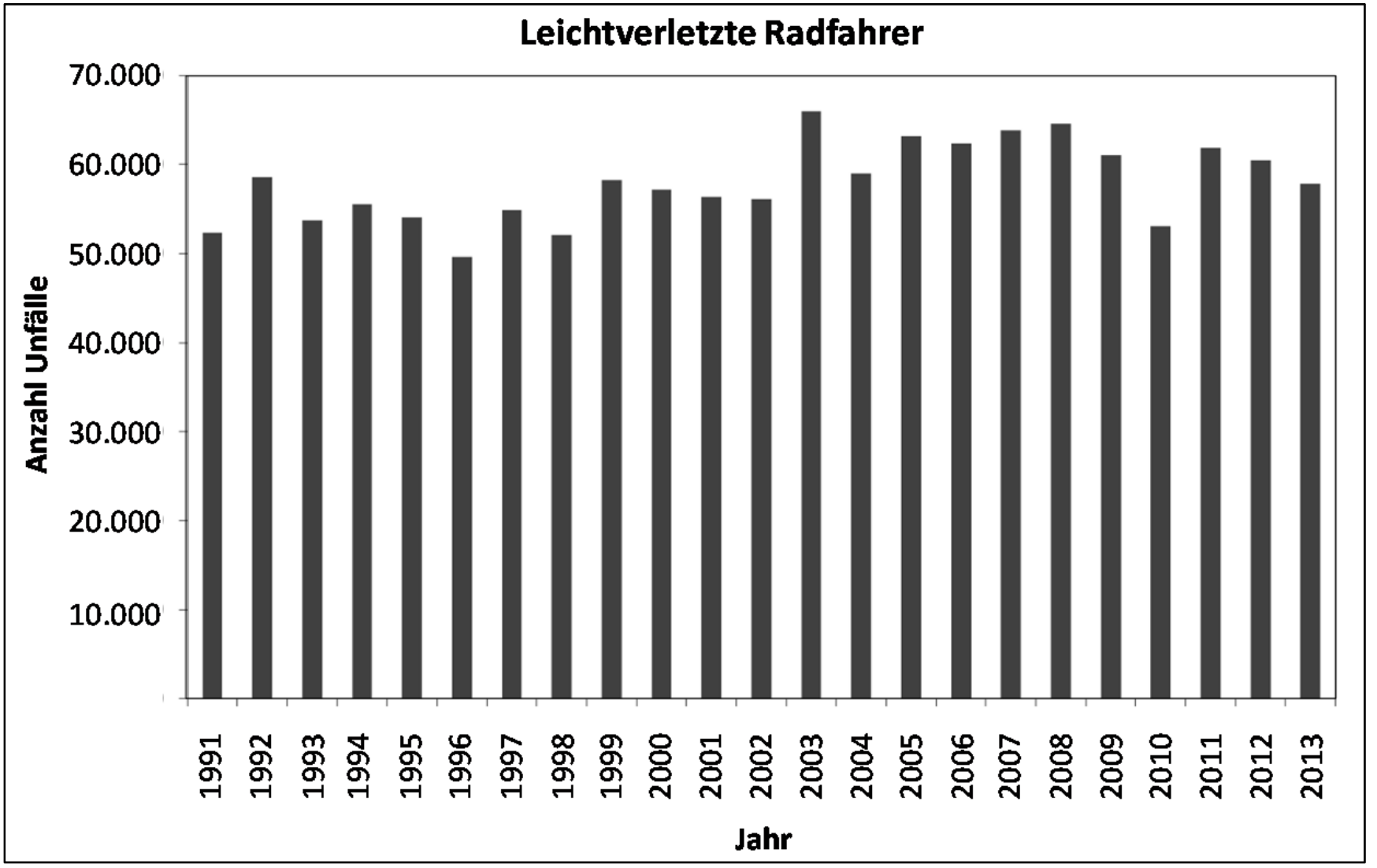




\subsection{Ausprägungen des Unfallgeschehens: besonders gefährdete Radfahrer-Gruppen}

\subsubsection{Alter und Geschlecht}

\section{Zahl verunglückter Radfahrer}

Im Jahr 2011 waren $14 \%$ der bei polizeilich erfassten Unfällen verunglückten Radfahrer Kinder bis 14 Jahre, etwa $16 \%$ waren 65 oder älter (Tabelle 1). Während die Zahl verunglückter Kinder und Jugendlicher in den letzten Jahren sank, stieg insbesondere die Zahl verunglückter Über-64-Jährige. In den Jahren 2004 und 2005 zum Beispiel waren etwa 18 \% der verunglückten Radfahrer Kinder bis 14 Jahren, etwa $13 \%$ waren 65 und älter (Statistisches Bundesamt, versch. J.).

Im Jahr 2012 waren 54 \% der innerorts getöteten Radfahrer 65 Jahre oder älter. Der Bevölkerungsanteil dieser Altersgruppe belief sich auf $22 \%{ }^{2}$

Insgesamt waren $61 \%$ der verunglückten Radfahrer männlich. Männer waren dabei in allen Altersgruppen deutlich häufiger vertreten als Frauen, die Unterschiede waren bei den jüngeren Jahrgängen besonders stark ausgeprägt.

Tabelle 1: Verunglückte Radfahrer nach Alter und Geschlecht 2011 (Statistisches Bundesamt, 2012)

\begin{tabular}{|c|c|c|c|c|c|c|}
\hline \multirow{3}{*}{ Altersgruppe } & \multicolumn{4}{|c|}{ Geschlecht } & \multirow{2}{*}{\multicolumn{2}{|c|}{ Gesamt }} \\
\hline & \multicolumn{2}{|r|}{ m } & \multicolumn{2}{|r|}{ w } & & \\
\hline & Anzahl & \begin{tabular}{|c|} 
Anteil an allen \\
verunglückten Radfahrern [\%]
\end{tabular} & Anzahl & $\begin{array}{c}\text { Anteil an allen } \\
\text { verunglückten Radfahrern [\%] }\end{array}$ & Anzahl & $\begin{array}{c}\text { Anteil an allen } \\
\text { verunglückten Radfahrern [\%] }\end{array}$ \\
\hline$<6$ & 344 & 0,4 & 161 & 0,2 & 505 & 0,7 \\
\hline $6-<10$ & 1.515 & 2,0 & 581 & 0,8 & 2.096 & 2,7 \\
\hline $10-<15$ & 5.347 & 7,0 & 2.970 & 3,9 & 8.317 & 10,8 \\
\hline $15-<18$ & 2.685 & 3,5 & 1.818 & 2,4 & 4.503 & 5,9 \\
\hline $18-<21$ & 1.787 & 2,3 & 1.350 & 1,8 & 3.137 & 4,1 \\
\hline $21-<25$ & 2.403 & 3,1 & 1.919 & 2,5 & 4.322 & 5,6 \\
\hline $25-<50$ & 16.433 & 21,4 & 9.567 & 12,5 & 26.000 & 33,9 \\
\hline $50-<65$ & 8.729 & 11,4 & 6.060 & 7,9 & 14.789 & 19,3 \\
\hline $65-<70$ & 2.109 & 2,8 & 1.548 & 2,0 & 3.657 & 4,8 \\
\hline $70-<75$ & 2.552 & 3,3 & 1.943 & 2,5 & 4.495 & 5,9 \\
\hline 75 und mehr & 2.724 & 3,6 & 1.927 & 2,5 & 4.651 & 6,1 \\
\hline k. A. & 134 & 0,2 & 49 & 0,1 & 183 & 0,2 \\
\hline Summe & 46.762 & 61,0 & 29.893 & 39,0 & 76.655 & 100,0 \\
\hline
\end{tabular}

2 Eigene Berechnungen auf Grundlagen der Daten des Statistischen Bundesamtes (Statistisches Bundesamt, versch. J., sowie Statistisches Bundesamt, 2014) 


\subsubsection{Verkehrsleistung und Unfallbeteiligung}

Bezogen auf die Verkehrsleistung im Radverkehr, verunglückten nach einer älteren Untersuchung insbesondere männliche Kinder und Jugendliche sowie Über-74-Jährige häufiger als Radfahrer anderer Altersgruppen. Bezogen auf die Verkehrsbeteiligungsdauer, verunglückten männliche Jugendliche im Alter von 10-14 Jahren, junge Männer zwischen 21 und 24 Jahren und Über-74-Jährige besonders häufig mit dem Fahrrad (Hautzinger, 1996).

Offen ist, inwieweit der Rückgang der Zahl verunglückter Kinder und Jugendlicher sowie der Anstieg bei Über-65-Jährige durch Veränderungen der Fahrradnutzung - wie etwa durch häufigere Fahrradfahrten oder höhere Verkehrsleistungen von Über-65-Jährigen im Radverkehr - beeinflusst wurde. Jüngere Untersuchungen über das verkehrsleistungsbezogene Unfallrisiko verschiedener Altersgruppen waren nicht ersichtlich.

Auf Grundlage der bei den Trägern der gesetzlichen Unfallversicherungen vorliegenden Unfalldaten sowie von Mobilitätskennziffern des Jahres 2002 (BMVBS, 2003; BMVBS, 2004) wurde das Unfallgeschehen im Wirtschaftsverkehr ermittelt (Geiler, 2007). Das Verletzungsrisiko von Frauen und Männern beim Radfahren im Wirtschaftsverkehr war etwa gleich hoch. Bezogen auf das Alter ergab sich für die 18-bis 29-Jährigen das höchste Verletzungsrisiko (Tabelle 2).

Tabelle 2: Verletzungsrisiko nach Altersgruppen bei Radfahrern im Wirtschaftsverkehr (Geiler, 2007)

\begin{tabular}{|l|c|c|}
\hline \multicolumn{1}{c|}{$\begin{array}{c}\text { Alter } \\
\text { [Jahre] }\end{array}$} & $\begin{array}{c}\text { Verletzungsrate } \\
\text { [Verletzte pro 1 Mio. Pkm] }\end{array}$ & $\begin{array}{c}\text { Verletzungszeitrate } \\
\text { [Verletzte pro } \\
\text { 1 Mio. Verkehrsbeteiligungsstunden] }\end{array}$ \\
\hline $\mathbf{0 - 1 7}$ & 4,96 & 25,99 \\
\hline $\mathbf{1 8 - 2 9}$ & 7,67 & 74,51 \\
\hline $\mathbf{3 0 - 4 4}$ & 4,70 & 56,65 \\
\hline $\mathbf{4 5 - 5 9}$ & 5,18 & 56,88 \\
\hline$>\mathbf{6 0}$ & 5,70 & 18,27 \\
\hline
\end{tabular}

\subsubsection{Verhaltensbezogene Einflüsse}

Das erhöhte Unfallrisiko männlicher Jugendlicher wurde vor allem mit einem in der Pubertät ausgeprägten Risikoverhalten begründet (Limbourg, 2008; Funk, 2010). Die hohe Unfallbeteiligung von Über-65-Jährige wurde teils auf mit zunehmendem Alter nachlassende Seh- und Hörvermögen sowie kognitive und motorische Fähigkeiten zurückgeführt. So verunglückten Über-65-Jährige im Vergleich zu anderen Radfahrern anderer Altersgruppen häufiger an Vorfahrtgeregelten Knoten oder beim Abbiegen (Boenke, 2011). Gegen die Annahme eines Einflusses nachlassender Fähigkeiten zur Verkehrsteilnahme auf die Unfallbeteiligung könnte jedoch sprechen, dass Über-65-Jährige, die mit „konventionellen“ Fahrrädern am Verkehr teilnahmen, sich ihrer altersbedingten Einschränkungen bewusst waren und diese durch das Verkehrsverhalten kompensierten (Steffens, 1999).

Nach einer Untersuchung in Münster unter Einbeziehung von Krankenhausdaten stellten - im Vergleich zur Altersverteilung der Gesamtbevölkerung - 20-bis 29-Jährige einen hohen Anteil an Unfallopfern im Radverkehr. Für diese Altersgruppe mit vielen Studierenden ist das Fahrrad allerdings auch das am häufigsten benutzte Verkehrsmittel. Zu berücksichtigen ist ein altersspezifisches Freizeitverhalten mit häufigen Partys und die nach Datenlage der Polizei häufige alkoholbeeinflusste Fahrradnutzung (UDV, 2011b). 


\subsubsection{Tragen eines Helms}

Für Deutschland lag eine medizinische und unfalltechnische Untersuchung über die Unfallverläufe und die Verletzungsarten von etwa 3.250 verunglückten Radfahrern ohne Schutzhelm und etwa 270 Radfahrern mit Schutzhelm vor (Unfalljahre 1999-2007, Unfallorte in den Großräumen Dresden und Hannover). Unter den verunglückten Radfahrern waren etwa 500 Bis-15-Jährige und fast 3.000 Über15-Jährige. Hiernach erfuhren Radhelmbenutzer gegenüber Nichthelmträgern statistisch signifikant

- seltener Kopfverletzungen (mit Helm 72 \% unverletzt, ohne Helm 61 \%),

- um $33 \%$ seltener schwere Kopfverletzungen,

- um $15 \%$ weniger isolierte Weichteilverletzungen und

- um $23 \%$ seltener Schädelfrakturen.

Tendenziell erlitten Helmträger auch seltener schwere Hirn- sowie Gesichtsverletzungen. (Otte, 2008) $)^{3}$

\subsubsection{Nutzung von Elektrofahrrädern (Pedelecs 25 und Pedelecs 45)}

Besondere Unfallrisiken wurden für die Nutzung von Pedelecs 45 diskutiert.

Die Unfallforschung der Versicherer führte - mit einem Schwerpunkt auf bis zu $45 \mathrm{~km} / \mathrm{h}$ motorisch unterstützten Pedelecs 45 - umfangreiche Fahrversuche und Crashtests durch. Besondere Unfallrisiken können durch folgende Umstände entstehen (UDV, 2011a):

- Beim Anfahren konnten deutliche Instabilitäten auftreten.

- Bei einem seitlichen Anprall auf Pkw bestand für Pedelec 45-Nutzer das Risiko schwerer Kopfverletzungen. Die UDV stufte das Risiko dieses für Grundstücksausfahrten und Knotenpunkte charakteristischen Unfalltyps als sehr hoch ein, da die Geschwindigkeit von Pedelec-Nutzern für Autofahrer schwerer einzuschätzen sei.

- Bei Überholvorgängen könnten auf Radverkehrsanlagen mit in der Praxis noch oft üblichen geringen Breiten Pedelec 25-Nutzer auf Radfahrer in Längsrichtung auffahren und für beide Beteiligte erhebliche Verletzungen auftreten.

- Bei bestimmten Bremsbauarten verzögerte sich der Bremsvorgang bis zum Stillstand bauartbedingt erheblich (so auch (Stiftung Warentest, 2011)).

Daten über die Unfallbeteiligung von Pedelecs 45 waren zum Zeitpunkt der Auswertung nicht bundesweit verfügbar. Es wurden jedoch Grundlagen für eine gesonderte polizeiliche Erfassung der Elektrozweiradverkehrsunfälle geschaffen. Seit Januar 2014 sind die Verkehrsbeteiligungsarten „Pedelec" und „E-Bike“ verpflichtend für die Statistik zu verwenden. ${ }^{4}$

Aussagen über das Unfallrisiko von Trägern und Nicht-Trägern eines Helms ließ diese Untersuchung nicht zu. Es wurde diskutiert, inwieweit überholende Kfz-Fahrer zu Radfahrern mit Helm einen geringeren Seitenabstand einhalten. Aus stichprobenhaften Untersuchungen lagen hierzu keine belastbaren Erkenntnisse vor.

$4 \quad$ Für die vorliegende Untersuchung wurden im Folgenden vorrangig Pedelecs 25 betrachtet, die straßenverkehrsrechtlich als Fahrräder eingestuft sind. In der Beteiligungsart „E-Bike“ sind sowohl Pedelec 45 mit Tretunterstützung als auch E-Bikes mit elektromotorischem Antrieb enthalten. 
Eine Sonderrecherche und Analyse von 1.118 polizeilich erfassten Pedelec 25 und -45-Unfällen in Baden-Württemberg kommt zu dem Schluss, dass „im Vergleich zum herkömmlichen Fahrrad keine höhere Unfallbeteiligung von Elektrofahrrädern (besteht). Es konnte aber bestätigt werden, dass die Unfälle mit E-Fahrrädern schlimmere Folgen haben, weil es dabei verhältnismäßig mehr Getötete und Verletzte gab. Der Anteil der Getöteten und Verletzten lag bei den Elektrozweiradfahrern bei 91,6 Prozent, bei den normalen Fahrradunfällen bei 86,8 Prozent. Davon waren 0,5 Prozent getötete Radfahrer, aber 1,6 Prozent Elektrozweiradfahrer. Ursächlich hierfür könnten die höheren Geschwindigkeiten der Elektrofahrräder sein." (Lawinger, 2012).

Als unfallbeeinflussende Faktoren wurden hier benannt (Angaben Unfallbeteiligter Nutzer von Pedelecs 25 und -45):

- höhere Geschwindigkeiten mit längeren, oft als viel zu kurz eingeschätzten Anhaltewegen

- Unterschätzung der Geschwindigkeiten von Elektrofahrrädern durch andere Verkehrsteilnehmer in Zusammenhang mit einem Unfallschwerpunkt an Ausfahrten und Kreuzungen

- „Geräuschlosigkeit“ insbesondere bei E-Rollern in Kombination mit der meist ausgenützten Höchstgeschwindigkeit von $45 \mathrm{~km} / \mathrm{h}$

Zwischen Pedelecs 25 und Pedelecs 45 zeigten sich - auf schmaler Datenbasis mit einer geringen Anzahl unfallbeteiligter Pedelecs 45 - keine belastbaren Unterschiede in der Unfallbeteiligung (Lawinger, 2012).

In einem aktuellen Forschungsprojekt der Unfallforschung der Versicherer konnte festgestellt werden, dass Pedelec 25-Fahrer mit einer etwa 1-2 km/h höheren Durchschnittsgeschwindigkeit als Fahrradfahrer fahren. Darüber hinaus konnten im Konfliktgeschehen zwischen Pedelec 25-Fahrern und Fahrradfahrern keine wesentlichen Unterschiede festgestellt werden. Wenn auch auf einer geringen Fallzahl basierend, zeigten sich jedoch für Pedelec 45-Fahrer $(n=10)$ deutlich höhere Durchschnittsgeschwindigkeiten $(+8 \mathrm{~km} / \mathrm{h})$, allerdings ohne dabei in eine höhere Anzahl kritischer Situationen involviert zu sein (UDV, 2014). 


\subsection{Unfallgeschehen auf Streckenabschnitten mit nicht lichtsignalge- regelten Anschlussknoten}

\subsubsection{Radverkehrsführung auf Radwegen, Radfahrstreifen und Schutzstreifen}

In einem Forschungsvorhaben der Bundesanstalt für Straßenwesen wurde das Unfallgeschehen auf Verkehrsstraßen mit benutzungspflichtigen Radwegen, nicht benutzungspflichtigen Radwegen sowie mit Radfahrstreifen oder Schutzstreifen untersucht (Alrutz, 2009). Wesentliche Ergebnisse für die Streckenabschnitte (einschließlich Einmündungen, ohne lichtsignalgeregelte Verkehrsstraßenknotenpunkte) waren:

- Bei Straßen mit benutzungspflichtigen oder auch nicht benutzungspflichtigen Radwegen stieg die Unfalldichte mit den Radverkehrsstärken. Bei Radfahr- und Schutzstreifen wurde kein belastbarer Zusammenhang ermittelt.

- Bei jedem Anlagentyp streuten die Unfallraten des Radverkehrs in einem weiten Bereich. Insgesamt prägten vor allem baulich-betriebliche Einzelmerkmale das Unfallgeschehen.

- Unfälle an Anschlussknoten zwischen in rechter Fahrtrichtung geradeaus fahrenden und rechts abbiegenden $\mathrm{Kfz}$ nahmen bei jedem der vier Anlagentypen gegenüber anderen Unfallverläufen einen herausgehobenen Anteil ein. Ebenfalls häufige Unfälle mit parkenden $\mathrm{Kfz}$ gingen auf Straßen mit nicht benutzungspflichtigen Radwegen weitaus überwiegend auf öffnende Beifahrertüren parkender Kfz zurück. Unfälle mit öffnenden Fahrertüren, die auch mit einer Fahrbahnnutzung der Radfahrer in Verbindung stehen können, traten bei Radwegen kaum auf. Auf Straßen mit Radfahrstreifen, insbesondere aber auf denen mit Schutzstreifen waren Unfälle mit öffnenden Fahrertüren stärker ausgeprägt. Längsverkehrs-Unfälle mit Kfz traten bei den Radfahrstreifen bzw. den Schutzstreifen insgesamt sehr selten, bei den Radwegen in Einzelfällen unter Beteiligung auf der Fahrbahn fahrender Radfahrer auf.

- Herausragende Anteile hatten insgesamt Unfälle mit Beteiligung regelwidrig links fahrender Radfahrer. Die mittlere Unfallrate der links Fahrenden lag bei den Straßen mit Radwegen in 4-6-facher Höhe der Unfallrate Rechtsfahrender, bei den Straßen mit Radfahrstreifen oder Schutzstreifen in 7bzw. 10-facher Höhe.

- Die mittlere Unfallrate rechts fahrender Radfahrer war bei nach den technischen Regelwerken anforderungsgerechten Radwegen niedriger als bei nicht anforderungsgerechten Radwegen. Die Unfallbelastung anforderungsgerechter Radfahrstreifen war annähernd mit der bei anforderungsgerechten Radwegen vergleichbar.

- Bei jedem Anlagentyp kam ausreichenden Sichtbeziehungen zwischen der Radverkehrsanlage und der parallelen Kfz-Fahrstreifen sowie zu einmündenden Fahrbahnen bzw. Grundstückszufahrten hohe Bedeutung für die Sicherheit zu. Fast alle Unfälle durch ruhenden Verkehr ereigneten sich auf Straßen mit schmalen Sicherheitsräumen zwischen der Radverkehrsanlage und Kfz-Parkständen. Bei den untersuchten Radfahrstreifen und Schutzstreifen war ein Einfluss der Kfz-Verkehrsstärke auf die Unfallbelastung des Radverkehrs nicht nachweisbar.

- An Anschlussknotenpunkten ohne Lichtsignalanlage begünstigten Radschutzstreifen und Radfahrstreifen Unfälle mit linksabbiegenden $\mathrm{Kfz}$, die Lücken zwischen verkehrsbedingt haltenden Fahrzeugen (Stau) beim Abbiegen nutzten (Goth, 2005).

So wiesen Streckenabschnitte mit Radwegen, die bei hohen Radverkehrsstärken zu schmal dimensioniert waren und teilweise auch die Anforderungen der VwV-StVO (2009) an die für eine Anordnung der Benutzungspflicht erforderliche Breite unterschritten, eine erhebliche Anzahl von Unfällen beim Überholen von Radfahrern untereinander auf (Ortlepp, 2008), ähnlich (Angenendt, 1993) und (Alrutz, 2008). 
Die Unfallquote an Einmündungen von Erschließungsstraßen konnte durch Teilaufpflasterung der Einmündungsbereiche gegenüber markierten Radfahrerfurten deutlich reduziert werden. Dies galt für das Rechtseinbiegen sowie Rechts- und Linksabbiegen der Kfz. Auch Roteinfärbungen wiesen in der Tendenz eine günstigere Sicherheitsbilanz auf (Schnüll, 1992).

\subsubsection{Radverkehrsführung im Mischverkehr}

Nach einer Untersuchung aus dem Jahr 1993 wiesen Fahrbahnführungen des Radverkehrs vergleichbar hohe Unfallraten wie Radwegführungen auf. In dem Verkehrsstärkenbereich von 5.000-25.000 Kfz/Tag stieg die Unfallrate des Radverkehrs tendenziell mit der Kfz-Verkehrsstärke. Etwa zwei Drittel der Radfahrerunfälle im Mischverkehr ereigneten sich auf Streckenabschnitten (ohne Einmündungen), etwa ein Drittel an Einmündungen. Auf Streckenabschnitten standen Alleinunfälle, Unfälle durch ruhenden Verkehr (Kollision mit einer öffnenden Fahrertür) und Unfälle im Längsverkehr zwischen Radfahrern und überholenden Kfz im Vordergrund. 49 \% der Knotenunfälle waren Einbiegen-/ Kreuzen-Unfälle. An nicht lichtsignalgeregelten Knotenpunkten waren bei Führung im Mischverkehr $29 \%$ der Unfälle Abbiegen-Unfälle, überwiegend mit links abbiegenden Kfz (Angenendt, 1993). Nach örtlichen Untersuchungen geschahen in Abschnitten mit engen Straßenraumbreiten, hohem Parkdruck und häufigen Parkwechselvorgängen viele Unfälle auch durch das unachtsame Öffnen der Fahrertür (Alrutz, 2008).

Nach einer gezielten Untersuchung von Abbiege-Unfällen waren nicht lichtsignalgeregelte Knotenpunkte mit Fahrbahnführung des Radverkehrs unfallauffällig in Bezug auf Linksabbiegeunfälle. Diese Unfälle ereigneten sich insbesondere, wenn besonders selten mit Radfahrern zu rechnen war oder selten links abgebogen wurde. Auf einigen Fallbeispielen dieser Untersuchung bestand jedoch ein Einfluss durch eine „Gehweg/Radfahrer frei“-Lösung (Kolrep-Rometsch et al., 2013).

\subsubsection{Fahrradstraßen}

Zu Fahrradstraßen ist der Kenntnisstand über das Unfallgeschehen eher gering. Erfahrungen lagen lediglich aus der Untersuchung einzelner Beispiele vor. Radfahrer-Unfälle mit Personenschaden stellten hiernach singuläre Ereignisse dar (Alrutz et al., 2001; Ruwenstroth et al., 1991). Ein laufendes UDV-Projekt untersucht derzeit Fahrradstraßen auf breiterer Basis.

\subsection{Radfahrer-Unfälle an Knotenpunkten mit Lichtsignalanlage}

Radfahrer-Unfälle an lichtsignalgeregelten Knotenpunkten haben einen hohen Anteil an allen Radfahrer-Unfällen (Ortlepp, 2008). Durch die zeitliche Separation potentieller Konfliktströme des Rad- und des Kfz-Verkehrs konnte eine günstige Bilanz im Unfallgeschehen des Radverkehrs erreicht werden. Eine gleichzeitige Freigabe bedingt verträglicher Verkehrsströme geradeausfahrender Radfahrer und abbiegender $\mathrm{Kfz}$ bedingte insbesondere bei hohen Rad- und Kfz-Verkehrsstärken besondere Unfallrisiken (Hass-Klau et al., 1992). Eine jüngere Untersuchung der UDV empfahl eine vollständig signaltechnisch gesicherte Führung des Radverkehrs gegenüber Rechts- wie auch gegenüber Linksabbieger-Kfz vor allem bei:

- hohen Abbiegeverkehrsstärken

- zügiger Linienführung der Abbieger (große Radien)

- hohen Geschwindigkeiten des Radverkehrs (z. B. Gefällestrecken)

- komplexen oder unübersichtlichen Knotenpunkten sowie

- Sichthindernissen, die nicht beseitigt werden können (Kolrep-Rometsch et al., 2013).

Sofern keine separaten Phasen vorgesehen sind, verbleibt der Konflikt zwischen geradeausfahrenden Radfahrern und abbiegenden Kfz. Dem Konflikt mit linksabbiegenden Kfz muss dabei ebenso große Bedeutung beigemessen werden wie dem mit rechtsabbiegenden Kfz (Hass-Klau et al., 1992). 
Weitere Unfälle ereigneten sich häufig zwischen bei Rot querenden Radfahrern und kreuzenden Kfz (Ortlepp, 2008).

Die „Unfallquote“5 für geradeausfahrende Radfahrer an lichtsignalgeregelten Knotenpunkten war nach einer älteren Untersuchung bei Führung auf der Fahrbahn oder auf Radfahrstreifen niedriger als bei Führungen auf Radwegen. Bei Fahrbahn- und Radfahrstreifenführungen war die geringere Zahl regelwidrig linksfahrender Radfahrer neben der geringen Verletzungswahrscheinlichkeit der Radfahrer durch rechts- und linksabbiegende Kfz gegenüber Radwegfurten ein Vorteil (Schnüll, 1992). Nach einer UDV-Untersuchung zu Abbiegeunfällen wiesen lichtsignalgeregelte Knotenpunktarme mit Radfahr- oder Schutzstreifenführung in Bezug auf Rechts- wie auf Linksabbiegeunfälle demgegenüber hohe Unfallquoten, allerdings auch niedrige Unfallraten auf. Die verhältnismäßig hohen Unfallquoten wurden durch die beobachteten hohen Verkehrsstärken der Radfahrer und abbiegenden Kfz stark relativiert. Das individuelle Unfallrisiko für geradeausfahrende Radfahrer und abbiegende Kfz war auf Radfahr- oder Schutzstreifen demnach eher gering (Kolrep-Rometsch et al., 2013).

Das Linksabbiegen von Kraftfahrzeugen war in der Unfallbilanz sowohl bei der Führung des Radverkehrs auf dem Radweg als auch auf der Fahrbahn oder auf Radfahrstreifen von großer Bedeutung. Der hohe Zeitdruck, unter dem linksabbiegende Kraftfahrzeuge häufig Zeitlücken im Gegenverkehrsstrom nutzen mussten (sofern keine Sonderphasen oder Zugabezeiten geschaltet sind), führte hier unabhängig von der Art der Radverkehrsführung zu einer Gefährdung entgegenkommender Radfahrer. Maßnahmen der Lichtsignalsteuerung wie Sonderphasen für Linksabbieger oder Zugabezeiten am Grünzeitende dienten auch im Wesentlichen dem Schutz von Radfahrern vor linksabbiegenden Kraftfahrzeugen (Hass-Klau et al, 1992). Gegenüber Knotenpunkten ohne Lichtsignalanlage wiesen Unfälle zwischen linksabbiegenden $\mathrm{Kfz}$ und Radfahrern an lichtsignalgeregelten Knotenpunkten eine höhere Unfallschwere auf (Goth, 2005).

Lichtsignalgeregelte Knotenarme mit Radwegführungen und weiter Absetzung der Radwegfurt (über $4 \mathrm{~m}$ Absetzung) wiesen nach einer Untersuchung aus dem Jahr 1992 höhere Unfallquoten als geringe oder mittlere Absetzungen der Furt auf. Die höheren Unfallquoten bei weiter Absetzung von Radwegfurten waren hiernach durch

- Radfahrer-Radfahrer-Unfälle im Längsverkehr sowie Alleinunfälle von Radfahrern, gerade im Zusammenhang mit starken Verschwenkungen der Radwege, sowie

- häufige Unfälle zwischen in rechter Richtung geradeausfahrenden Radfahrern und links abbiegenden $\mathrm{Kfz}$

geprägt (Schnüll, 1992). Nach der o. g. Untersuchung der UDV wiesen lichtsignalgeregelte Knotenarme mit mittlerer und weiter Absetzung der Radwegfurt (> $2 \mathrm{~m}$ ) in Bezug auf Rechtsabbiegeunfälle demgegenüber hohe „Unfallquoten“ und hohe Unfallraten auf. Als wesentlicher Einflussfaktor auf die Unfallzahlen wurden dabei lokale Sichthindernisse in den Knotenpunktzufahrten genannt. (KolrepRometsch et al., 2013).

An großen Knotenpunkten kam der Behandlung linksabbiegender Radfahrer besondere Bedeutung zu (Hass-Klau et al., 1992): 
- Ein vor dem Aufstellbereich des Kfz-Verkehrs liegender aufgeweiteter Aufstellbereich für Radfahrer erwies sich als unter Sicherheits- und Akzeptanzaspekten gut funktionierende Lösung, die nur relativ geringen Aufwand erfordert. Die Lösung ist bei beengten Platzverhältnissen und starkem Radverkehr geeignet. Ihre Einsatzbereiche liegen vor allem bei den insgesamt schwächer belasteten Zufahrten lichtsignalgeregelter Knotenpunkte.

- Dagegen können Radfahrerschleusen auch in hochbelasteten Hauptzufahrten der Knotenpunkte zum Einsatz kommen, da sie den abbiegenden Radfahrern vollen Signalschutz gewähren. Bei richtiger Steuerung wird die Leistungsfähigkeit für den Kfz-Verkehr nicht gemindert. Für Radfahrer entstehen längere Wartezeiten, die bei einem Teil der Radfahrer zur Nichtakzeptanz führt.

An freien Rechtsabbiegefahrbahnen traten Unfälle zwischen rechtsabbiegenden Kfz und geradeausfahrenden Radfahrern erheblich häufiger als bei Einbeziehung der rechtsabbiegenden Kfz in die Signalisierung auf (Schnüll, 1992; Ortlepp, 2008). 


\subsection{Geschwindigkeiten des Radverkehrs und geschwindigkeitsbeding- te Verletzungsschwere}

\subsubsection{Geschwindigkeiten des Radverkehrs}

Nach Schopf (1992) umfasste das Spektrum der Geschwindigkeiten im Radverkehr mit Fahrrädern ohne elektromotorische Unterstützung den Bereich zwischen 12,6 km/h und 25,2 km/h. Die Durchschnittsgeschwindigkeit wurde mit ca. $16 \mathrm{~km} / \mathrm{h}$ angegeben. Gleichzeitig wurden sowohl das Alter des Radfahrers, als auch der Wegezweck als signifikante Einflussgrößen der gefahrenen Geschwindigkeiten beschrieben. So fuhren nach Schopf insbesondere junge Radfahrer schneller. Die Durchschnittswerte der Altersgruppen bis 45 Jahre lagen über dem Mittelwert aller Radfahrer (Tabelle 3$)^{6}$. Außerdem fuhren Radfahrer im Freizeitverkehr langsamer als solche im Berufsverkehr (Bild 3) ${ }^{7}$.

Tabelle 3: Durchschnittsgeschwindigkeiten von Radfahrern nach Altersgruppen (Schopf, 1992)

\begin{tabular}{|l|c|c|}
\hline $\begin{array}{c}\text { Altersgruppe } \\
\text { [Jahre] }\end{array}$ & $\begin{array}{c}\text { Durchschnittsgeschwindigkeiten } \\
{[\mathbf{k m} / \mathbf{h}]}\end{array}$ & $\mathbf{N}$ \\
\hline unter $\mathbf{1 2}$ & 18,7 & 30 \\
\hline $\mathbf{1 3 - 1 8}$ & 20,5 & 60 \\
\hline $\mathbf{1 9 - 4 5}$ & 17,1 & 160 \\
\hline $\mathbf{4 6 - 6 0}$ & 13,7 & 30 \\
\hline über $\mathbf{6 0}$ & 12,6 & 20 \\
\hline
\end{tabular}

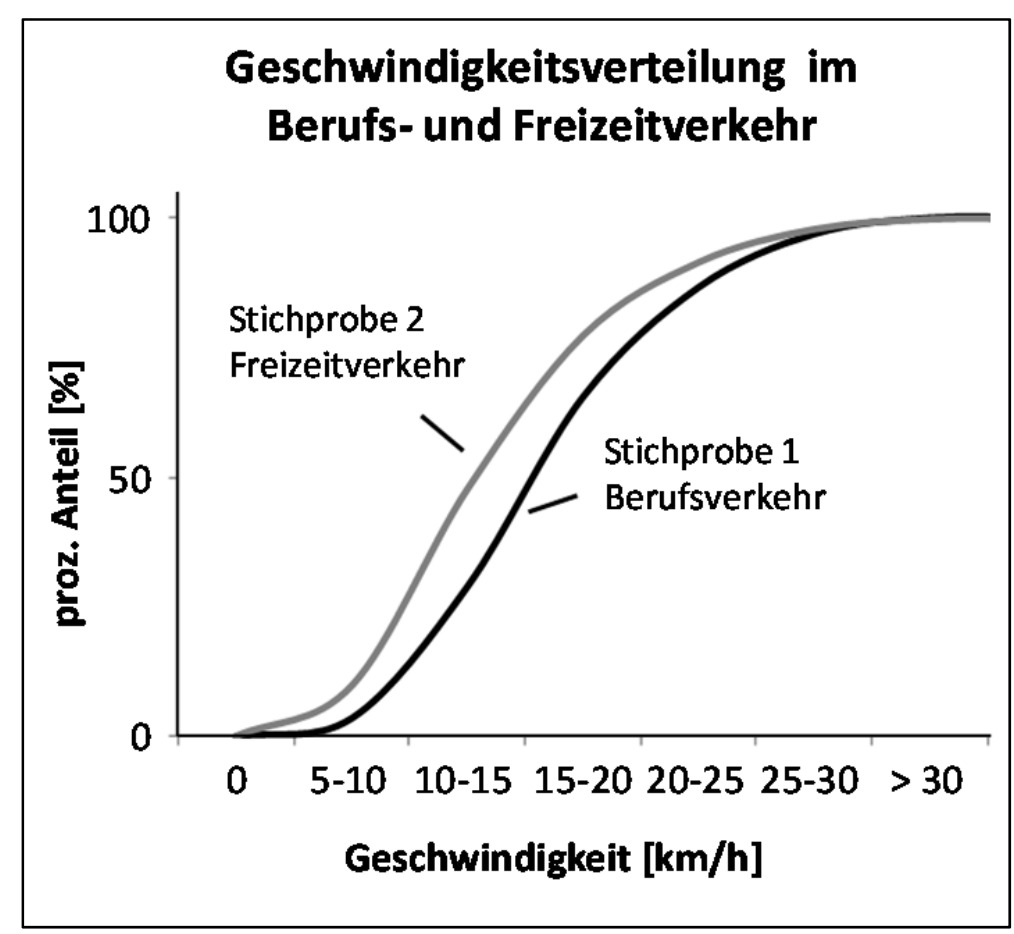

Bild 3: Geschwindigkeitsverteilung der Radfahrer im Berufs- und Freizeitverkehr ( $N=150$ je Stichprobe, Altersverteilung jeweils vergleichbar mit Tabelle 3) (Schopf, 1992)

6 Fahrtgeschwindigkeit auf $40 \mathrm{~m}$ Beobachtungslänge, Aufnahme durch Spiegelreflexkamera mit Motor.

7 Methode: Videobeobachtung. Zu Altersgruppen und Fahrradtyp k. A. 
Falkenberg et al. ermittelten für etwa 6.400 Radfahrer durchschnittliche Geschwindigkeiten von $16,9 \mathrm{~km} / \mathrm{h}$ bei freier und $16,8 \mathrm{~km} / \mathrm{h}$ bei beeinflusster Fahrt. Ein Drittel aller Radfahrer bewegte sich in einem mittleren Geschwindigkeitsbereich von 16-19 km/h, ein Viertel bzw. ein Sechstel fuhren mit 1316 bzw. 10-21 km/h. Geschwindigkeiten über 27 km/h traten selten auf (Bild 4).

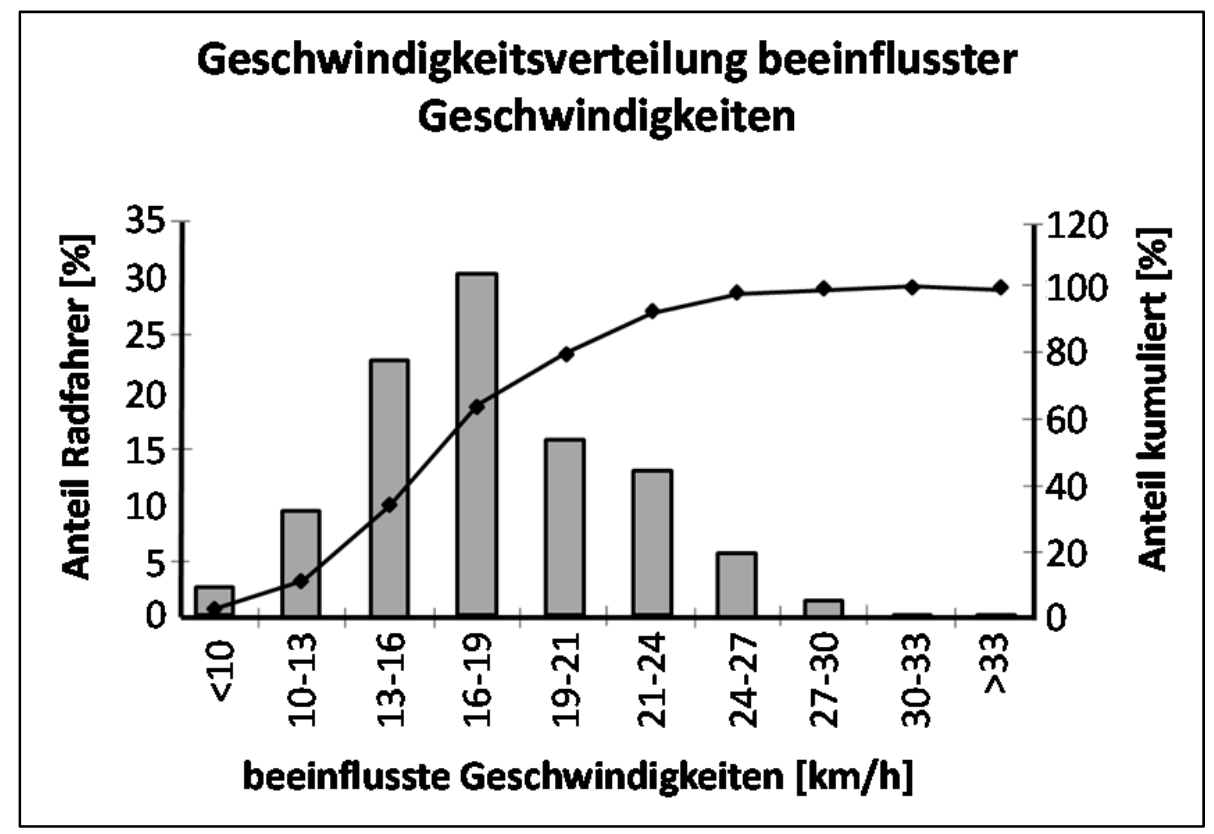

Bild 4: Häufigkeiten der beeinflussten Geschwindigkeiten (Falkenberg et al., 2003)

\subsubsection{Kollisionsgeschwindigkeit und Verletzungsschwere}

Die Analyse der die Unfall- bzw. Verletzungsschwere beeinflussenden Faktoren war Gegenstand verschiedenerer Analysen der GIDAS ${ }^{8}$-Unfalldatenbank (Hannawald, 2008; Richter, 2011). Die in der Regel mit multikriteriellen Methoden ausgewerteten Daten zeigten immer wieder einen engen Zusammenhang zwischen der Geschwindigkeit und der Schwere von Unfällen.

Richter (2011) entwickelte Verletzungsrisikofunktionen u. a. für Radfahrer, welche infolge eines Zusammenstoßes mit einem Pkw verletzt wurden. Als Zielgröße diente die Schwerebewertung nach der medizinischen Bewertungsskala MAIS ${ }^{9}$. Aufbauend auf einer univariaten Betrachtung wurden die Daten mit Hilfe von logistischer Regression auch einer multivariaten Analyse unterzogen. Als signifikant stellten sich dabei folgende Variablen heraus:

8 Die German In-Depth Accident Study (GIDAS) ist ein Projekt der Bundesanstalt für Straßenwesen (BASt) und der Forschungsvereinigung Automobiltechnik e. V. (FAT). Sie dokumentiert in zwei Untersuchungsgebieten in Deutschland stichprobenartig Verkehrsunfälle mit Personenschaden.

Maximum Abbreviated Injury Scale (MAIS). Die AIS bewertet die Schwere von Einzelverletzungen auf Basis der Überlebenswahrscheinlichkeit. 
- Alter: Die Gruppe der Ab-60-Jährigen wies im Vergleich der bis-20-Jährigen einen höheren Anteil schwerverletzter (insb. MAIS 3+) Personen auf.

- Ortslage: Das Risiko (Odds Ratio) außerhalb einer Ortsgrenze schwer verletzt zu werden, war um das 2,3-fache erhöht.

- Anprall am Fahrrad: Unfälle mit einem Anprall des Pkw an der Rückseite des Fahrrades wiesen einen höheren Anteil der Schwerverletzten auf.

Die Verletzungsrisikofunktionen für Radfahrer wurde ausgehend von der vektoriellen Geschwindigkeitsänderung des Radfahrers für das Auftreten von Verletzungen der Schweren MAIS 2+ und MAIS 3+ beschrieben. Am Beispiel der Verletzungsrisikofunktion in Abhängigkeit des Alters des verunglückten Radfahrers ließ sich erkennen, dass bei einer vektoriellen Geschwindigkeitsänderung infolge des Zusammenstoßes mit einem Pkw um über $40 \mathrm{~km} / \mathrm{h}$ eine gegenüber niedrigeren Geschwindigkeitsänderungen steigende Wahrscheinlichkeit von MAIS 3+ - Verletzungen auftrat (Bild 5). Die Abbildung zeigt dabei den Einfluss des Alters auf die Verletzungsschwere in Abhängigkeit der vektoriellen Geschwindigkeitsänderung. ${ }^{10}$ Alle weiteren Einflussgrößen, wie die Anprallrichtung oder die Ortslage des Unfalls blieben konstant. Zu vermuten ist allerdings, dass die Geschwindigkeiten im Radverkehr nur einen kleinen Teil der vektoriellen Geschwindigkeitsänderung bei einer Kollision mit einem Kfz ausmachen und die vektorielle Geschwindigkeitsänderung wesentlich von der Geschwindigkeit des $\mathrm{Kfz}$ bei der Kollision abhängt.

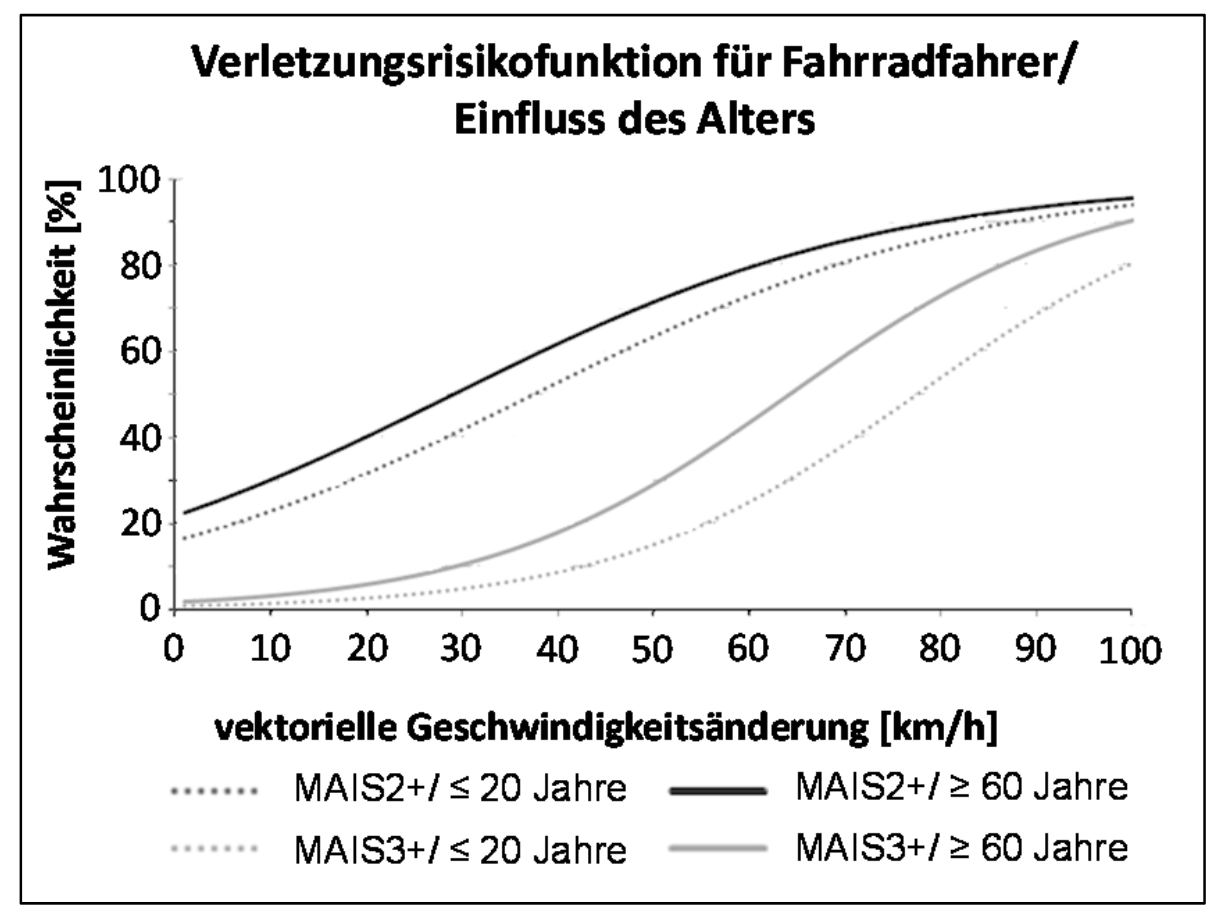

Bild 5: Verletzungsrisikofunktionen für Fahrradfahrer unter Einfluss des Alters (Richter, 2011) schwindigkeit. Er kennzeichnet die Änderung des Bewegungsverhaltens des Fahrzeuges. Die vektorielle Geschwindigkeitsänderung hängt von der Relativgeschwindigkeit, vom Masseverhältnis der Beteiligten und vom Energieabsorptionsvermögen ab. Die Angabe erfolgte in km/h. (Richter, 2011) 
Neben dem Alter und Außerortsunfällen wiesen vor allem Unfälle durch Pkw, welche auf Radfahrer auffuhren, ein besonders hohes Risiko schwerer Verletzungen auf. Unfälle dieses Typs umfassten mit etwa $5 \%$ aber nur einen geringen Teil der in der GIDAS-Datenbank erfassten Radverkehrsunfälle mit Personenschaden (Richter, 2011).

\subsection{Fazit}

Der Radverkehr hat an dem allgemeinen Rückgang der Unfälle mit schwerem Personenschaden seit den 1990er Jahren nicht teilgehabt. Bezogen auf den Bevölkerungsanteil verunglückten Über-65jährige Radfahrer bei Innerortsunfällen überproportional häufig tödlich. Bezogen auf die Verkehrsleistung im Radverkehr, verunglückten insbesondere männliche Kinder und Jugendliche sowie Über-65Jährige im Alter von über 74 häufiger als Radfahrer anderer Altersgruppen.

Radhelmbenutzer erfuhren nach vorliegenden Untersuchungen im Vergleich zu Nichthelmträgern signifikant seltener Kopfverletzungen und insbesondere weniger schwere Kopfverletzungen.

Für Pedelec 25-Fahrer konnten, abgesehen von um 1-2 km/h höheren Durchschnittsgeschwindigkeiten im Vergleich zu Radfahrern, keine wesentlichen Unterschiede im Verkehrs- und Konfliktgeschehen festgestellt werden. Pedelec 45 Fahrer wurden aufgrund der deutlich höheren Fahrgeschwindigkeit bei einem Unfall möglicherweise schwerer verletzt als Fahrrad- oder Pedelec 25-Fahrer. Erste belastbare statistische Unfallzahlen für Pedelec-Fahrer sind frühestens im Jahr 2017 zu erwarten.

Bisherige Untersuchungen zeigten für Straßen mit Radwegen einen Anstieg der Unfalldichte mit den Radverkehrsstärken auf. Bei Radfahr- und Schutzstreifen sowie Fahrbahnführungen wurden keine belastbaren Wirkungen der Radverkehrsstärke auf die Unfalldichten ermittelt. Besondere Gefährdungen bestanden bei Radwegen an Einmündungen und Grundstückszufahrten sowie aus dem regelwidrigen links Fahren von Radfahrern.

Nach früheren Untersuchungen wiesen Fahrbahnführungen des Radverkehrs vergleichbar hohe Unfallraten wie bei Radwegführungen auf. Etwa zwei Drittel der Radfahrerunfälle im Mischverkehr ereigneten sich auf Streckenabschnitten (ohne Einmündungen), etwa ein Drittel an Einmündungen.

Radfahrer-Unfälle an lichtsignalgeregelten Knotenpunkten hatten bei Führung des Radverkehrs auf der Fahrbahn einen hohen Anteil an allen Radfahrer-Unfällen. Hier ereigneten sich v. a. Abbiegenund Einbiegen-/ Kreuzen-Unfälle. An lichtsignalgeregelten Knotenpunkten bewirkte insbesondere eine vollständige signaltechnische Sicherung des Radverkehrs eine hohe Sicherheit gegenüber Abbiegeunfällen. Kann keine vollständige signaltechnische Sicherung eingerichtet werden, bieten an lichtsignalgeregelten Knotenpunkten Führung auf der Fahrbahn oder auf Radfahrstreifen Sicherheitsvorteile gegenüber Führungen auf Radwegen. Bei Radwegen waren insbesondere Sichthindernisse in den Zufahrten ein wesentlicher Einflussfaktor der Unfallzahlen.

Die Durchschnittsgeschwindigkeit von Radfahrern wurde in einer Untersuchung aus dem Jahr 1992

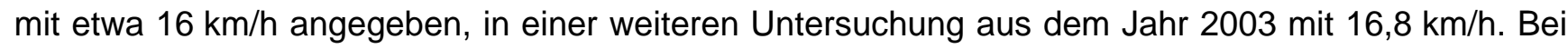
einer vektoriellen Geschwindigkeitsänderung infolge eines Zusammenstoßes mit einem Pkw um über $40 \mathrm{~km} / \mathrm{h}$ stieg die Wahrscheinlichkeit schwerer Verletzungen (MAIS 3+ - Verletzungen) von Radfahrern im Vergleich zu niedrigeren Geschwindigkeitsänderungen. Zukünftig höhere Geschwindigkeiten im Radverkehr könnten hierbei einen negativen Effekt haben. 
Im Hinblick auf die zu erwartenden Änderungen des Radverkehrs bestehen erhebliche Kenntnisdefizite:

- Der demografische Wandel mit einem höheren Anteil von Über-65-Jährigen am Radverkehr könnte zu einer Zunahme von Radfahrer-Unfällen und insbesondere zu mehr schweren Unfällen führen. Für den heutigen Radverkehr ist der Kenntnisstand über das Unfallrisiko verschiedener Altersgruppen, über die Unfallfolgen und mögliche typische Unfallverläufe gerade Über-65-Jähriger jedoch noch gering.

- Auch über Einflüsse wachsender Radverkehrsstärken auf die Anzahl von Radfahrer-Unfällen war der Kenntnisstand insgesamt noch gering. Offen ist u. a.,

- ob der für heutige Radwege ermittelte Anstieg der Unfalldichten mit der Radverkehrsstärke einen besonderen Handlungs- und Sicherungsbedarf nur für Radwege begründet und

- $\quad$ ob die in früheren Untersuchungen mit vergleichsweise kleinen Kollektiven gewonnene Erkenntnis, dass bei Radfahrstreifen-, Schutzstreifen oder Mischverkehrsführungen keine derartigen Einflüsse bestehen, verallgemeinerbar ist.

- Insbesondere über mögliche Einflüsse der Radverkehrsstärken auf die Schwere und die Typen von Radfahrerunfällen lagen bislang keine Erkenntnisse vor.

- Offen war, ob sich ein Anstieg der Radfahrergeschwindigkeiten, wie er aus den Untersuchungen aus den Jahren 1992 und 2003 hervorgeht, fortgesetzt hat. Über mögliche Einflüsse höherer Geschwindigkeiten auf die Anzahl und die Schwere von Radfahrer-Unfällen lagen bislang ebenso keine Erkenntnisse vor.

- Auch über die Regelbefolgung durch Radfahrer unterschiedlicher Altersgruppen und mögliche Einflüsse der Radverkehrsstärken auf die Regelbefolgung bestanden Kenntnislücken. 


\section{Methodik der Untersuchung}

\subsection{Kurzbeschreibung der Arbeitsschritte}

Auf 192 Streckenabschnitten mit unterschiedlichen Radverkehrsführungen und -knoten wurden Verkehrszählungen und Verhaltensbeobachtungen für 108.677 Radfahrer sowie Geschwindigkeitsmessungen für 19.000 Radfahrer durchgeführt. In einer makroskopischen Analyse (Makroanalyse) wurden 900 Radfahrerunfälle mit Personenschaden der Jahre 2009-2011 (U(P)/3a) ausgewertet. Diese Unfälle wurden auch für die Erstellung eines multikriteriellen Unfallmodells herangezogen. Eine mikroskopische Unfallanalyse umfasste 98 Streckenabschnitte mit unterschiedlichen Radverkehrsführungen und 407 Radfahrerunfälle mit Personenschaden der Jahre 2009-2011.

Weiterhin wurden 606 Radfahrerunfälle mit Personenschaden (Jahre 2009-2011) an 218 lichtsignalgeregelten Knotenpunkten mit unterschiedlichen Radverkehrsführungen in einer makroskopischen Analyse (Makroanalyse) ausgewertet. Eine mikroskopische Unfallanalyse umfasste 448 Knotenarme. Bei 164 Knotenarmen konnten die Stärken und demografische Zusammensetzungen des Radverkehrs auf den angrenzenden zuführenden Streckenabschnitten zugeordnet werden, hier wurden 179 Unfälle mit Personenschaden untersucht. An 77 Zufahrten zu lichtsignalgeregelten Knotenpunkten wurden außerdem die Fahrlinien und Rotlichtverstöße von 5.074 Radfahrern mit Videotechnik für jeweils eine Stunde ausgewertet.

In sechs Szenarien wurden anschließend mögliche Entwicklungstendenzen des Radverkehrs entwickelt und deren Auswirkungen auf die Verkehrssicherheit abgeschätzt. Auf Basis einer zusammenfassenden Bewertung der Untersuchungsergebnisse wurden Folgerungen gezogen für erforderliche Anpassungen an der Infrastruktur (Strecke und Knotenpunkten), um unter den veränderten Rahmenbedingungen des künftigen Radverkehrs eine hohe Verkehrssicherheit gewährleisten zu können. 
Das Bild 6 gibt einen Überblick über die Arbeitsschritte der Untersuchung.

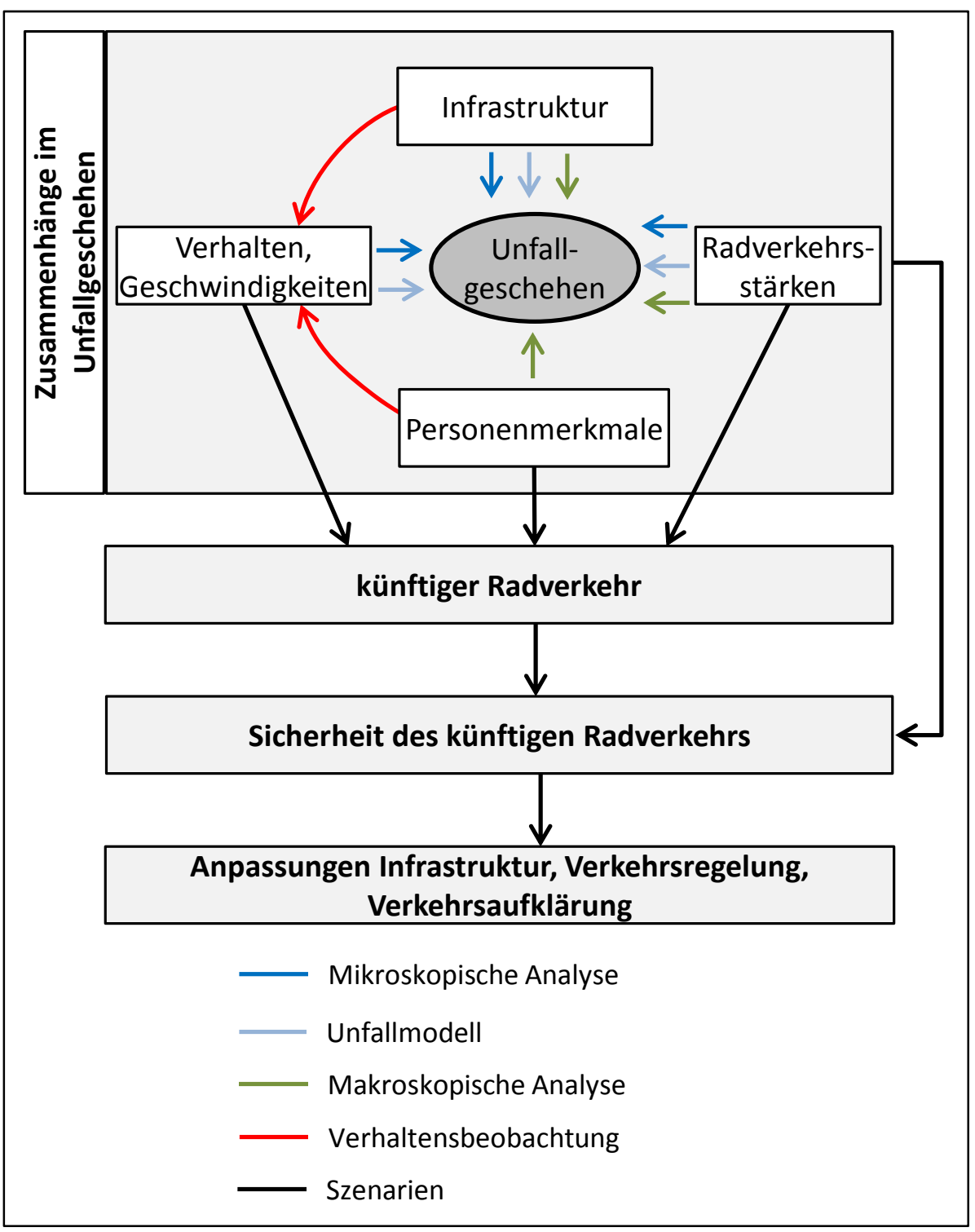

Bild 6: Struktogramm der Arbeitsschritte 


\subsection{Fragestellungen}

Aus den offenen Fragen der Literaturanalyse ließen sich unter Berücksichtigung der Ziele des Projektes die folgenden Untersuchungsfragen ableiten:

1. Welchen Einfluss hat das (zukünftige) Radverkehrsaufkommen auf die Verkehrssicherheit im Radverkehr?

1.1. Welchen Einfluss haben Veränderungen in folgenden Bereichen auf die Verkehrssicherheit?

1.1.1. Höheres Radverkehrsaufkommen

1.1.2. Zusammensetzung der Altersgruppen

1.1.3. Geschwindigkeiten

1.1.4. Verbreitung von Pedelecs (bzw. verschiedener Fahrradtypen im Allgemeinen)

1.1.5. Veränderungen im Verkehrsverhalten (Regelakzeptanz)?

2. Wie kann die Radverkehrsinfrastruktur bei verändertem Radverkehrsaufkommen verkehrssicher gestaltet werden?

2.1. Welchen Einfluss haben die o. g. Veränderungen auf die unterschiedliche Infrastruktur?

2.1.1. Auf der Strecke (inklusive nicht lichtsignalgeregelter Anschlussknoten)

2.1.2. An lichtlichtsignalgeregelten Knotenpunkten

2.2. Welche Anpassungen an der Infrastruktur (Strecke und Knotenpunkte) sind notwendig, um unter den veränderten Rahmenbedingungen eine hohe Verkehrssicherheit gewährleisten zu können?

2.2.1. Welche Radverkehrsanlagen sind grundsätzlich geeignet und welche nicht?

2.2.2. Welche Anpassungen sind nötig?

\subsection{Untersuchungsbeispiele}

Das Untersuchungskollektiv umfasste 165 Streckenabschnitte mit Mischverkehr, Radwegen, Radfahroder Schutzstreifen sowie mit Radverkehrsführungen auf Bussonderfahrstreifen in angebauten Straßen mit einer zulässigen Höchstgeschwindigkeit von $50 \mathrm{~km} / \mathrm{h}$. Neben diesen Anlagentypen an Verkehrsstraßen wurden Fahrradstraßen und selbständige Wege einbezogen. Fast alle Streckenabschnitte wiesen beidseitig gleiche Anlagentypen bzw. in beiden Fahrtrichtungen gleiche Führungsformen auf. Die Abschnitte waren zwischen 400 und 700 m lang und bezogen auch Einmündungen (Anschlussknoten) und Grundstückszufahrten ein. Die Radwege und einzelne gemeinsame Führungen des Rad- und Fußverkehrs wurden in Einrichtungsverkehr betrieben. Die Tabelle 4 zeigt die Anzahl der Untersuchungsstrecken nach den einbezogenen Städten.

In der Untersuchung wurden über die deutschen Städte hinaus Verhaltensbeobachtungen an 14 Streckenabschnitten mit Radwegen, 4 mit Radfahrstreifen und 10 mit Schutzstreifen (Straßenseiten) in Groningen (Niederlande) durchgeführt. 
Tabelle 4: Anzahl der Untersuchungsstrecken in den deutschen Städten (Straßenseiten)

\begin{tabular}{|c|c|c|c|c|c|c|c|c|c|c|c|}
\hline & \multicolumn{10}{|c|}{ Führung des Radverkehrs } & \multirow[b]{2}{*}{ 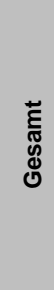 } \\
\hline & 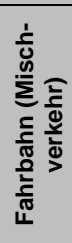 & 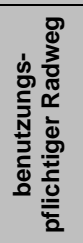 & 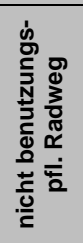 & 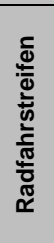 & 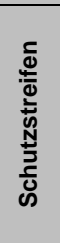 & 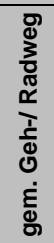 & 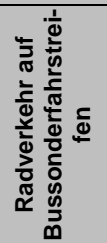 & 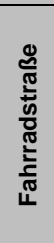 & 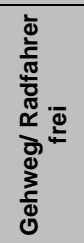 & 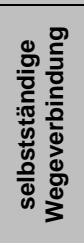 & \\
\hline Berlin & 2 & 6 & 2 & 13 & 11 & & 12 & 6 & & & 52 \\
\hline Bonn & & & & 6 & 14 & & & & & & 20 \\
\hline Dresden & 16 & 17 & & 10 & 7 & 2 & & & 2 & & 54 \\
\hline Fürth & & 2 & 2 & 4 & & & & & & & 8 \\
\hline Halle (Saale) & & 16 & 2 & 6 & & & & & & 6 & 30 \\
\hline Hannover & 14 & 18 & 14 & & 2 & & & 6 & & & 54 \\
\hline Kiel & & & & 2 & 4 & & & 8 & & & 14 \\
\hline Köln & & 10 & 4 & 4 & 6 & & & & & & 24 \\
\hline Leipzig & 15 & 19 & & 11 & 10 & & & & 1 & 4 & 60 \\
\hline Münster & 2 & 4 & & 2 & 2 & & & 24 & & & 34 \\
\hline Nürnberg & & 8 & 8 & 14 & & & & & & & 30 \\
\hline Troisdorf & & & & & 4 & & & & & & 4 \\
\hline Gesamt & 49 & 100 & 32 & 72 & 60 & 2 & 12 & 44 & 3 & 10 & 384 \\
\hline
\end{tabular}

Neben den Streckenabschnitten wurden 218 lichtsignalgeregelte Knotenpunkte mit 747 Knotenzufahrten einbezogen. Das Kollektiv umfasste überwiegend Fahrbahn- oder Radwegführungen in den Zufahrten. Mit etwas kleineren Kollektiven waren Radfahrstreifen oder Schutzstreifen in den einzelnen Zufahrten repräsentiert (Tabelle 5).

Tabelle 5: Führung des Radverkehrs in den Knotenzufahrten (Anzahl der Knotenzufahrten)

\begin{tabular}{|l|c|}
\hline Führung des Radverkehrs & Anzahl Zufahrten \\
\hline Fahrbahn & 248 \\
\hline benutzungspflichtiger Radweg & 247 \\
\hline Radfahrstreifen & 98 \\
\hline Schutzstreifen & 70 \\
\hline Gem. Führung Radfahrer und Fußgänger & 22 \\
\hline Schutzstreifen und nicht benutzungspflichtiger Radweg & 8 \\
\hline Geradeaus-Radfahrstreifen neben Kfz-Rechtsabbiegestreifen & 7 \\
\hline Vorbeifahrstreifen & 6 \\
\hline nicht benutzungspflichtiger Radweg & 41 \\
\hline Gesamtsumme & 747 \\
\hline
\end{tabular}

Die Tabelle 6 auf Seite 35 gibt einen Überblick über die baulichen und betrieblichen Merkmale der Untersuchungsbeispiele, die bei Ortsbesichtigungen erhoben wurden. 


\subsection{Ermittlung von Stärken, Zusammensetzung und Verhaltensmerk- malen des Radverkehrs}

\subsubsection{Zählungen und Verhaltensmerkmale}

Auf den Untersuchungsstrecken wurden bei vierstündigen Zählungen (7-11 Uhr bzw. 14-18 Uhr)

- die Verkehrsstärken des Radverkehrs (108.677 Radfahrer),

- die Altersgruppe nach Augenschein und das Geschlecht der Radfahrer,

- die Fahrradtypen ${ }^{11}$ sowie

- ausgewählte Verhaltensmerkmale

erfasst (vgl. Tabelle 6).

Aus den Zählungen wurden die täglichen Radverkehrsstärken mit einem Verfahren zur Hochrechnung von Kurzzeitzählungen des Radverkehrs (Schiller et al. 2011) abgeleitet. Die Radverkehrsstärken dienten damit der Bestimmung verkehrsleistungsbezogener Kenngrößen wie Unfallraten und Unfallkostenraten (Frage 1.1.1).

Die Flächennutzung und die Fahrtrichtungen der Radfahrer wurden

- auf die Regelverstöße bei den verschiedenen Radverkehrsanlagen hin untersucht (Frage 1.1.5),

- nach Altersgruppen differenziert (Fragen 1.1.2 und 1.1.5) und

- als Eingangsvariable eines multikriteriellen Unfallmodells und der mikroskopischen Unfallanalysen herangezogen (Fragen 1.1.1 bis 1.1.5).

\subsubsection{Verhaltensbeobachtungen an lichtsignalgeregelten Knotenpunkten}

An 77 Zufahrten zu lichtsignalgeregelten Knotenpunkten wurden die Fahrlinien und Rotlichtverstöße von 5.074 Radfahrern mit Videotechnik für jeweils eine Stunde ausgewertet. Dazu wurden die Radfahrer in der Knotenzufahrt gefilmt, so dass die Signalisierung, der weitere Fahrverlauf sowie weitere Merkmale erfasst wurden. Die Ergebnisse der Verhaltensbeobachtungen wurden in dem vorliegenden Bericht mit Schwerpunkt auf Fehlverhaltensweisen von Radfahrern und möglichen Einflüssen auf die Radfahrerunfälle betrachtet (Fragen 1.1.2 und 1.1.5). 


\subsection{Ermittlung von Geschwindigkeiten im Radverkehr}

An den Streckenabschnitten wurden die Geschwindigkeiten von etwa 19.000 Radfahrern mit Videoaufnahmen bzw. mit Stoppuhren gemessen. Die Geschwindigkeiten wurden in Differenzierung nach der

- Altersgruppe und dem Geschlecht der Radfahrer,

- den Fahrradtypen sowie

- der genutzten Verkehrsfläche und der Fahrtrichtung

ausgewertet und mit früheren Untersuchungen abgeglichen. Mit uni- und multivariaten Analysen wurden wesentliche Einflüsse der Anlagentypen, der Altersgruppen und der Fahrradtypen auf die Geschwindigkeiten herausgearbeitet. Mögliche Einflüsse der Geschwindigkeiten auf die Verkehrssicherheit wurden im Rahmen der makroskopischen Unfallanalyse, des Unfallmodells und der mikroskopischen Unfallanalyse geprüft (Frage 1.1.3 und Frage 2.2.1). 


\section{6 Übersicht der einbezogenen Variablen}

Die in die Untersuchung einbezogenen Variablen sind in Tabelle 6 und Tabelle 7 zusammengestellt.

Tabelle 6: Übersicht der aufgenommenen Variablen (baulich-betriebliche Merkmale und Verhaltensmerkmale)

\begin{tabular}{|c|c|}
\hline \multicolumn{2}{|l|}{ Baulich-betriebliche Merkmale } \\
\hline \multirow{15}{*}{$\begin{array}{l}\text { Baulich-betriebliche Merkmale Streckenab- } \\
\text { schnitte }\end{array}$} & Radverkehrsanlage \\
\hline & Breite Radverkehrsanlage \\
\hline & Städtebauliche Nutzung \\
\hline & Kfz-Parken \\
\hline & Breite Sicherheitstrennstreifen zu Parkstreifen \\
\hline & Art und Führung ÖPNV \\
\hline & Breite Fahrgasse \\
\hline & Breite Sicherheitstrennstreifen zu Fahrbahn \\
\hline & Befahrbarkeit aufgrund des Belags \\
\hline & Mängel Linienführung \\
\hline & Breite Gehweg \\
\hline & Trennung Rad-/Gehweg \\
\hline & $\begin{array}{l}\text { Mängel an Anschlussknoten und stark befahrenen Grundstückszufahrten (z. B. Sichtfeld einbiegende } \\
\text { Kfz oder Sicht RW - FB eingeschränkt) }\end{array}$ \\
\hline & Streckenlänge \\
\hline & Kfz-Verkehrsstärken \\
\hline \multirow[t]{17}{*}{ Baulich-betriebliche Merkmale LSA-Knoten } & Führung Zufahrt \\
\hline & Wechsel Radverkehrsführung in Zufahrt \\
\hline & Führung Ausfahrt \\
\hline & Furt markiert \\
\hline & Furt flächig eingefärbt \\
\hline & Furtabsetzung von den parallelen Kfz-Fahrstreifen \\
\hline & Führung Linksabbieger \\
\hline & Besondere Vorkehrungen Rechtsabbieger \\
\hline & Sichtfeld in Zufahrt \\
\hline & Signalisierung Zufahrt \\
\hline & Raumvorlauf Zufahrt \\
\hline & Zeitvorlauf vor Grünphase rechts abbiegender Kfz \\
\hline & Rotzeiten Geradeausfahrt \\
\hline & Wartezeit auf Insel bei Geradeausfahrt \\
\hline & gleichzeitige Freigabe für $\mathrm{R}$ und aus gleicher Zufahrt rechts abbiegende Kfz \\
\hline & gleichzeitige Freigabe für R und aus gegenüberliegender Zufahrt links abbiegende Kfz \\
\hline & Kfz-Verkehrsstärken \\
\hline \multicolumn{2}{|l|}{ Verhaltensmerkmale } \\
\hline \multirow{10}{*}{$\begin{array}{l}\text { Verhaltensbeobachtungen und Messungen } \\
\text { an Strecken }\end{array}$} & Altersgruppe (augenscheinlich: < 10 Jahre (J.), 10-14 J., 15-17 J.,18-24 J., 25-65 J. sowie > 65 J.) \\
\hline & Geschlecht (männlich, weiblich) \\
\hline & $\begin{array}{l}\text { Fahrradtyp (City/ Stadt/ Standardrad, Mountainbike, Rennrad, Lastenrad, Pedelec 25, Hollandrad } \\
\text { u. a. m.) }\end{array}$ \\
\hline & $\begin{array}{l}\text { Führungsform des Radverkehrs (Fahrbahn, benutzungspflichtiger Radweg, nicht benutzungspfl. Rad- } \\
\text { weg, Radfahrstreifen, Schutzstreifen, gem. Geh-/ Radweg, Radverkehr auf Bussonderfahrstreifen, } \\
\text { Fahrradstraße, Gehweg/ Radfahrer frei, selbstständige Wegeverbindung) }\end{array}$ \\
\hline & Kfz-Verkehrsstärken \\
\hline & Geschwindigkeiten \\
\hline & Radverkehrsstärken \\
\hline & Flächennutzung \\
\hline & Linksfahrten \\
\hline & Helmnutzung \\
\hline \multirow{4}{*}{$\begin{array}{l}\text { Verhaltensbeobachtung an lichtsignalgere- } \\
\text { gelten Knotenpunkten }\end{array}$} & Radverkehrsführung \\
\hline & Fahrlinien \\
\hline & Rotlichtverstöße \\
\hline & Altersgruppe \\
\hline
\end{tabular}


Tabelle 7: Übersicht der aufgenommenen Variablen (Unfallanalysen)

\begin{tabular}{|l|l|}
\hline Unfallanalysen & \\
\hline \multirow{4}{*}{$\begin{array}{l}\text { Variablen der makroskopischen Unfallunter- } \\
\text { suchung }\end{array}$} & Anzahl der Unfälle \\
\cline { 2 - 2 } & Kategorien/ Folgen \\
\cline { 2 - 2 } & Typen/ Arten \\
\cline { 2 - 2 } & Ursachen (von und gegenüber Radfahrern) und Schuldfrage \\
\cline { 2 - 2 } & Unfallgegner \\
\cline { 2 - 2 } & Alter und Geschlecht \\
\cline { 2 - 2 } & Charakteristik und Besonderheiten der Unfallstelle \\
\hline \multirow{5}{*}{$\begin{array}{l}\text { Variablen der mikroskopischen Unfallunter- } \\
\text { suchung }\end{array}$} & Unfallhergangsbeschreibung und/oder dreistellige Unfalltypen \\
\hline
\end{tabular}

\subsection{Unfallanalysen}

\subsubsection{Makroskopische Unfallanalysen - Kenngrößen der Radverkehrsunfälle für Streckenabschnitte und Knotenpunkte}

Im Vordergrund der Untersuchung des zukünftigen Radverkehrs stand in der Unfallanalyse

- die Ermittlung von Einflüssen der Radverkehrsstärken auf die Unfallanzahl (Untersuchungsfrage 1.1.1),

- die Beschreibung von Auffälligkeiten im Unfallgeschehen älterer Verkehrsteilnehmer (Frage 1.1.2) und

- die Überprüfung von Einflüssen der Radfahrergeschwindigkeiten (Frage 1.1.3).

Auf Grundlage der in digitalen Verzeichnissen verfügbaren Radverkehrsunfälle der Kategorien 1-3 wurden

- die Unfalldichte und Unfallrate sowie die Unfallkostendichten und -raten der Streckenabschnitte und

- die Unfalldichten und Unfallkostendichten der Knotenpunkte

errechnet. Die Unfallkostendichten und -raten berücksichtigen sowohl die Anzahl als auch die Schwere der Unfälle. Hierfür wurden die Unfallkostensätze der BASt (Preisstand 2010) herangezogen und angepasst (Kap. 7.2 im Anhang).

Das Kollektiv umfasste 956 Radfahrerunfälle mit Personenschaden der Jahre 2009-2011 an Streckenabschnitten ${ }^{12}$ und 624 Radfahrerunfälle mit Personenschaden (Jahre 2009-2011) an den lichtsignalgeregelten Knotenpunkten. Die im Kollektiv vorgefundenen Unfälle älterer Verkehrsteilnehmer wurden auf Auffälligkeiten bezüglich:

- Unfalltyp,

- Unfallursache,

- Radverkehrsanlagentyp sowie

- Hauptverursacher (Fragen 1.1.2, 2.1.1., 2.1.2 und 2.2.1)

12 Als Strecke wurde der Bereich zwischen Verkehrsknotenpunkten definiert. Das Streckenkollektiv enthält demnach auch Anschlussknotenpunkte und Zufahrten, sodass die Kollektivgröße keinen Rückschluss auf die Verteilung der Radfahrerunfälle im Straßenraum zulässt. Die tatsächliche Verteilung der Radfahrerunfälle im Straßenraum kann dem Abschnitt 4.1.5 entnommen werden. 
untersucht. Über die Unfälle an den untersuchten Streckenabschnitten und lichtsignalgeregelten Knotenpunkten hinaus wurden für einzelne Fragestellungen auch Unfalldaten aller Radverkehrsunfälle mit Personenschaden in den Städten Berlin, Dresden, Chemnitz, Leipzig, Magdeburg, Halle, Münster, Troisdorf, Bonn und Köln herangezogen. Diese umfassten 12.457 Radfahrerunfälle mit Personenschaden an Streckenabschnitten und 15.520 Radfahrerunfälle mit Personenschaden an lichtsignalgeregelten Knotenpunkten der Jahre 2009-2011.

\subsubsection{Multikriterielle Unfallmodelle}

Im Mittelpunkt der Analyse stand der Aufbau von multikriteriellen Unfallmodellen auf Basis von verallgemeinerten linearen Modellen. Diese können spezifische Einflüsse auf das Unfallgeschehen isoliert quantifizieren. Durch die Herausarbeitung dieser signifikant wirkenden Einflüsse auf das Unfallgeschehen, lässt sich verändertes Unfallgeschehen infolge veränderter (zukünftiger) Rahmenbedingungen prognostizieren.

Das Unfallmodell konzentrierte sich auf Streckenabschnitte mit Einmündungen (Anschlussknoten). Das Unfallmodell bestimmte die erwartete Unfallanzahl in Abhängigkeit der signifikant wirkenden Einflüsse. Im Unfallmodell wurden dabei die in Anhang 7.1.4 genannten Streckenmerkmale sowie die Geschwindigkeitskenngrößen, die Flächennutzung und die Fahrtrichtungen überprüft. Zugleich wurde der Einfluss unterschiedlicher Radfahrerkollektive (Altersstruktur) bestimmt (Fragen 1.1.1, 1.1.2, 1.1.3, 1.1.5)

Die Einflussfaktoren wurden mit Exponential- bzw. Potenzfunktionen multiplikativ miteinander verknüpft. Der Aufbau des Modells erfolgte schrittweise. Das Modell ohne Einflussfaktoren (Nullmodell) beschreibt den Mittelwert der Unfälle des Untersuchungskollektivs. Anschließend wurde jede einzelne zur Verfügung stehende Einflussgröße einzeln in das Modell aufgenommen und auf ihren Erklärungsanteil der systematischen Streuung überprüft. Nach der Aufnahme aller signifikanter Einflussgrößen wurde das Modell auf

- die Anpassung an die Stichprobe und

- daraufhin, ob die enthaltenen Aussagen verallgemeinert werden können,

geprüft. Hierbei kamen Residuen- und Wahrscheinlichkeitsplots zur Anwendung. Die Zielverteilung des Modells basierte auf einer Poissonverteilung. Eine detaillierte Beschreibung der Vorgehensweise kann Kapitel 7.1 im Anhang entnommen werden. 


\subsubsection{Vertiefungsanalyse kleinräumiger Einflussgrößen (mikroskopische Unfallanalyse)}

Für 98 Untersuchungsstrecken und 407 Radfahrerunfälle mit Personenschaden (Unfalljahre 2009-2011) wurden die Unfallhergangsbescheibungen der Polizei in die Auswertung einbezogen. Die Unfälle wurden dem dreistelligen Unfalltypenkatalog des M Uko (FGSV, 2012) zugeordnet und auf die Fahrtrichtung sowie die von den Radfahrern genutzte Verkehrsfläche hin ausgewertet.

Die mikroskopische Unfallanalyse umfasste weiterhin 448 Knotenarme. Hiervon konnten bei 164 Knotenarmen die Stärken und Zusammensetzungen des Radverkehrs auf den angrenzenden zuführenden Streckenabschnitten zugeordnet werden, hier wurden 179 Unfälle mit Personenschaden untersucht.

Die Mikroanalyse thematisierte u. a.

- mögliche punktuellen Häufungen bestimmter Unfalltypen und Einflüssen von InfrastrukturmerkmaIen (insbesondere Untersuchungsfragen 2.2.1, 2.2.2),

- die Flächennutzung der verunglückten Radfahrer im Abgleich mit der beobachteten Flächennutzung (insbesondere Frage 1.1.5),

- die Fahrtrichtung verunglückter Radfahrer, insbesondere im Hinblick auf die beobachtete regelwidrige linke Fahrtrichtung (insbesondere zu Frage 1.1.5),

- Regelübertretungen verunglückter Radfahrer an lichtsignalgeregelten Knoten (wie z. B. regelwidrige Nutzung linker Furten) im Abgleich mit bei den Verhaltensbeobachtungen erfassten Fahrlinien und Regelübertretungen (insbesondere Frage 1.1.5) sowie

- Auffälligkeiten der Unfallhergänge für bestimmte Altersgruppen (insbesondere Frage 1.1.5).

\subsection{Künftige Entwicklung des Radverkehrs und der Radverkehrs- sicherheit}

Für den künftigen Radverkehr sind eine Veränderung der Altersstruktur von Radfahrern, höhere Radverkehrsstärken und eine Veränderung der genutzten Fahrradtypen zu erwarten. Nach dem bisherigen Erkenntnisstand könnten

- der demografische Wandel mit einem höheren Anteil von Über-65-Jährigen am Radverkehr zu einer Zunahme von Radfahrer-Unfällen und insbesondere zu mehr schweren Unfällen führen und

- der für einige Anlagentypen ermittelte Anstieg der Unfalldichten mit der Radverkehrsstärke einen besonderen Handlungs- und Sicherungsbedarf begründen.

Offen ist, ob sich ein Anstieg der Radfahrergeschwindigkeiten auf die Anzahl und die Schwere von Radfahrer-Unfällen auswirkt. 
Mögliche Auswirkungen auf die Verkehrssicherheit wurden daher auf Grundlage der Ergebnisse der oben genannten Untersuchungen für den heutigen Radverkehr nach den Hypothesen in Tabelle 8 geprüft.

Tabelle 8: Hypothesen zur Entwicklung der Radverkehrssicherheit

\begin{tabular}{|c|c|c|c|}
\hline \multicolumn{2}{|c|}{$\begin{array}{l}\text { Fragestellung: } \\
\text { Einfluss aus den u. g. Entwicklungen auf die Rad- } \\
\text { verkehrssicherheit }\end{array}$} & \multicolumn{2}{|c|}{ Hypothesen } \\
\hline 1.1 .1 & Höheres Radverkehrsaufkommen & $\mathrm{H} 1$ & Höhere Radverkehrsstärken bedingen höhere Unfallbelastungen \\
\hline \multirow[t]{3}{*}{1.1 .2} & \multirow{3}{*}{$\begin{array}{l}\text { Veränderte Zusammensetzung der } \\
\text { Altersgruppen }\end{array}$} & $\mathrm{H} 2$ & Mehr ältere Radfahrer bedingen eine Zunahme von Unfällen \\
\hline & & $\mathrm{H} 3$ & Mehr ältere Radfahrer bedingen eine Zunahme schwerer Unfälle \\
\hline & & $\mathrm{H} 4$ & Mehr ältere Radfahrer bedingen andere Unfalltypen und Unfallverläufe \\
\hline \multirow[t]{2}{*}{1.1 .3} & \multirow[t]{2}{*}{ Geschwindigkeiten } & $\mathrm{H} 5$ & Höhere Geschwindigkeiten im RV bedingen mehr Unfälle \\
\hline & & $\mathrm{H} 6$ & Höhere Geschwindigkeiten im RV bedingen schwerere Unfälle \\
\hline \multirow[t]{2}{*}{1.1 .4} & \multirow[t]{2}{*}{$\begin{array}{l}\text { Verbreitung von Pedelecs (bzw. ver- } \\
\text { schiedener Fahrradtypen im Allgemei- } \\
\text { nen) }\end{array}$} & $\mathrm{H} 7$ & $\begin{array}{l}\text { In Zusammenhang mit H 3: Höhere Geschwindigkeiten im RV durch zunehmende } \\
\text { Pedelec-Nutzung bedingen mehr/schwerere Unfälle }\end{array}$ \\
\hline & & H 8 & $\begin{array}{l}\text { In Zusammenhang mit H } 2 \text { und H 7: Zunehmende Geschwindigkeiten von Über-65- } \\
\text { Jährige auf Pedelecs bedingen mehr/schwerere Unfälle }\end{array}$ \\
\hline \multirow[t]{3}{*}{1.1 .5} & \multirow[t]{3}{*}{ Veränderungen im Verkehrsverhalten } & $\mathrm{H} 9$ & $\begin{array}{l}\text { Bei zunehmendem Radverkehrsstärken fahren weniger Radfahrer regelwidrig in linker } \\
\text { Richtung }\end{array}$ \\
\hline & & H 10 & $\begin{array}{l}\text { Mehr ältere Radfahrer bedingen bei Fahrbahnführung, Radfahrstreifen oder Schutz- } \\
\text { streifen eine häufigere Nutzung von Gehwegen }\end{array}$ \\
\hline & & H 11 & $\begin{array}{l}\text { Zunehmende Regelverstöße bewirken mehr Unfälle (regelwidriges Linksfahren, } \\
\text { Nutzung linker Furten an lichtsignalgeregelten Knoten, Rotlichtübertretungen an LSA- } \\
\text { Knoten) }\end{array}$ \\
\hline \multirow[t]{4}{*}{2.2 .1} & \multirow[t]{4}{*}{$\begin{array}{l}\text { Welche Radverkehrsanlagen sind } \\
\text { grundsätzlich geeignet und welche } \\
\text { nicht? }\end{array}$} & H 12 & $\begin{array}{l}\text { Die Einsatzgrenzen werden sich mit schnellerem und zunehmenden Radverkehr } \\
\text { zugunsten des Mischverkehrs auf der Fahrbahn verschieben }\end{array}$ \\
\hline & & H 13 & $\begin{array}{l}\text { Die Einsatzgrenzen werden sich mit schnellerem und zunehmenden Radverkehr } \\
\text { zugunsten einer Führung auf Schutzstreifen verschieben }\end{array}$ \\
\hline & & H 14 & $\begin{array}{l}\text { Die Einsatzgrenzen werden sich mit schnellerem und zunehmenden Radverkehr } \\
\text { zugunsten einer Führung auf Radfahrstreifen verschieben }\end{array}$ \\
\hline & & H 15 & $\begin{array}{l}\text { Duale Führungsformen (z. B. Schutzstreifen und „Gehweg/Radfahrer frei“) können } \\
\text { den unterschiedlichen Bedürfnissen gerecht werden. }\end{array}$ \\
\hline \multirow[t]{4}{*}{ 2.2.2. } & \multirow[t]{4}{*}{ Welche Anpassungen sind nötig? } & H 16 & $\begin{array}{l}\text { Die Mindestanforderungen der VwV-StVO für die Anordnung einer Benutzungspflicht } \\
\text { entsprechen in keiner Weise den Anforderungen des modernen Radverkehrs und } \\
\text { sind anzupassen. }\end{array}$ \\
\hline & & H 17 & $\begin{array}{l}\text { Radwege (Einrichtungsverkehr) und Radfahrstreifen müssen mindestens 2,0 m breit } \\
\text { sein. }\end{array}$ \\
\hline & & $\mathrm{H} 18$ & Die Sichtfelder auf bevorrechtigten Radverkehr müssen ausreichend sein. \\
\hline & & H 19 & $\begin{array}{l}\text { Bei Radwegführungen sind an lichtsignalgeregelten Knoten verstärkt konfliktfreie } \\
\text { Schaltungen erforderlich, um Abbiege-Unfälle durch Unterschätzung der Geschwin- } \\
\text { digkeiten von Pedelecs zu vermeiden. }\end{array}$ \\
\hline
\end{tabular}

Auf Grundlage der Hypothesen wurden anschließend die Einflüsse aus der Radverkehrsstärke, dem Alter und den Radfahrergeschwindigkeiten auf die Unfallanzahl, die Unfallschwere, die Unfalltypenverteilung sowie auf die Regelbefolgung ermittelt (Tabelle 9).

Tabelle 9: Untersuchte Einflüsse und Gliederung zur Darstellung der Untersuchungsergebnisse

\begin{tabular}{|c|c|c|c|c|c|c|c|c|}
\hline & \multicolumn{4}{|c|}{ Streckenabschnitte } & \multicolumn{4}{|c|}{ LSA-Knoten } \\
\hline & Unfallanzahl & Schwere & Typen & Regelbefolgung & Unfallanzahl & Schwere & Typen & Regelbefolgung \\
\hline Radverkehrsstärke & $x$ & $x$ & $x$ & $x$ & $x$ & $x$ & $x$ & $x$ \\
\hline Alter & $x$ & $x$ & $x$ & $x$ & $x$ & $x$ & $x$ & $x$ \\
\hline Geschwindigkeit & $x$ & $x$ & $x$ & $x$ & $x$ & $x$ & $x$ & nicht betrachtet \\
\hline
\end{tabular}


Im Anschluss wurden in verschiedenen Szenarien mögliche Entwicklungstendenzen des Radverkehrs entwickelt. In Anlehnung an aktuelle Tendenzen der Bevölkerungsentwicklung, der Fahrradnutzung und der genutzten Fahrradtypen wurden begründete Annahmen für die folgenden Szenarien getroffen (siehe Kapitel 5.1):

S1: $\quad$ Kurzfristig moderate Zunahme des Radverkehrs

S2-A: $\quad$ Mittelfristige Veränderung der Altersstruktur

S2-A-20: Veränderung der Altersstruktur und moderate Zunahme des Radverkehrs

S2-A-20-V: Veränderung der Geschwindigkeiten aufgrund einer veränderten Altersstruktur und veränderter Fahrradtypen bei moderater Zunahme des Radverkehrs

S2-A-40: Veränderung der Altersstruktur und erheblich höhere Stärken des Radverkehrs sowie

S2-A-40-V: Erheblich höhere Stärken und Veränderung der Geschwindigkeiten des Radverkehrs

Unter Zuhilfenahme der Ergebnisse der o. g. Analysen wurden künftige Entwicklungen für die Anzahl und die Schwere von Radverkehrsunfällen abgeschätzt. Ziel war dabei keine exakte Prognose, sondern ein Aufzeigen künftiger Entwicklungen der Radverkehrssicherheit, die sich in der heutigen Anzahl und Charakteristik von Radverkehrsunfällen begründen

\subsection{Schlussfolgerungen}

Auf Basis einer zusammenfassenden Bewertung der Untersuchungsergebnisse wurden Schlussfolgerungen gezogen für ggf. erforderliche Anpassungen an der Infrastruktur (Strecke und Knotenpunkte), um unter den veränderten Rahmenbedingungen eine hohe Verkehrssicherheit gewährleisten zu können. Bewertet wurden:

- die grundsätzliche Eignung verschiedener Radverkehrsanlagen,

- die ggf. erforderliche Anpassungen der Planung, des Entwurfs und des Betriebs der Radverkehrsanlagen,

- der Anpassungsbedarf bei technischen Regelwerken,

- Anpassungen von Regelungen des Straßenverkehrsrechts sowie

- Anpassungen der strategischen Ausrichtung der Verkehrsaufklärung. 


\section{Einfluss von Radverkehrsstärken, Alter und Geschwindigkeiten von Radfahrern auf die Verkehrssicherheit}

\subsection{Kenngrößen des heutigen Radverkehrs:}

\subsubsection{Altersgruppen im Radverkehr und genutzte Fahrradtypen}

\section{Kollektiv der Zählungen und Verhaltensbeobachtungen}

Im bundesweiten Durchschnitt hat der Radverkehr nach der Untersuchung MiD 2008 (BMVBS, 2010) einen Anteil von $11 \%$ an der Zahl aller Wege. In den Städten, die an dem System repräsentativer Verkehrserhebungen (SrV, 2008) teilgenommen haben, hat der Radverkehr im Mittel einen Anteil von $13 \%$ am Gesamt- und von $15 \%$ am Binnenverkehr. Die Modal-Split-Anteile nach dem SrV sind für den Stadtverkehr deutscher Städte verallgemeinerbar.

Etwa $68 \%$ der bei den Verhaltensbeobachtungen dieser Untersuchung erfassten 109.000 Radfahrer waren 25-65 Jahre alt, etwa 22 \% 18-24 Jahre. Über-65-Jährige stellten etwa $4 \%$ der beobachteten Radfahrer (Tabelle 10). Die Anteile der augenscheinlich 25- bis 65-Jährigen waren mit den Anteilen dieser Altersgruppe an den Radverkehrs-Fahrleistungen in Großstädten nach der MiD 2008 vergleichbar. Über-65-Jährige waren im Zählkollektiv gegenüber den Anteilen dieser Altersgruppe an den Radverkehrs-Fahrleistungen deutlich schwächer, 18- bis 24-Jährige anteilig deutlich häufiger repräsentiert. Dies kann dadurch begründet sein, dass einige Untersuchungsabschnitte auf Radverkehrsverbindungen zu Universitäten lagen (z. B. Hannover, Dresden) und mehrere Untersuchungsabschnitte in Münster hohe Radverkehrsstärken aufwiesen und Studenten in Münster einen überdurchschnittlich hohen Bevölkerungsanteil von etwa $15 \%$ einnehmen.

Tabelle 10: Altersgruppen der gezählten Radfahrer und Anteile der Altersgruppen an der Verkehrsleistung des Radverkehrs (eigene Berechnung auf Grundlage der Stichprobe der MiD 2008)

\begin{tabular}{|c|c|c|c|c|c|c|}
\hline \multirow{3}{*}{ Altersgruppe } & \multicolumn{3}{|c|}{ Anteil an den gezählten Radfahrern [\%] $(n=108.677)$} & \multirow{2}{*}{\multicolumn{3}{|c|}{$\begin{array}{c}\text { Anteile an der Verkehrsleistung nach MID } 2008 \text { [\%] } \\
\text { Gemeindegrößenklasse }\end{array}$}} \\
\hline & & \multicolumn{2}{|c|}{ davon } & & & \\
\hline & & männlich & weiblich & $\begin{array}{c}50 \text { bis unter 100Tsd. } \\
\text { EW }\end{array}$ & $\begin{array}{c}100 \text { bis unter 500Tsd. } \\
\text { EW }\end{array}$ & Ab 500Tsd. EW \\
\hline < $10 \mathrm{~J}$. & 1,1 & 1 & 1 & 4,6 & 3,5 & 3,3 \\
\hline 10-14 J. & 2,4 & 1 & 1 & 5,1 & 3,4 & 3,8 \\
\hline 15-17 J. & 2,8 & 1 & 1 & 8,0 & 5,2 & 2,7 \\
\hline 18-24. J. & 22,3 & 10 & 12 & 14,9 & 9,9 & 7,3 \\
\hline $25-65 \mathrm{J.}^{13}$ & 67,4 & 38 & 30 & 54,5 & 60,6 & 73,4 \\
\hline$>65 \mathrm{J.}^{13}$ & 4,0 & 3 & 1 & 13,1 & 17,3 & 9,6 \\
\hline k.A. & 0,0 & 0 & 0 & & & \\
\hline Gesamt & 100,0 & 54 & 46 & 100,0 & 100,0 & 100,0 \\
\hline
\end{tabular}


Eine Überprüfung von Startzeitpunkten von Wegen mit dem Fahrrad in Kern- und Großstädten mit mindestens 100.000 Einwohnern nach Altersgruppen auf Basis der MiD 2008 ergab, dass Ältere zu anderen Zeiten mit dem Rad unterwegs sind als jüngere Erwachsene. Das Radverkehrsaufkommen der älteren Radfahrer verzeichnet eine deutliche Aufkommensspitze zwischen 9-13 Uhr. Dieser Umstand wurde bei der Unfallrisikobewertung der einzelnen Altersgruppen (Kapitel 4.3) überprüft. Es zeigte sich, dass sich das Verhältnis der Unfallraten in den einzelnen Altersgruppen zueinander auch bei Ansatz gesonderter Hochrechnungsfaktoren für Über-65-Jährige nicht wesentlich ändert.

$87 \%$ der in dieser Untersuchung beobachteten Radfahrer nutzten ein City- oder Standardrad, etwa $9 \%$ ein Mountainbike und $4 \%$ ein Rennrad. Mit einem Pedelec 25 fuhren 0,3\% der Radfahrer (Tabelle 11). Bis auf die Pedelecs 25 sind die Anteile der gezählten Fahrradtypen mit den Anteilen der Fahrradtypen an den verkauften Fahrrädern vergleichbar.

Tabelle 11: Gezählte und in Betrieb befindliche Fahrradtypen (ZIV, 2013)

\begin{tabular}{|l|c|c|}
\hline & $\begin{array}{c}\text { Anteil an den gezählten } \\
\text { Fahrrädern [\%] }\end{array}$ & $\begin{array}{c}\text { Anteil an den verkauften } \\
\text { Fahrrädern [\%] }\end{array}$ \\
\hline City/Stadt/Standardrad & 87,2 & 75,5 \\
\hline Mountainbike & 8,6 & 10,0 \\
\hline Rennrad & 3,5 & 4,0 \\
\hline Lastenrad & 0,4 & \\
\hline Tandem & 0,0 & 10,0 \\
\hline Pedelec 25 & 0,3 & \\
\hline Liegerad & 0,0 & 0,5 \\
\hline E-Bike (Pedelec 45) & 0,0 & 100,0 \\
\hline Sonstige / . A. & 0,0 & \\
\hline Gesamt & 100,0 & \\
\hline
\end{tabular}

\section{Kollektiv der Geschwindigkeitsmessungen}

Unter den Radfahrern, deren Geschwindigkeiten gemessen wurden, waren 25- bis 65-Jährige als größte Altersgruppe mit einem Anteil repräsentiert, der auch dem Anteil der durch diese Altersgruppe erbrachten Verkehrsleistung in Gemeinden mit mindestens 50.000 Einwohnern entsprach. Kinder und Über-65-Jährige waren um etwa vier Prozentpunkte seltener, junge Erwachsene um fast zehn Prozentpunkte überrepräsentiert (Tabelle 12). ${ }^{14}$

Tabelle 12: Altersgruppen der Radfahrer bei den Geschwindigkeitsmessungen und Anteile der Altersgruppen an der Verkehrsleistung des Radverkehrs (Eigene Berechnung auf Grundlage der korrigierten Stichprobe der MiD 2008)

\begin{tabular}{|c|c|c|}
\hline Altersgruppe & $\begin{array}{l}\text { Anteil an den Radfahrern mit gemesse- } \\
\text { nen Geschwindigkeiten [\%], frei und } \\
\text { nach StVO fahrend (eigene Erhebung) }\end{array}$ & $\begin{array}{c}\text { Anteile an der Verkehrsleistung } \\
\text { (Gemeinden } \geq 50 T s d \text {. EW, MiD, } \\
\text { korrigiert) }\end{array}$ \\
\hline$<10 \mathrm{~J}$. & 0,4 & 4,0 \\
\hline $10-14 \mathrm{~J}$. & 1,4 & 4,0 \\
\hline $15-17 \mathrm{~J}$. & 4,8 & 5,0 \\
\hline $18-24 \mathrm{~J}$. & 19,6 & 10,0 \\
\hline $25-65 \mathrm{~J}$. & 64,2 & 65,0 \\
\hline$>65 \mathrm{~J}$. & 9,6 & 13,0 \\
\hline Gesamt & 100,0 & 100,0 \\
\hline $\mathbf{N}$ & 14.243 Rad & 25.615 Pkm \\
\hline
\end{tabular}




\subsubsection{Geschwindigkeiten}

\section{Ergebnisse der Geschwindigkeitsmessungen}

Die mittlere gemessene Geschwindigkeit des Radverkehrs betrug bei freier Fahrt $18,6 \mathrm{~km} / \mathrm{h}$ und bei freier regelkonformer Fahrt 18,7 km/h (Bild 7). Diese lag mehr als 2 km/h über dem von Schopf 1992 beschriebenen Wert von $16 \mathrm{~km} / \mathrm{h}$ und etwa 1,5 km/h höher als die von Falkenberg (2003) gemessenen $16,9 \mathrm{~km} / \mathrm{h}$.

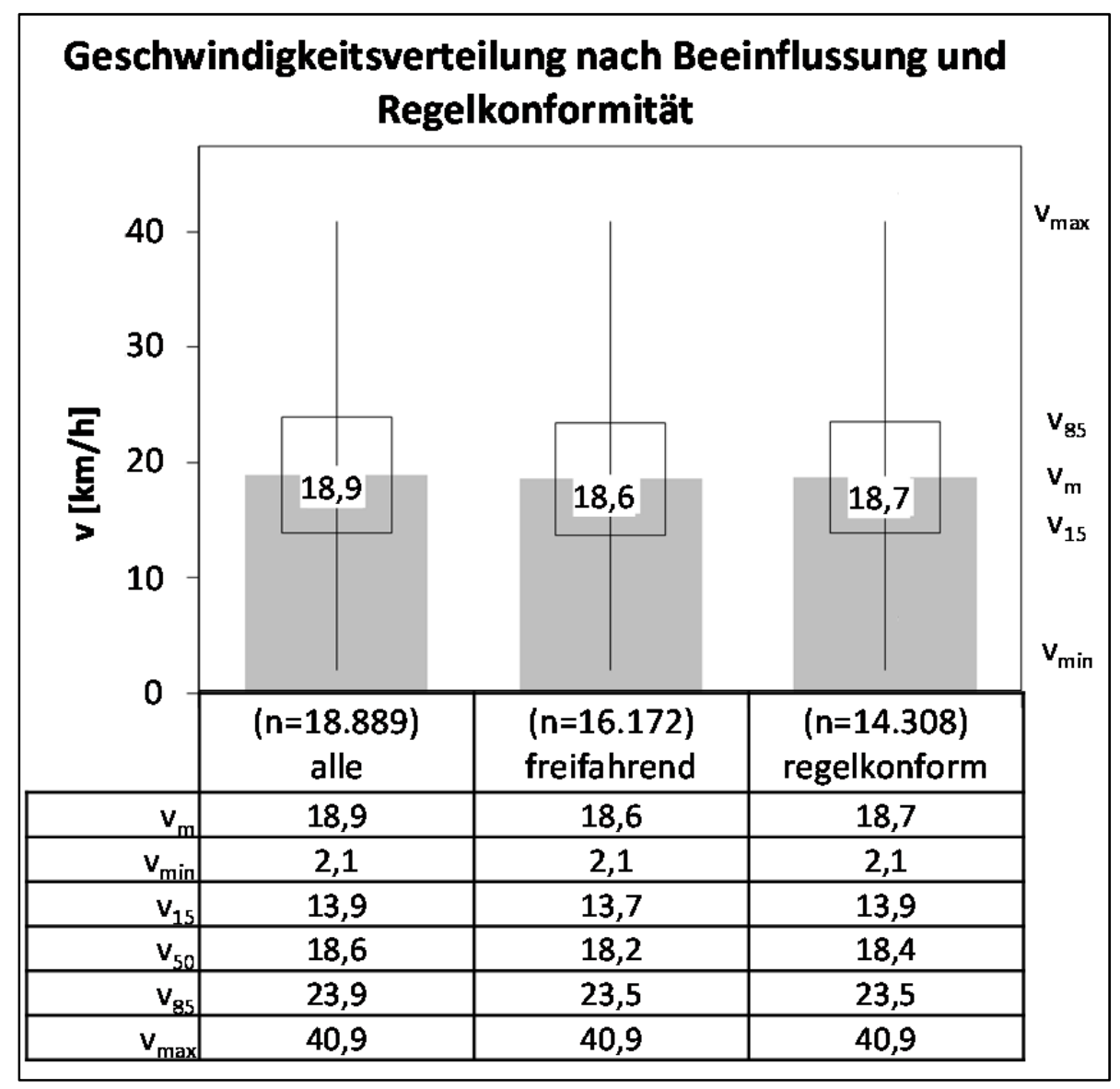

Bild 7: $\quad$ gemessene Geschwindigkeiten des Radverkehrs (mittlere Geschwindigkeit $v_{m}, 15 \%$-Quantil $v_{15}, 50 \%$-Quantil v50 und $85 \%$-Quantil $\mathbf{v}_{85}$ sowie Minimum $v_{\min }$ und Maximum $v_{\max }$

Die mittlere Geschwindigkeit von mit Cityrädern (Standardfahrrad) fahrenden Personen lag mit $18,3 \mathrm{~km} / \mathrm{h}$ im Bereich des Mittelwertes aller Radfahrer. Fahrer von Mountainbikes und insbesondere Rennräder fuhren dagegen schneller. Jeder zweite Rennradfahrer erreichte mindestens eine Geschwindigkeit von $24 \mathrm{~km} / \mathrm{h}$ und der Wert der $\mathrm{V}_{85}$ lag bei $30,3 \mathrm{~km} / \mathrm{h}$ (Bild 8). Mountain-Bike- und Rennradfahrer fuhren signifikant schneller als Nutzer von Standardfahrrädern, Radfahrer mit HollandRädern dagegen signifikant langsamer. (Bild 8 und Tabelle 13). Bei Standardrädern, Mountain-Bikes und Rennrädern wurden Maximalgeschwindigkeiten von etwa 40 km/h gemessen. 


\begin{tabular}{|r|r|r|c|c|c|}
\hline \multicolumn{6}{|c|}{ Geschwindigkeitsverteilung nach Fahrradtyp } \\
\hline 50 \\
40 \\
35 \\
30
\end{tabular}

Bild 8: Geschwindigkeiten und Fahrradtyp

Tabelle 13: Signifikanztest: Geschwindigkeiten und Fahrradtypen ${ }^{15}$

\begin{tabular}{|l|c|c|c|c|c|c|}
\hline \multicolumn{9}{|c|}{ berechnete t-Werte } \\
\hline & City/Stadt/Standardrad & Mountainbike & Rennrad & Lastenrad & Hollandrad & Pedelec 25 \\
\hline City/Stadt/Standardrad & & 2,578 & 2,584 & 2,654 & 2,580 & 2,629 \\
\hline Mountainbike & $\mathbf{- 1 7 , 6 0 3}$ & & 2,581 & 2,646 & 2,578 & 2,622 \\
\hline Rennrad & $\mathbf{- 2 6 , 7 4 9}$ & $\mathbf{- 1 5 , 5 5 2}$ & & 2,629 & 2,581 & 2,609 \\
\hline Lastenrad & 1,455 & $\mathbf{5 , 9 1 7}$ & $\mathbf{1 3 , 0 3 8}$ & & 2,644 & 2,601 \\
\hline Hollandrad & $-0,476$ & $\mathbf{4 , 4 4 2}$ & $\mathbf{1 2 , 1 9 1}$ & 1,298 & & 2,621 \\
\hline Pedelec 25 & $\mathbf{9 , 5 8 1}$ & $\mathbf{1 9 , 9 6 5}$ & $\mathbf{2 8 , 5 8 3}$ & 1,366 & $\mathbf{3 , 4 2 0}$ & \\
\hline
\end{tabular}

Die mittleren Geschwindigkeiten der augenscheinlich 18- bis 24-jährigen Radfahrer lagen etwa $1,5 \mathrm{~km} / \mathrm{h}$ über denen der 25 bis 65-Jährigen und etwa $1 \mathrm{~km} / \mathrm{h}$ über denen der augenscheinlich 15-bis 17-Jährigen.

Die fett markierten Felder heben Konstellationen hervor, welche sich laut t-Test signifikant unterscheiden. Signifikante Unterschiede bestehen dann, wenn der Betrag des berechneten t-Werts (Werte unterhalb der Hauptdiagonalen) größer als der Betrag des zugehörigen kritischen t-Werts (Werte oberhalb der Hauptdiagonalen) ist. Die kritischen t-Werte wurden aufgrund des mehrfach durchgeführten t-Tests über die Bonferroni-Anpassung der zugelassenen Irrtumswahrscheinlichkeit angepasst, sodass die kumulierte Gesamtirrtumswahrscheinlichkeit bei 5 \% lag. 
Die Altersgruppe "25-65 Jahre" wurde in einer weiteren Stichprobe in zwei weitere Untergruppen „25-49 Jahre“ und „50-65 Jahre“ aufgeteilt. Es zeigte sich, dass die Altersgruppe „18-24 Jahre“ und die Untergruppe „25-49 Jahre“ gleiche mittlere Geschwindigkeiten (Bild 9) aufwiesen

Kinder unter 14 und Über-65-Jährige fuhren mit deutlich geringeren Geschwindigkeiten. Die mittlere Geschwindigkeit der Über-65-Jährigen lag ca. 3 km/h unter dem Wert der 50- bis 65-Jährigen (Bild 9).

Für fast alle Altersgruppen waren die Unterschiede zwischen den Geschwindigkeiten statistisch signifikant (Tabelle 14). Zwischen den Kindern unter 10 Jahren und den Über-65-Jährigen konnte kein signifikanter Unterschied der Geschwindigkeiten festgestellt werden. Ebenso unterschieden sich die Geschwindigkeiten der Altersgruppen „18-24 Jahre“ und der Untergruppe „25-49 Jahre“ nicht signifikant (Tabelle 14).

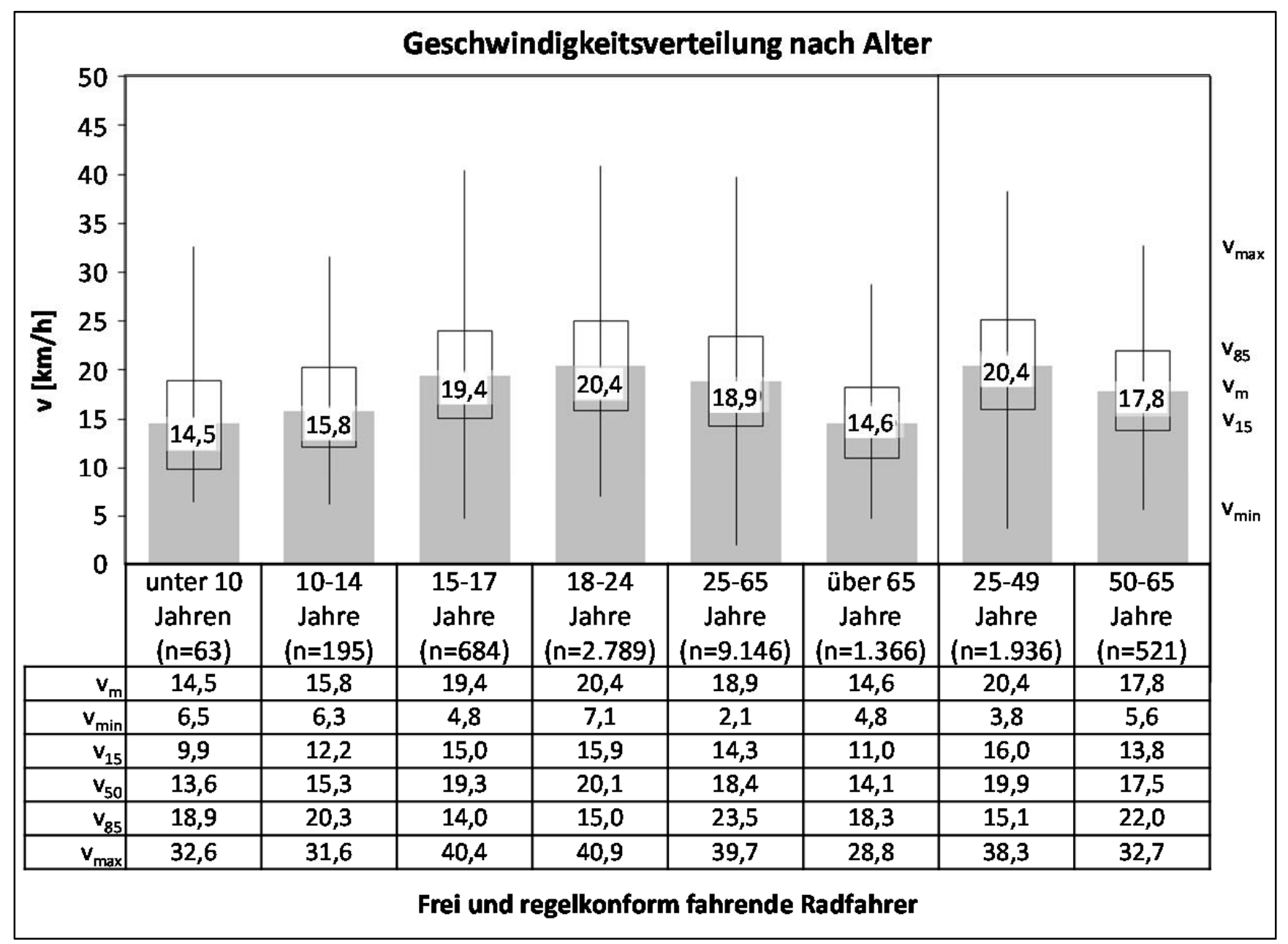

Bild 9: Geschwindigkeiten und Altersgruppe

Tabelle 14: Signifikanztest: Geschwindigkeiten nach Altersgruppen ${ }^{15}$

\begin{tabular}{|c|c|c|c|c|c|c|c|}
\hline \multicolumn{8}{|c|}{ berechnete $t$-Werte } \\
\hline & $<10$ Jahre & 10-14 Jahre & 15-17 Jahre & 18-24 Jahre & 25-49 Jahre & 50-65 Jahre & $>65$ Jahre \\
\hline$<10$ Jahre & & 2,633 & 2,646 & 2,654 & 2,654 & 2,646 & 2,653 \\
\hline 10-14 Jahre & $-1,850$ & & 2,590 & 2,597 & 2,596 & 2,589 & 2,596 \\
\hline 15-17 Jahre & $-7,472$ & $-10,702$ & & 2,581 & 2,580 & 2,580 & 2,580 \\
\hline 18-24 Jahre & $-9,224$ & $-15,342$ & $-4,856$ & & 2,577 & 2,582 & 2,558 \\
\hline 25-49 Jahre & $-9,157$ & $-15,001$ & $-4,574$ & 0,154 & & 2,581 & 2,578 \\
\hline 50-65 Jahre & 4,942 & $-5,775$ & 6,420 & 12,897 & 12,306 & & 2,582 \\
\hline > 65 Jahre & $-0,119$ & 3,979 & 23,470 & 43,897 & 40,069 & 15,168 & \\
\hline
\end{tabular}


Auf den fahrbahnseitigen Radverkehrsführungen (Mischverkehr, Radfahr- und Schutzstreifen sowie Bussonderstreifen) wurden tendenziell höhere Geschwindigkeiten gemessen als auf Radwegen (Bild $10)^{16}$. Die Unterschiede zwischen den Geschwindigkeiten waren für fast alle Radverkehrsführungen statistisch signifikant (Tabelle 15).

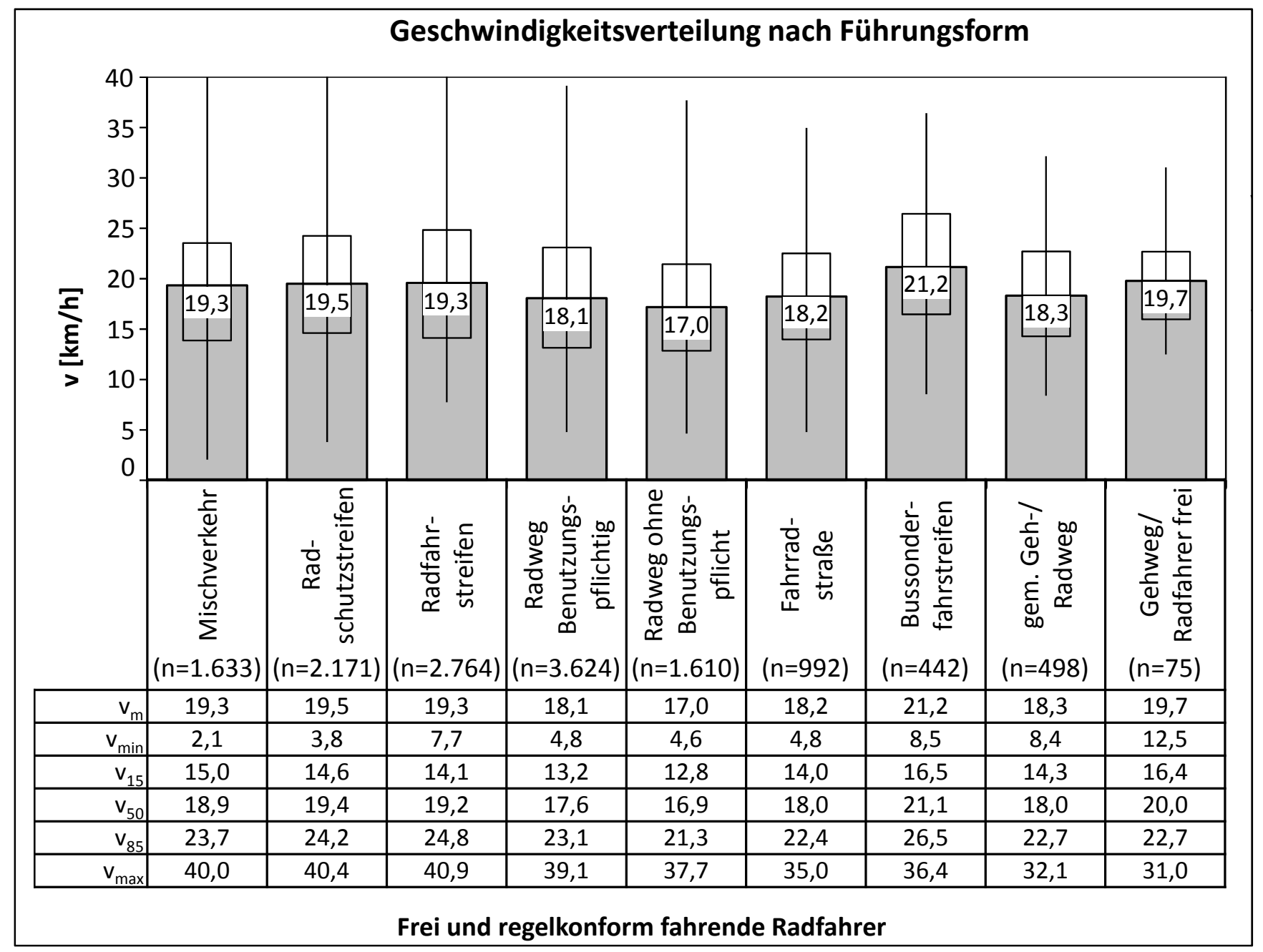

Bild 10:

Geschwindigkeit und Radverkehrsführung 
Tabelle 15: Signifikanztest: Geschwindigkeiten nach Radverkehrsführung ${ }^{15}$

\begin{tabular}{|c|c|c|c|c|c|c|c|c|c|}
\hline \multicolumn{10}{|c|}{ berechnete und kritische $\mathrm{t}$-Werte } \\
\hline & $\begin{array}{l}\text { Mischver- } \\
\text { kehr }\end{array}$ & $\begin{array}{c}\text { Radschutz- } \\
\text { schutz- } \\
\text { streifen }\end{array}$ & $\begin{array}{c}\text { Radfahrstrei- } \\
\text { fen }\end{array}$ & \begin{tabular}{|c|} 
Radweg \\
benutzungs- \\
pflichtig
\end{tabular} & $\begin{array}{c}\text { Radweg ohne } \\
\text { Benutzungs- } \\
\text { pflicht }\end{array}$ & $\begin{array}{l}\text { Fahrrad- } \\
\text { straße }\end{array}$ & $\begin{array}{l}\text { Bussonder- } \\
\text { fahrstreifen }\end{array}$ & $\begin{array}{l}\text { gem. Geh-I } \\
\text { Radweg }\end{array}$ & $\begin{array}{c}\text { Gehwegl } \\
\text { Radfahrer } \\
\text { frei }\end{array}$ \\
\hline Mischverkehr & & 3,092 & 3,092 & 3,093 & 3,093 & 3,093 & 3,102 & 3,10 & 3,19 \\
\hline Radschutzstreifen & 1,020 & & 3,092 & 3,092 & 3,094 & 3,093 & 3,101 & 3,099 & 3,189 \\
\hline Radfahrstreifen & 1,632 & 0,642 & & 3,092 & 3,092 & 3,092 & 3,103 & 3,100 & 3,189 \\
\hline Radweg & $-9,262$ & $-11,21$ & $-12,096$ & & 3,092 & 3,093 & 3,104 & 3,101 & 3,193 \\
\hline Radweg o.B. & $-14,13$ & 16,119 & 16,952 & 6,718 & & 3,093 & 3,103 & 3,099 & 3,188 \\
\hline Fahrradstraße & 3,992 & 8,431 & 9,13 & $-1,235$ & $-6,877$ & & 3,102 & 3,099 & 3,185 \\
\hline Bussonderfahrstreifen & $-7,36$ & $-6,893$ & 6,548 & $-13,300$ & $-16,449$ & $-11,909$ & & 3,100 & 3,157 \\
\hline gem. Geh-/Radweg & 4,986 & 5,932 & 6,425 & $-1,969$ & $-5,678$ & $-0,434$ & $-10,206$ & & 3,170 \\
\hline Gehweg/ Radfahrer frei & 4,18 & 0,683 & 0,469 & 4,18 & 6,232 & 3,700 & $-2,984$ & 3,332 & \\
\hline
\end{tabular}

\section{Maßgebliche Einflüsse auf die Geschwindigkeiten}

Im künftigen Radverkehr können sich die Geschwindigkeiten durch eine veränderte Altersstruktur von Radfahrern und veränderte Anteile der genutzten Fahrradtypen verändern. Es wurde daher untersucht, inwieweit die Altersstruktur, die Fahrradtypen oder die genutzten Anlagen die Geschwindigkeiten des Radverkehrs maßgebend beeinflussen. Hierzu wurden zunächst die mittleren Geschwindigkeiten frei und regelkonform fahrender Radfahrer verschiedenen Alters mit unterschiedlichen Fahrradtypen auf den unterschiedlichen Anlagen berechnet. Auf dieser Grundlage wurden dann die Geschwindigkeitsdifferenzen mit jeweils zwei konstant gehaltenen Variablen und einer unabhängigen Variable ermittelt (Kap. 7.7 im Anhang).

Wurden die Altersgruppen und Anlagentypen konstant gehalten und der Fahrradtyp als unabhängige Variable herangezogen, wichen die Geschwindigkeiten durchschnittlich um 1,0 km/h ab. Eine mit $1,2 \mathrm{~km} / \mathrm{h}$ etwas höhere Abweichung ergab sich für die Altersgruppe als unabhängige Variable (Tabelle 16). Gegenüber dem Anlagentyp und dem Fahrradtyp übte die Altersgruppe damit einen um $0,2 \mathrm{~km} / \mathrm{h}$ stärkeren Einfluss auf die Geschwindigkeiten im Radverkehr aus.

Tabelle 16: mittlere Abweichung der Geschwindigkeiten nach konstant gehaltenen und unabhängigen Variablen

\begin{tabular}{|l|c|c|c|}
\hline \multirow{2}{*}{\multicolumn{1}{|c|}{ Konstant gehaltene Variablen }} & mittlere Abweichung der Geschwindigkeiten [km/h] & \multicolumn{2}{c|}{ unabhängige Variable } \\
\cline { 2 - 4 } & Anlagentyp & - & \multicolumn{2}{c|}{ Fahrradtyp } \\
\hline Altersgruppe und Fahrradtyp & 1,0 & - & - \\
\hline Altersgruppe und Anlagentyp & - & - & - \\
\hline Anlagentyp und Fahrradtyp & - & 1,0 \\
\hline
\end{tabular}




\section{Verallgemeinerung der Untersuchungsergebnisse}

Die Auswertung der ermittelten Geschwindigkeitsdaten freifahrender Radfahrer führten zu einer mittleren Geschwindigkeit von 18,6 km/h. Die Anteile der verschiedenen Altersgruppen in der Stichprobe der Geschwindigkeitsmessungen wichen jedoch von den Anteilen der Altersgruppen an der Fahrleistung mit dem Fahrrad nach MiD 2008 ab. Um das Ergebnis der Messungen für Mittel- und Großstädte verallgemeinern zu können, wurde ergänzend die mittlere Geschwindigkeit der freifahrenden Radfahrer entsprechend den Verkehrsleistungen der Altersgruppen gewichtet berechnet. ${ }^{17}$ Die mittlere Geschwindigkeit des Radverkehrs hat sich demnach gegenüber den früheren Untersuchungen von Schopf (1992) und Falkenberg (2003) um etwa $1,5 \mathrm{~km} / \mathrm{h}$ erhöht und liegt nun bei $18,2 \mathrm{~km} / \mathrm{h}$. Die $v_{85}$ liegt bei $22,7 \mathrm{~km} / \mathrm{h}$. 


\subsubsection{Fahrverhalten an lichtsignalgeregelten Knotenpunkten}

Bei den Verhaltensbeobachtungen an den Knotenpunkten wurden von insgesamt 4.177 Radfahrern die Fahrlinien über den Knotenpunkt erfasst. Insbesondere die Fahrlinien der 883 linksabbiegenden Radfahrer waren von Interesse. Die Fahrlinien wurden getrennt erhoben nach den Radverkehrsanlagen in der beobachteten Zufahrt. Da sich in Abhängigkeit der Absetzung des Radweges von der Fahrbahn Unterschiede im Fahrverhalten zeigten, wurde das Fahrverhalten an Radwegen zusätzlich getrennt nach der Absetzung des Radweges ausgewertet.

Die Auswertung der Fahrlinien zeigte, dass Radfahrstreifen, Schutzstreifen als auch die Führung des Radverkehrs auf der Fahrbahn das direkte Linksabbiegen begünstigten. Radwege begünstigen die regelwidrige Nutzung linker Furten und die anschließende Fortführung der Fahrt auf der linken Straßenseite (Tabelle 17).

Tabelle 17: Häufige Fahrlinien linksabbiegender Radfahrer nach Radverkehrsführung

\begin{tabular}{|c|c|c|c|c|c|c|c|}
\hline Linksabbieger & Alle KP & Radweg & Radweg (0 m) & Radweg (>0 m) & Radfahrstreifen & Schutzstreifen & Fahrbahn \\
\hline$N=$ & 883 & 344 & 136 & 208 & 246 & 120 & 137 \\
\hline & $19 \%$ & $8 \%$ & $16 \%$ & $2 \%$ & $31 \%$ & $29 \%$ & $22 \%$ \\
\hline & $15 \%$ & $25 \%$ & $19 \%$ & $29 \%$ & $4 \%$ & $3 \%$ & $9 \%$ \\
\hline Sonstige & $66 \%$ & $67 \%$ & $65 \%$ & $69 \%$ & $65 \%$ & $68 \%$ & $69 \%$ \\
\hline
\end{tabular}

Von der Fahrbahn abgesetzte Radwegfurten unterstützen ein indirektes Linksabbiegen. Nicht abgesetzte Radwege begünstigten hingegen das direkte Linksabbiegen auf der Fahrbahn sowie die mehrfache regelwidrige Nutzung linker Furten (Tabelle 18).

Tabelle 18: Häufige Fahrlinien linksabbiegender Radfahrer nach Furtabsatzung bei Radwegen

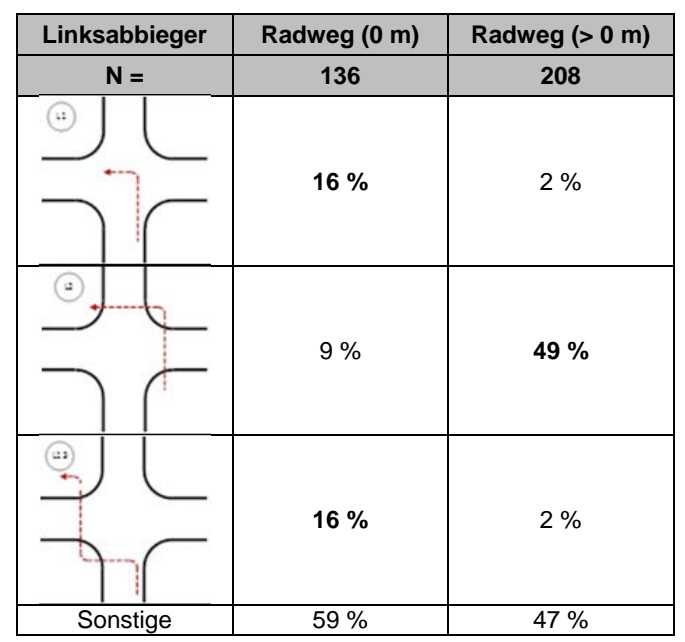


Bei der Führung des Radverkehrs auf der Fahrbahn wurde in der Tendenz das direkte Linksabbiegen bevorzugt. Fast ein Fünftel der beobachteten Radfahrer wichen aber auch in den Seitenraum aus und bogen hier über die Gehwegfurten links ab (Tabelle 19).

Tabelle 19: Häufige Fahrlinien linksabbiegender Radfahrer bei Fahrbahnführung

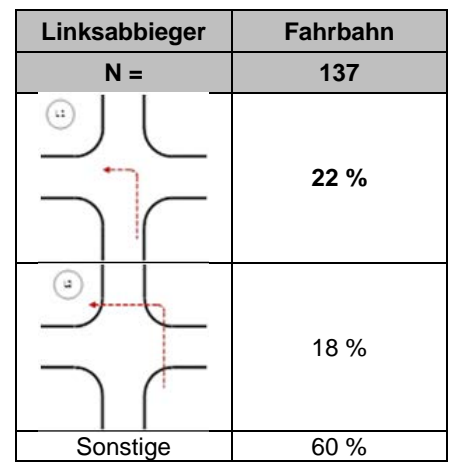

Bezüglich der geradeausfahrenden Radfahrer zeigte sich, dass an Radwegen ohne Furtabsetzung häufiger der Knotenpunkt auf der Fahrbahn gequert wurde (Tabelle 27).

Tabelle 20: Häufige Fahrlinien geradeausfahrender Radfahrer nach Furtabsetzung bei Radwegen

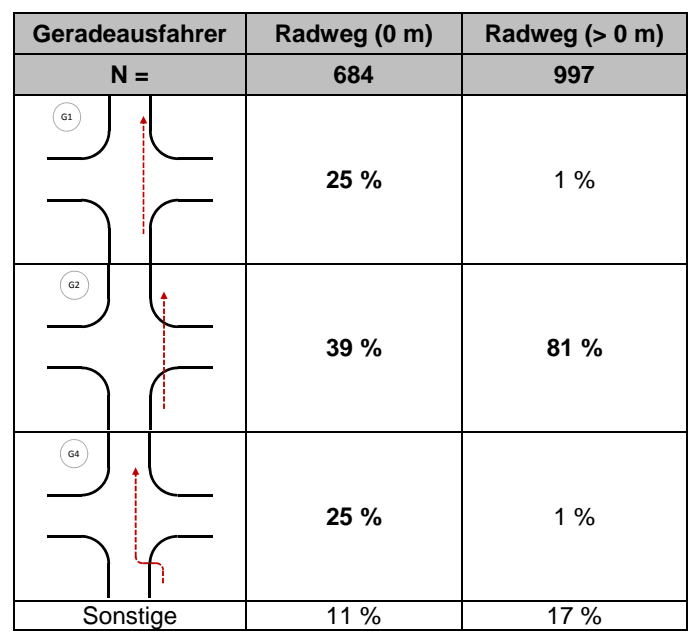

Eine vollständige Auflistung der Fahrlinien sowohl geradeausfahrender als auch links- und rechtsabbiegender Radfahrer nach unterschiedlichen Radverkehrsanlagentypen in den Zufahrten befindet sich im Anhang 7.8 


\subsubsection{Tragen eines Fahrradhelms}

In der Verkehrserhebung wurde auf den Untersuchungsstrecken das Tragen eines Helms erfasst. Im Mittel lag der Helmtrageanteil der Radfahrer bei ca. 15,6\%. In Abhängigkeit weiterer erhobener Merkmale (Erhebungsort, Fahrradtyp, Altersgruppe, Geschlecht), konnte der Anteil helmtragender Radfahrer differenzierter analysiert werden.

Die höchste Helmtragequote in den Untersuchungsstädten konnte in Dresden mit $29 \%$ festgestellt werden. Im Vergleich zu allen anderen Untersuchungsstädten war dies ein substantieller Unterschied. In den weiteren Untersuchungsstädten bewegte sich der Anteil helmtragender Radfahrer zwischen 9 und $18 \%$, wobei sich darunter keine weiteren substantiellen Unterschiede zeigten (Bild 11, Tabelle 21).

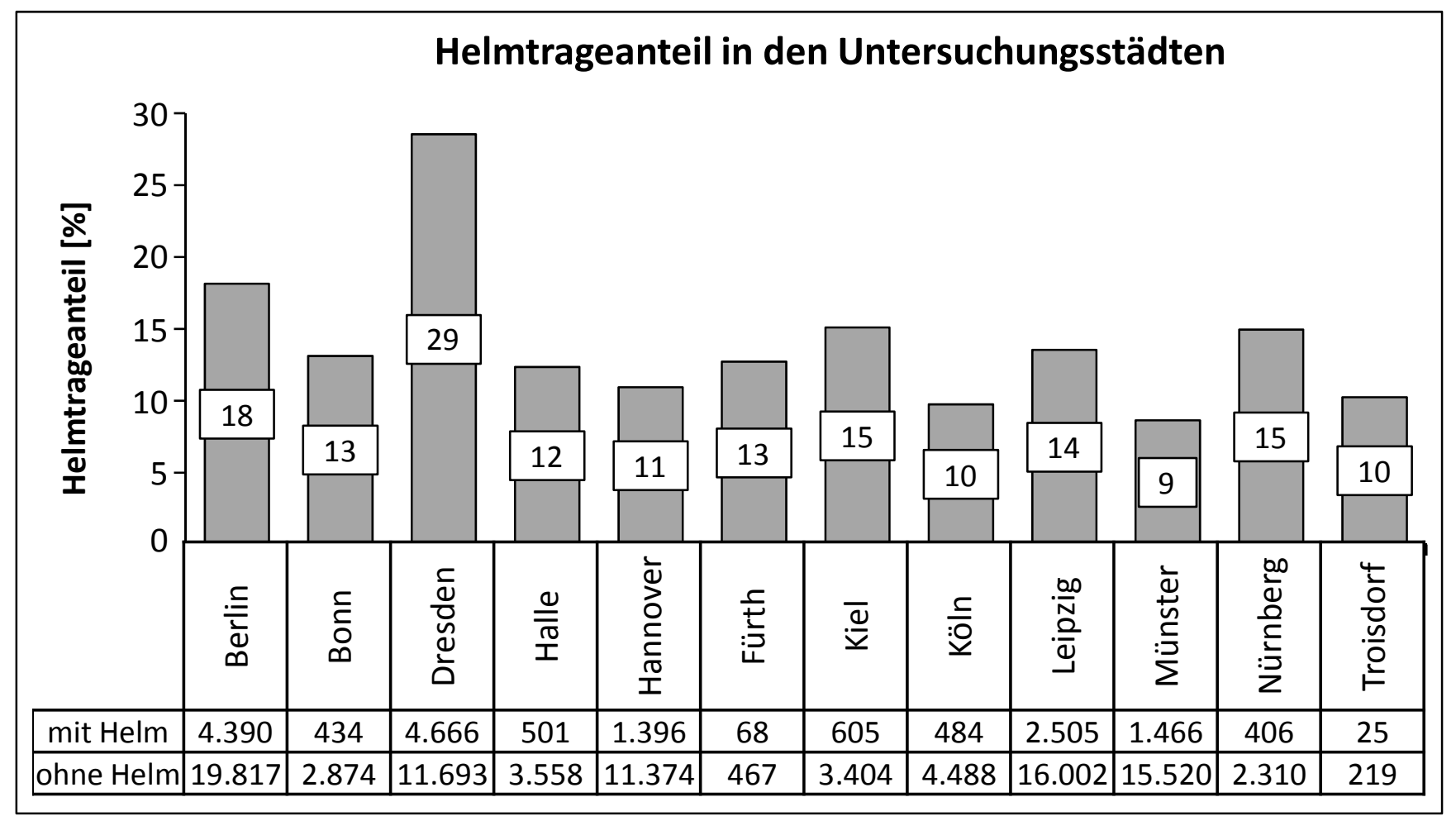

Bild 11: Erhebungsort und Helmtragequote 
Tabelle 21: Signifikanztest: Helmtragequote nach Erhebungsort ${ }^{18}$

\begin{tabular}{|l|c|c|c|c|c|c|c|c|c|c|c|}
\hline \multicolumn{9}{|c|}{ Berechneter normierter Kontingenzkoeffizient $\boldsymbol{\Phi}$} \\
\hline & Berlin & Bonn & Dresden & Halle & Hannover & Fürth & Kiel & Köln & Leipzig & Münster & Nürnberg \\
\hline Berlin & & & & & & & & & & & \\
\hline Bonn & 0,043 & & & & & & & & & & \\
\hline Dresden & $\mathbf{0 , 1 2 2}$ & $\mathbf{0 , 1 3 1}$ & & & & & & & & & \\
\hline Halle & 0,054 & 0,012 & $\mathbf{0 , 1 4 9}$ & & & & & & & & \\
\hline Hannover & 0,094 & 0,028 & $\mathbf{0 , 2 1 5}$ & 0,019 & & & & & & & \\
\hline Fürth & 0,020 & 0,060 & 0,061 & 0,004 & 0,011 & & & & & & \\
\hline Kiel & 0,028 & 0,004 & $\mathbf{0 , 1 2 2}$ & 0,040 & 0,055 & 0,022 & & & & & \\
\hline Köln & 0,085 & 0,053 & $\mathbf{0 , 1 8 6}$ & 0,042 & 0,017 & 0,029 & 0,082 & & & & \\
\hline Leipzig & 0,062 & 0,004 & $\mathbf{0 , 1 8 5}$ & 0,013 & 0,039 & 0,004 & 0,017 & 0,047 & & & \\
\hline Münster & $\mathbf{0 , 1 3 4}$ & 0,057 & $\mathbf{0 , 2 5 7}$ & 0,050 & 0,039 & 0,025 & 0,085 & 0,016 & 0,078 & & \\
\hline Nürnberg & 0,025 & 0,026 & $\mathbf{0 , 1 0 7}$ & 0,037 & 0,048 & 0,024 & 0,002 & 0,078 & 0,014 & 0,074 & \\
\hline Troisdorf & 0,020 & 0,022 & 0,049 & 0,015 & 0,003 & 0,035 & 0,030 & 0,036 & 0,011 & 0,007 & 0,040 \\
\hline
\end{tabular}

Die höchste Helmtragequote von $28 \%$ konnte für Fahrer von Rennrädern festgestellt werden. Mit 19 \% lagen auch die Fahrer von Mountainbikes über dem Durchschnitt. Die Helmtragequote der Fahrer von Lastenrädern zeigte einen substantiellen Unterschied zu der Quote von Fahrern von Rennrädern und Mountainbikes (Bild 12, Tabelle 22).

Chi-Quadrat-Mehrfeldertests errechnen bei großen Kollektive häufig signifikante Unterschiede zwischen Merkmalen. Als statistisches Zusammenhangsmaß wurde daher der normierte Kontingenzkoeffizient $\Phi$ errechnet, welcher die Stärke des Zusammenhangs zwischen zwei Größen auf dem Intervall $[0,1]$ angibt. Ein Wert von „0“ ergibt sich bei dieser Anwendung, wenn sich keine unterschiedliche Ausprägungen der Helmtragequote differenziert für die einzelnen Merkmale (Erhebungsort, Fahrradtyp, Altersgruppe, Geschlecht) ergeben; „1“ bedeutet entsprechend, dass die Unterschiede der Helmtragequote einzig dem Merkmal zugeordnet werden können. Werte von $\Phi \geq 0,1$ können als substantieller Zusammenhang zwischen Merkmal und Helmtragequote interpretiert werden (Mullins, 1974). Die fett markierten Felder heben signifikant unterschiedliche Merkmale hervor. 


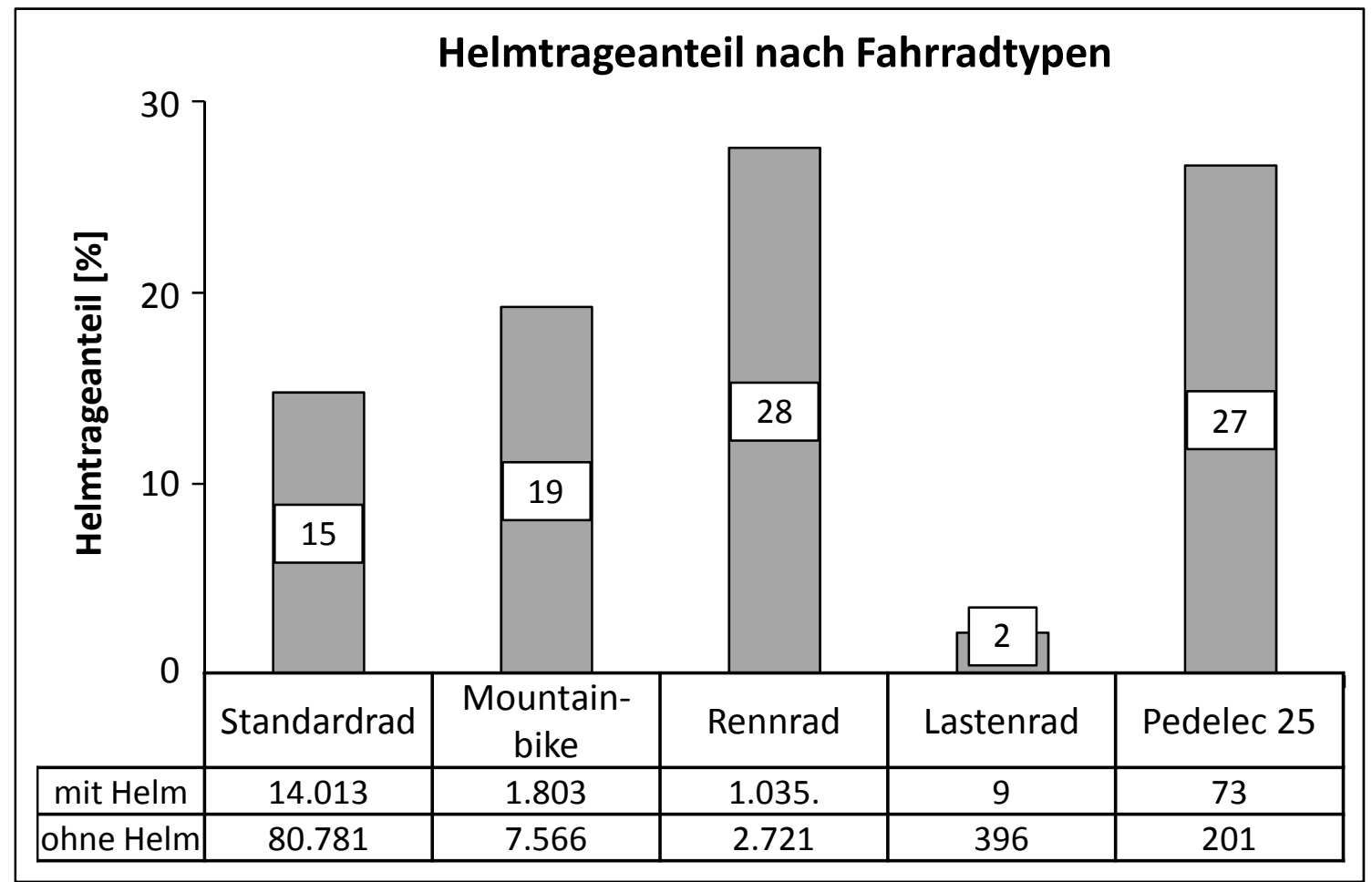

Bild 12: Fahrradtyp und Helmtragequote

Tabelle 22: Signifikanztest: Helmtragequote und Fahrradtypen ${ }^{18}$

\begin{tabular}{|l|c|c|c|c|c|}
\hline \multicolumn{5}{|c|}{ berechneter normierter Kontingenzkoeffizient $\boldsymbol{\Phi}$} & Pedelec $\mathbf{2 5}$ \\
\hline & City/Stadt/Standardrad & Mountainbike & Rennrad & & \\
\hline City/Stadt/Standardrad & & & & & \\
\hline Mountainbike & 0,036 & & & & \\
\hline Rennrad & 0,068 & 0,091 & & & \\
\hline Lastenrad & 0,023 & 0,087 & $\mathbf{0 , 1 7 3}$ & & \\
\hline Pedelec 25 & 0,018 & 0,031 & 0,005 & $\mathbf{0 , 3 6 8}$ & \\
\hline
\end{tabular}

Eine überdurchschnittlich hohe Helmtragequote konnte für Kinder unter 15 Jahren festgestellt werden. Dabei lag die Quote mit $70 \%$ bei Kindern unter 10 Jahren deutlich höher, als für Kinder zwischen 10 und 14 Jahren (45\%). Beide Altersgruppen zeigten substantielle Unterschiede zu den anderen Altersgruppen. Deutlich unterdurchschnittlich verhielt sich die Quote bei Radfahrern zwischen 15 und 24 Jahren. Als kollektivstärkste Altersgruppe lag die Helmtragequote der 25- bis 65-Jährigen nahe dem Durchschnitt (Bild 13, Tabelle 23). 


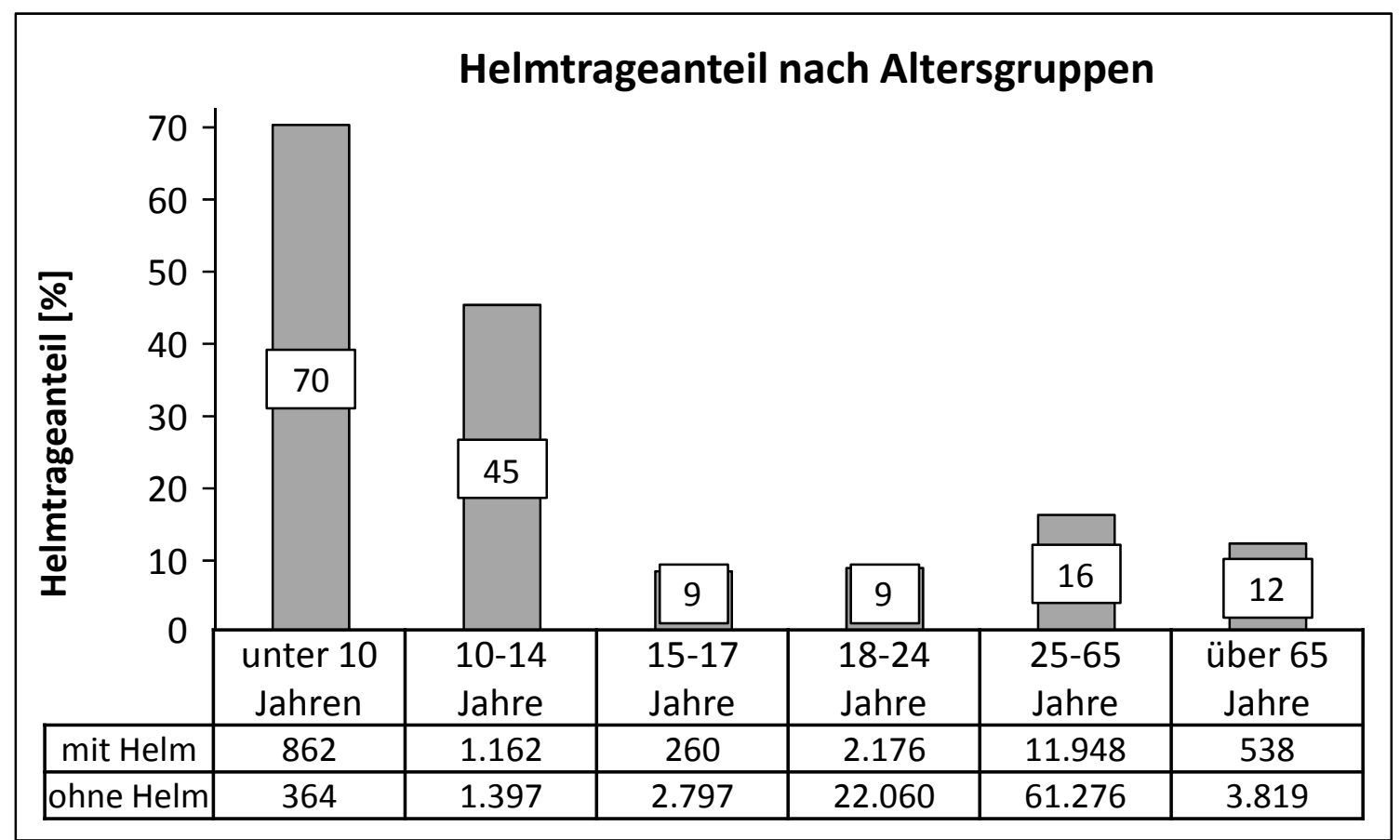

Bild 13: Altersgruppe und Helmtragequote

Tabelle 23: Signifikanztest: Helmtragequote und Altersgruppe ${ }^{18}$

\begin{tabular}{|l|c|c|c|c|c|c|}
\hline \multicolumn{7}{|c|}{ berechneter normierter Kontingenzkoeffizient $\boldsymbol{\Phi}$} \\
\hline & $<\mathbf{1 0}$ Jahre & $\mathbf{1 0 - 1 4}$ Jahre & $\mathbf{1 5 - 1 7}$ Jahre & $\mathbf{1 8 - 2 4}$ Jahre & 25-65 Jahre & $>$ 65 Jahre \\
\hline$<$ 10 Jahre & & & & & & \\
\hline 10-14 Jahre & $\mathbf{0 , 2 3 4}$ & & & & & \\
\hline 15-17 Jahre & $\mathbf{0 , 0 6 3 5}$ & $\mathbf{0 , 4 2 3}$ & & & & \\
\hline 18-24 Jahre & $\mathbf{0 , 4 0 5}$ & $\mathbf{0 , 3 2 4}$ & 0,005 & & & \\
\hline 25-65 Jahre & $\mathbf{0 , 1 8 2}$ & $\mathbf{0 , 1 3 9}$ & 0,042 & 0,090 & & \\
\hline$>$ 65 Jahre & $\mathbf{0 , 5 5 4}$ & $\mathbf{0 , 3 7 1}$ & 0,061 & 0,041 & 0,045 & \\
\hline
\end{tabular}


Männliche Radfahrer fuhren zu einem höheren Anteil mit Helm, als weibliche Radfahrer. Der Unterschied zwischen beiden war jedoch nicht substantiell (Bild 14, Tabelle 24).

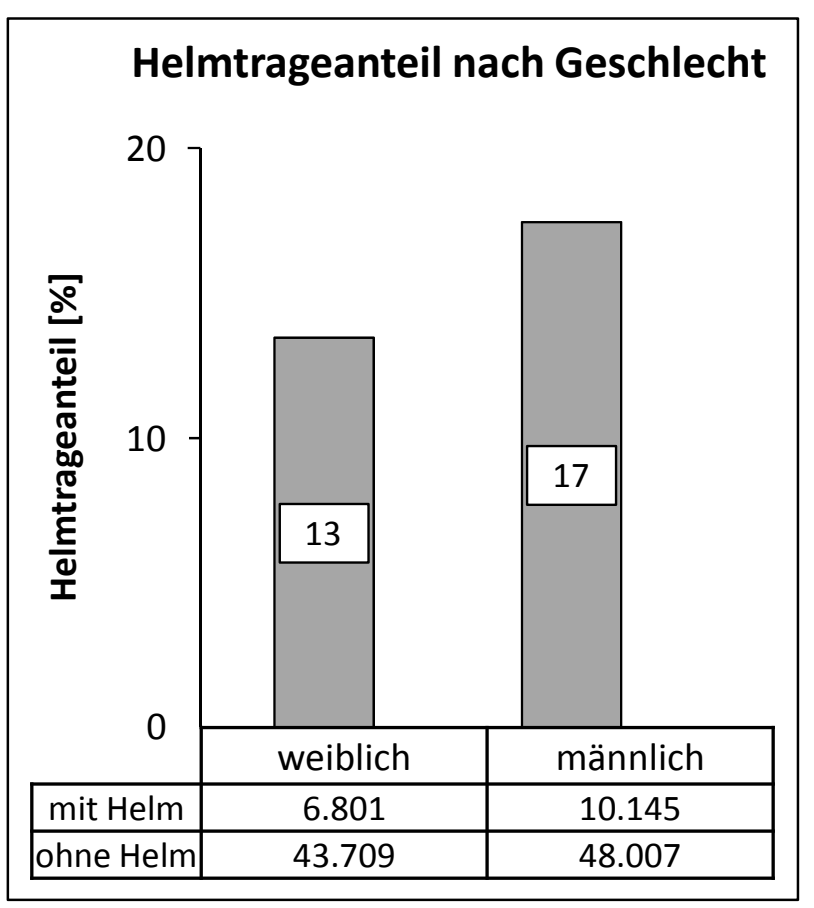

Bild 14: Geschlecht und Helmtragequote

Tabelle 24: Signifikanztest: Helmtragequote und Geschlecht ${ }^{18}$

\begin{tabular}{|l|c|c|}
\hline \multicolumn{3}{|c|}{ berechneter normierter Kontingenzkoeffizient $\Phi$} \\
\hline & weiblich & männlich \\
\hline weiblich & & \\
\hline männlich & 0,055 & \\
\hline
\end{tabular}




\subsubsection{Verteilung der Radfahrerunfälle im Straßenraum}

Auf Basis eines erweiterten Unfallkollektivs ${ }^{19}$ wurde die Aufteilung der Unfälle im Straßenraum aus den Angaben zur Charakteristik der Unfallstelle und zur Lichtzeichenanlage abgeleitet. Radfahrerunfälle konzentrierten annähernd zur Hälfte auf Knotenpunkte, wobei der Großteil der Unfälle mit $34 \%$ an Knotenpunkten ohne Lichtsignalanlage und $22 \%$ an lichtsignalgesteuerten Knotenpunkten stattfand. Ein Drittel der Unfälle fand auf Streckenabschnitten statt. Die verbleibenden Unfälle mit Radfahrerbeteiligung fanden an Zufahrten statt (Bild 15).

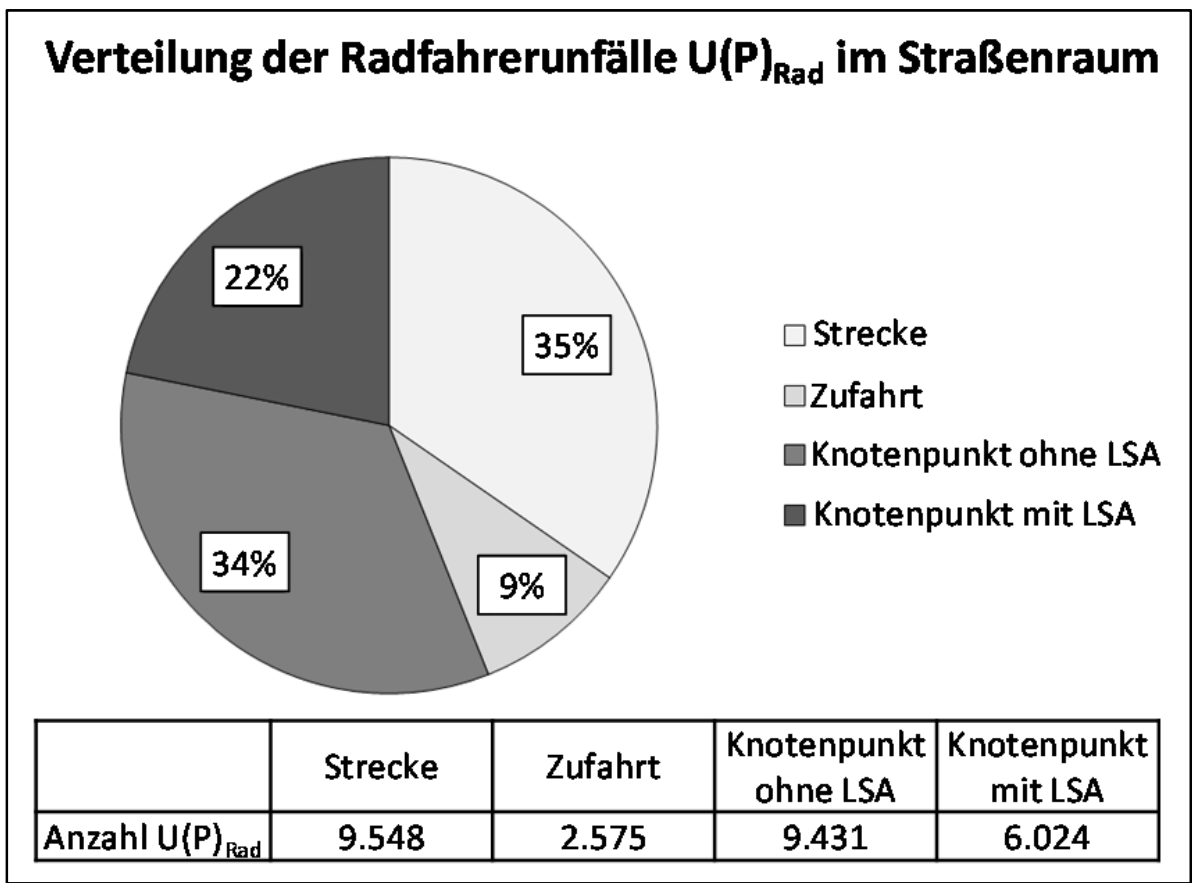

Bild 15: Verteilung der Radfahrerunfälle $U(P)_{\text {Rad }}$ im Straßenraum ${ }^{19}$ 


\subsubsection{Fazit}

Die mittleren Geschwindigkeiten des Radverkehrs haben sich gegenüber früheren Untersuchungen um etwa 1,5 km/h erhöht und liegen nun bei etwa 18,2 km/h. Mit Fahrrädern ohne elektromotorische Unterstützung fahren anteilig mehr Radfahrer im Geschwindigkeitsbereich 20-25 km/h als noch 1992 bzw. 2003.

Mit Mountain-Bikes und Rennrädern, die nach den Zählungen dieser Untersuchung und nach der Verkaufsstatistik etwa 12-15\% der Fahrräder stellen, fuhren $15 \%$ ihrer Nutzer schneller als 25 bzw. $29 \mathrm{~km} / \mathrm{h}$. Auch mit Standardfahrrädern fuhren $15 \%$ ihrer Nutzer schneller als $22 \mathrm{~km} / \mathrm{h}$. Gegenüber dem Radverkehrsanlagentyp und dem Fahrradtyp übt die Altersgruppe der Radfahrer einen größeren Einfluss auf die Geschwindigkeiten im Radverkehr aus.

Nach dem derzeitigen Kenntnisstand ist offen, ob die Geschwindigkeiten des Radverkehrs Auswirkungen auf die Verkehrssicherheit haben. Wirkungen der heutigen Radfahrergeschwindigkeiten auf die Unfallanzahl, die Unfallschwere und die Unfalltypen werden daher in Kapitel 4.4 untersucht.

An lichtsignalgeregelten Knotenpunkten zeigten sich Unterschiede im Abbiege- und KreuzenVerhalten der Radfahrer je nach Führungsform. Radfahrstreifen, Schutzstreifen und die Führung auf der Fahrbahn begünstigten das direkte Linksabbiegen. An Radwegen erfolgte dagegen häufig die regelwidrige Nutzung linker Furten, wobei von der Fahrbahn abgesetzte Radwege dazu beitragen können, ein indirektes Linksabbiegen zu unterstützen. Darüber hinaus war beim Kreuzen an abgesetzten Radwegen ein Wechsel auf die Fahrbahn seltener. Bei der Führung auf der Fahrbahn erfolgte das Linksabbiegen annähernd gleich häufig direkt als auch indirekt.

Die Analyse der Helmtragequote zeigte, dass zwei von drei Radfahrern unter 10 Jahren einen Helm tragen. Radfahrer zwischen 10 und 15 Jahren trugen etwa halb so oft einen Helm, lagen damit aber immer noch über dem Durchschnitt von 15,6\%. Auch Fahrer von Rennrädern und Mountainbikes zeigten substantiell höhere Helmtragequoten.

\subsection{Radverkehrsstärken, Unfallgeschehen und Regelbefolgung}

Vor dem Hintergrund bisheriger Erkenntnisse wird vermutet, dass die Radverkehrsstärke Einfluss auf das zukünftige Unfallgeschehen und Verkehrsverhalten von Radfahrern hat. Im Folgenden wurden daher Erkenntnisse aus den einzelnen Untersuchungsschritten zur Wirkung der Radverkehrsstärken auf die Unfallanzahl, die Unfallschwere, die Unfalltypenverteilung und die Regelbefolgung der Radfahrer zusammengefasst. Dabei wurde jeweils nach Streckenabschnitte und lichtsignalgeregelten Knotenpunkten differenziert. 


\subsubsection{Radverkehrsstärke und Unfallanzahl}

\section{Streckenabschnitte}

Grundlagen der Makroanalyse waren einerseits die Unfalldaten der 200 Streckenabschnitte und andererseits die vor Ort gezählten bzw. daraus auf den Tagesverkehr und die Jahresmengen hochgerechneten Radverkehrsstärken.

Um, je nach Führungsform, den Einfluss der Radverkehrsstärke auf die Unfallanzahl zu beschreiben, wurde die Gegenüberstellung getrennt nach den Führungsformen des Streckenkollektivs (Fahrbahn, Radfahrstreifen, Radweg und Schutzstreifen) vorgenommen. In Bild 16 sind die dreijährlichen Unfallzahlen auf den Streckenabschnitten (nicht längenbezogen) über den durchschnittlich-täglichen Verkehrsaufkommen im Radverkehr (hochgerechnet aus den Stichprobenzählungen) aufgetragen.

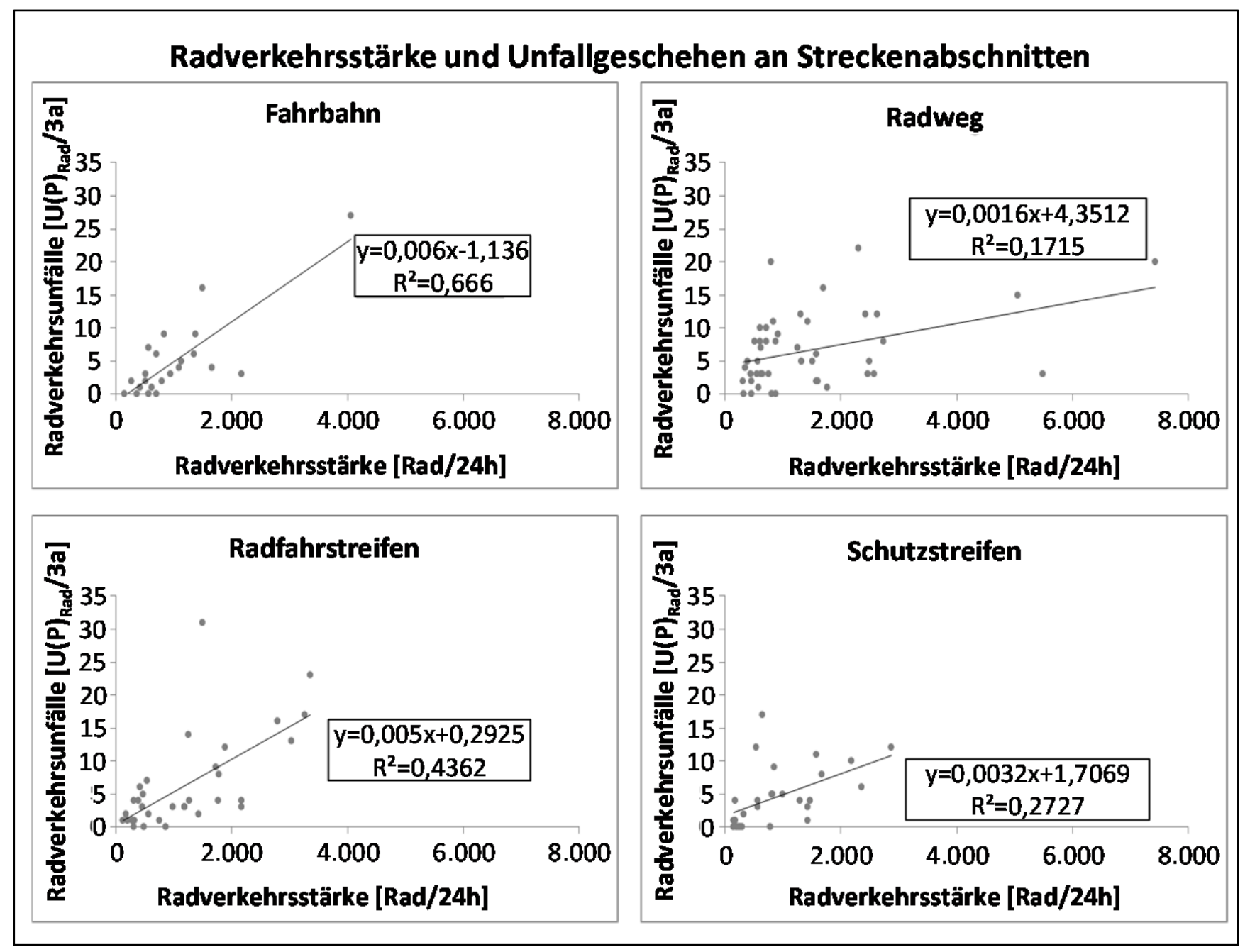

Bild 16: Unfallzahlen $U(P)_{\text {Rad }} / 3 a$, Verkehrsstärken Rad/24h und Führungsformen des Radverkehrs

Unter der vereinfachten Annahme eines linearen Zusammenhangs konnten für die vier Führungsformen mit der jeweils angegebenen Sicherheit (Bestimmtheitsmaß $R^{2}$ ) ein Zusammenhang zwischen 
beiden Größen erkannt werden (Bild 16) ${ }^{20}$. Der Verlauf der linearen Regressionsfunktionen zeigte, dass alle dargestellten Führungsformen zum einen unterschiedliche Unfallzahlen bei niedrigen Radverkehrsstärken und zum anderen unterschiedliche radverkehrsstärkenbedingte Unfallzunahmen (Anstieg der Regressionskurven) aufwiesen. So zeigte die Führung auf der Fahrbahn ein niedriges Unfallgeschehen bei geringer Belastung und einen starken Anstieg des Unfallgeschehens mit der Radverkehrsstärke. Dahingegen zeigte sich an Radwegen das höchste Ausgangsniveau im Unfallgeschehen bei einem geringen Anstieg der Radverkehrsunfälle mit der Radverkehrsstärke. Die Zusammenhänge waren allerdings für Radwege und Schutzstreifen sehr schwach ausgeprägt $\left(R^{2}<0,3\right)$, die Verläufe gelten auch nur für den mit empirischen Werten besetzten Bereich, denn bei sehr kleinen Verkehrsstärken müssen auch die Radunfallzahlen bei allen Führungsformen gegen Null tendieren.

Weitere Einflüsse z. B. aus der unterschiedlichen Streckenlänge oder der Umfeldnutzung wurden an dieser Stelle nicht berücksichtigt, wurden jedoch im Unfallmodell geprüft.

Die Basis der rechentechnischen Modellierung im Unfallmodell (beschrieben im Anhang 7.1) bildeten die Unfalldaten $\left(U(P)_{\text {Rad }}\right)$ der 200 Streckenabschnitte und die vor Ort erhobenen radverkehrsspezifischen (Verkehrsstärken, Geschwindigkeiten, Altersverteilung etc.) sowie streckenspezifischen (Streckenlängen, Umfeldnutzung etc.) Daten. Eine vollständige Auflistung der untersuchten Parameter befindet sich im Anhang 7.1.4.

Als systematisch wirksame Einflüsse auf das Unfallgeschehen haben sich im Unfallmodell die folgenden Größen gezeigt:

- Streckenlänge

- Radverkehrsstärke

- Führungsform

- Umfeldnutzung

- Anzahl der Anschlussknotenpunkte ${ }^{21}$ rungsformen nicht nachgewiesen werden

21 Streckenzüge wurden als Abschnitte zwischen zwei Verkehrsknotenpunkten definiert, Anschlussknotenpunkte waren damit Bestandteile von Streckenzügen 


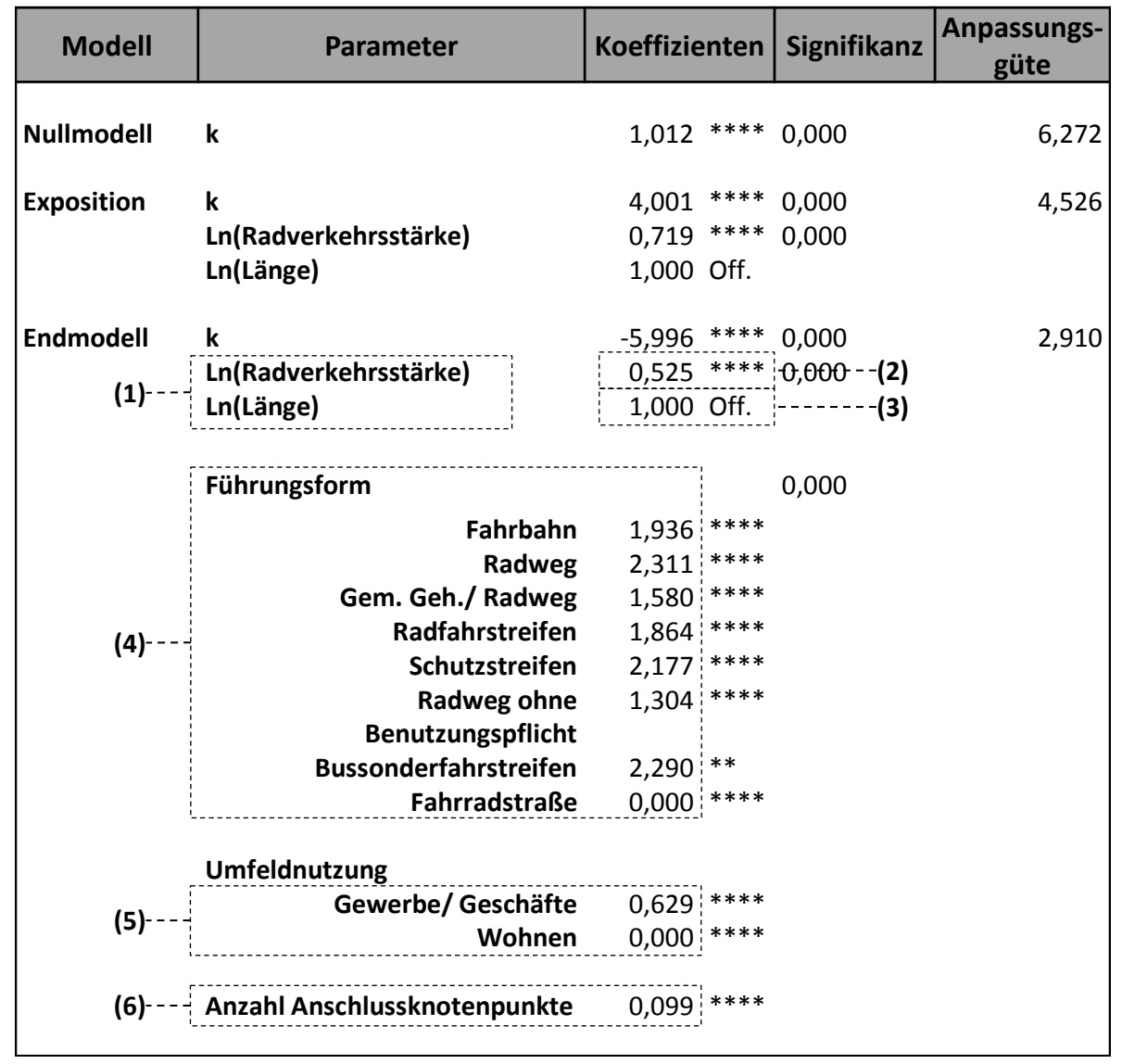

Bild 17: Modellparameter mit systematischem Einfluss auf die Radverkehrsunfallanzahl $U(P)_{\text {Rad }}$

Anhand der kalibrierten Parameter der einzelnen Einflussgrößen ließen sich die folgenden Erkenntnisse ziehen (Bild 17):

1. Die Radverkehrsstärke und die Streckenlänge gehen als Expositionsgrößen in das Modell ein.

2. Die Radverkehrsstärke hat einen degressiven Einfluss auf das Radunfallgeschehen, d. h. die Anzahl der Unfälle steigt in geringerem Maße als die Radverkehrsstärke an.

3. Der Streckenlänge wird aus sachlogischen Gründen ein linearer Einfluss auf die Anzahl der Unfälle unterstellt. Sie wurde deshalb als Offset-Größe ${ }^{22}$ in das Modell aufgenommen.

4. Die einzelnen Führungsformen haben ohne Verkehrsstärkeeinfluss ein unterschiedlich hohes Unfallgeschehen (siehe auch Bild 17).

5. An Gewerbe-/ Geschäftsstraßen ist bei sonst gleichen Bedingungen mit höheren Unfallzahlen $\left(U(P)_{\text {Rad }}\right)$ zu rechnen.

6. Mit zunehmender Anzahl der Anschlussknotenpunkte steigt die Anzahl der Unfälle auf den Streckenabschnitten.

22 Normierende Größe. Die Unfälle wurden normiert auf die Unfallanzahl je Streckenkilometer. Die Zielgröße im Modell war dadurch die Unfalldichte. Es wurde somit ein linearer Zusammenhang von Unfällen und Streckenlänge angenommen. 
Für Radwege und Schutzstreifen müssen verhältnismäßig hohe Unfalldichtenzuschläge angesetzt werden; für Fahrradstraßen hingegen ergeben sich keine weiteren Zuschläge. Dies entspricht den Erkenntnissen aus der eindimensionalen Regressionsanalyse (siehe Bild 17).

Anhand der jeweils ermittelten Anpassungsgüte ${ }^{23}$ ließ sich ableiten, wie groß der Teil der systematischen Abweichungen durch die im Modell enthaltenen Einflussgrößen beschrieben wurde. Ein Wert von „1“ entspricht dabei einer perfekten Anpassung an eine Poissonverteilung, d. h. die Einzelergebnisse werden nur noch durch die Zufallswahrscheinlichkeit beeinflusst. Ohne Berücksichtigung weiterer Einflüsse (Nullmodell) konnte die Gesamtstreuung über die Anpassungsgüte des Nullmodells $(6,272-1=5,272)$ abgeschätzt werden. Die Größe der verbleibenden unerklärten Streuung, nachdem die wirksamen Einflussgrößen in das Modell aufgenommen wurden (Endmodell), wird über die Anpassungsgüte des Endmodells abgeschätzt $(2,91-1=1,91)$. Der Anteil der erklärten Streuung ergab sich somit aus:

Formel 1:

$1-\frac{1,91}{5,272}=63,7 \%$

Ein systematischer Einfluss der Altersverteilung und der von Rad Fahrenden gefahrenen Geschwindigkeiten auf den erhobenen Strecken konnte im Hauptmodell rechnerisch nicht nachgewiesen werden. Da die gefahrenen Radverkehrsgeschwindigkeiten und die Radverkehrsstärke miteinander korrelierten, konnte dennoch ein Einfluss der Geschwindigkeit grundsätzlich nicht ausgeschlossen werden. In einem weiteren Untermodell (siehe 4.4.1) wurde deshalb der isolierte Einfluss der Geschwindigkeit ermittelt.

Bezüglich des Einflusses der Radverkehrsstärken kann zusammenfassend festgestellt werden, dass die Anzahl der Radverkehrsunfälle zwar grundsätzlich mit steigender Radverkehrsstärke zunimmt, diese Zunahme aber mit höherem Radverkehr geringer wird. Da neben der Radverkehrsstärke die Führungsformen spezifische Einflüsse ausüben, muss für jede Führungsform ein weiterer Zuschlagswert angesetzt werden.

Das entwickelte Modell mit seinen in Bild 17 aufgelisteten Modellparameter können als Formel wie folgt geschrieben werden:

Formel 2:

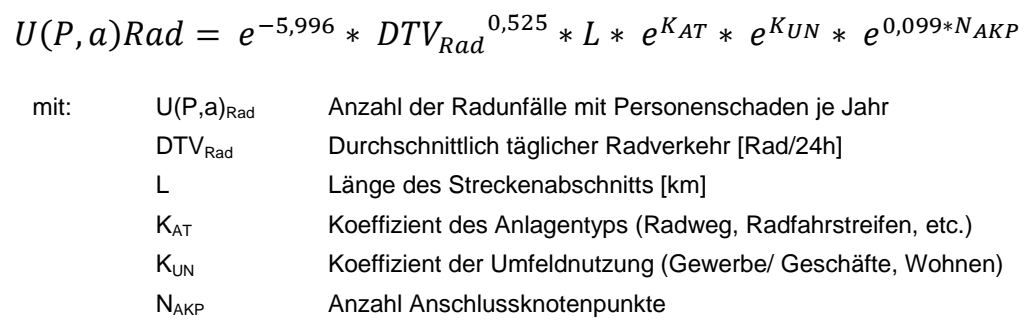


Die mikroskopische Unfallanalyse bestätigte, dass steigende Radverkehrsstärken auf den Anlagen auf höhere Unfallzahlen hinwirken. Bei fast allen Unfällen hatten die verunglückten Radfahrer die jeweiligen Radverkehrsanlagen genutzt (Tabelle 25).

Tabelle 25: Flächennutzung verunglückter Radfahrer (Anzahl U (P))

\begin{tabular}{|c|c|c|c|c|c|c|}
\hline \multirow{3}{*}{ Radverkehrsführung } & \multicolumn{6}{|c|}{ Fahrtrichtung } \\
\hline & \multicolumn{3}{|c|}{ regelwidrig links fahren auf } & \multicolumn{3}{|c|}{ rechts fahren auf } \\
\hline & $\begin{array}{l}\text { Radverkehrs- } \\
\text { anlage }\end{array}$ & Gehweg & Fahrbahn & $\begin{array}{c}\text { Radverkehrs- } \\
\text { anlage }\end{array}$ & Gehweg & Fahrbahn \\
\hline benutzungspflichtige Radwege & 66 & 6 & 2 & 103 & 5 & 3 \\
\hline nicht benutzungspflichtige Radwege & 5 & 1 & 0 & 3 & 0 & 0 \\
\hline Radfahrstreifen & 9 & 3 & 0 & 59 & 0 & 0 \\
\hline Schutzstreifen & 1 & 3 & 0 & 20 & 1 & 1 \\
\hline Fahrbahn & 0 & 5 & 0 & & 1 & 65 \\
\hline
\end{tabular}

\section{Unfallraten auf Streckenabschnitten}

Bei einem Vergleich der untersuchten Anlagentypen zeigte sich, dass Radwegführungen an Verkehrsstraßen gegenüber Radfahr- und Schutzstreifen und Mischverkehrsführungen leicht höhere Unfallraten aufwiesen, Fahrradstraßen demgegenüber aber erheblich niedrigere Unfallraten hatten (Bild $18)^{24}$. In den untersuchten Fahrradstraßen bestand dabei kein Zusammenhang zwischen den Radverkehrsstärken und der jeweiligen Unfallanzahl.

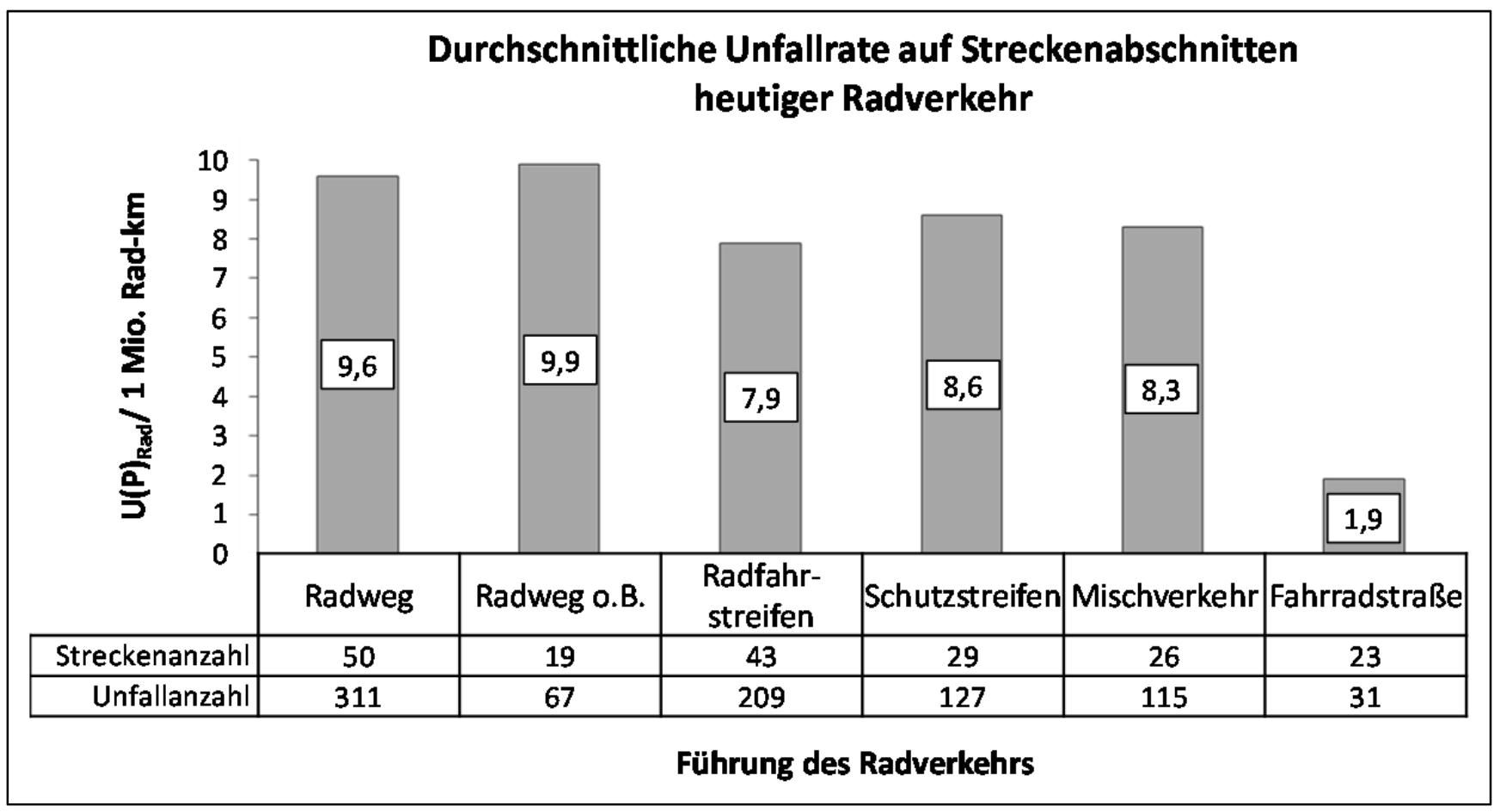

Bild 18:

Unfallraten des heutigen Radverkehrs nach Anlagentypen ( $\mathrm{n}=$ Anzahl Streckenabschnitte) 


\section{Lichtsignalgeregelte Knotenpunkte}

Aus der Makroanalyse lagen für die lichtsignalgeregelten Knotenpunkte keine Erkenntnisse vor, da hier keine Zuordnung der Unfälle zu den Knotenarmen möglich war. Daher wurde auch kein Unfallmodell für die lichtsignalgeregelten Knotenpunkte erstellt.

Zur Überprüfung möglicher Einflüsse wurden daher in der Mikroanalyse die Radverkehrsstärken auf den zuführenden Streckenabschnitten in rechter Fahrtrichtung und die Unfälle von aus dieser Zufahrt kommenden Radfahrern betrachtet. Hierbei wurden zum einen alle Unfälle und zum anderen die Unfälle von Radfahrern in rechter Fahrtrichtung auf den jeweils vorgesehenen Radverkehrsanlagen betrachtet. Mit dieser zweiten Betrachtung sollten mögliche Einflüsse aus Fehlverhaltensweisen von Radfahrern - wie etwa der regelwidrigen Nutzung linker Furten - ausgeschlossen werden.

Schon bei niedrigen Radverkehrsstärken konnten bei Führungen auf Radwegen, Radfahrstreifen, im Mischverkehr sowie bei gemeinsamen Führungen des Rad- und Fußverkehrs hohe Unfallbelastungen auftreten. Ein Zusammenhang zwischen höheren Radverkehrsstärken und höheren Unfallbelastungen konnte weder für alle Unfälle noch für die Unfälle in rechter Fahrtrichtung auf der vorgesehenen Radverkehrsführung ermittelt werden (Bild 19, Bild 20). ${ }^{25}$ 


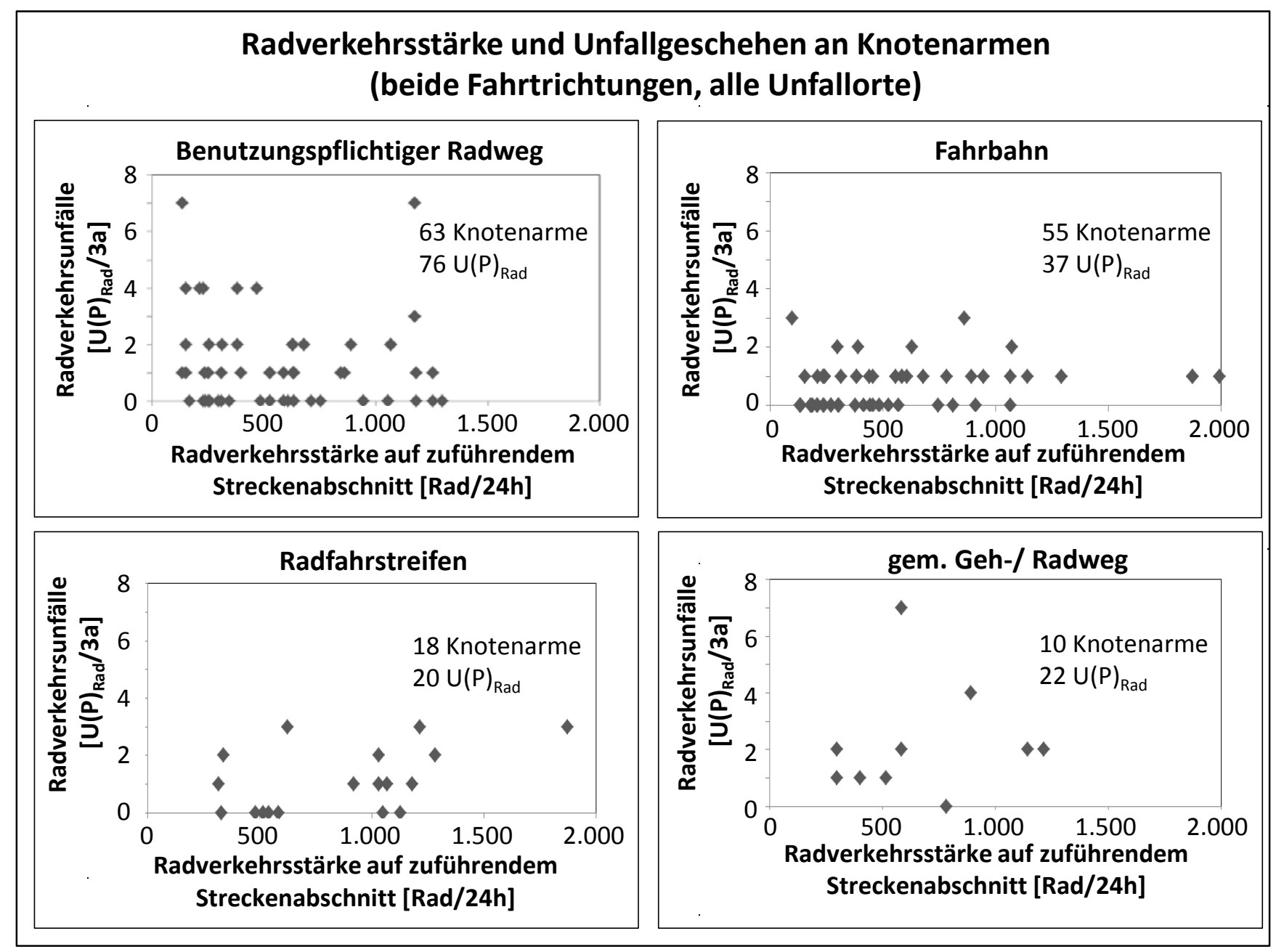

Bild 19: Radverkehrsstärken auf den zuführenden Streckenabschnitten lichtsignalgeregelter Knotenpunkte und Anzahl von Radfahrer-Unfällen mit Personenschaden in drei Jahren (beide Fahrtrichtungen, alle Unfallorte 


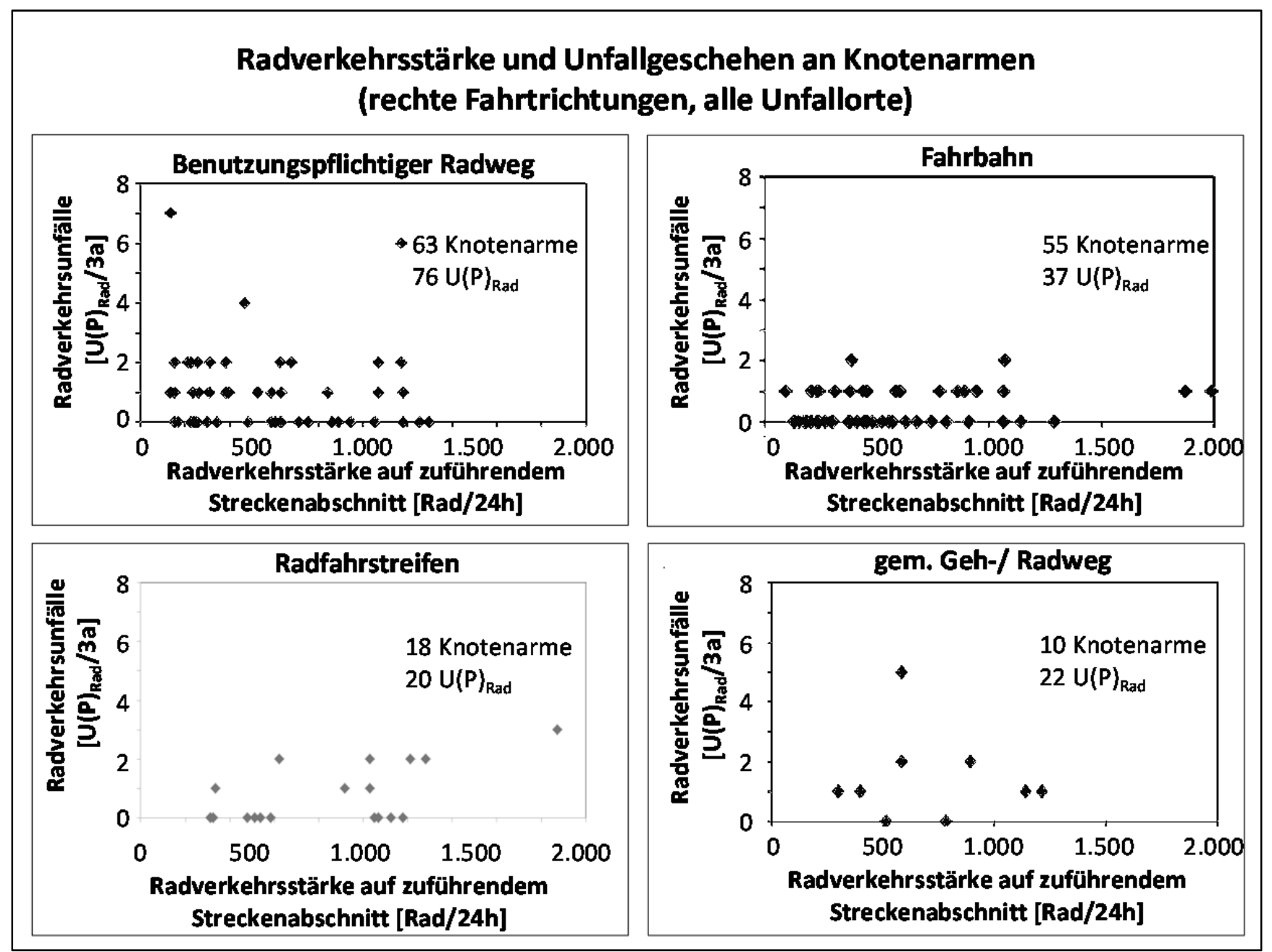

Bild 20: Radverkehrsstärken auf den zuführenden Streckenabschnitten lichtsignalgeregelter Knotenpunkte und Anzahl von Radfahrer-Unfällen mit Personenschaden in drei Jahren (rechte Fahrtrichtung, Unfallort auf vorgesehener Radverkehrsführung) 


\subsubsection{Verkehrsanteile und Unfallbeteiligung des Radverkehrs in ausgewählten Städten (Exkurs)}

Für zwanzig Städte, für die aus dem SrV 2008 Daten zur Verkehrsmittelwahl und den Verkehrsleistungen vorlagen, wurden ergänzend Zusammenhänge zwischen den Verkehrsanteilen des Radverkehrs und den Anteilen verletzter Radfahrer an allen verletzten Verkehrsteilnehmern untersucht. Sowohl nach den anteilig zurückgelegten Wegen (Verkehrsaufkommen) als auch nach den anteiligen Personenkilometern (Verkehrsleistung) des Radverkehrs stieg der Anteil der verletzten Radfahrer mit steigendem Anteil des Radverkehrs. Darüber hinaus lag der Anteil der verletzten Radfahrer nahezu immer über dem Anteil der Personenkilometer bzw. der Wegeanteile der Radfahrer (Bild 21 und Tabelle 26). Radfahrer haben demzufolge im Stadtverkehr ein höheres Verletzungsrisiko als andere Verkehrsteilnehmer. Bei zunehmendem Radverkehr nimmt folglich auch der Bedarf an Maßnahmen zur Sicherung von Radfahrern zu.

Mit zunehmendem Anteil an den Wegen nähert sich die Trendlinie der Quadrantenhalbierenden an. Das bestätigt den degressiven Anstieg der Unfallanzahl mit der Radverkehrsstärke, wie es sich im Unfallmodell ergab. Die Anzahl der Radverkehrsunfälle steigt demnach in geringerem Maße an als der Radverkehr. 


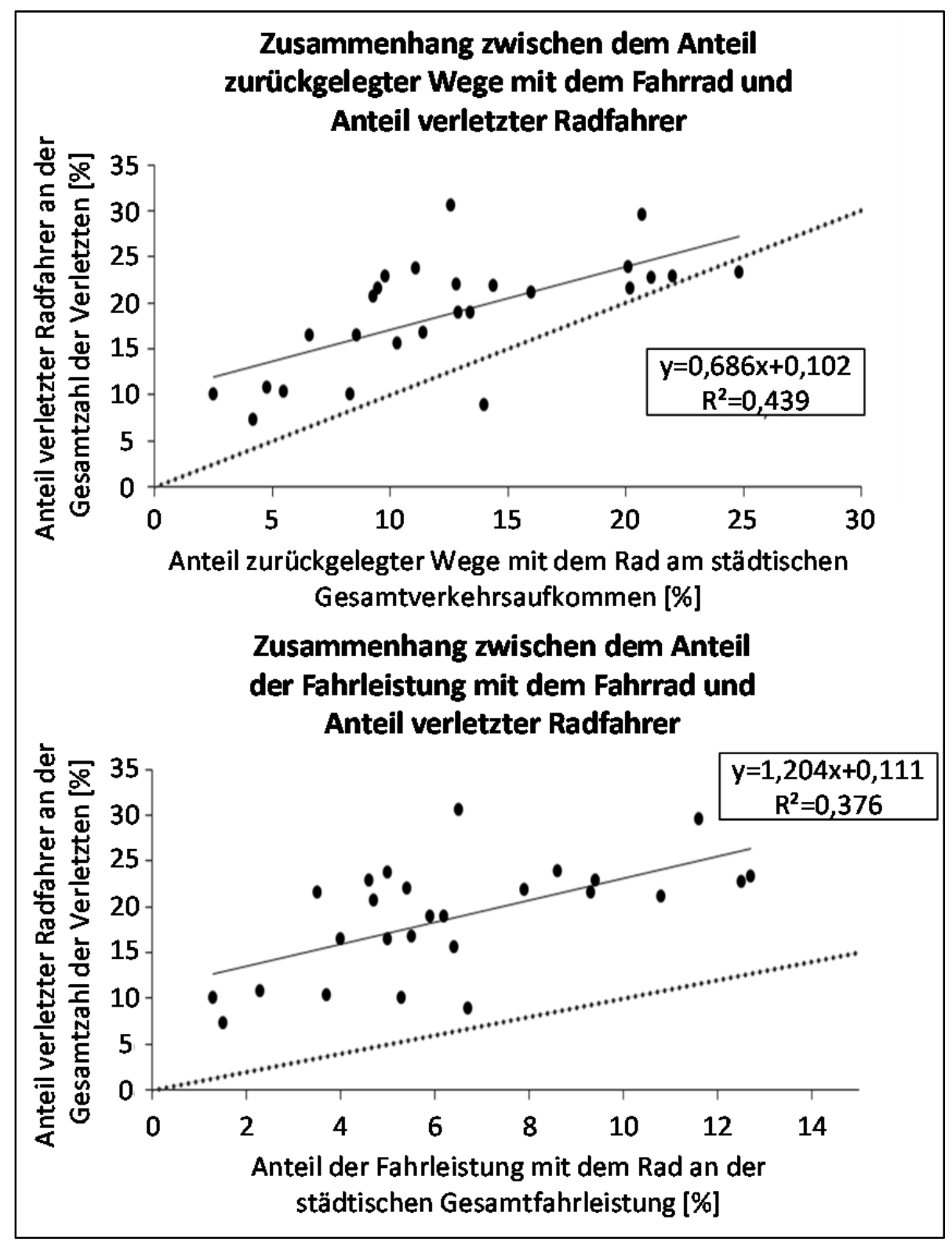

Bild 21: Zusammenhänge zwischen Verkehrsaufkommen, Verkehrsleistung und Verletztenzahlen ${ }^{26}$ 
Tabelle 26: Verkehrsanteile des Radverkehrs und Anteil an den Verletzten in den einzelnen Städten ${ }^{26}$

\begin{tabular}{|c|c|c|c|}
\hline Stadt & Anteil Wege [\%] & Anteil Personenkilometer [\%] & Anteil Verletzte [\%] \\
\hline Berlin & 12,6 & 6,5 & 30,6 \\
\hline \multicolumn{4}{|c|}{ bis 500.000 Einwohner } \\
\hline Frankfurt am Main & 12,9 & 6,2 & 18,9 \\
\hline Düsseldorf & 11,1 & 5,0 & 23,7 \\
\hline Bremen & 24,8 & 12,7 & 23,2 \\
\hline Leipzig & 14,4 & 7,9 & 21,9 \\
\hline Dresden & 16,0 & 10,8 & 21,2 \\
\hline \multicolumn{4}{|c|}{ bis 200.000 Einwohner } \\
\hline Mannheim & 12,8 & 5,4 & 22,0 \\
\hline Augsburg & 13,4 & 5,9 & 18,9 \\
\hline Chemnitz & 5,5 & 3,7 & 10,4 \\
\hline Kiel & 20,7 & 11,6 & 29,6 \\
\hline Halle (Saale) & 14,0 & 6,7 & 8,8 \\
\hline Magdeburg & 9,8 & 4,6 & 22,9 \\
\hline Rostock & 20,2 & 9,3 & 21,6 \\
\hline Erfurt & 8,3 & 5,3 & 10,1 \\
\hline Mainz & 9,5 & 3,5 & 21,6 \\
\hline \multicolumn{4}{|c|}{ bis 75.000 Einwohner } \\
\hline Kassel & 6,6 & 4,0 & 16,5 \\
\hline Ulm/Neu-Ulm & 11,4 & 5,5 & 16,8 \\
\hline Potsdam & 20,1 & 8,6 & 23,9 \\
\hline Fürth & 8,6 & 5,0 & 16,5 \\
\hline Jena & 10,3 & 6,4 & 15,6 \\
\hline Gera & 4,2 & 1,5 & 7,3 \\
\hline Kaiserslautern & 2,5 & 1,3 & 10,0 \\
\hline Cottbus & 21,1 & 12,5 & 22,7 \\
\hline Zwickau & 4,8 & 2,3 & 10,7 \\
\hline Schwerin & 9,3 & 4,7 & 20,7 \\
\hline Dessau-Roßlau & 22,0 & 9,4 & 22,9 \\
\hline
\end{tabular}




\subsubsection{Radverkehrsstärke und Unfallschwere}

\section{Streckenabschnitte}

Zur Beurteilung des Verkehrsstärkeeinflusses auf die Unfallschwere wurden in der Makroanalyse die Untersuchungsstrecken in zwei Untersuchungsklassen aufgeteilt. Dabei wurde zwischen den Strecken mit geringeren als im Mittel über alle Strecken der Untersuchung auftretenden Radverkehrsstärken (ca. $1.000 \mathrm{Rad} / 24 \mathrm{~h}$ ) und solchen mit überdurchschnittlichen Verkehrsstärken als dieser Mittelwert unterschieden. Entsprechend dieser Aufteilung der Erhebungsstrecken wurde das Unfallgeschehen mit Personenschaden und Radbeteiligung nach der Unfallkategorie (schwerste Unfallfolge) ausgewertet. Bild 22 zeigt die Unterschiede bei den Anteilen der Unfallkategorien in den zwei Klassen.

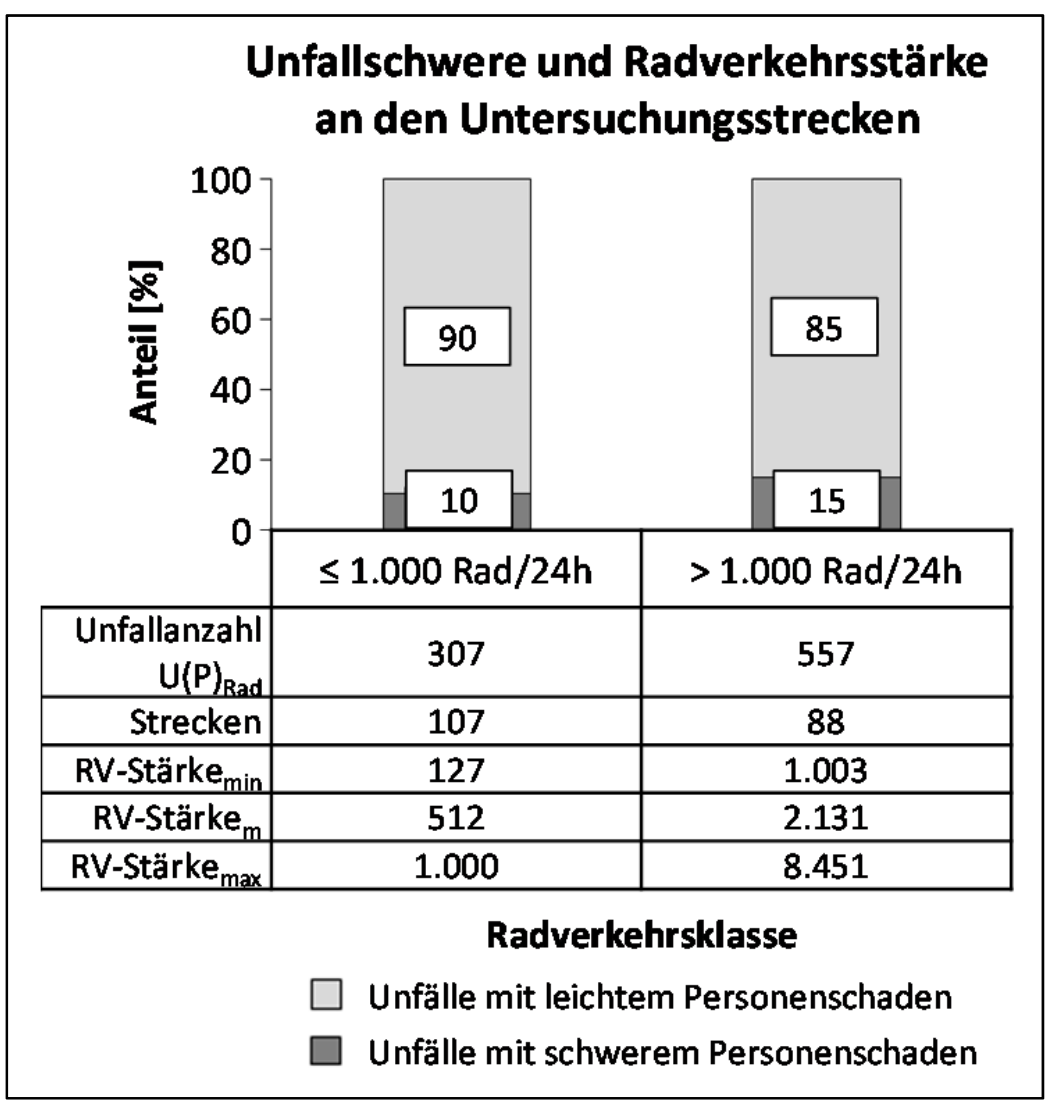

Bild 22: Unfallschwere nach Radverkehrsstärke in zwei Klassen

Tendenziell zeigte sich an den Erhebungsstrecken mit höheren Radverkehrsstärken auch ein höherer Anteil an schweren Unfällen. Der Anteil schwerer Radverkehrsunfälle von 10,3\% auf den Strecken mit geringen Radverkehrsstärken stieg auf den Strecken mit hohen Radverkehrsstärken auf $15 \%$ an. Hohe Anteile schwerer Unfälle an allen Unfällen mit Personenschaden konnten in Einzelfällen auf Strecken aber auch bereits bei vergleichsweise niedrigen Radverkehrsstärken auftreten. 
Für eine detailliertere Untersuchung der Unfallschwere auf den Strecken wurden die Unfallkostendichten den Radverkehrsstärken gegenübergestellt. Durch die Unfallkosten, werden sowohl die Unfallanzahl als auch die Unfallschwere beschrieben. Die Untersuchungen haben dabei nur für die straßenbündigen Führungsformen des Radverkehrs (Mischverkehr, Radfahrstreifen, Schutzstreifen und Bussonderfahrstreifen) einen Zusammenhang der Radverkehrsstärke mit der Unfallkostendichte gezeigt (Bild 23). Für die anderen Führungsformen zeigte die Radverkehrsstärke keinen signifikanten Zusammenhang zur Unfallkostendichte.

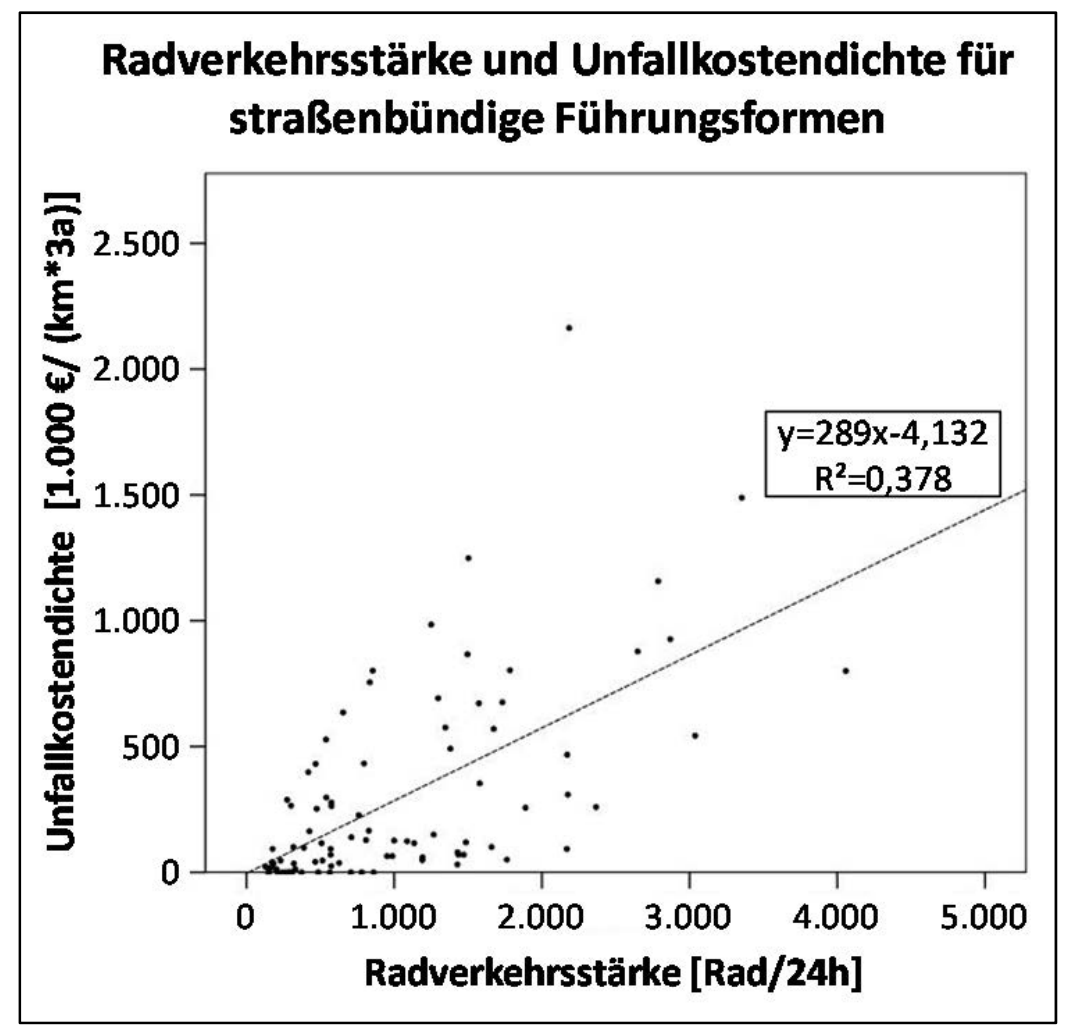

Bild 23: Radverkehrsstärken und Unfallkostendichte auf straßenbündigen Radverkehrsführungen (Mischverkehr, Radfahrstreifen, Schutzstreifen, Bussonderfahrstreifen)

Die Gegenüberstellung der Unfallkosten je Unfall zeigte jedoch keinen signifikanten Zusammenhang mit der Radverkehrsstärke (Bild 24). Abweichende hohe Unfallkosten je Unfall ergaben sich daher auf Strecken mit geringen Radverkehrsstärken aufgrund vereinzelter schwerer Unfälle bei einem geringen Unfallkollektiv. 


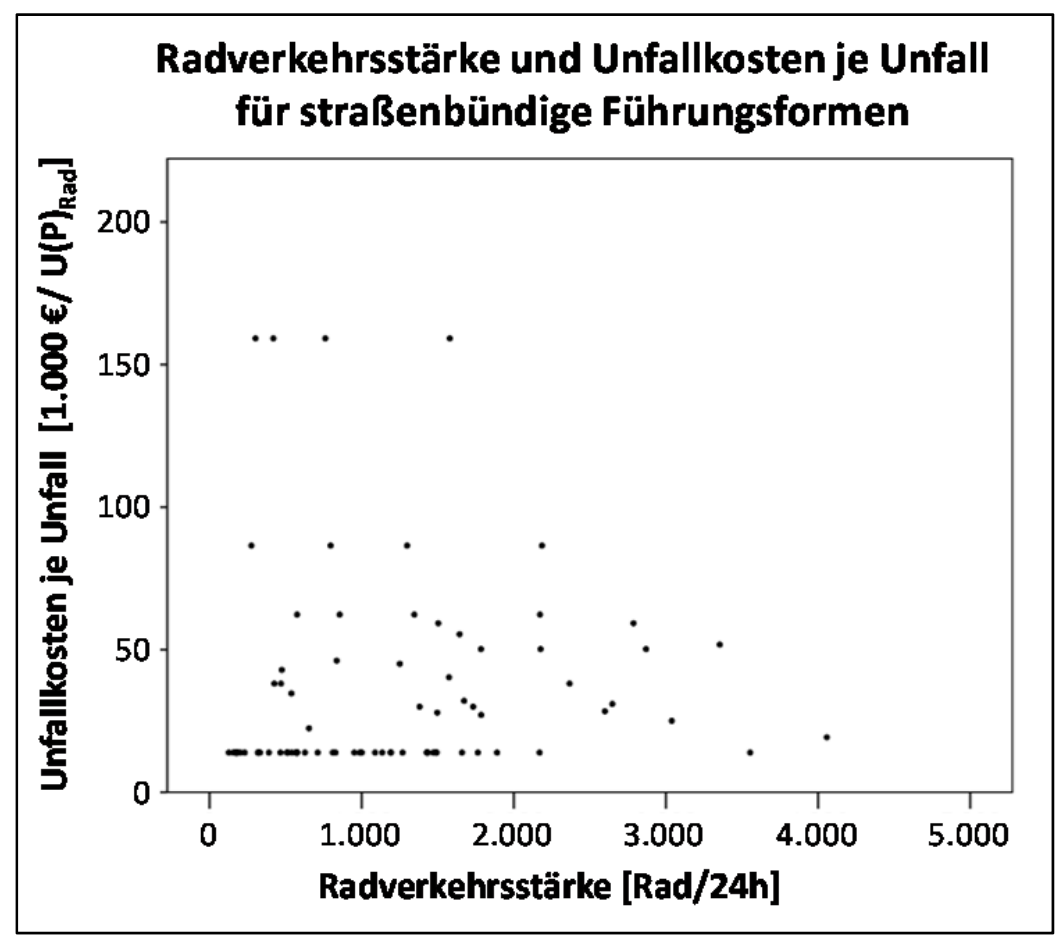

Bild 24: Radverkehrsstärken und Unfallkosten je Unfall auf straßenbündigen Radverkehrsführungen (Mischverkehr, Radfahrstreifen, Schutzstreifen, Bussonderfahrstreifen) 


\section{Lichtsignalgeregelte Knotenpunkte}

Wie auch bei der Betrachtung der Unfallanzahl, wurden in der Mikroanalyse zum einen alle Radverkehrsunfälle, zum anderen Radverkehrsunfälle in rechter Fahrtrichtung sowie Nutzung und Unfallort auf der vorgesehenen Radverkehrsführung herangezogen. Schon bei vergleichsweise niedrigen Radverkehrsstärken konnten dabei an den einzelnen Knotenarmen der verschiedenen Radverkehrsführungen hohe Anteile schwerer Unfälle an den Unfällen mit Personenschaden auftreten (Bild 25, Bild 26).

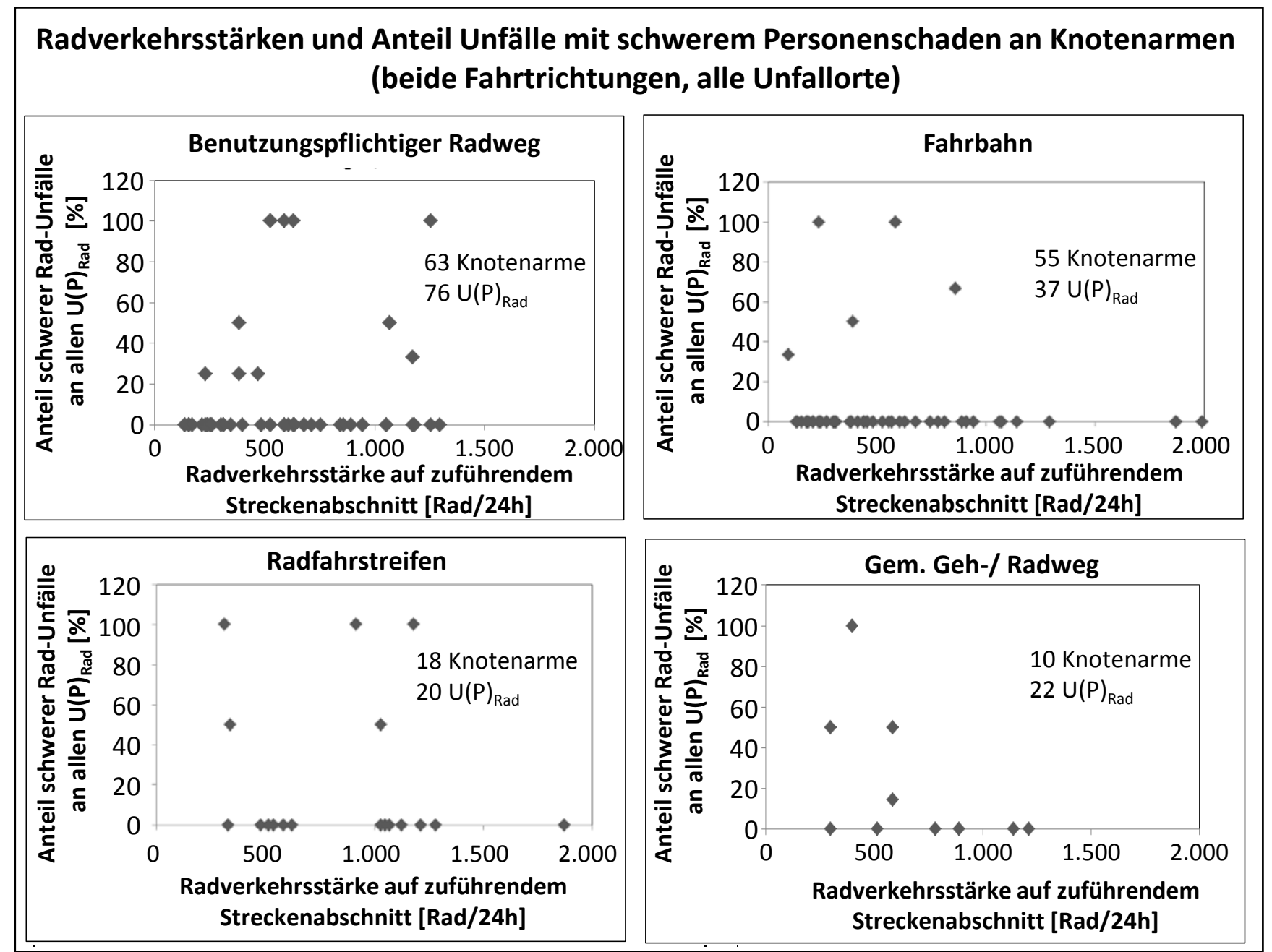

Bild 25: Radverkehrsstärke und Anteil von Unfällen mit schwerem Personenschaden an allen Unfällen mit Personenschaden an lichtsignalgeregelten Knotenpunkten (beide Fahrtrichtungen, alle Unfallorte, einschließlich unfallfreie Knotenzufahrten) 
Radverkehrsstärken und Anteil Unfälle mit schwerem Personenschaden an Knotenarmen (rechte Richtung, Nutzung der vorgesehenen Verkehrsflächen)
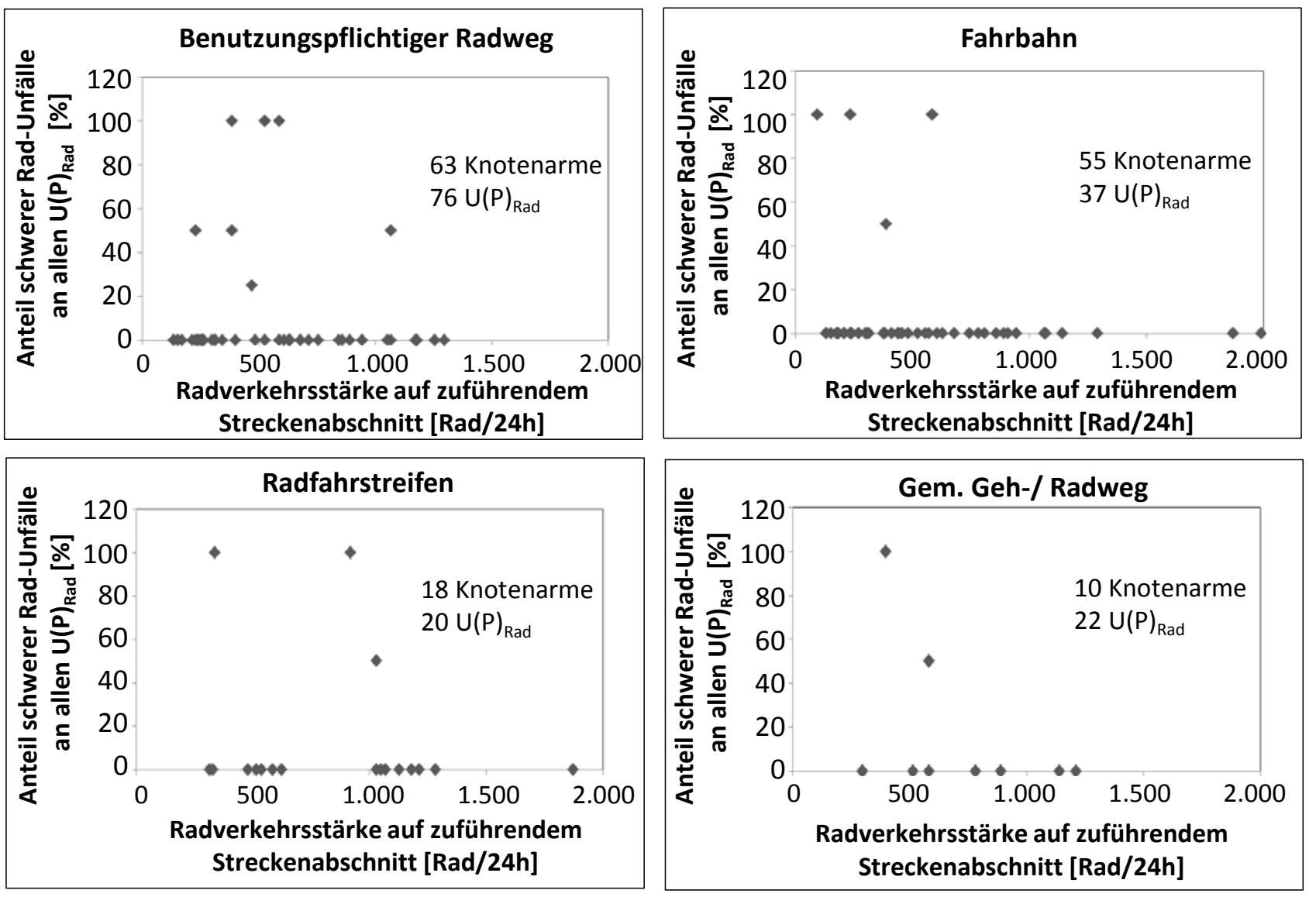

Bild 26: Radverkehrsstärke und Anteil von Unfällen mit schwerem Personenschaden an allen Unfällen mit Personenschaden an lichtsignalgeregelten Knotenpunkten (rechte Fahrtrichtung, Unfallort: vorgesehen Radverkehrsführung, einschließlich unfallfreie Knotenzufahrten)

Die in Bild 25 und Bild 26 dargestellten Verteilungen wurden daher regressionsanalytisch überprüft. Ein verallgemeinerbarer Zusammenhang zwischen Radverkehrsstärken und Unfallschwere konnte dabei an lichtsignalgeregelten Knotenpunkten nicht ermittelt werden (maximales Bestimmtheitsmaß $\left.\mathrm{R}^{2}=0,1\right)$.

Der Mittelwert der Radverkehrsstärken auf den zuführenden Streckenabschnitten in Fahrtrichtung des Knotenpunktes lag bei $600 \mathrm{Rad} / 24 \mathrm{~h}$. Bei einer Unterteilung in die Klassen von bis zu und über $600 \mathrm{Rad} / 24 \mathrm{~h}$ traten bei höheren Radverkehrsstärken um acht Prozentpunkte niedrigere Anteile von Unfällen mit schwerem Personenschaden auf (Bild 27). Bei ausschließlicher Betrachtung der Unfälle in rechter Fahrtrichtung und auf den vorgesehenen Radverkehrsführungen lag der Anteil von Unfällen mit schwerem Personenschaden bei höheren Radverkehrsstärken sogar um 12 Prozentpunkte niedriger (Bild 28). 


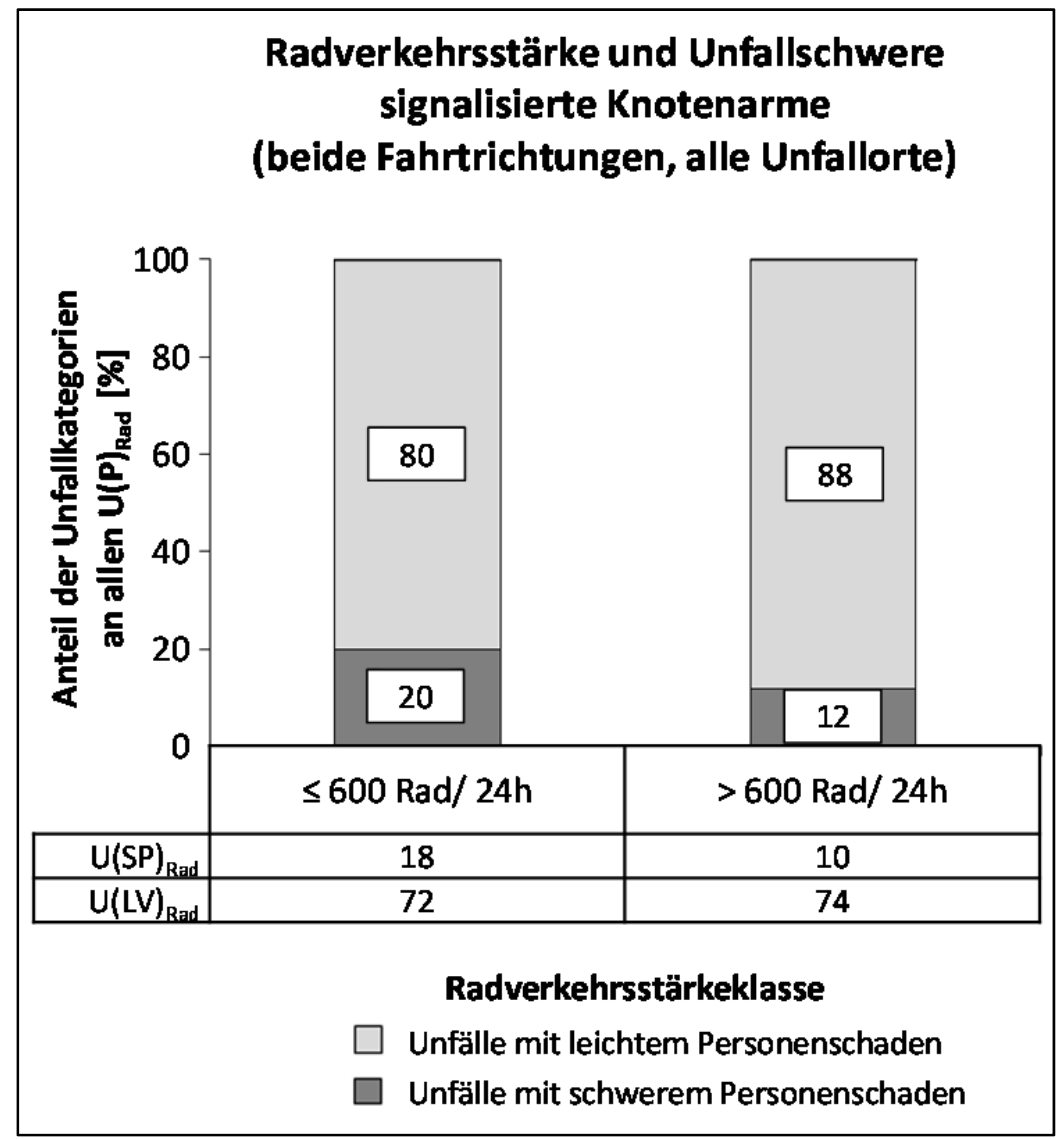

Bild 27: Radverkehrsstärken auf den zuführenden Streckenabschnitten und Unfallschwere an lichtsignalgeregelten Knotenpunkten (beide Fahrtrichtungen, alle Unfallorte) 


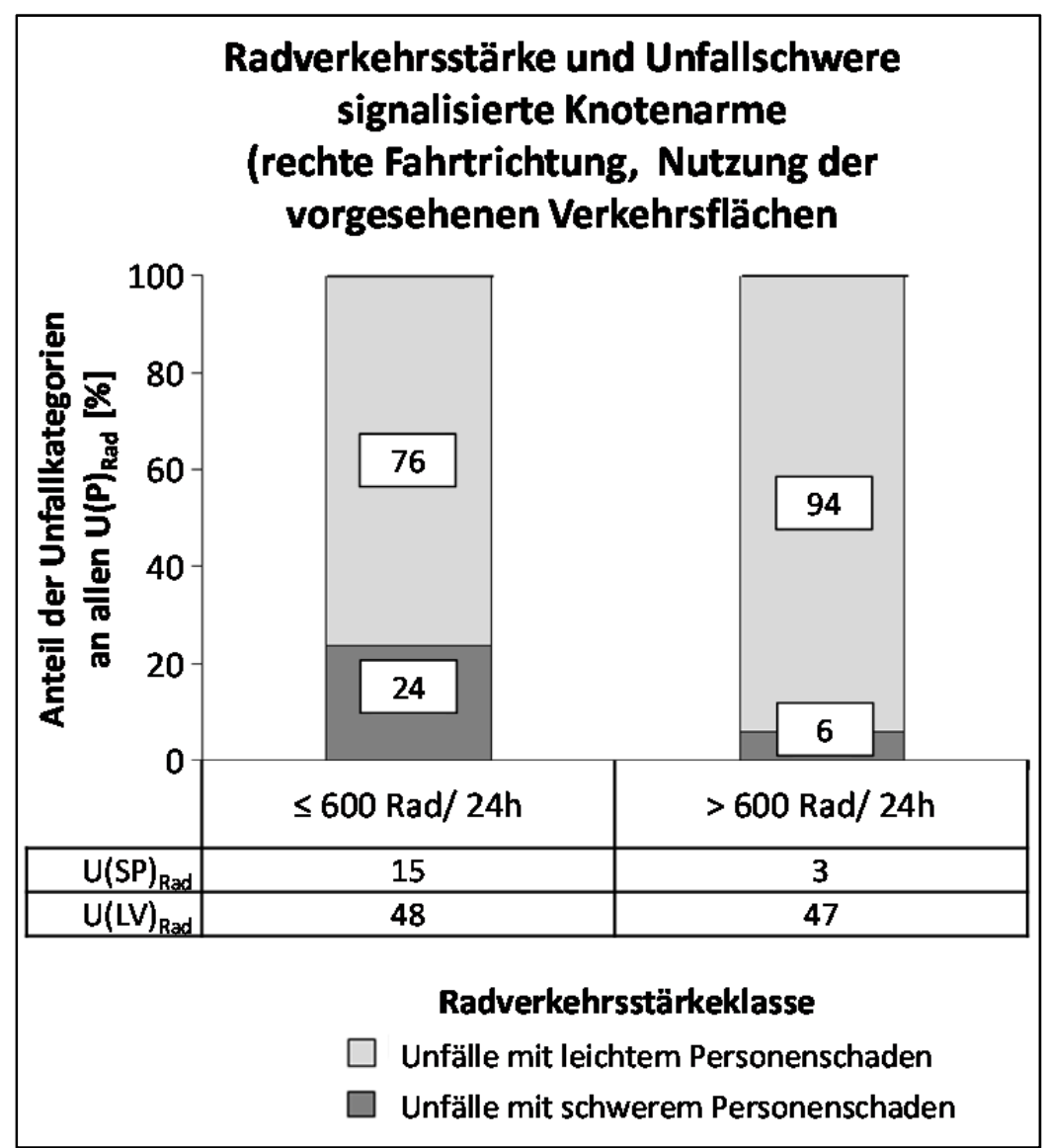

Bild 28: Radverkehrsstärken auf den zuführenden Streckenabschnitten und Unfallschwere an lichtsignalgeregelten Knotenpunkten (rechte Fahrtrichtung und Unfallorte auf der vorgesehenen Radverkehrsfläche)

\section{Unfallraten an lichtsignalgeregelten Knoten}

An den lichtsignalgeregelten Knotenarmen lagen die mittleren Unfallraten bei gemeinsamen Führungen des Rad- und Fußverkehrs und bei Radwegführungen deutlich höher als bei Fahrbahn- oder Radfahrstreifenführungen (Bild 29). Die mittleren Unfallkostenraten hingegen lagen bei Fahrbahn, Führung auf dem Radfahrstreifen und auf Radwegen in etwa auf einem Niveau (Bild 30). Dies erklärt sich dadurch, dass sich bei gemeinsamer Führung des Rad- und Fußverkehrs anteilig häufiger schwerere Unfälle ereigneten. Hierdurch erklären sich auch die steigenden Unfallkostenraten des auf der Fahrbahn geführten Radverkehrs und Radfahrstreifens gegenüber der Radwegführung. 


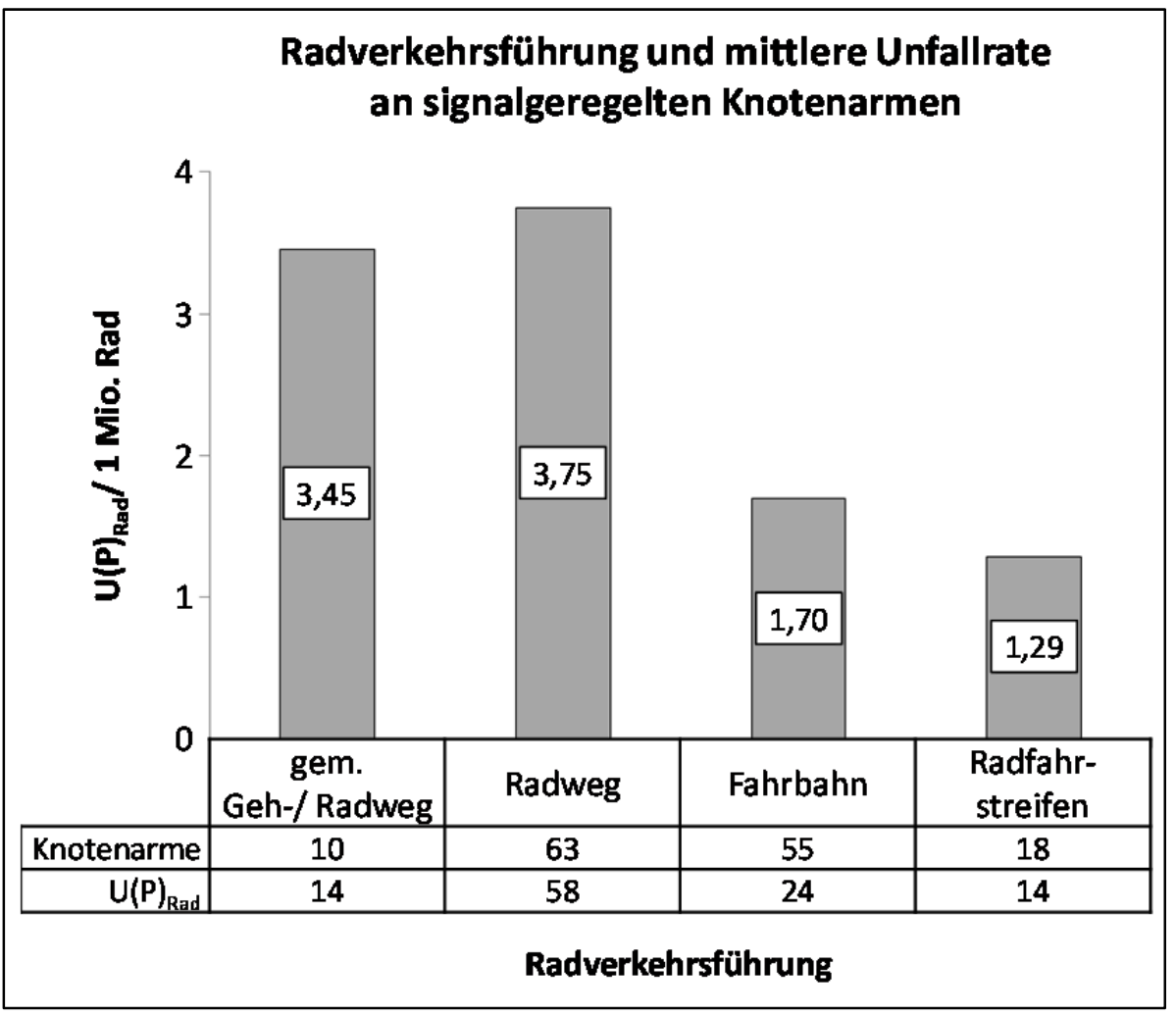

Bild 29: Mittlere Unfallrate nach Führungsform an lichtsignalgeregelten Knotenpunkten

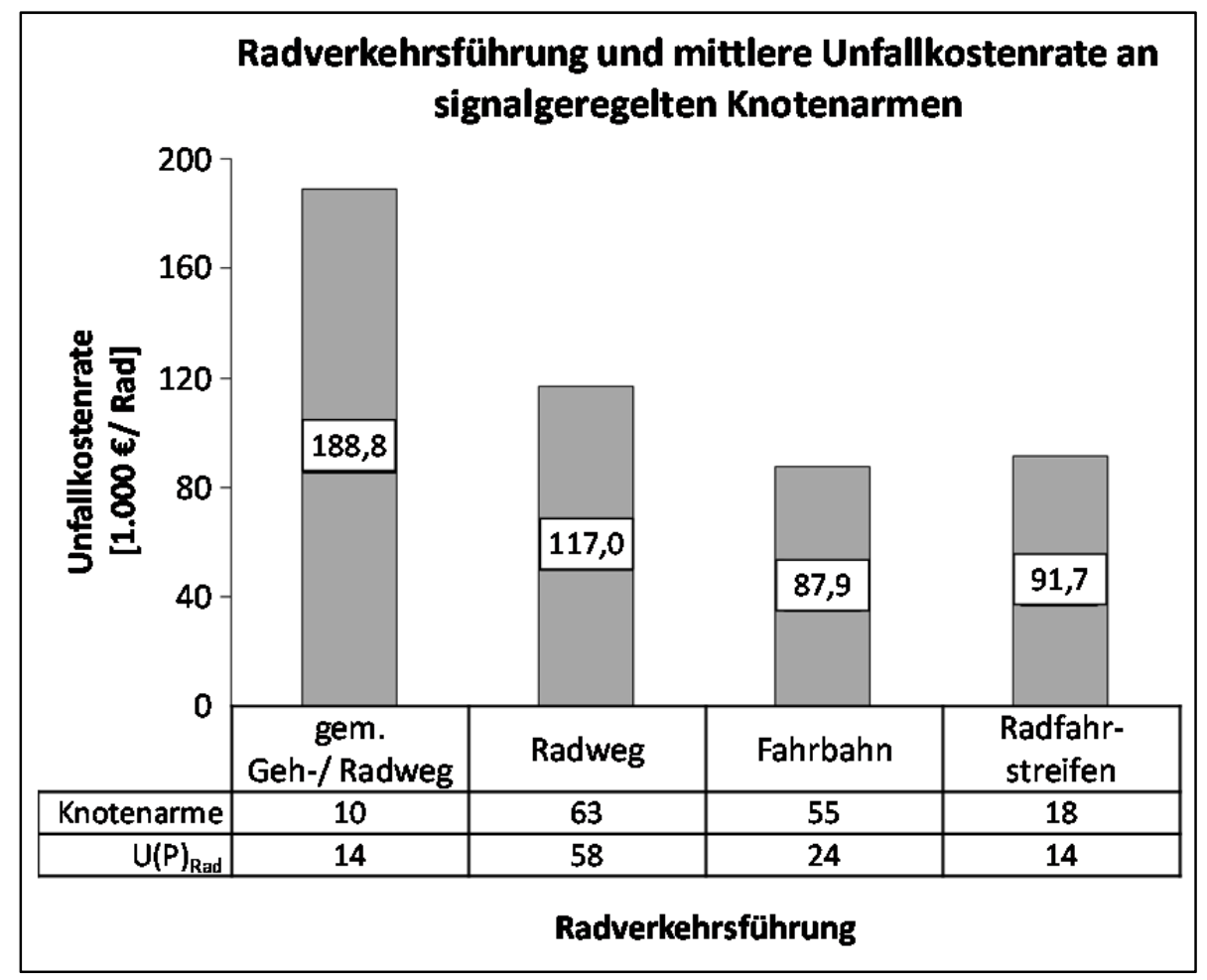

Bild 30:

Mittlere Unfallkostenrate nach Führungsform an lichtsignalgeregelten Knotenpunkten 


\subsubsection{Radverkehrsstärke und Unfalltypenverteilung}

\section{Streckenabschnitte}

Mit der zuvor beschriebenen Aufteilung der Erhebungsstrecken nach ihrer Radverkehrsstärke (anhand des Mittelwerts in zwei Klassen) wurden die Verteilungen der Unfalltypen in der Makroanalyse untersucht. Um Verschiebungen in den Anteilen der relevanten Unfalltypen zu verdeutlichen, wurden Unfälle des Typs 7 „Sonstige“ nicht mit betrachtet ${ }^{27}$. Die Unfalltypen „Einbiegen-/ Kreuzen“ und „Abbiegen" traten auch bei Streckenabschnitten aufgrund der hier verwendeten Definition von Streckenabschnitten als Verbindung zwischen Verkehrsknotenpunkten und somit auch Anschlussknotenpunkte auf.

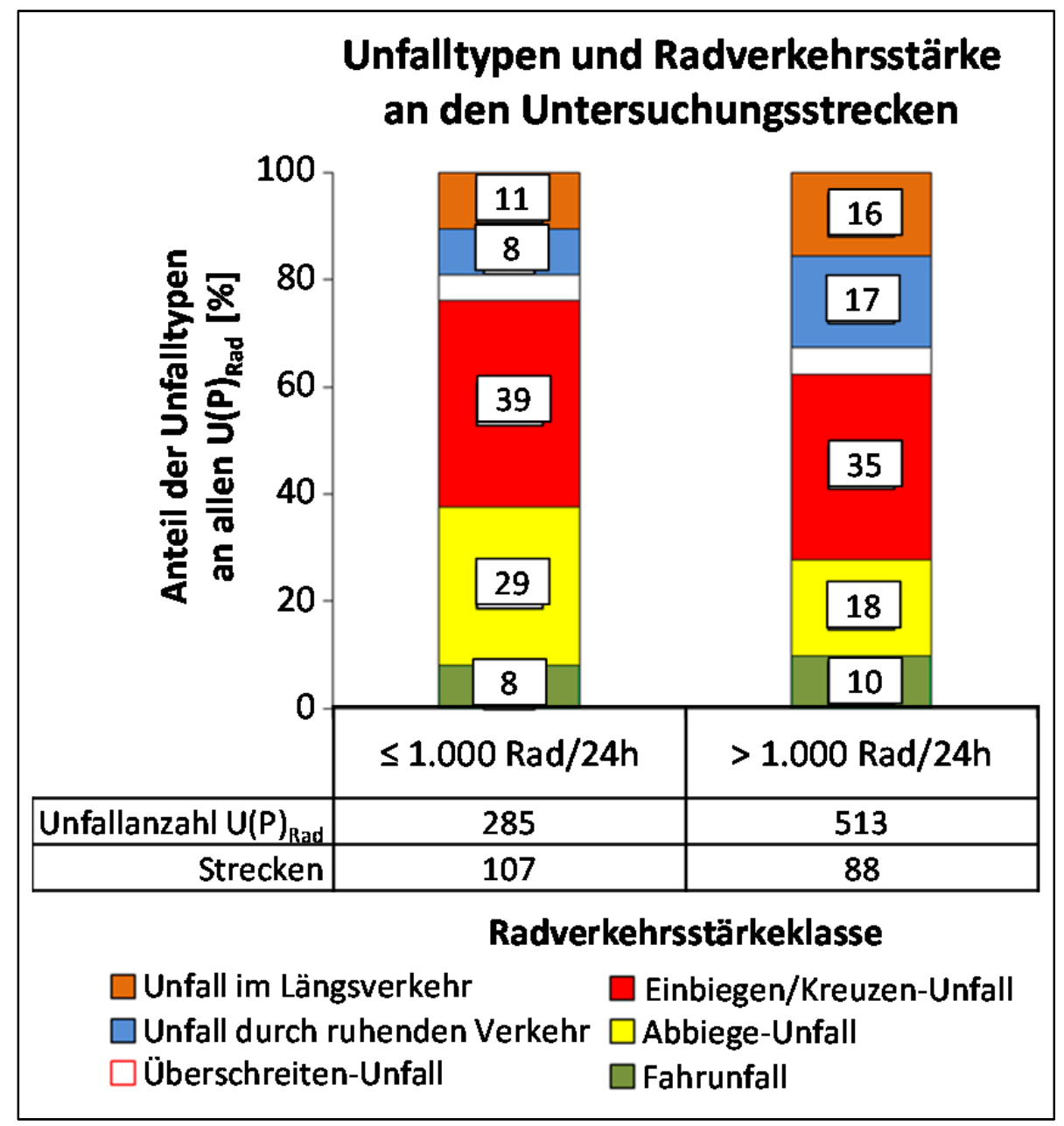

Bild 31: Unfalltypen (Anteile, ohne Typ 7) und Radverkehrsstärke in zwei Klassen

Mit höheren Radverkehrsstärken verschoben sich die Unfalltypen anteilig zu Unfällen im Längsverkehr (11 \% auf $16 \%)$ und zu Unfällen durch ruhenden Verkehr (8\% auf $17 \%$ ). Der Anteil der knotenpunktspezifischen Typen „Abbiegen“ und „Einbiegen-/ Kreuzen“ ging demzufolge etwas zurück (in der Summe von $68 \%$ auf $53 \%$ ) (Bild 31). 
Die Verschiebungen der Unfalltypen ließen sich jedoch nicht alle auf den Einfluss der Radverkehrsstärke zurückführen. Zwischen beiden Radverkehrsstärkeklassen existierten systematische Unterschiede. Diese resultierten aus

- der Verwendung anderer Führungsformen bei hohen Radverkehrsstärken (siehe Anhang 7.3),

- dem unterschiedlichen Anstieg der Unfälle mit der Radverkehrsstärke je nach Führungsform (siehe Kapitel 4.2.1) und

- der unterschiedlichen Unfalltypenverteilung je nach Führungsform (siehe Anhang 7.3)

Anhang 7.3 zeigt hierzu die Verteilung der Führungsformen, der Unfälle und der Unfalltypen auf die Radverkehrsstärkeklassen.

Bezüglich ihrer Führungsformen ergaben sich in der Radverkehrsstärkeklasse „> 1.000 Rad/24h“ weniger Schutzstreifen und weniger Führungen des Radverkehrs auf der Fahrbahn im Kollektiv. Da auf den Führungsformen der Anstieg der Unfälle mit der Radverkehrsstärke je nach Führungsform in unterschiedlichem Maß ausfällt, führte der Anstieg der Radverkehrsstärke anteilig zu mehr Unfällen auf dem Radfahrstreifen und anteilig weniger auf dem Schutzstreifen und dem Radweg.

Die Gegenüberstellung der Unfalltypen auf den Führungsformen zeigte für alle Führungsformen einen anteiligen Anstieg der Längsverkehrsunfälle in der Radverkehrsstärkeklasse „> $1.000 \mathrm{Rad} / 24 \mathrm{~h}$ “. Demnach konnte ein anteiliger Anstieg der Längsverkehrsunfälle auf den Anstieg der Radverkehrsstärken zurückgeführt werden. Die Veränderungen der Anteilswerte der anderen Unfalltypen im Gesamtkollektiv der Strecken („Abbiegen“, „Einbiegen-/ Kreuzen“ und „Ruhender Verkehr“) ergaben sich dagegen aus der veränderten Verteilung der Führungsformen in den beiden Radverkehrsstärkeklassen.

Als Ergebnis der Mikroanalyse schlüsselt die Tabelle 27 die dreistelligen Unfalltypen nach M Uko (FGSV, 2012) nach Radverkehrsanlage und Radverkehrsstärkenklasse auf. Die Radverkehrsstärken wurden dabei nach dem Mittelwert der Abschnitte der Mikroanalyse eingeteilt. Gerade für die in der Makroanalyse ermittelte Verschiebung zu Unfällen im Längsverkehr zeigte sich:

- Auf Strecken mit Radwegführung und höheren Radverkehrsstärken ereigneten sich anteilig mehr Unfälle zwischen begegnenden Radfahrern im Längsverkehr (Typ 681). Die Unfälle dieses Typs ereigneten sich dabei auf Radwegen mit weniger als 1,6 $\mathrm{m}$ Breite.

- Bei Fahrbahnführung und höheren Radverkehrsstärken geschahen anteilig mehr Unfälle zwischen Radfahrern und überholenden Kfz (Typ 651). Auffällig war hier ein Streckenabschnitt mit zwei Richtungsfahrstreifen. 
Bei anderen Führungsformen konnten unabhängig von den Radverkehrsstärken auffällig häufig Unfallabläufe festgestellt werden, die durch bauliche Merkmale einzelner unfallauffälliger Untersuchungsabschnitte beeinflusst waren. Hierzu zählten z. B.

- sonstige Fahrunfälle bei Fahrbahnführung (Typ 199 - Alleinunfälle durch Stürze in Straßenbahngleisen),

- Unfälle zwischen rechts einbiegenden $\mathrm{Kfz}$ und bevorrechtigtem Radfahrer auf dem Radfahrstreifen (Typ 303) an Einmündungen mit weiten Kurvenradien,

- Unfälle zwischen kreuzenden Radfahrern und Kfz in Längsrichtung (Typ 371) auf einer Straße mit Radfahrstreifen,

- Unfälle zwischen regelwidrig linksfahrendem Radfahrer auf Radwegen und einbiegendem Kfz (Typ 342), die sich ausgeprägt auch an Parkplatzausfahrten von Einzelhandelsmärkten ereignen

- oder Unfälle durch ruhenden Verkehr bei Radfahr- sowie bei Schutzstreifen beim Ein-/Aussteigen (Typ 581).

Die in den beiden Radverkehrsstärkeklassen unterschiedlich hohen Anteile der Unfalltypen 302 und 342 bei Mischverkehrsführung standen vor dem Hintergrund eines niedrigen Unfallkollektivs und deuten daher nicht auf ein speziellen Einfluss höherer Radverkehrsstärken. 
Tabelle 27: Anteile der Unfalltypen nach Radverkehrsanlage und Radverkehrsstärkenklasse an Streckenabschnitten

\begin{tabular}{|c|c|c|c|c|c|c|c|c|c|c|c|c|c|c|}
\hline \multirow{3}{*}{ 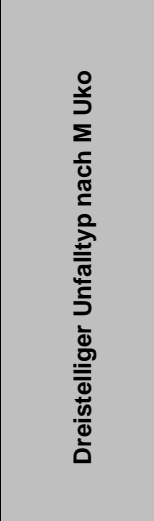 } & \multicolumn{14}{|c|}{ Radverkehrsanlage } \\
\hline & \multicolumn{2}{|c|}{ 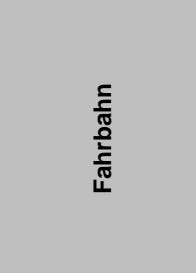 } & \multicolumn{2}{|c|}{ 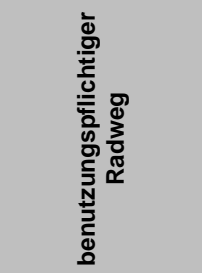 } & \multicolumn{2}{|c|}{ 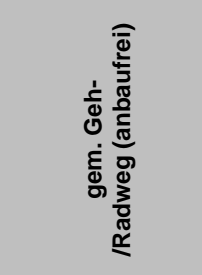 } & \multicolumn{2}{|c|}{ 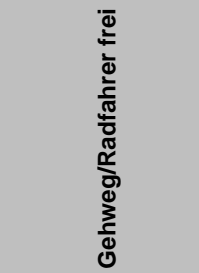 } & \multicolumn{2}{|c|}{ 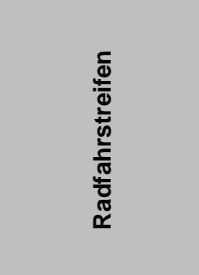 } & \multicolumn{2}{|c|}{ 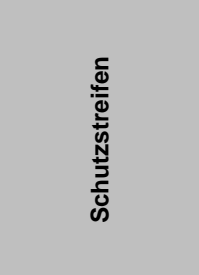 } & \multicolumn{2}{|c|}{ 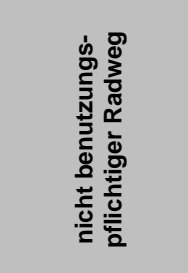 } \\
\hline & 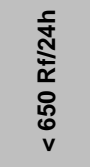 & 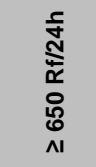 & 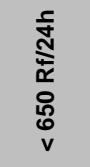 & 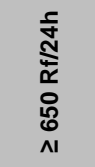 & 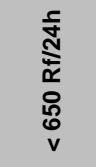 & 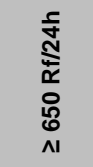 & 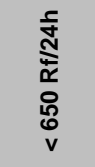 & 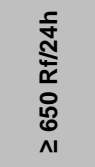 & 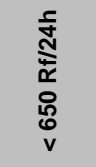 & 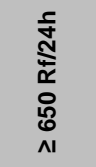 & 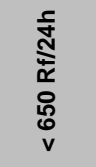 & 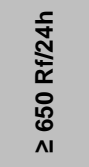 & 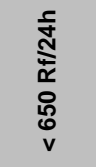 & 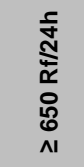 \\
\hline 199 & $5 \%$ & $32 \%$ & $5 \%$ & $2 \%$ & $67 \%$ & $67 \%$ & & $17 \%$ & $10 \%$ & $12 \%$ & & & & \\
\hline 203 & & & & $1 \%$ & & & & & & & & & $6 \%$ & \\
\hline 204 & & $3 \%$ & & & & & & & & & $22 \%$ & & & \\
\hline 211 & $5 \%$ & & & & & & & & & $5 \%$ & $22 \%$ & $5 \%$ & & \\
\hline 223 & & & & $2 \%$ & & & & & & & & & & \\
\hline 224 & & & $4 \%$ & $2 \%$ & & & & & & & & & $6 \%$ & \\
\hline 232 & $7 \%$ & $6 \%$ & & & & & & & $10 \%$ & $15 \%$ & & $5 \%$ & & \\
\hline 243 & & & $14 \%$ & $17 \%$ & & & & & & & & & $18 \%$ & \\
\hline 244 & & & $2 \%$ & & & & & & & & & & $6 \%$ & \\
\hline 299 & $5 \%$ & & $2 \%$ & $1 \%$ & & & & & $3 \%$ & & & & & \\
\hline 301 & $12 \%$ & & & & & & & & & $2 \%$ & $11 \%$ & & & \\
\hline 302 & $7 \%$ & $12 \%$ & & & & & & & & $2 \%$ & & & & \\
\hline 303 & & $3 \%$ & & & & & & & $24 \%$ & $10 \%$ & & $5 \%$ & & \\
\hline 311 & & & & & & & & & & & & & $12 \%$ & \\
\hline 321 & & & $1 \%$ & $3 \%$ & & & & & & & & & $12 \%$ & $100 \%$ \\
\hline 323 & & & & $1 \%$ & & & & & & & & & & \\
\hline 341 & & & $12 \%$ & $7 \%$ & & & $100 \%$ & & & & & & & \\
\hline 342 & $10 \%$ & $3 \%$ & $27 \%$ & $17 \%$ & & & & & & $29 \%$ & & $19 \%$ & $29 \%$ & \\
\hline 371 & $2 \%$ & $3 \%$ & $4 \%$ & $1 \%$ & & & & $33 \%$ & $14 \%$ & $2 \%$ & $22 \%$ & $10 \%$ & & \\
\hline 372 & $2 \%$ & & $6 \%$ & $7 \%$ & & & & & & & & $5 \%$ & & \\
\hline 399 & $5 \%$ & & & $1 \%$ & & & & & & & & $5 \%$ & & \\
\hline 401 & $2 \%$ & & $1 \%$ & $3 \%$ & & & & & & & & $5 \%$ & & \\
\hline 421 & $2 \%$ & & $3 \%$ & $3 \%$ & & $33 \%$ & & & $3 \%$ & & & $10 \%$ & & \\
\hline 499 & & & $1 \%$ & & & & & & & & & $5 \%$ & & \\
\hline 501 & $2 \%$ & $3 \%$ & & & & & & $17 \%$ & & & & & & \\
\hline 541 & $2 \%$ & & & & & & & & & & & & & \\
\hline 542 & & & & & & & & & & $2 \%$ & & & & \\
\hline 551 & $2 \%$ & & & & & & & & & $2 \%$ & & & & \\
\hline 581 & $15 \%$ & $15 \%$ & $4 \%$ & & & & & & $17 \%$ & $5 \%$ & $22 \%$ & $19 \%$ & & \\
\hline 582 & & & $1 \%$ & & & & & & & & & $5 \%$ & & \\
\hline 584 & $2 \%$ & & & & & & & & & & & & & \\
\hline 601 & & & & & & & & & $3 \%$ & $2 \%$ & & & & \\
\hline 631 & & $3 \%$ & & $1 \%$ & & & & & $3 \%$ & $2 \%$ & & & & \\
\hline 651 & $2 \%$ & $9 \%$ & $4 \%$ & $3 \%$ & & & & $17 \%$ & & $5 \%$ & & $5 \%$ & $6 \%$ & \\
\hline 681 & & & $4 \%$ & $19 \%$ & & & & & & & & & & \\
\hline 699 & $2 \%$ & $3 \%$ & $2 \%$ & $1 \%$ & & & & $17 \%$ & & & & & & \\
\hline 721 & & $3 \%$ & & $1 \%$ & & & & & & & & & & \\
\hline 775 & $2 \%$ & & & & & & & & & & & & & \\
\hline 799 & $2 \%$ & $3 \%$ & $4 \%$ & $3 \%$ & $33 \%$ & & & & $10 \%$ & $2 \%$ & & & $6 \%$ & \\
\hline$\Sigma$ & $100 \%$ & $100 \%$ & $100 \%$ & $100 \%$ & $100 \%$ & $100 \%$ & $100 \%$ & $100 \%$ & $100 \%$ & $100 \%$ & $100 \%$ & $100 \%$ & $100 \%$ & $100 \%$ \\
\hline Anzahl U (P) & 41 & 34 & 113 & 88 & 3 & 3 & 1 & 6 & 29 & 41 & 9 & 21 & 17 & 1 \\
\hline
\end{tabular}




\section{Lichtsignalgeregelte Knotenpunkte}

In der Mikroanalyse der Unfälle an lichtsignalgeregelten Knotenpunkten wurden die dreistelligen Unfalltypen nach dem M UKo (FGSV, 2012) für die unterschiedlichen Führungsformen betrachtet ${ }^{28}$. Dabei zeigten sich keine belastbaren Einflüsse der Radverkehrsstärkeklasse auf die Unfalltypen. Dass einzelne Unfalltypen auffällig hohe Anteile von $25 \%$ (oder mehr) an den Unfällen eines Anlagentyps und einer Radverkehrsstärkenklasse aufwiesen, war zumeist durch niedrige Unfallzahlen beeinflusst (Tabelle 28).

Tabelle 28: Unfalltypen nach Führung in der Knotenzufahrt und Radverkehrsstärkeklasse (Anteile nach Führungsform und Verkehrsstärke)

\begin{tabular}{|c|c|c|c|c|c|c|c|c|}
\hline \multirow{3}{*}{ 胥 } & \multicolumn{8}{|c|}{ Führung Zufahrt } \\
\hline & \multicolumn{2}{|c|}{$\begin{array}{l}\frac{c}{\frac{c}{\pi}} \\
\frac{0}{2} \\
\frac{c}{\pi} \\
\frac{1}{4}\end{array}$} & \multicolumn{2}{|c|}{ 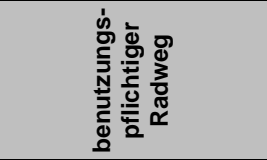 } & \multicolumn{2}{|c|}{ 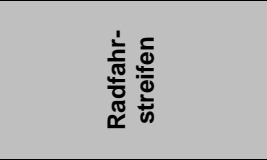 } & \multicolumn{2}{|c|}{ 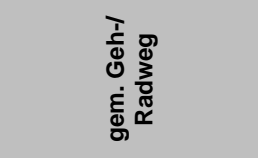 } \\
\hline & 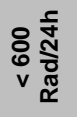 & 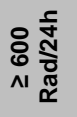 & 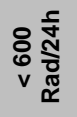 & 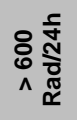 & ○ & 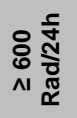 & 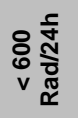 & 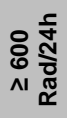 \\
\hline 199 & $5 \%$ & $13 \%$ & $7 \%$ & $4 \%$ & & $23 \%$ & & \\
\hline 203 & & & $2 \%$ & & & & & \\
\hline 204 & $5 \%$ & & & & & & & \\
\hline 211 & $10 \%$ & $6 \%$ & & & & $23 \%$ & & \\
\hline 223 & $5 \%$ & & & & $17 \%$ & & & \\
\hline 224 & & & $5 \%$ & & & & $14 \%$ & $13 \%$ \\
\hline 232 & $14 \%$ & $6 \%$ & & $4 \%$ & $33 \%$ & $15 \%$ & & \\
\hline 243 & & & $52 \%$ & $46 \%$ & & & $38 \%$ & $36 \%$ \\
\hline 244 & $5 \%$ & $19 \%$ & $9 \%$ & & $17 \%$ & & $14 \%$ & \\
\hline 299 & $5 \%$ & & & & & & & $13 \%$ \\
\hline 301 & $10 \%$ & $6 \%$ & & & & $8 \%$ & & \\
\hline 302 & $10 \%$ & $19 \%$ & & & $33 \%$ & & & \\
\hline \multicolumn{9}{|l|}{303} \\
\hline 341 & & & $5 \%$ & $19 \%$ & & & $36 \%$ & $25 \%$ \\
\hline 342 & $10 \%$ & & $5 \%$ & $4 \%$ & & & & \\
\hline 372 & $5 \%$ & & & & & & & \\
\hline 401 & & & $5 \%$ & & & & & \\
\hline 421 & $5 \%$ & & $2 \%$ & & & & & \\
\hline 581 & & & & & & $8 \%$ & & \\
\hline 582 & & & & $4 \%$ & & & & \\
\hline 599 & & & & & & $8 \%$ & & \\
\hline 601 & & & & & & $8 \%$ & & \\
\hline 631 & & & $2 \%$ & & & & & \\
\hline 651 & $14 \%$ & $25 \%$ & $2 \%$ & $4 \%$ & & & & \\
\hline 671 & & & $2 \%$ & & & & & \\
\hline 681 & $4 \%$ & & & $4 \%$ & & $8 \%$ & & \\
\hline 799 & & & $2 \%$ & $8 \%$ & & & & $7 \%$ \\
\hline Anzahl U (P) & 21 & 16 & 44 & 26 & 6 & 13 & 8 & 14 \\
\hline
\end{tabular}




\subsubsection{Zusammenhänge zwischen Radverkehrsstärke und Regelbefolgung}

\section{Streckenabschnitte}

Gemäß der durchgeführten Verhaltensbeobachtung lag der Anteil regelwidrig links fahrender Radfahrer für Straßen mit Radwegen durchschnittlich bei 12 bis $15 \%$. Die Anteile der regelwidrig links Fahrenden streuten dabei auf den einzelnen Straßenseiten in diesen Straßen sehr stark. Als höchster Anteil regelwidrig links Fahrender wurden auf zwei Straßen etwa 34 \% ermittelt. Bei Straßen mit Radfahrstreifen, Schutzstreifen oder für den Radverkehr frei gegebenen Bussonderfahrstreifen wurde ermittelt, dass durchschnittlich etwa $6 \%$ aller Radfahrer in regelwidriger linker Richtung fuhren (Bild 32).

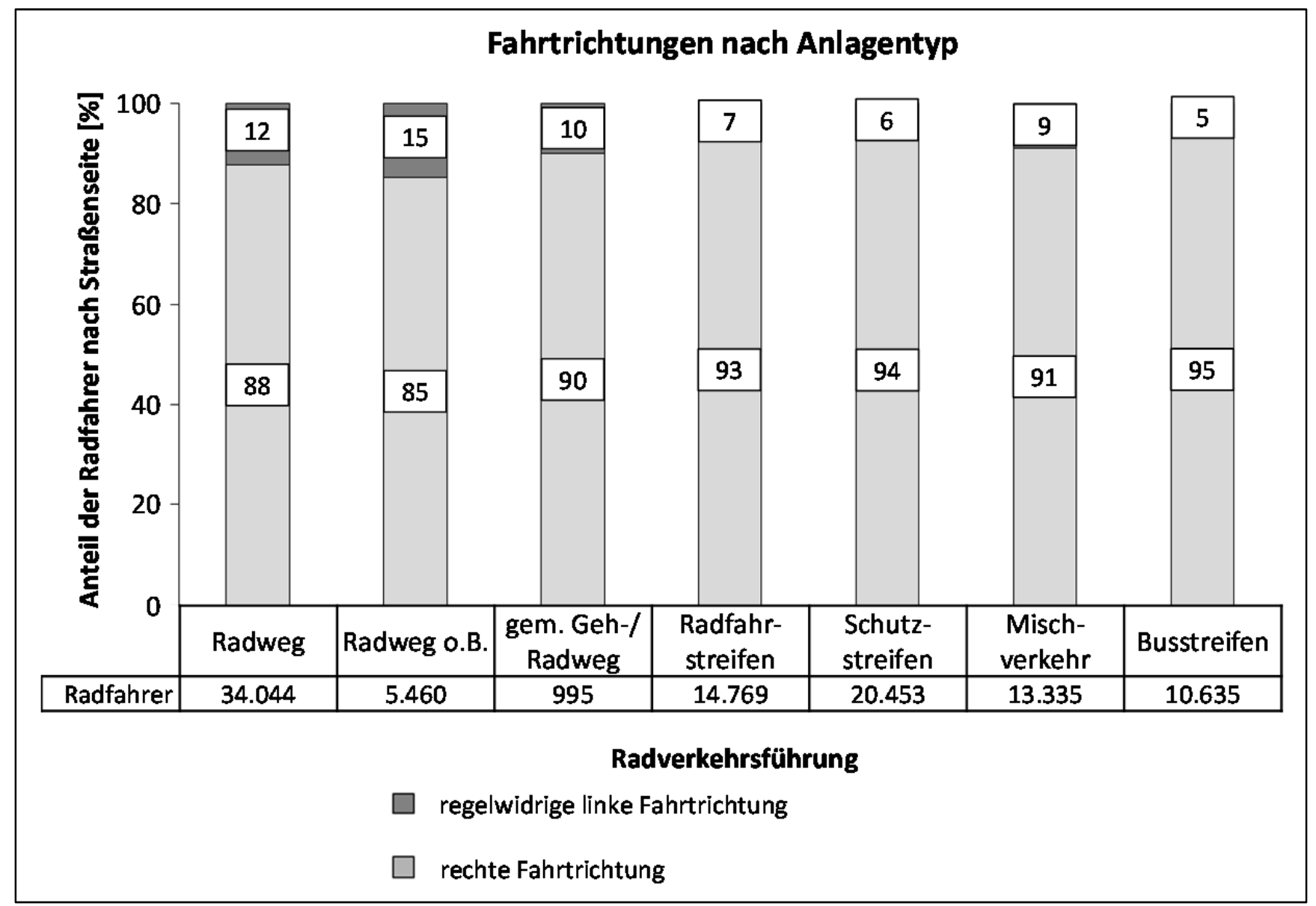

Bild 32: Anteile der rechten und regelwidrigen linken Fahrtrichtung nach Anlagentyp (ohne Differenzierung nach Flächennutzung) 
Wurden Anlagen mit hohen und niedrigen Radverkehrsstärken verglichen, so hatten Strecken mit höheren Radverkehrsstärken niedrigere Anteile regelwidrig linksfahrender Radfahrer vorzuweisen. Bei bis zu $1.000 \mathrm{Rad} / \mathrm{Tag}$ je Straßenseite fuhren durchschnittlich $16 \%$ der Radfahrer regelwidrig links, bei über $2.000 \mathrm{Rad} / \mathrm{Tag}$ nur noch $9 \%$ der Radfahrer (Bild 33).

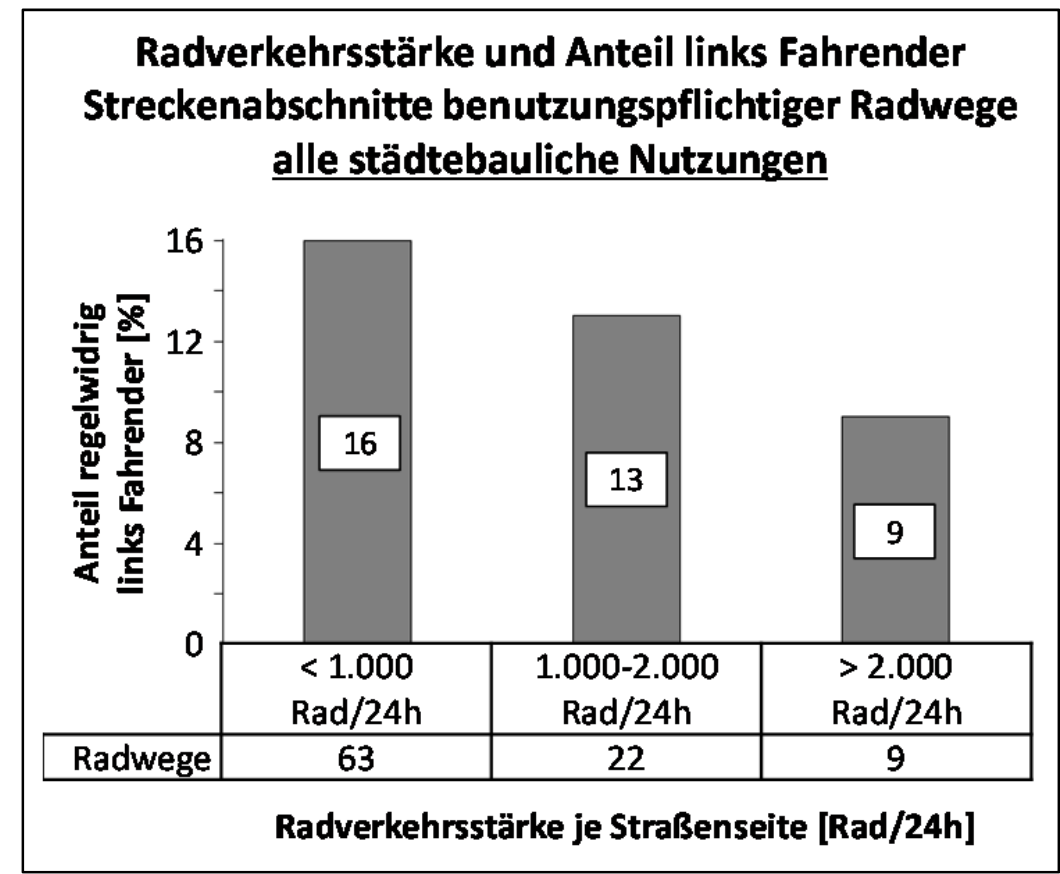

Bild 33: Radverkehrsstärken und Anteil regelwidrig links Fahrender in Straßen mit benutzungspflichtigen Radwegen, alle Städtebaulichen Nutzungen 
Hohe Anteile regelwidrig links Fahrender zeigten sich gehäuft in Straßen mit Einzelhandels- und Mischnutzungen. Dabei fuhren oftmals auf einer der beiden Straßenseiten mehr Radfahrer regelwidrig links als auf der gegenüberliegenden Straßenseite. Auch bei einer Differenzierung nach den städtebaulichen Nutzungen zeigte sich ein Einfluss der Radverkehrsstärke auf den Anteil regelwidrig links Fahrender: Bei Einzelhandels- und Mischnutzungen etwa sank der Anteil regelwidrig links Fahrender in den o. g. Verkehrsstärkenbereichen von $15 \%$ auf $10 \%$ (Bild 34).

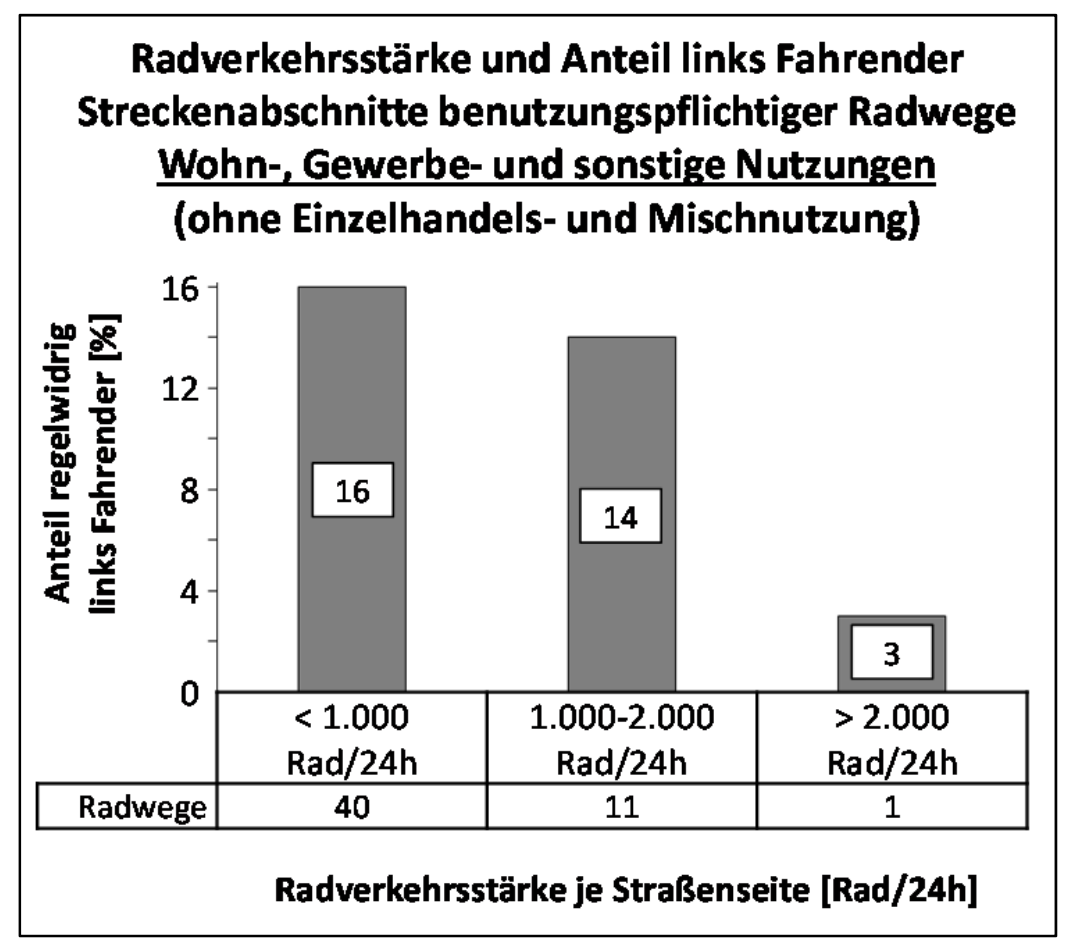

Bild 34: Radverkehrsstärken und Anteil regelwidrig links Fahrender in Straßen mit benutzungspflichtigen Radwegen, Einzelhandels- und Mischnutzungen 
Bei mehr als 4.000 Radfahrern je Tag und Straßenseite mit diesen städtebaulichen Nutzungen zeigten sich sehr niedrige Linksfahreranteile von unter $3 \%$. Auch bei anderen städtebaulichen Nutzungen zeigte sich ein Rückgang des regelwidrigen Linksfahrens mit der Radverkehrsstärke (Bild 35).

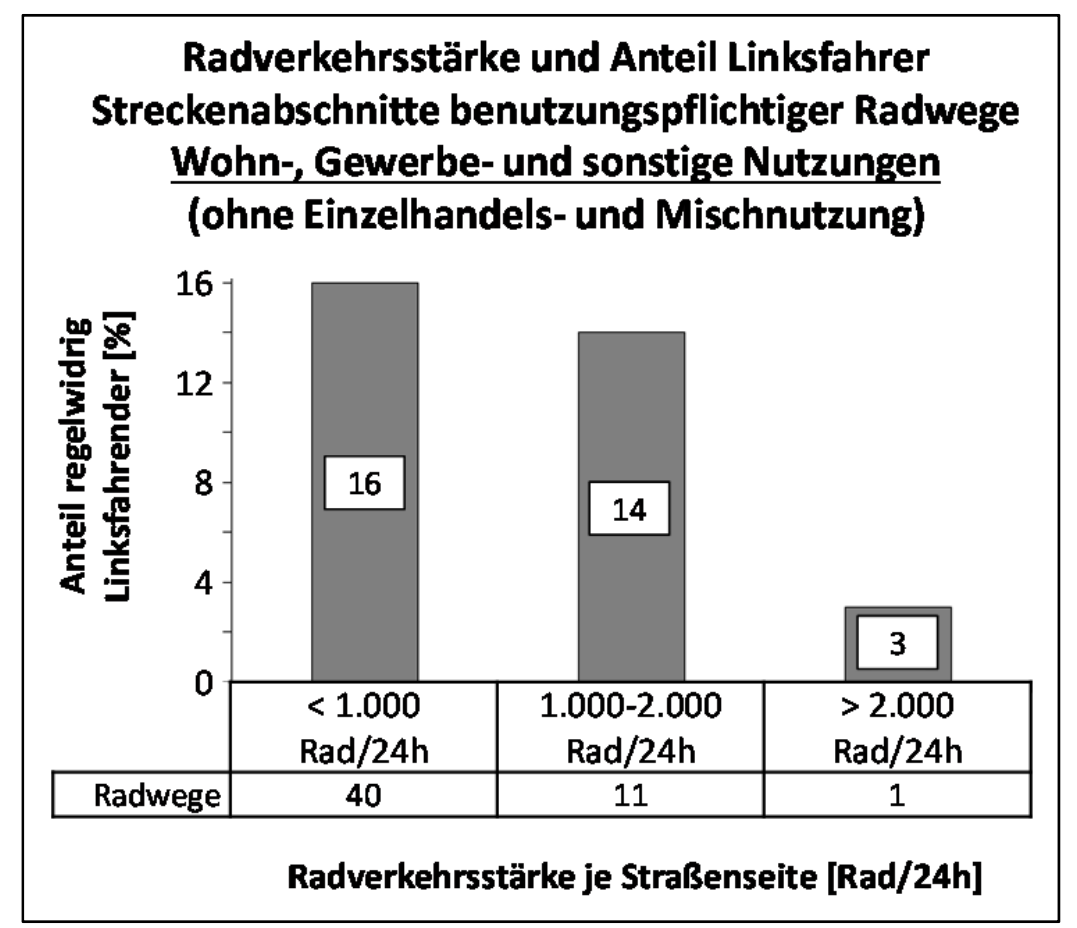

Bild 35: Radverkehrsstärken und Anteil regelwidrig links Fahrender in Straßen mit benutzungspflichtigen Radwegen. Wohn, Gewerbe- und Sonstige Nutzungen ohne Einzelhandels- und Mischnutzungen 
Sicherheitsprobleme durch das regelwidrige Linksfahren bestanden vor allem bei Radwegen. Die auch aus anderen Untersuchungen bekannte erhöhte Gefährdung regelwidrig links Fahrender bestätigte sich, denn die Unfallraten waren für die linke Fahrrichtung mehr als doppelt so hoch wie für die rechte Fahrtrichtung (Bild 36). Allerdings wirkte diese erhöhte Unfallgefährdung nicht auf eine erhöhte Unfallschwere hin: $9 \%$ der Unfälle mit Personenschaden regelwidrig links Fahrender hatten schwere Verletzungsfolgen, 11 \% der Unfälle mit Personenschaden von Radfahrern in rechter Richtung.

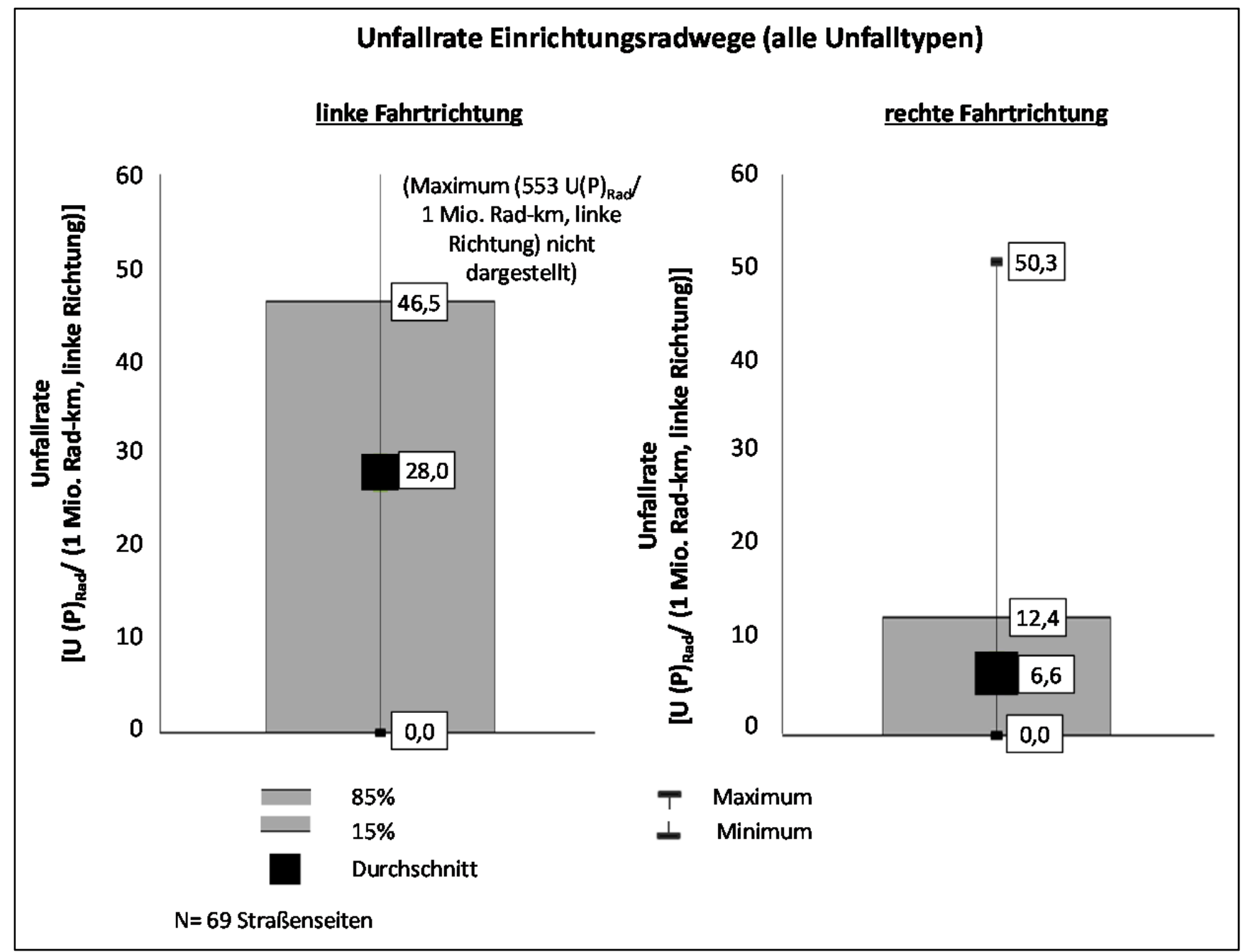

Bild 36: Unfallraten in linker und rechter Fahrtrichtung ${ }^{29}$ 
Bereits bei geringen Stärken des regelwidrigen linken Radverkehrs waren auffällig hohe Unfalldichten regelwidrig links Fahrender möglich. Zwischen der Anzahl regelwidrig links Fahrender und der Unfalldichte links Fahrender konnte jedoch kein Zusammenhang ermittelt werden (Bild 37). Die weitaus meisten Unfälle in linker Richtung ereigneten sich bei Radwegnutzung (Bild 38).

Auch für nicht benutzungspflichtige Radwege zeigte sich ein vergleichbares Bild. In Straßen mit Radfahr- oder Schutzstreifen fuhren die regelwidrig links Fahrenden weitaus überwiegend auf den Gehwegen. Hier traten insgesamt sehr selten Unfälle links Fahrender auf.

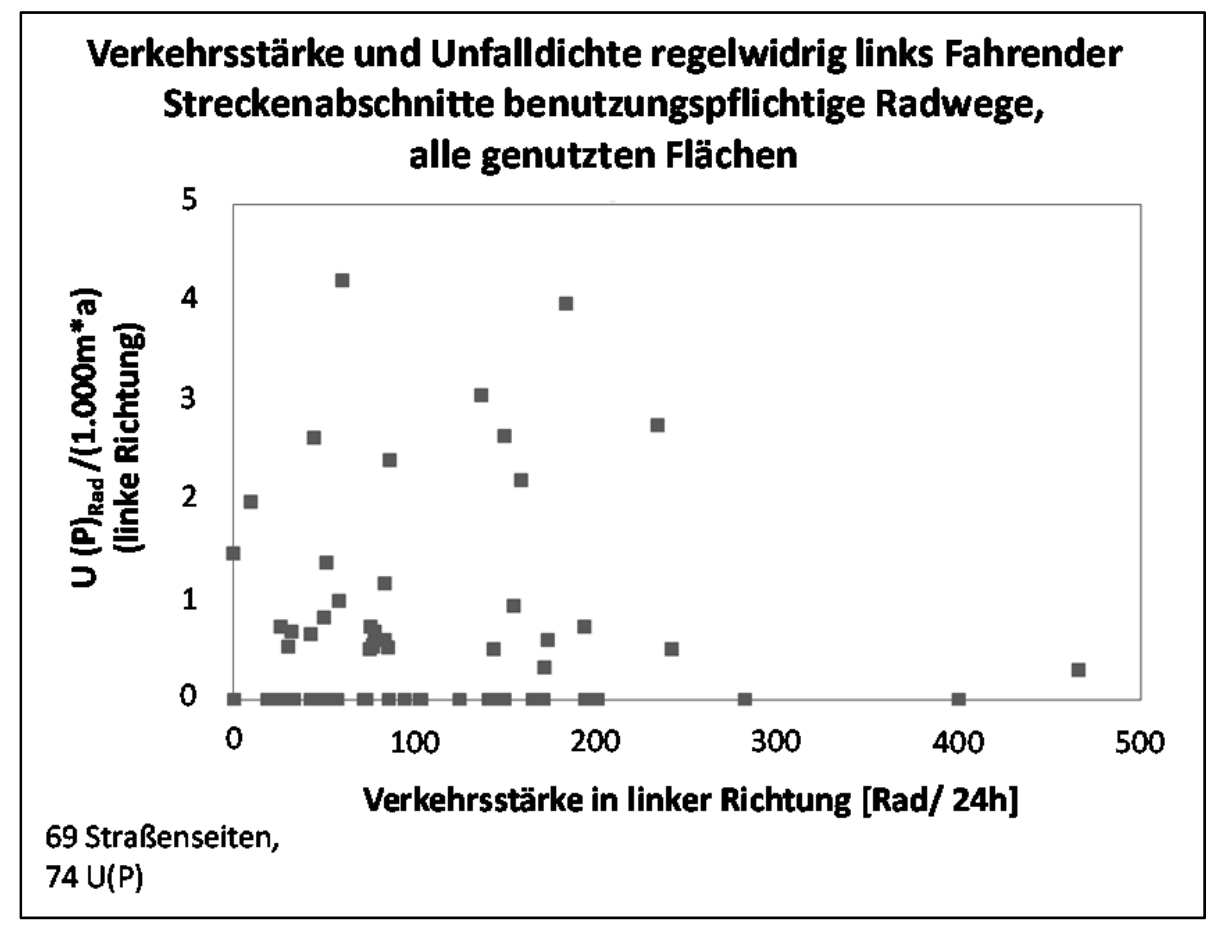

Bild 37: Anzahl regelwidrig links Fahrender und Unfalldichte links Fahrender in Straßen mit benutzungspflichtigen Radwegen

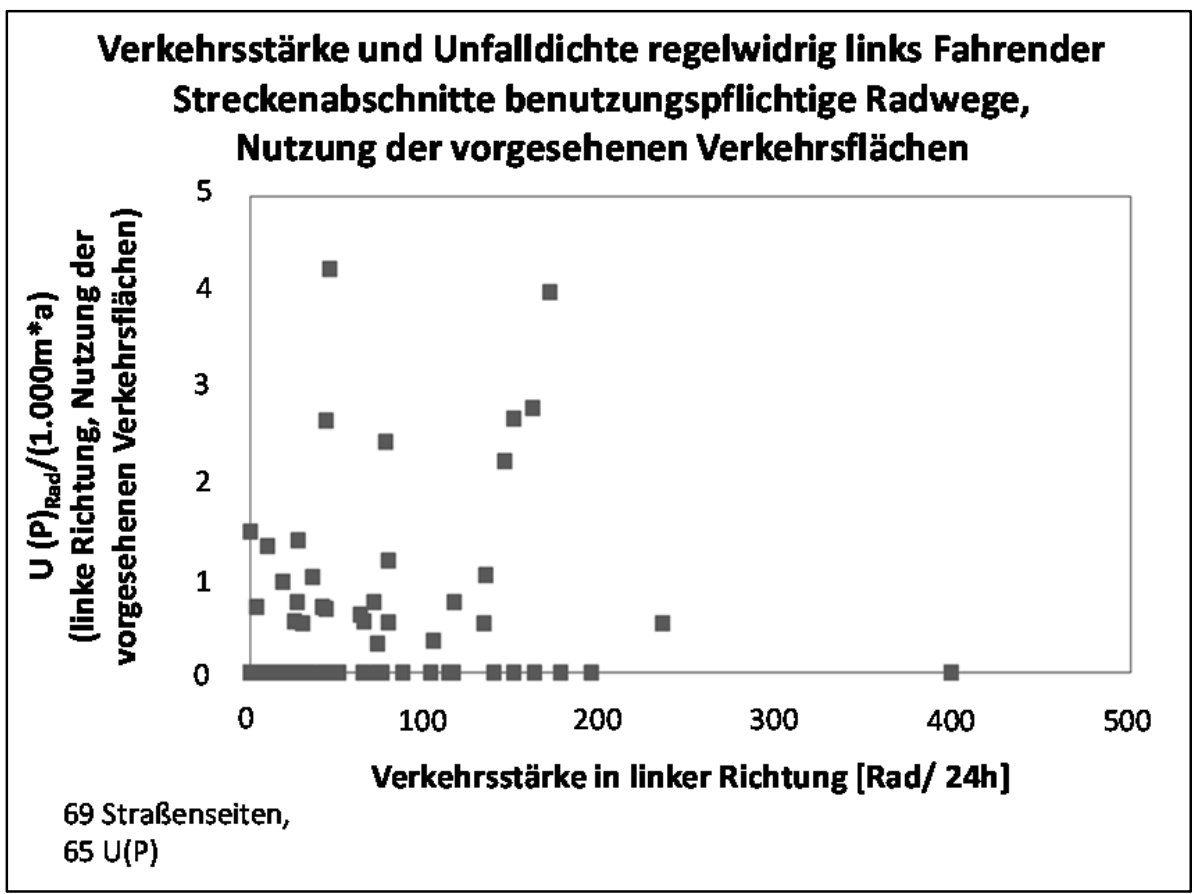

Bild 38: Anzahl regelwidrig links Fahrender auf Radweg und Unfalldichte links Fahrender auf Radweg in Straßen mit benutzungspflichtigen Radwegen (69 Straßenseiten, $65 \mathrm{U}(\mathrm{P})$ ) 
Für die Anzahl der Radfahrer in rechter Richtung und der Anzahl regelwidrig auf den Gehwegen Fahrender ergaben sich anhand der Auswertung der vierstündigen Zählungen vergleichsweise enge Zusammenhänge (Bild 39). Zwischen der Radverkehrsstärke in rechter Richtung und dem Anteil regelwidrig auf den Gehwegen Fahrenden konnten jedoch keine Zusammenhänge ermittelt werden (Bild 40). Eine verallgemeinerbare Aussage, dass die Radverkehrsstärken eine verstärkte anteilige Gehwegnutzung beeinflussen, ließ sich daher nicht ableiten.

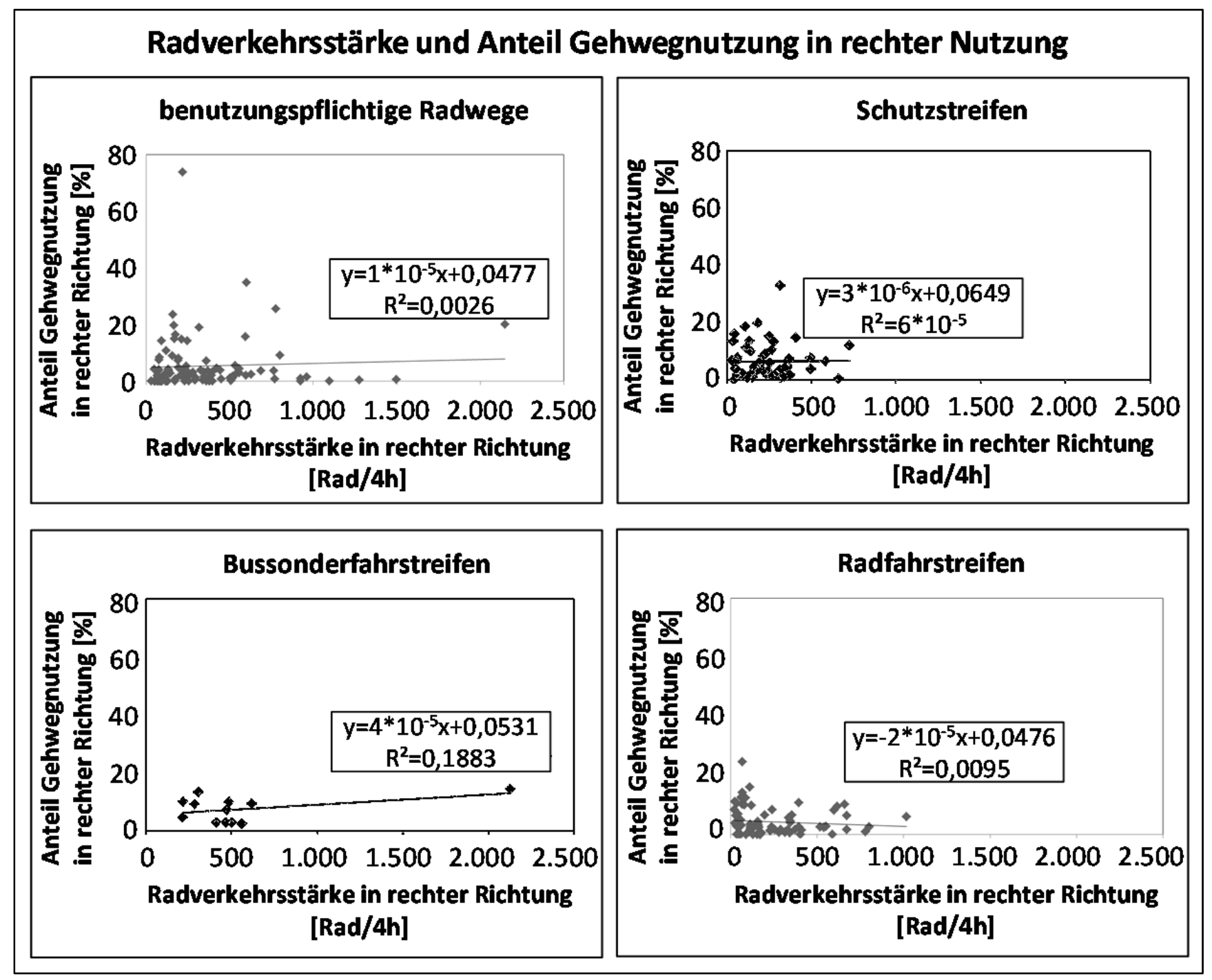

Bild 39: Radverkehrsstärke in rechter Richtung und Anzahl Gehwegnutzer in rechter Richtung 


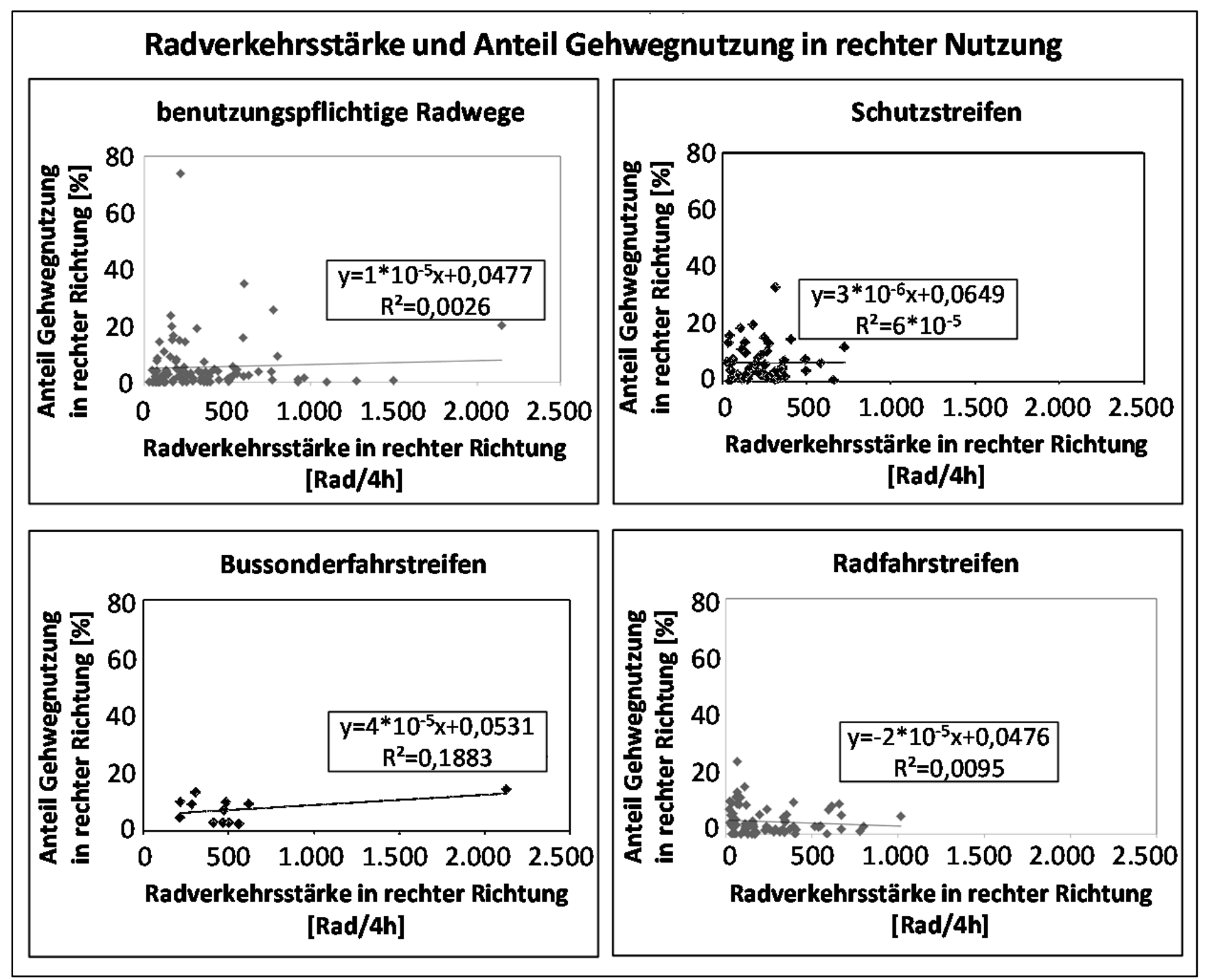

Bild 40:

Radverkehrsstärke in rechter Richtung und Anteil Gehwegnutzer in rechter Richtung 
In regelwidrig linker Fahrtrichtung fuhren die meisten Radfahrer bei Radwegen auf diesem selber und bei anderen Radverkehrsführungen auf den Gehwegen (Bild 41). Es wird vermutet, dass dies dem subjektiven Sicherheitsbedürfnis bei dieser Art von Regelübertretung geschuldet ist.

Über 90 \% der Radfahrer in rechter Fahrtrichtung nutzten bei den unterschiedlichen Anlagentypen die jeweils vorgesehene Radverkehrsführung. In Straßen mit Radwegen oder Schutzstreifen allerdings fuhren sechs bis acht Prozent der Radfahrer regelwidrig auf den Gehwegen, bei für den Radverkehr freigegebenen Bussonderfahrstreifen sogar fast ein Zehntel der Radfahrer.

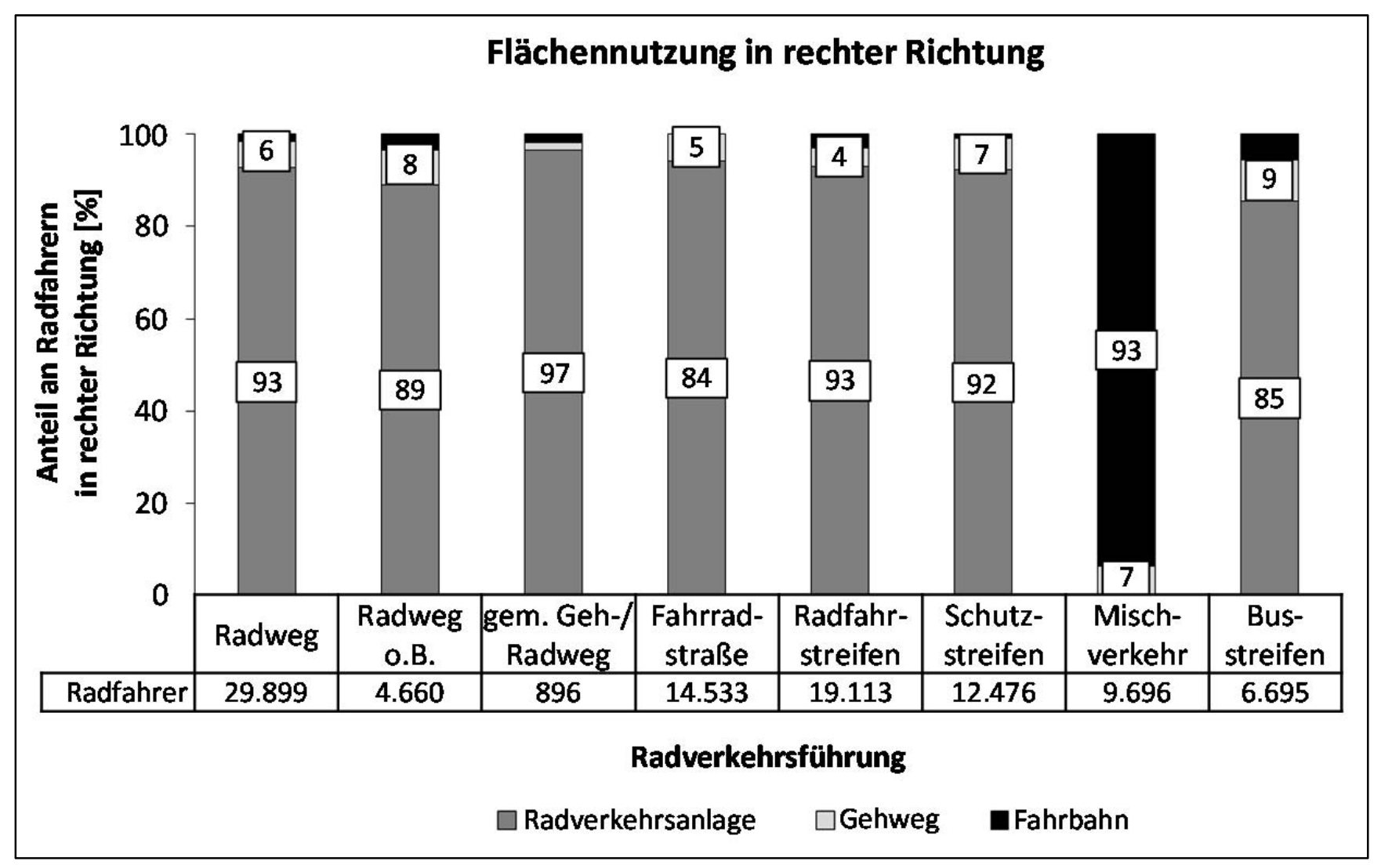

Bild 41: Flächennutzung in rechter Fahrtrichtung

\section{Flächennutzung und regelwidriges Linksfahren in Groningen (Exkurs)}

In der Untersuchung wurden über die deutschen Städte hinaus Verhaltensbeobachtungen in Groningen (Niederlande) durchgeführt. Bei den dort betrachteten 14 Radwegen, 4 Radfahrstreifen und 10 Schutzstreifen (Straßenseiten) zeigten sich mit etwa $1 \%$ sehr niedrige Anteile regelwidrig links Fahrender, auch in Straßen mit Einzelhandelsnutzungen. Auf den deutschen Streckenabschnitten lag dieser Anteil dagegen bei den verschiedenen Führungsformen zwischen 5 und $15 \%$. Bei den deutschen Untersuchungsabschnitten mit Radwegen zeigte sich, dass der Anteil regelwidrig links Fahrender mit steigender Radverkehrsstärke abnahm. Dieser Einfluss konnte anhand der niederländischen Untersuchungsabschnitte mit hohen Radverkehrsstärken bestätigt werden (Bild 42 und Bild 43). Allerdings zeigten sich auch in Groningen, bei mittleren Radverkehrsstärken um 500 Rad/4 Std., sehr niedrige Anteile regelwidrig links Fahrender. Es wird vermutet, dass die in den Niederlanden übliche Höhentrennung zwischen Rad- und Gehwegen dem Linksfahren entgegenwirkt, da links Fahrende bei Begegnungen mit Rechtsfahrenden nicht auf den Gehweg ausweichen können (Bild 44).

Im Unterschied zu den deutschen Städten nutzten die Groninger Radfahrer fast ausschließlich die Radverkehrsanlagen selber, eine Gehwegnutzung wurde auch in Straßen mit Radwegen kaum beobachtet. Es wird vermutet, dass auch dies durch die Höhentrennung beeinflusst ist (Bild 45). 


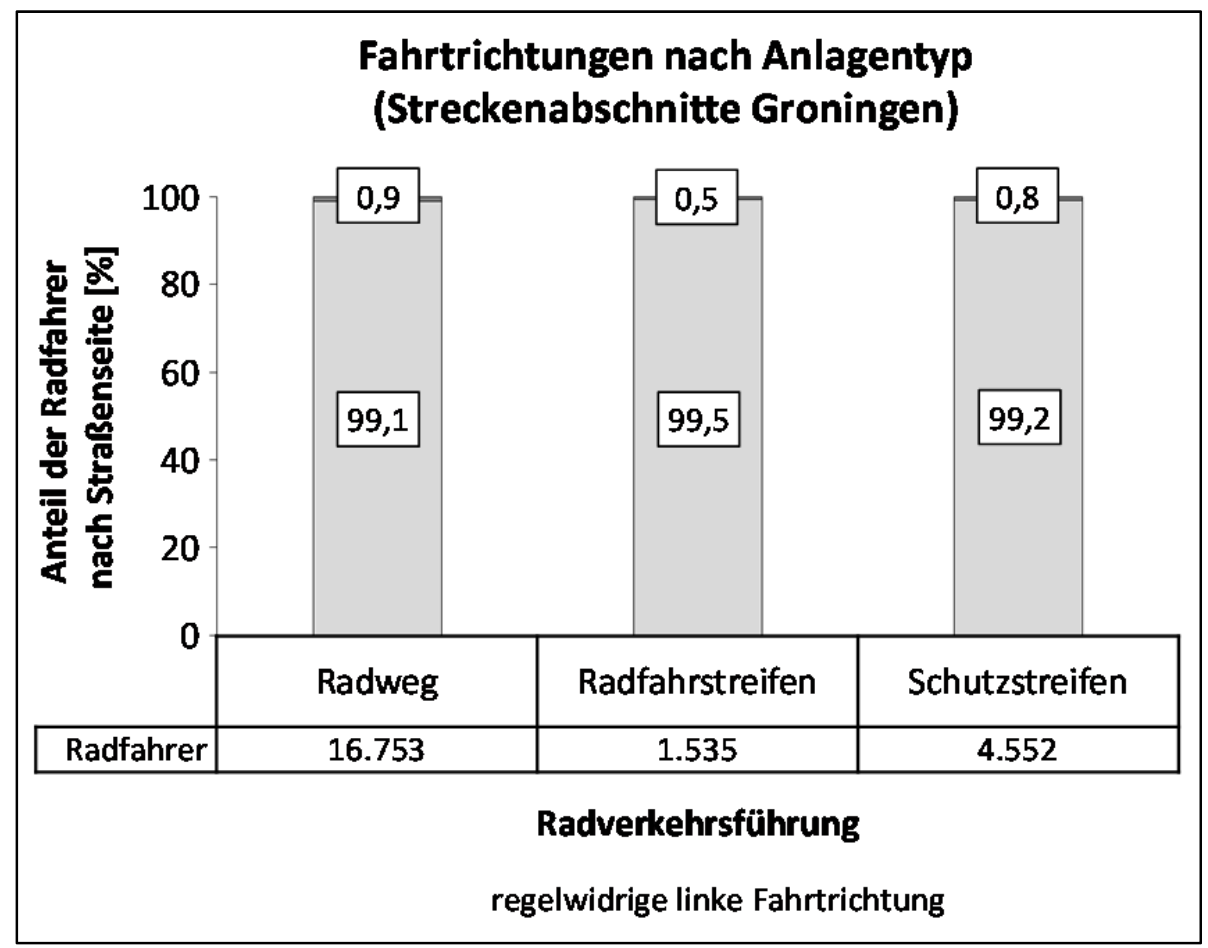

Bild 42: Anteile der Fahrtrichtungen auf den Groninger Streckenabschnitten

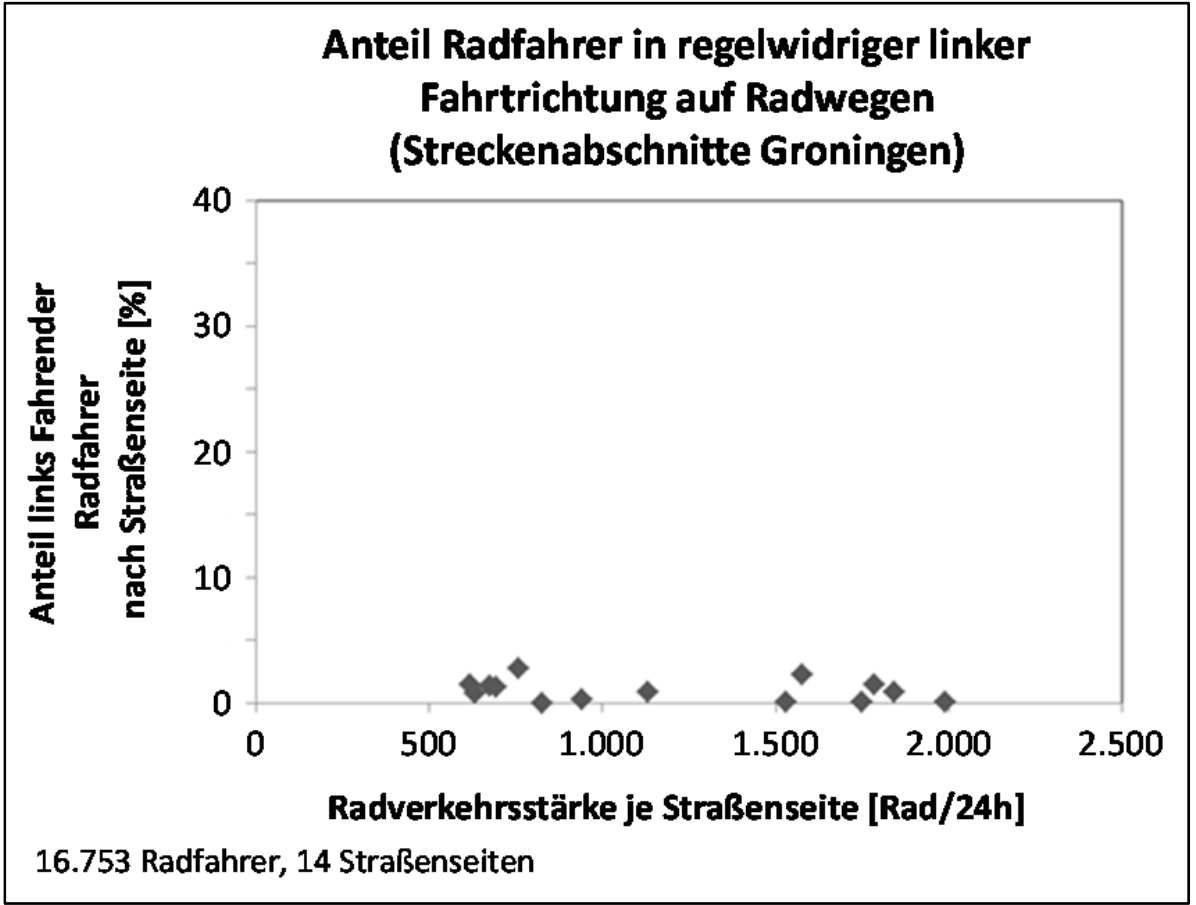

Bild 43: Anzahl Radfahrer und Anteil regelwidrig links Fahrender auf benutzungspflichtigen Radwegen in Groningen 


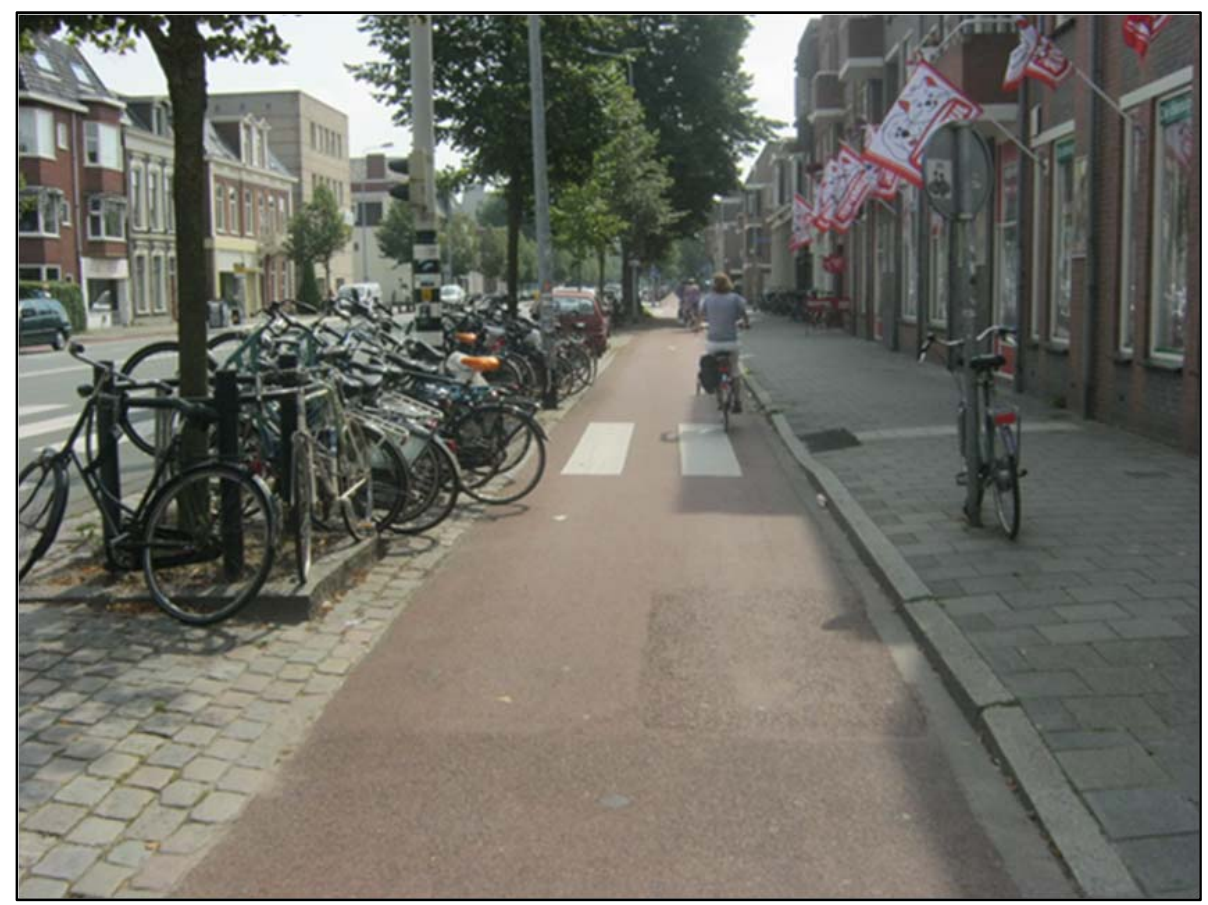

Bild 44: benutzungspflichtiger Radweg mit Höhentrennung zu Gehweg in Groningen

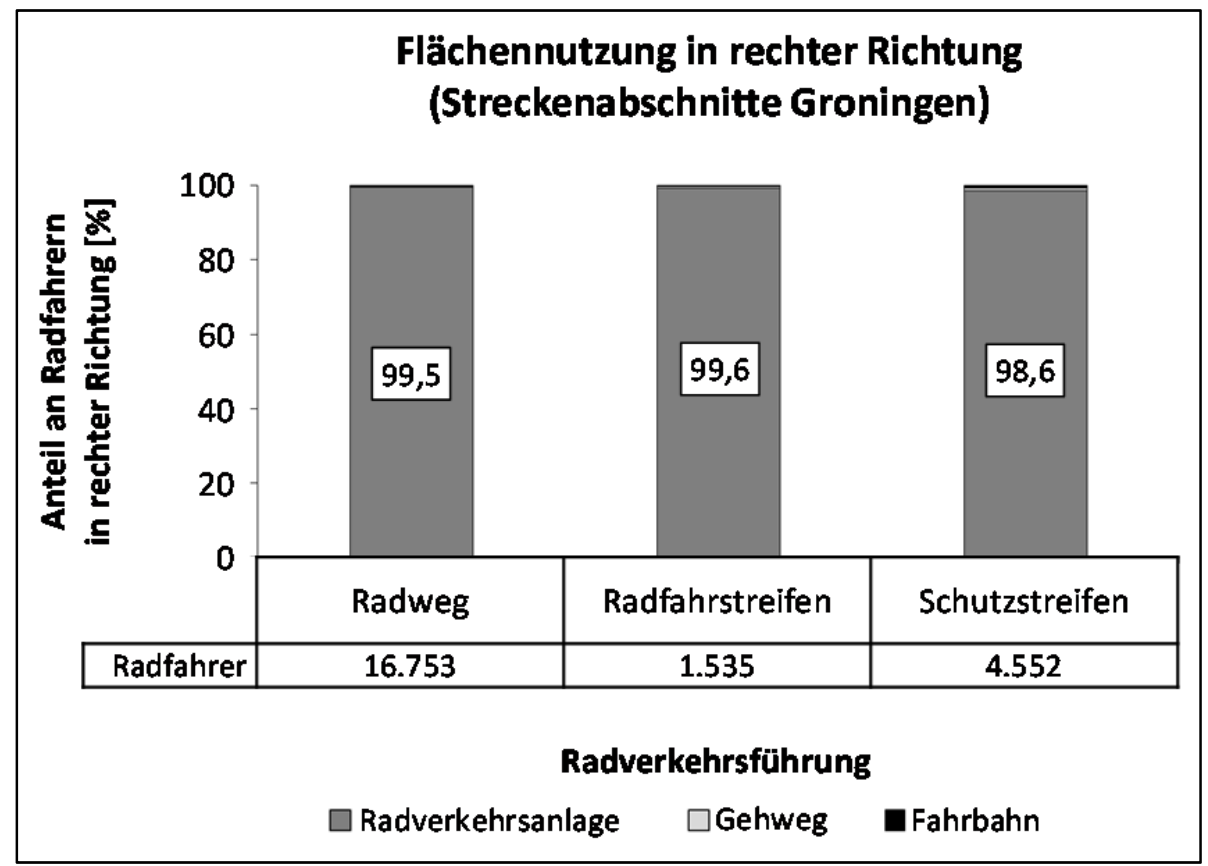

Bild 45: Flächennutzung in rechter Richtung auf den Groninger Untersuchungsabschnitten 


\section{Lichtsignalgeregelte Knotenpunkte}

In der Zusammenfassung aller Führungsformen zeigte sich aus der Mikroanalyse und den Verhaltensbeobachtungen kein erkennbarer Zusammenhang zwischen der Radverkehrsstärke und dem Anteil der Rotlichtübertretungen (Bild 46) ${ }^{30}$ Schon an Knotenpunkten mit nur vereinzelten Rotlichtübertretungen konnten dabei Rotfahrer-Unfälle auftreten. Auch im Rahmen der Makroanalyse ließen sich keine verallgemeinerbaren Zusammenhänge zwischen dem Anteil beobachteter Rotverstöße an allen Querungen vor Ort und dem Anteil der Ursache 31 „Nichtbeachten der Verkehrsregelung durch Polizeibeamte oder Lichtzeichen" an den Radverkehrsunfällen feststellen (Bild 47).

Anhand der Unfalldaten konnte nur das regelwidrige Fahren auf der Furt festgestellt werden, aber nicht eindeutig den Zufahrten zugeordnet werden. Eine eindeutige Gegenüberstellung der linksfahrenden Radfahrer und den Unfällen mit dieser Unfallursache war daher nicht möglich.

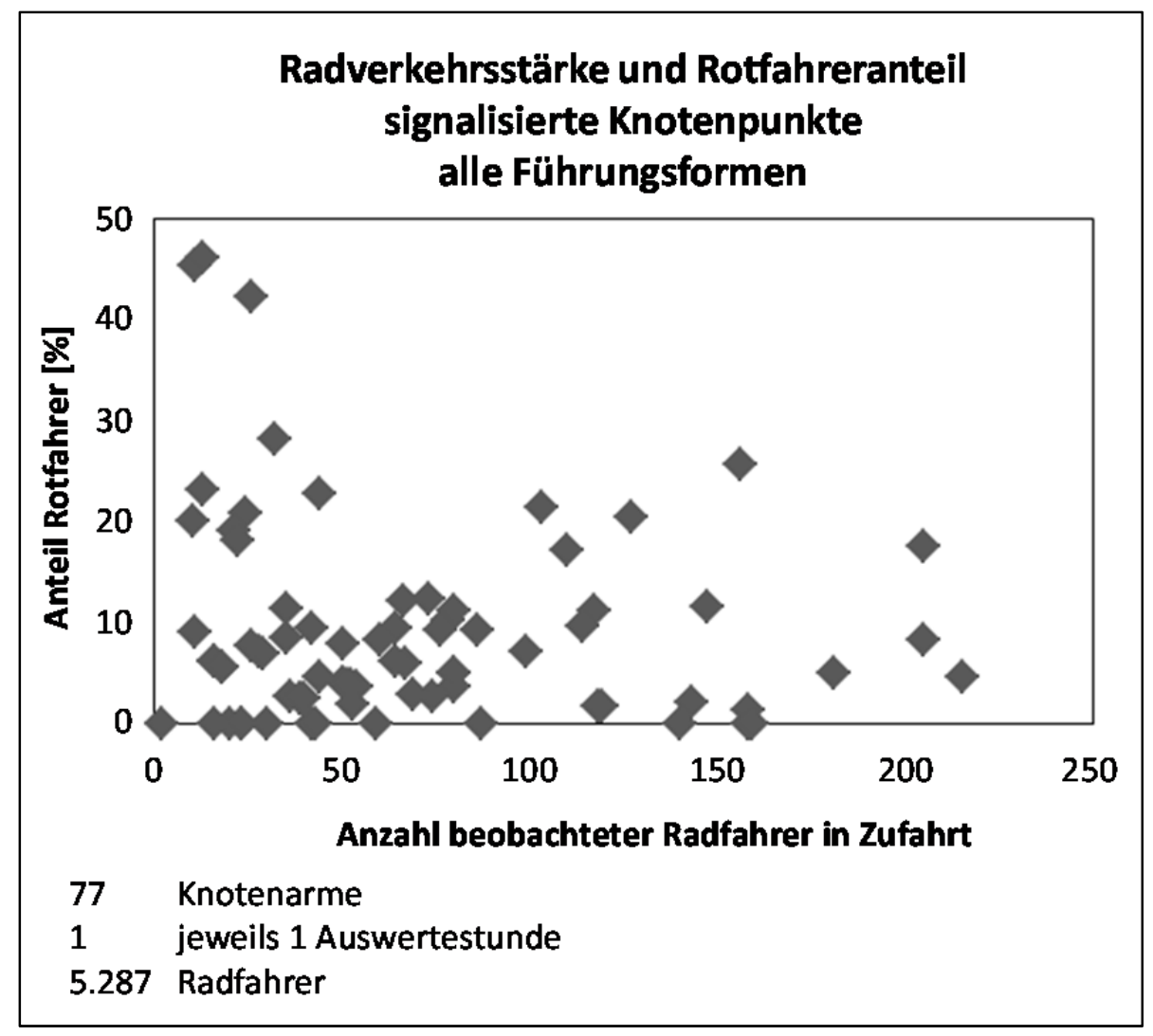

Bild 46: Anzahl beobachteter Radfahrer in der Zufahrt und Anteil der Rotfahrer bei Führung auf Fahrbahnen, Radwegen, Radfahrstreifen und Schutzstreifen 


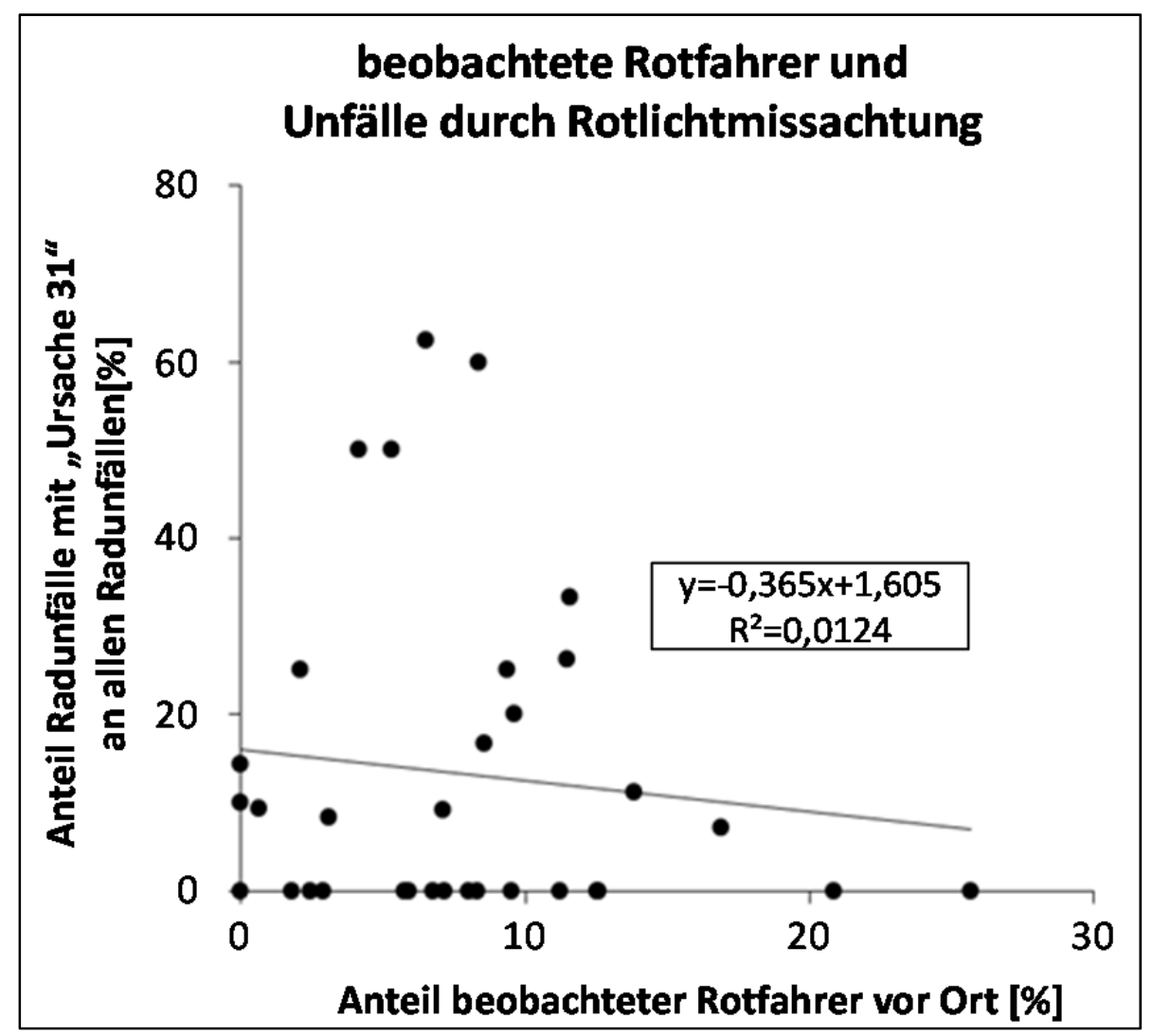

Bild 47: Anzahl beobachteter Radfahrer in der Zufahrt und Anteil der Unfälle in der Zufahrt mit „Nichtbeachten der Verkehrsregelung durch Lichtzeichen" als Unfallursache

\subsubsection{Zusammenfassung}

Zusammenfassend ließen sich die folgenden Einflüsse der Radverkehrsstärke auf das Unfallgeschehen und die Regelbefolgung festhalten.

An Streckenabschnitten mit Anschlussknotenpunkte bedingten höhere Radverkehrsstärken eine höhere Unfallanzahl. An lichtsignalgeregelten Knotenpunkten konnten schon bei niedrigen Radverkehrsstärken hohe Unfallbelastungen auftreten, ein Zusammenhang zwischen Radverkehrsstärke und Unfallanzahl war hier nicht erkennbar.

An Streckenabschnitten zeigten sich mit höheren Radverkehrsstärken höhere Anteile von Unfällen mit schwerem Personenschaden. An lichtsignalgeregelten Knotenpunkten konnten schon bei niedrigen Radverkehrsstärken hohe Anteile schwerer Unfälle auftreten. Ein verallgemeinerbarer Zusammenhang zwischen höheren Radverkehrsstärken und höherer Unfallschwere konnte an lichtsignalgeregelten Knotenpunkten nicht ermittelt werden.

Bei höheren Radverkehrsstärken verschoben sich die Unfalltypen an Streckenabschnitten zu Unfällen im Längsverkehr. Bei Radwegführung ereigneten sich bei höheren Radverkehrsstärken mehr Unfälle unter begegnenden Radfahrern auf dem Radweg. An lichtsignalgeregelten Knotenpunkten zeigten sich unabhängig von den Radverkehrsstärken bei unterschiedlichen Führungsformen typische Unfälle.

Sicherheitsprobleme durch das regelwidrige Linksfahren bestanden auf Streckenabschnitten vor allem bei Führung des Radverkehrs auf Radwegen an den Anschlussknotenpunkten. Die Anteile regelwidrig linksfahrender Radfahrer sanken bei höheren Radverkehrsstärken. Bereits bei geringen Stärken des regelwidrigen linken Radverkehrs waren jedoch bereits auffällig hohe Unfalldichten regelwidrig links Fahrender möglich. 


\subsection{Alter der Radfahrer, Unfallgeschehen und Regelbefolgung}

Vor dem Hintergrund bisheriger Erkenntnisse wurde vermutet, dass das Alter von Radfahrern Einfluss auf das zukünftige Unfallgeschehen und Verkehrsverhalten hat. Im Folgenden wurden daher Erkenntnisse aus den einzelnen Untersuchungsschritten zur Wirkung der Altersstruktur des Radverkehrs auf die Unfallanzahl, die Unfallschwere, die Unfalltypenverteilung und die Regelbefolgung zusammengefasst. Dabei wurde wiederum jeweils nach Streckenabschnitten und lichtsignalgeregelten Knotenpunkten differenziert.

\subsection{1. $\quad$ Alter und Unfallanzahl}

\section{Streckenabschnitte}

Zur Beschreibung des Alterseinflusses auf die Anzahl der Unfälle mit Radverkehrsbeteiligung wurden in der Makroanalyse die Unfallraten für Radfahrer verschiedener Altersgruppen bestimmt. Für die altersgruppenspezifische Radverkehrsstärke wurde der vor Ort erhobene Anteil einer Altersgruppe an allen Radfahrern auf die hochgerechnete Radverkehrsstärke angewendet, da eine altersspezifische Verkehrsleistung mit örtlicher Zuordnung nicht verfügbar war. Die Radverkehrsunfälle wurden den Altersgruppen anhand der Angaben in der Verkehrsunfallanzeige zum Alter des beteiligten Radfahrers zugeordnet.

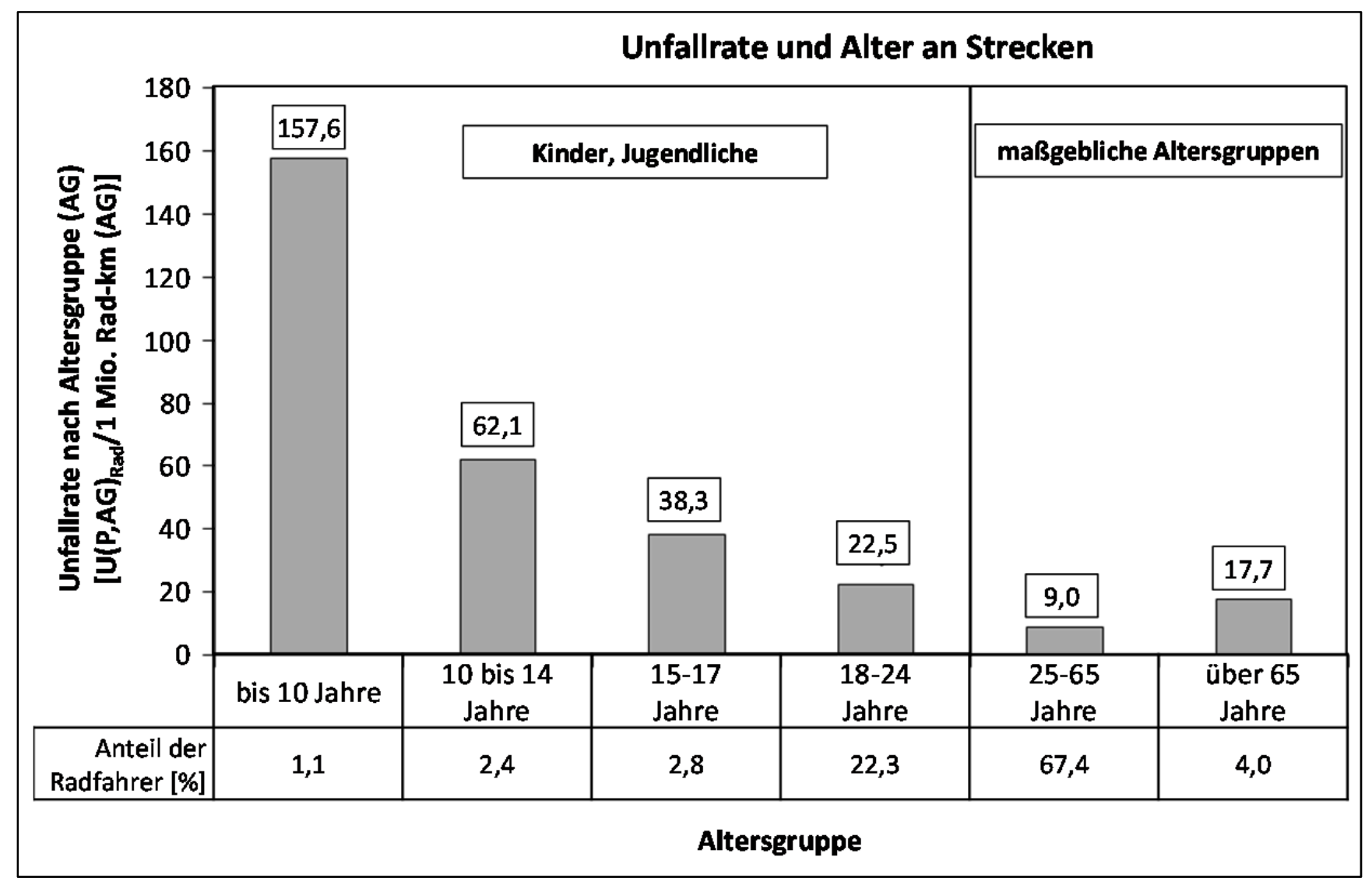

Bild 48: Unfallrate und Verkehrsbeteiligung auf den untersuchten Streckenabschnitten nach Altersgruppe der Radfahrer

Bild 48 zeigt, dass die Altersgruppe der 25- bis 65-jährigen Radfahrer die niedrigste Unfallrate aufwies. Etwa doppelt so hoch war die Unfallrate der Über-65-Jährigen. Sie lag damit etwas unter dem Niveau der Radfahrer zwischen 18-24 Jahren. Die höchsten Unfallraten zeigten sich bei Jugendlichen und Kindern.

Zur Bewertung des Einflusses des demografischen Wandels auf die Gesamtanzahl der Unfälle musste der Anteil der jeweiligen Altersgruppe an allen Radfahrern beachtet werden. Nach den hier vorliegenden Zählergebnissen bildeten Jugendliche und Über-65-Jährige nur ca. 10 \% des Radfahrerkol- 
lektivs. Die Unfallanzahl und das Radverkehrsaufkommen waren im Wesentlichen geprägt von beteiligten Radfahrern im Alter zwischen 18-65 Jahren. ${ }^{31}$

\section{Lichtsignalgeregelte Knotenpunkte}

Nach der Mikroanalyse betrug der Anteil der Unfälle von Über-65-Jährigen an allen RadfahrerUnfällen an Knotenarmen mit bekannten Radverkehrsstärken der zuführenden Streckenabschnitte 7,1\%. Nach den Verhaltensbeobachtungen lag der Anteil von Über-65-Jährigen am Radverkehr der zuführenden Streckenabschnitte mit 4,6\% niedriger. Die insgesamt wenigen Unfälle der Über-65Jährigen (nur 12 Unfälle) schränkten die Verlässlichkeit einer verallgemeinerbaren Aussage jedoch ein.

An den meisten untersuchten Knotenarmen ereigneten sich keine Unfälle mit Über-65-Jährigen. An den Knotenarmen, an denen Über-65-Jährige nach Bild 49 an $50 \%$ oder $100 \%$ aller Radfahrerunfälle beteiligt waren, ereigneten sich nur ein oder zwei Unfälle mit Personenschaden in drei Jahren.

Insgesamt konnte damit bei steigenden Anteilen von Über-65-Jährigen am Radverkehr kein verallgemeinerbarer Anstieg der Unfallzahlen an lichtsignalgeregelten Knotenpunkten festgestellt werden.

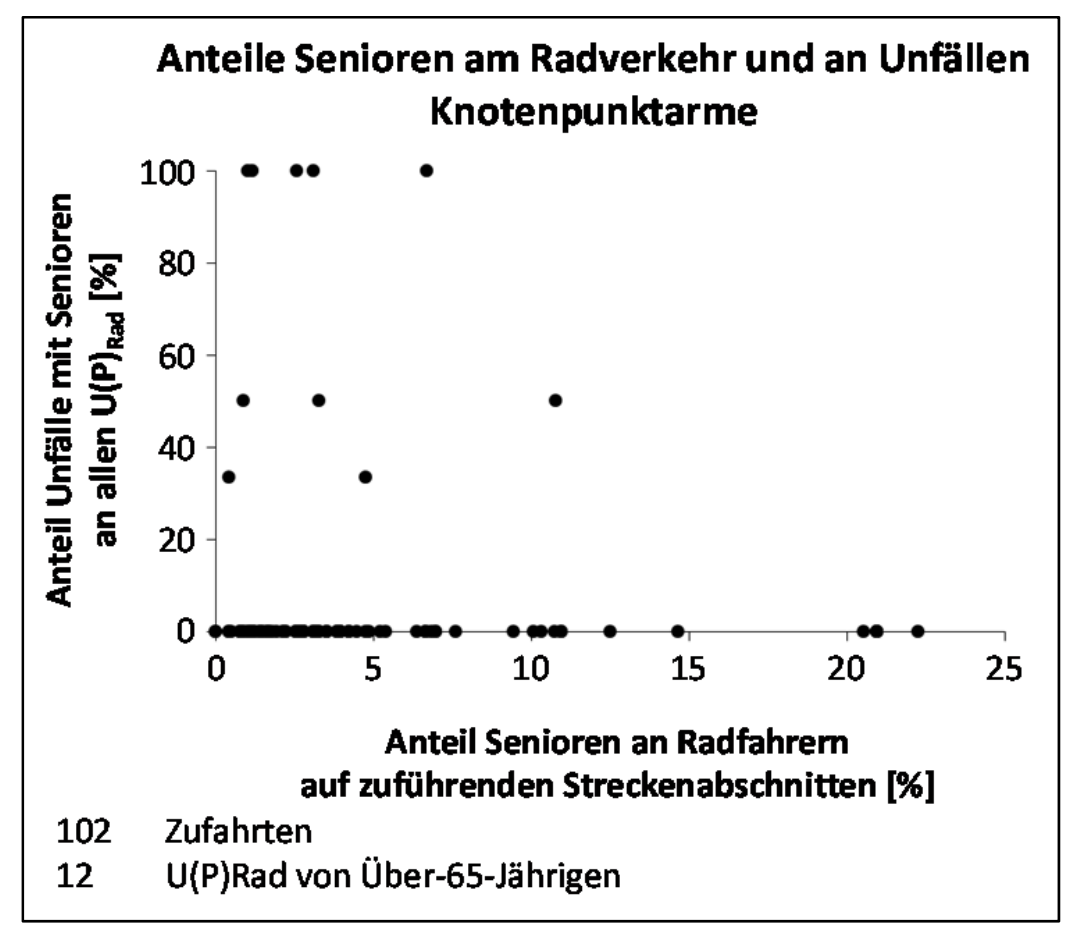

Bild 49: Anteile der Unfälle mit Über-65-Jährigen und Anteil der Über-65-Jährigen am Radverkehr auf zuführendem Streckenabschnitt 


\subsubsection{Alter und Unfallschwere}

\section{Streckenabschnitte}

In der Makro- und in der Mikroanalyse wurden nur vergleichsweise wenige Unfälle Über-65-Jähriger erfasst. Um ein größeres Unfallkollektiv auswerten zu können, wurde in der Makroanalyse zur Ermittlung der Wirkung des Alters auf die Unfallschwere das Gesamtunfallgeschehen der Radfahrer (Unfälle mit Personenschaden) derjenigen Untersuchungsstädte herangezogen, von denen auch über die Untersuchungsstrecken hinaus Unfalldaten der Jahre 2009-2011 vorlagen ${ }^{32}$. Anhand der Charakteristik der Unfallstelle und den Angaben zur Verkehrsregelung (Lichtzeichenanlage) wurden die Radverkehrsunfälle in Knotenpunkt- und Streckenunfälle eingeordnet ${ }^{33}$.

Die Beschreibung des Einflusses des Alters auf die Unfallschwere erfolgte über die Einteilung der Unfälle nach dem Alter der beteiligten Radfahrer (unabhängig von der Unfallverursachung über die Ordnungsnummer) und der Zuordnung der Unfallkategorien auf die zwei Altersklassen. Als Grenze der Alterszuordnung galt ein Alter von 65 Jahren des Radfahrers. Da je nach Unfallverursachung Unterschiede in der Unfallschwere auftreten konnten, wurde in Bild 50 und Bild 51 die Unfallstruktur getrennt für das gesamte Radverkehrsunfallgeschehen (linke Seite) und für Unfälle mit dem Radfahrer als Hauptverursacher (rechte Seite) dargestellt.

33 Es wurden nur Rad-Unfälle mit Personenschaden ausgewertet mit der Ortslage „innerorts“. Für Streckenunfälle durften die Merkmale „LSA an“ und „LSA aus“ nicht enthalten sein; für Knotenpunktunfälle mussten die Merkmale „Einmündung“ oder „Kreuzung“ enthalten sein und Angaben zur Lichtzeichenanlage vorliegen (,in Betrieb “ oder außer Betrieb"). Damit entsprach die Streckendefinition derjenigen in der Untersuchung an anderer Stelle, da Unfälle an Knotenpunkten ohne LSA (Anschlussknoten) zu den Streckenunfällen gezählt wurden. 


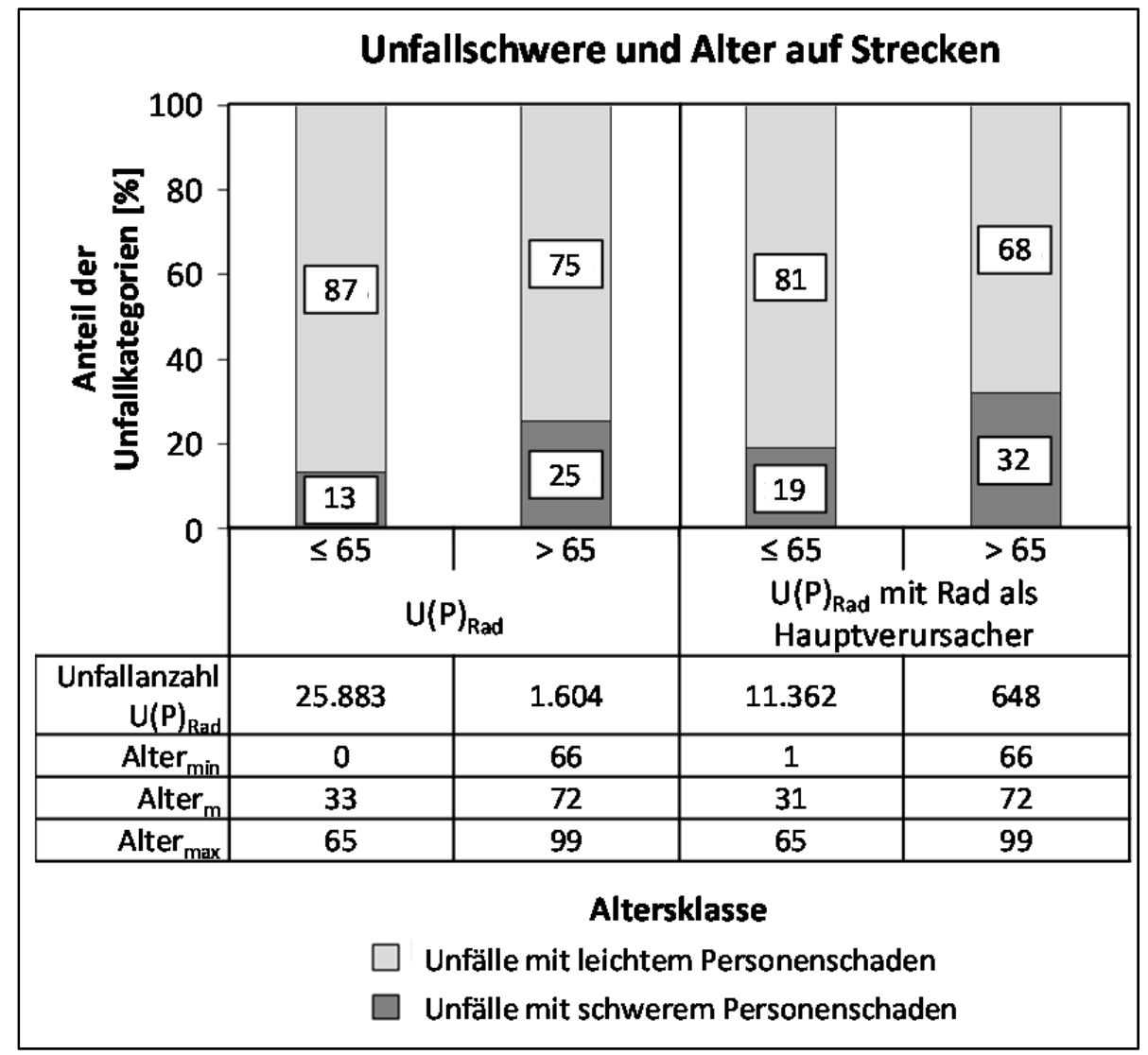

Bild 50: Unfallschwere nach Alter der Radfahrer und Verursachung auf Streckenabschnitten (erweitertes Unfallkollektiv) ${ }^{33}$

Bei Streckenunfällen mit Radverkehrsbeteiligung lag unabhängig von der Unfallverursachung der Anteil der Unfälle mit schwerem Personenschaden in der Altersklasse der Über-65-Jährigen mit $25 \%$ deutlich höher als bei jüngeren beteiligten Rad Fahrenden mit $13 \%$.

War ein Radfahrer der Hauptunfallverursacher, dann stieg der Anteil der Unfälle mit schwerem Personenschaden in den zwei Altersklassen generell um etwa sechs bis sieben Prozentpunkte. Die Unterschiede der Unfallschwere blieben bei Betrachtung der zwei Altersklassen in gleicher Größenordnung erhalten. 


\section{Lichtsignalgeregelte Knotenpunkte}

Ähnliche Ergebnisse zeigte die Auswertung der Radverkehrsunfälle an lichtsignalgeregelten Knotenpunkten für das erweiterte Unfallkollektiv (Bild 51). Im Gesamtunfallgeschehen der Radfahrer lag der Anteil der Unfälle mit schwerem Personenschaden in der Altersklasse der Unter-65-Jährigen bei $14 \%$; in der Klasse der Über-65-Jährigen bei $27 \%$. Wurden hier nur die Unfälle mit dem Rad als Hauptverursacher betrachtet, erhöhten sich die Werte um sechs bis acht Prozentpunkte.

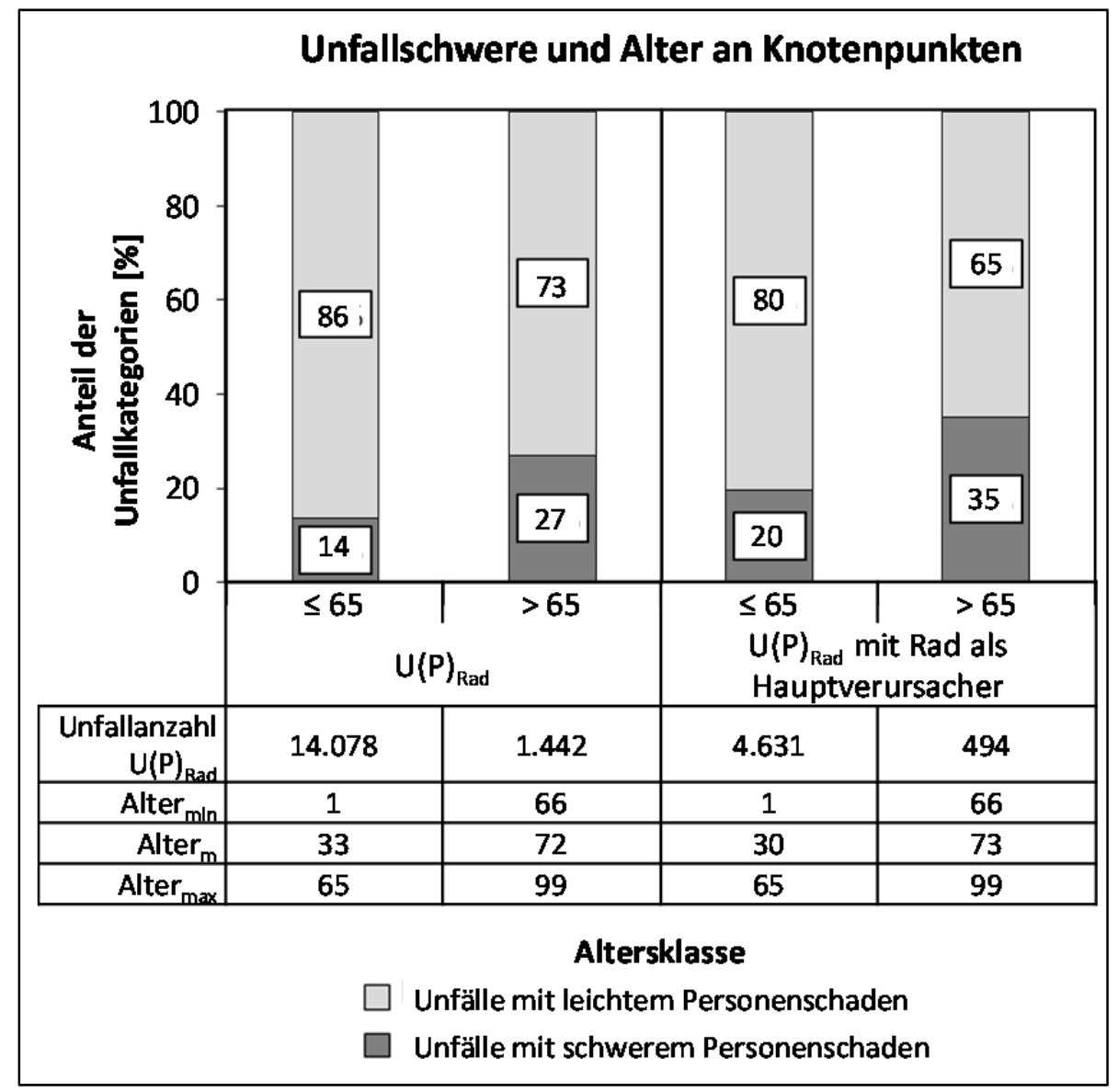

Bild 51: Unfallschwere nach Alter der Radfahrer und Verursachung an Verkehrsknotenpunkten mit LSA (erweitertes Unfallkollektiv) ${ }^{33}$ 


\subsubsection{Alter und Unfalltypenverteilung}

\section{Streckenabschnitte}

Die Datenbasis und die Aufteilung nach Knoten- und Streckenunfällen und den Altersklassen entsprach in der Makroanalyse dem Vorgehen wie im Abschnitt 4.3.2. Um Verschiebungen in den Anteilen der relevanten Unfalltypen zu verdeutlichen, wurden Unfälle des Typs 7 „Sonstige“ nicht mit betrachtet $^{34}$.

Im Gesamtunfallgeschehen mit Radverkehrsbeteiligung des erweiterten Unfallkollektivs ließen sich zwischen den zwei Altersklassen nur geringe Unterschiede in der Verteilung der Unfalltypen erkennen. Wurden nur die Unfälle mit Radfahrern als Hauptverursacher betrachtet, dann fielen im Vergleich zum Gesamtunfallgeschehen besonders viele Fahrunfälle auf. Gegenüber der Altersklasse der Über65-Jährigen (40 \%) lag der Anteil der Fahrunfälle für die Altersklasse der Unter-65-Jährigen jedoch auf einem niedrigeren Niveau (33\%) (Bild 52).

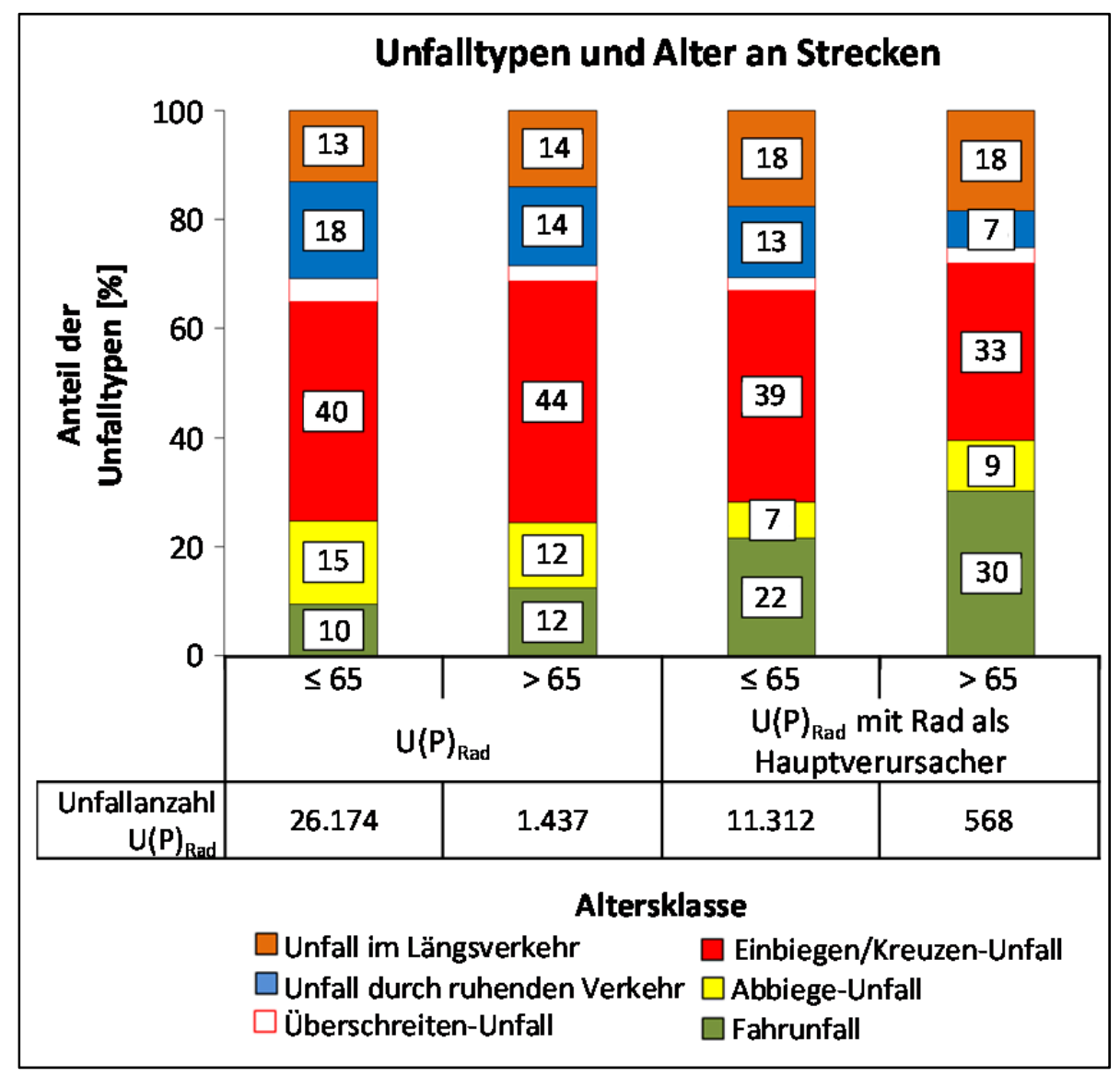

Bild 52: Unfalltypen (Anteile, ohne Typ 7) nach Alter und Unfallverursachung an Strecken (erweitertes Unfallkollektiv) ${ }^{33}$ 


\section{Lichtsignalgeregelte Knotenpunkte}

An lichtsignalgeregelten Knotenpunkten ließen sich bereits im Gesamtunfallgeschehen des erweiterten Fahrradunfallkollektivs Unterschiede zwischen den Altersklassen feststellen (Bild 53). Um Verschiebungen in den Anteilen der relevanten Unfalltypen in der Makroanalyse zu verdeutlichen, wurden Unfälle des Typs 7 „Sonstige“ nicht mit betrachtet. ${ }^{35}$ Die Einbiegen-/ Kreuzen-Unfälle nahmen in der Altersklasse der Über-65-Jährigen den größten Anteil ein. In der Altersklasse der Unter-65Jährigen war der Abbiege-Unfall stärker ausgeprägt. Bei den Unfällen mit Radfahrern als Hauptverursacher trat dieser Unterschied zwischen den Altersklassen nicht auf. Dafür zeigte sich bei den durch Radfahrer verursachten Unfällen ein höherer Anteil von Längsverkehrsunfällen in der Altersklasse der Unter-65-Jährigen als in der Klasse der Über-65-Jährigen (Bild 53).

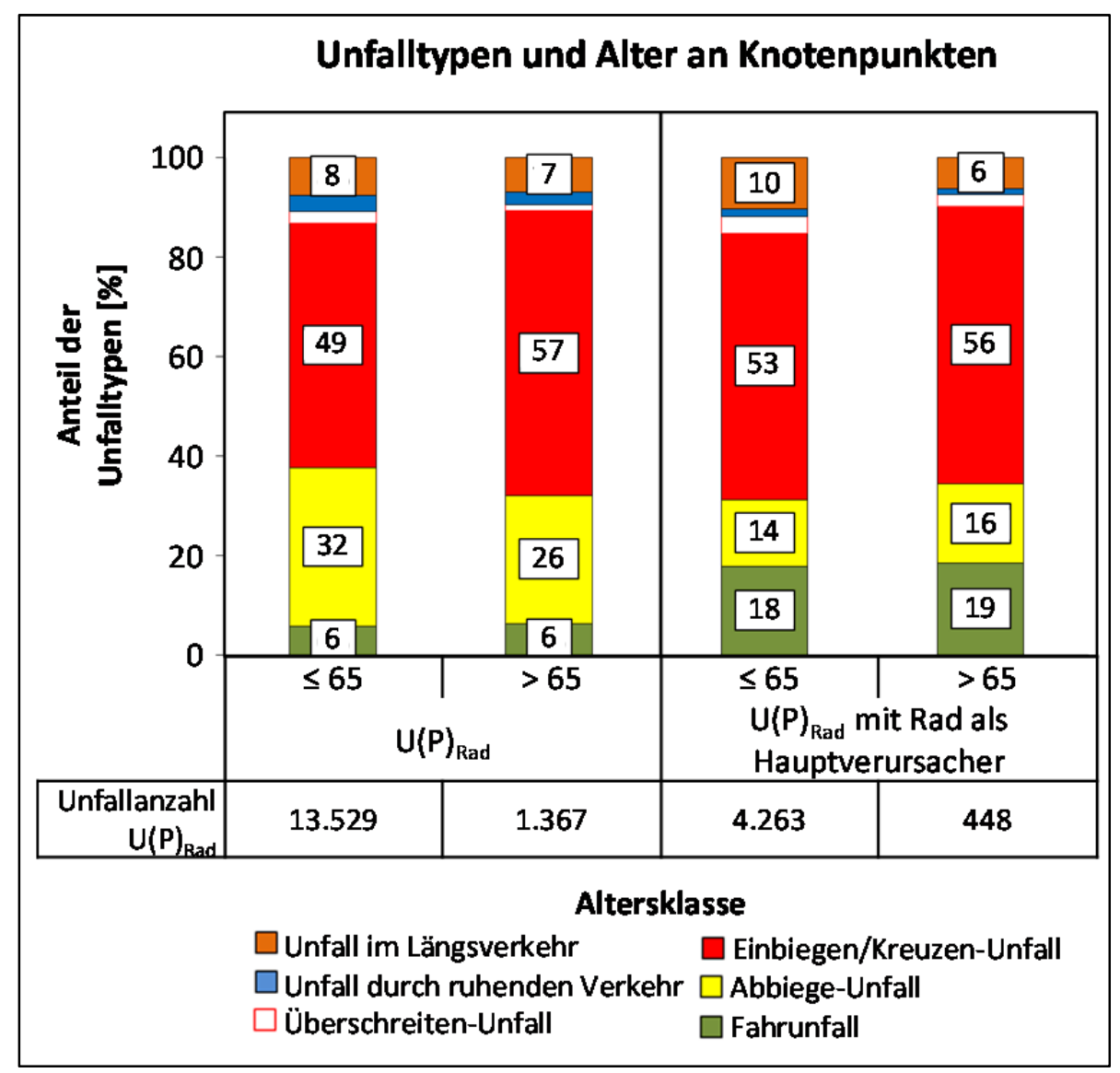

Bild 53: Unfalltypen (Anteile, ohne Typ 7) nach Alter und Unfallverursacher an Knotenpunkten (erweitertes Unfallkollektiv) ${ }^{33}$ 


\subsubsection{Alter und Regelbefolgung}

\section{Streckenabschnitte}

Nach den Verhaltensbeobachtungen fuhren $9 \%$ der Über-65-Jährigen in regelwidrig linker Fahrtrichtung. Dieser Anteil lag mit 1,3 Prozentpunkten geringfügig höher als bei den 18- bis 24- und den 25bis 65-Jährigen (Tabelle 29).

Auf den Streckenabschnitten, die auch für die Mikroanalyse der Unfälle herangezogen wurden, fuhren fast $12 \%$ der Über-65-Jährige in regelwidriger linker Richtung (Tabelle 30). Fast ein Viertel der Unfälle von Über-65-Jährigen ereigneten sich in regelwidriger linker Fahrtrichtung. Vergleichbar häufig verunglückten 18- bis 24-Jährige in linker Fahrtrichtung. 25- bis 65-Jährige verunglückten bei etwa einem Fünftel der Unfälle dieser Altersgruppe in linker Richtung.

Tabelle 29: Beobachtete Fahrtrichtungen (alle Streckenabschnitte)

\begin{tabular}{|l|c|c|c|}
\hline \multirow{2}{*}{} & \multicolumn{2}{|c|}{ Anteile Fahrtrichtung [\%] } & \\
\cline { 2 - 4 } & regelwidrig links & rechts & beobachtete Radfahrer \\
\hline $\mathbf{1 0}$ J. & 25,4 & 74,6 & 1.226 \\
\hline $\mathbf{1 0 - 1 4}$ J. & 15,4 & 84,6 & 2.559 \\
\hline $\mathbf{1 5 - 1 7}$ J. & 7,1 & 92,9 & 3.057 \\
\hline $\mathbf{1 8 - 2 4}$ J. & 7,7 & 92,3 & 24.236 \\
\hline $\mathbf{2 5 - 6 5}$ J. & 7,7 & 92,3 & 73.226 \\
\hline >65 J. & 9,0 & 91,0 & 4.357 \\
\hline
\end{tabular}

Tabelle 30: Beobachtete Fahrtrichtungen und Fahrtrichtungen (Streckenabschnitte der Mikroanalyse)

\begin{tabular}{|c|c|c|c|c|c|c|c|c|}
\hline & \multicolumn{2}{|c|}{ Anteile Fahrtrichtung [\%] } & \multirow{2}{*}{$\begin{array}{l}\text { Anzahl beobachteter } \\
\text { Radfahrer }\end{array}$} & \multicolumn{4}{|c|}{$\begin{array}{c}\text { Fahrtrichtung bei } \\
\text { Unfällen mit Personenschaden [\%] }\end{array}$} & \multirow{2}{*}{$\begin{array}{c}\text { Anzahl Unfälle mit Perso- } \\
\text { nenschaden }\end{array}$} \\
\hline & $\begin{array}{c}\text { regelwidrig } \\
\text { links }\end{array}$ & rechts & & links & rechts & quert & k. A. & \\
\hline < $10 \mathrm{~J}$. & 28,5 & 71,5 & 575 & 16,7 & 33,3 & 50,0 & 0,0 & 6 \\
\hline 10-14 J. & 22,9 & 77,1 & 789 & 35,3 & 23,5 & 35,3 & 5,9 & 17 \\
\hline $15-17 \mathrm{~J}$. & 19,0 & 81,0 & 747 & 20,0 & 40,0 & 40,0 & 0,0 & 10 \\
\hline 18-24 J. & 9,8 & 90,2 & 12.374 & 24,5 & 58,2 & 11,2 & 6,1 & 98 \\
\hline 25-65 J. & 10,2 & 89,8 & 34.952 & 18,2 & 60,3 & 17,2 & 4,4 & 297 \\
\hline$>65 \mathrm{~J}$. & 11,9 & 88,1 & 2.252 & 23,3 & 43,3 & 30,0 & 3,3 & 30 \\
\hline
\end{tabular}

Über-65-Jährige nutzten ebenso wie Radfahrer im Alter von 18 bis 65 Jahren überwiegend die jeweiligen Radverkehrsanlagen. Auch bei Radfahrstreifen- oder Mischverkehrsführungen fuhren über $90 \%$ der Über-65-Jährigen auf der Fahrbahn bzw. dem Radfahrstreifen (Tabelle 31 bis Tabelle 34).

In Straßen mit Schutzstreifen fuhren Über-65-Jährige in rechter Richtung häufiger auf dem Gehweg als 25 - bis 65-Jährige (8\% gegenüber $6 \%$, Tabelle 35). Hier bestanden - ebenso wie bei der Gehwegnutzung in rechter Richtung in Straßen mit anderen Radverkehrsanlagen - nach den polizeilichen Daten aber keine besonderen Unfallgefährdungen.

Tabelle 31: Flächennutzung nach Fahrtrichtung und Altersgruppe bei benutzungspflichtigen Radwegen (Anteil nach Altersgruppe in \%)

\begin{tabular}{|c|c|c|c|c|c|c|c|c|c|c|c|c|}
\hline \multicolumn{13}{|c|}{ Benutzungspflichtige Radwege } \\
\hline & \multicolumn{12}{|c|}{ Anteil Fahrtrichtung und Alter [\%] } \\
\hline & links & \multicolumn{5}{|c|}{ regelwidrig links } & \multicolumn{6}{|c|}{ rechts } \\
\hline $\begin{array}{c}\text { genutzte Flä- } \\
\text { che }\end{array}$ & $<10 \mathrm{~J}$ & $10-14 \mathrm{~J}$. & $15-17 \mathrm{~J}$. & $18-24 \mathrm{~J}$. & $25-65 \mathrm{~J}$. & $>65 \mathrm{~J}$. & $<10 \mathrm{~J}$ & 10-14 J. & $15-17 \mathrm{~J}$. & 18-24 J. & $25-65 \mathrm{~J}$. & $>65 \mathrm{~J}$. \\
\hline $\begin{array}{l}\text { Radverkehrs- } \\
\text { anlage }\end{array}$ & 44,0 & 69,9 & 80,4 & 71,2 & 68,6 & 65,7 & 67,8 & 86,3 & 96,8 & 92,5 & 93,1 & 94,1 \\
\hline Gehweg & 54,9 & 30,1 & 17,4 & 28,0 & 31,2 & 33,7 & 31,5 & 13,1 & 2,9 & 5,8 & 5,1 & 4,9 \\
\hline Fahrbahn & - & - & - & 0,3 & 0,1 & - & 0,4 & 0,2 & 0,3 & 1,6 & 1,8 & 1,0 \\
\hline $\begin{array}{l}\text { andere Ver- } \\
\text { kehrsfläche }\end{array}$ & 1,1 & - & 2,2 & 0,4 & 0,1 & 0,6 & 0,4 & 0,4 & - & 0,1 & 0,1 & - \\
\hline Gesamt & 100,0 & 100,0 & 100,0 & 100,0 & 100,0 & 100,0 & 100,0 & 100,0 & 100,0 & 100,0 & 100,0 & 100,0 \\
\hline$N$ & 91 & 136 & 92 & 913 & 2.743 & 169 & 273 & 489 & 622 & 6.530 & 20.701 & 1.284 \\
\hline
\end{tabular}


Tabelle 32: Flächennutzung nach Fahrtrichtung und Altersgruppe bei nicht benutzungspflichtigen Radwegen (Anteil nach Altersgruppe in \%)

\begin{tabular}{|c|c|c|c|c|c|c|c|c|c|c|c|c|}
\hline \multicolumn{13}{|c|}{ Nicht benutzungspflichtige Radwege } \\
\hline & \multicolumn{12}{|c|}{ Anteil Fahrtrichtung und Alter [\%] } \\
\hline & links & \multicolumn{4}{|c|}{ regelwidrig links } & \multicolumn{7}{|c|}{ rechts } \\
\hline $\begin{array}{c}\text { genutzte Flä- } \\
\text { che }\end{array}$ & $<10 \mathrm{~J}$ & 10-14 J. & $15-17 \mathrm{~J}$. & 18-24 J. & $25-65 \mathrm{~J}$. & $>65 \mathrm{~J}$ & $<10 \mathrm{~J}$ & 10-14 J. & $15-17 \mathrm{~J}$. & $18-24 \mathrm{~J}$. & $25-65 \mathrm{~J}$. & $>65 \mathrm{~J}$. \\
\hline $\begin{array}{l}\text { Radverkehrs- } \\
\text { anlage }\end{array}$ & 85,7 & 80,4 & 78,3 & 78,8 & 76,8 & 75,4 & 71,9 & 82,6 & 86,8 & 92,3 & 89,0 & 91,4 \\
\hline Gehweg & 14,3 & 19,6 & 20,3 & 21,2 & 22,8 & 24,6 & 27,0 & 16,3 & 11,8 & 4,7 & 6,7 & 7,9 \\
\hline Fahrbahn & - & - & 1,4 & - & 0,4 & - & 1,1 & 0,6 & 1,3 & 3,0 & 4,3 & 0,7 \\
\hline $\begin{array}{l}\text { andere Ver- } \\
\text { kehrsfläche }\end{array}$ & - & - & - & - & - & - & - & 0,6 & - & - & 0,0 & - \\
\hline Gesamt & 100,0 & 100,0 & 100,0 & 100,0 & 100,0 & 100,0 & 100,0 & 100,0 & 100,0 & 100,0 & 100,0 & 100,0 \\
\hline $\mathbf{N}$ & 28 & 46 & 69 & 113 & 479 & 65 & 89 & 172 & 228 & 598 & 3131 & 442 \\
\hline
\end{tabular}

Tabelle 33: Flächennutzung nach (Anteil nach Altersgruppe in \%)

Fahrtrichtung und $\quad$ Altersgruppe

\begin{tabular}{|c|c|c|c|c|c|c|c|c|c|c|c|c|}
\hline \multicolumn{13}{|c|}{ Mischverkehr } \\
\hline \multirow[b]{3}{*}{$\begin{array}{l}\text { genutzte } \\
\text { Fläche }\end{array}$} & \multicolumn{12}{|c|}{ Anteil Fahrtrichtung und Alter [\%] } \\
\hline & \multirow{2}{*}{$\begin{array}{c}\text { links } \\
<10 \mathrm{~J} .\end{array}$} & \multicolumn{4}{|c|}{ regelwidrig links } & \multicolumn{7}{|c|}{ rechts } \\
\hline & & $10-14 \mathrm{~J}$. & $15-17 \mathrm{~J}$. & $18-24 \mathrm{~J}$. & $25-65 \mathrm{~J}$. & $>65 \mathrm{~J}$. & $<10 \mathrm{~J}$. & $10-14 \mathrm{~J}$. & $15-17 \mathrm{~J}$. & $18-24 \mathrm{~J}$. & $25-65 \mathrm{~J}$. & $>65 \mathrm{~J}$. \\
\hline Gehweg & 97,7 & 100,0 & 100,0 & 98,6 & 97,8 & 100,0 & 67,1 & 45,2 & 10,0 & 5,5 & 5,5 & 6,5 \\
\hline Fahrbahn & - & - & - & - & - & - & 31,7 & 54,8 & 90,0 & 94,3 & 94,1 & 93,5 \\
\hline $\begin{array}{l}\text { andere Ver- } \\
\text { kehrsfläche }\end{array}$ & 2,3 & - & - & 1,4 & 2,2 & - & 1,2 & - & - & 0,2 & 0,4 & - \\
\hline Gesamt & 100,0 & 100,0 & 100,0 & 100,0 & 100,0 & 100,0 & 100,0 & 100,0 & 100,0 & 100,0 & 100,0 & 100,0 \\
\hline$N$ & 43 & 25 & 10 & 216 & 597 & 48 & 82 & 104 & 70 & 2.417 & 6.767 & 245 \\
\hline
\end{tabular}

Tabelle 34: Flächennutzung nach Fahrtrichtung und Altersgruppe in Straßen mit Radfahrstreifen (Anteil nach Altersgruppe in \%)

\begin{tabular}{|c|c|c|c|c|c|c|c|c|c|c|c|c|}
\hline \multicolumn{13}{|c|}{ Radfahrstreifen } \\
\hline & \multicolumn{12}{|c|}{ Anteil Fahrtrichtung und Alter [\%] } \\
\hline & links & \multicolumn{5}{|c|}{ regelwidrig links } & \multicolumn{6}{|c|}{ rechts } \\
\hline $\begin{array}{l}\text { genutzte } \\
\text { Fläche }\end{array}$ & $<10 \mathrm{~J}$ & 10-14 J. & $15-17 \mathrm{~J}$. & $18-24 \mathrm{~J}$. & $25-65 \mathrm{~J}$. & $>65 \mathrm{~J}$ & $<10 \mathrm{~J}$ & $10-14 \mathrm{~J}$. & $15-17 \mathrm{~J}$. & $18-24 \mathrm{~J}$. & $25-65 \mathrm{~J}$. & $>65 \mathrm{~J}$. \\
\hline $\begin{array}{l}\text { Radverkehrs- } \\
\text { anlage }\end{array}$ & 9,7 & 19,8 & 46,7 & 15,4 & 18,1 & 29,2 & 47,5 & 80,3 & 95,5 & 94,5 & 93,2 & 96,1 \\
\hline Gehweg & 88,7 & 80,2 & 53,3 & 82,4 & 80,5 & 68,8 & 51,8 & 19,1 & 4,1 & 2,6 & 3,5 & 2,8 \\
\hline Fahrbahn & - & - & - & 0,4 & 0,6 & 2,1 & - & 0,3 & 0,4 & 2,8 & 3,3 & 0,9 \\
\hline $\begin{array}{l}\text { andere } \\
\text { Verkehrsflä- } \\
\text { che }\end{array}$ & 1,6 & - & - & 1,8 & 0,8 & - & 0,7 & 0,3 & - & 0,1 & 0,1 & 0,2 \\
\hline Gesamt & 100,0 & 100,0 & 100,0 & 100,0 & 100,0 & 100,0 & 100,0 & 100,0 & 100,0 & 100,0 & 100,0 & 100,0 \\
\hline$N$ & 62 & 86 & 30 & 272 & 842 & 48 & 139 & 304 & 245 & 4.073 & 13.709 & 643 \\
\hline
\end{tabular}

Tabelle 35: Flächennutzung nach Fahrtrichtung und Altersgruppe in Straßen mit Schutzstreifen (Anteil nach Altersgruppe in \%)

\begin{tabular}{|c|c|c|c|c|c|c|c|c|c|c|c|c|}
\hline \multicolumn{13}{|c|}{ Schutzstreifen } \\
\hline & \multicolumn{12}{|c|}{ Anteil Fahrtrichtung und Alter [\%] } \\
\hline & links & \multicolumn{5}{|c|}{ regelwidrig links } & \multicolumn{6}{|c|}{ rechts } \\
\hline $\begin{array}{c}\text { genutzte Flä- } \\
\text { che }\end{array}$ & $<10 \mathrm{~J}$ & 10-14 J. & $15-17 \mathrm{~J}$. & 18-24 J. & $25-65 \mathrm{~J}$. & $>65 \mathrm{~J}$ & $<10 \mathrm{~J}$ & $10-14 \mathrm{~J}$. & $15-17 \mathrm{~J}$. & $18-24 \mathrm{~J}$. & $25-65 \mathrm{~J}$. & $>65 \mathrm{~J}$. \\
\hline $\begin{array}{l}\text { Radverkehrs- } \\
\text { anlage }\end{array}$ & 1,7 & 7,0 & - & 10,1 & 12,0 & 14,7 & 25,9 & 63,3 & 89,2 & 94,9 & 93,3 & 91,4 \\
\hline Gehweg & 96,7 & 93,0 & 100,0 & 89,2 & 87,6 & 85,3 & 73,2 & 34,0 & 7,9 & 4,2 & 5,7 & 7,7 \\
\hline Fahrbahn & - & - & - & 0,6 & 0,2 & - & 0,9 & 2,3 & 2,8 & 0,9 & 1,0 & 0,9 \\
\hline $\begin{array}{l}\text { andere Ver- } \\
\text { kehrsfläche }\end{array}$ & 1,7 & - & - & - & 0,2 & - & - & 0,5 & - & - & - & - \\
\hline Gesamt & 100,0 & 100,0 & 100,0 & 100,0 & 100,0 & 100,0 & 100,0 & 100,0 & 100,0 & 100,0 & 100,0 & 100,0 \\
\hline $\mathrm{N}$ & 60 & 71 & 13 & 158 & 523 & 34 & 112 & 215 & 316 & 2.896 & 8.387 & 549 \\
\hline
\end{tabular}




\section{Lichtsignalgeregelte Knotenpunkte}

An lichtsignalgeregelten Knotenpunkten fuhren Über-65-Jährige anteilig etwas häufiger regelwidrig links auf Radweg- bzw. auf Gehwegfurten (Bild 54). Eine grundsätzlich höhere Gefährdung aus diesem Verhalten konnte aus den Unfalldaten jedoch nicht ermittelt werden.

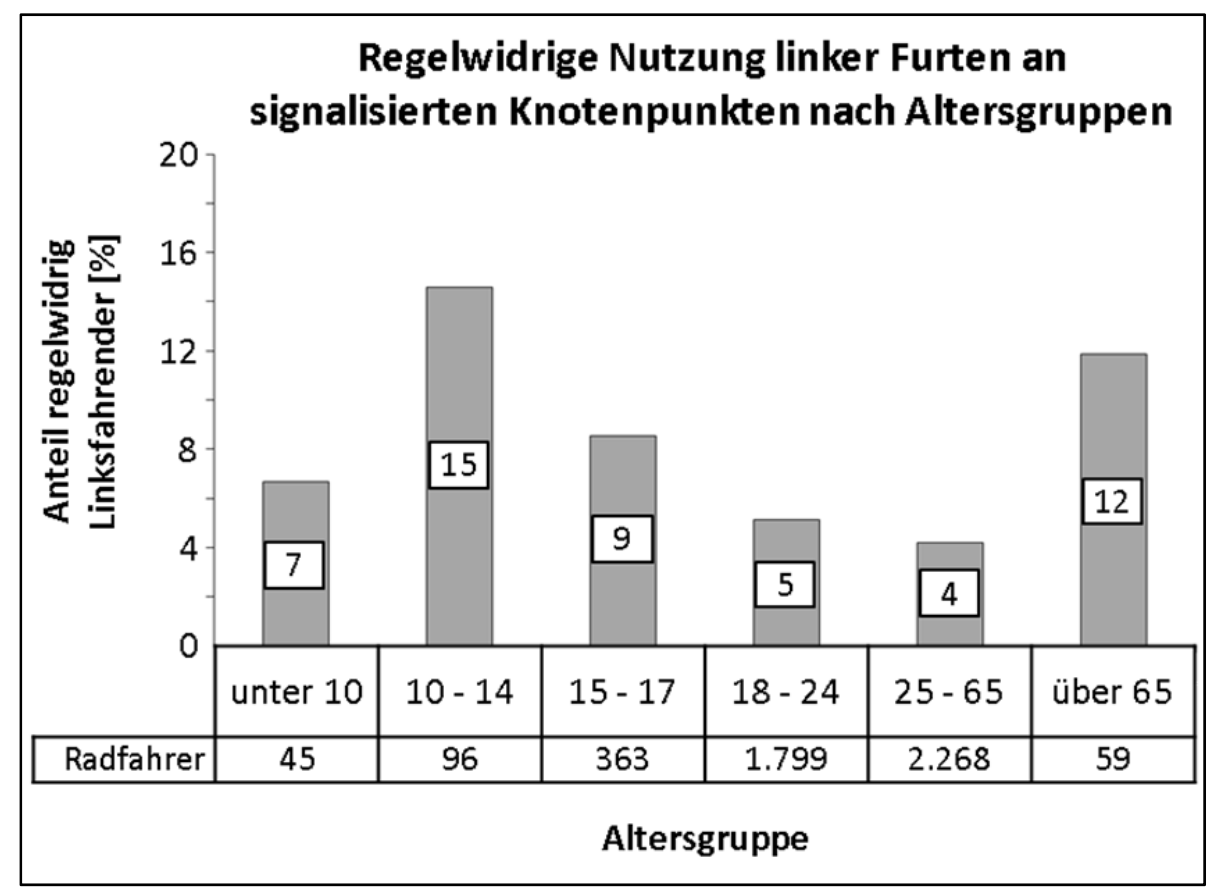

Bild 54: Regelwidrige Nutzung linker Furten nach Altersgruppe an lichtsignalgeregelten Knotenpunkten

\subsubsection{Zusammenfassung}

An Streckenabschnitten hatten Über-65-Jährige ein im Durchschnitt etwa doppelt so hohes Unfallrisiko (Unfallrate) wie 25- bis 65-Jährige. An lichtsignalgeregelten Knotenpunkten konnte kein Zusammenhang zwischen dem Alter der Radfahrer und der Anzahl der Unfälle festgestellt werden.

An Streckenabschnitten und Knotenpunkten traten bei Unfällen älterer Radfahrer anteilig häufiger schwere Unfälle auf. Ein ähnliches Bild zeigte sich an lichtsignalgeregelten Knotenpunkten.

Wurden an Streckenabschnitten die von Radfahrern verursachten Unfälle betrachtet, dann dominierten Einbiegen-/ Kreuzen- sowie Fahrunfälle. Für die Über-65-Jährigen lag der Anteil der Fahrunfälle gegenüber der Altersklasse der Unter-65-Jährigen jedoch auf einem höheren Niveau (30 \% zu 22 \%).

An lichtsignalgeregelten Knotenpunkten dominierten Einbiegen-/ Kreuzen- und Abbiege-Unfälle unabhängig von der Altersklasse. Allerdings nahmen die Einbiegen-/ Kreuzen-Unfälle bei Über-65Jährigen dabei einen größeren Anteil an den beiden Unfalltypen ein.

Auf Streckenabschnitten fuhren Über-65-Jährige geringfügig häufiger in regelwidrig linker Fahrtrichtung als 25- bis 65-Jährige. Dies spiegelte sich jedoch nicht in höheren Unfallzahlen wieder. An lichtsignalgeregelten Knotenpunkten fuhren Über-65-Jährige häufiger regelwidrig links auf Radweg- bzw. Gehwegfurten als 25- bis 65-Jährige. Eine höhere Gefährdung für Über-65-Jährige konnte für beide regelwidrige Verhaltensweisen jedoch nicht ermittelt werden. 


\subsection{Radfahrergeschwindigkeiten, Unfallgeschehen und Regelbefolgung}

Es wird vermutet, dass die Geschwindigkeiten von Radfahrern Einfluss auf das zukünftige Unfallgeschehen und Verkehrsverhalten haben. Im Folgenden wurden daher Erkenntnisse aus den einzelnen Untersuchungsschritten zur Wirkung der Geschwindigkeiten des Radverkehrs auf die Unfallanzahl, die Unfallschwere, die Unfalltypenverteilung und die Regelbefolgung zusammengefasst. Dabei wurde wiederum jeweils nach Streckenabschnitten und lichtsignalgeregelten Knotenpunkten differenziert.

\subsubsection{Radfahrergeschwindigkeiten und Unfallanzahl}

\section{Streckenabschnitte}

Die makroskopische Unfallauswertung zeigte einen leichten Anstieg der jeweiligen Unfallanzahl bei steigenden Werten der $\mathrm{v}_{85}$-Radgeschwindigkeiten (Bild 55).

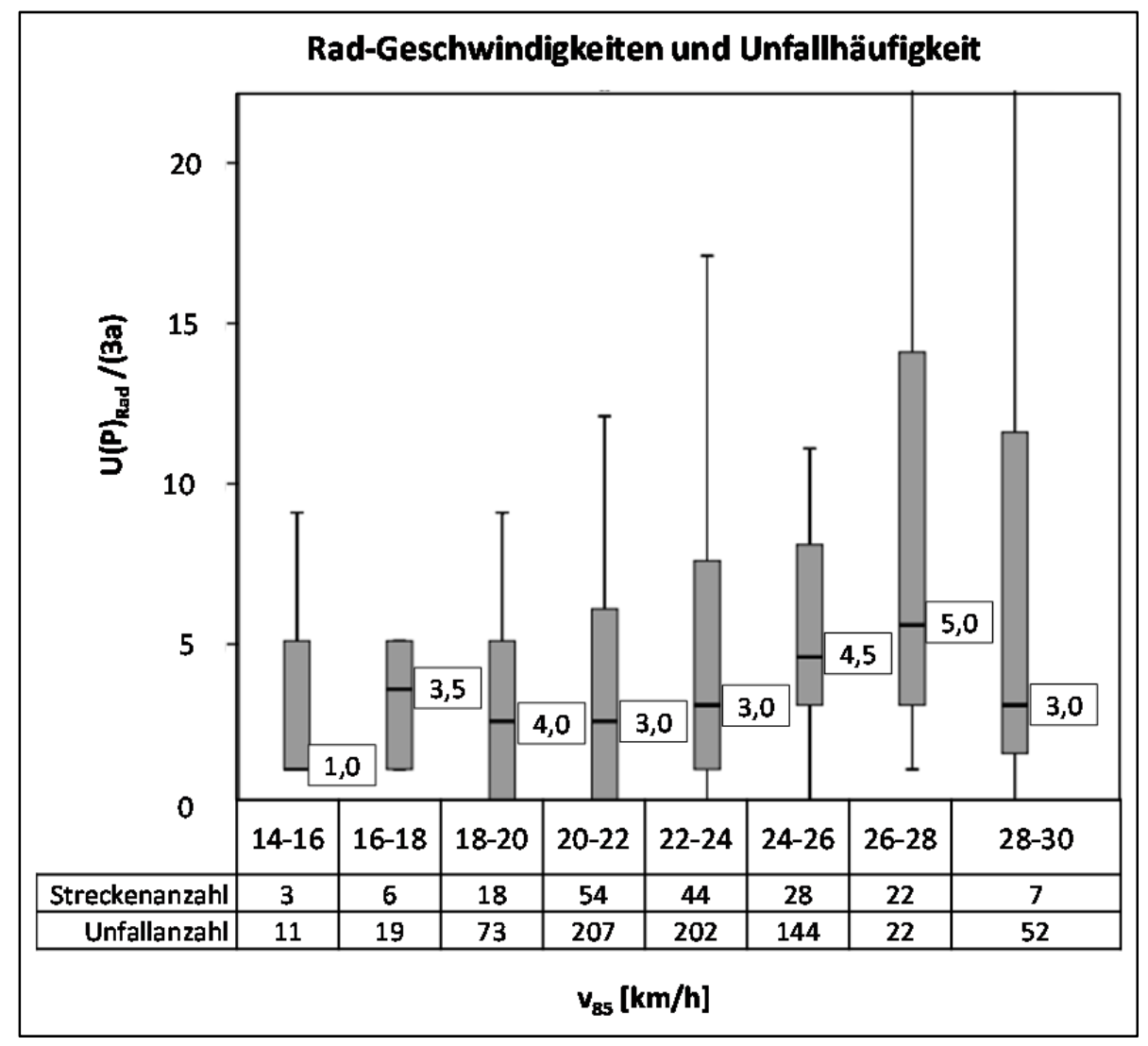

Bild 55: Radgeschwindigkeiten in $\mathrm{v}_{85}$ und Unfallanzahl 
In einem separaten Unfallmodell wurden die Unfälle der 200 Streckenabschnitte auf mögliche Einflüsse durch Unterschiede in den gefahrenen Geschwindigkeiten des Radverkehrs untersucht. Dazu wurden die auf den Streckenabschnitten gemessenen Geschwindigkeiten herangezogen. Als beste beschreibende geschwindigkeitsspezifische Größe zeigte sich dabei die $\mathrm{v}_{85}{ }^{36}$. Allerdings konnten nur $5,3 \%$ der Streuung in den Unfallzahlen über die $v_{85}$ erklärt werden (siehe Tabelle 36, letzte Spalte). Andere Einflussgrößen wie die Radverkehrsstärke und Streckenlänge erklärten gemeinsam im Hauptunfallmodell hingegen etwa $33 \%$ der Streuung der Unfallanzahl (siehe Kapitel 4.2.1).

Tabelle 36: separates Unfallmodel zur Ermittlung des Einflusses der Geschwindigkeiten im Radverkehr

\begin{tabular}{|l|c|c|c|c|c|}
\hline \multicolumn{1}{|c|}{ Modell } & Parameter & Koeffizienten & Signifikanz & Anpassungsgüte & Streuung [\%] \\
\hline Nullmodell & & & & 6,026 & 100 \\
\hline Teilmodell $\mathbf{v}_{85}$ & Konstante & $-2,522$ & 0,048 & \multirow{2}{*}{5,761} & 94,7 \\
\hline & $\mathrm{V}_{85}$ & 0,05 & 0,000 & & \\
\hline Formel 3: & & $U(P)=e^{-2,522} * e^{0,05 * v} 85$ \\
\end{tabular}

Auch wenn ein statistischer Zusammenhang zwischen Unfallhäufigkeit und Radgeschwindigkeiten im separaten Unfallmodell und der makroskopischen Analyse festgestellt werden konnte, konnte der Einfluss der Geschwindigkeit nicht getrennt von der Radverkehrsstärke betrachtet werden. Sowohl bei zunehmenden Radverkehrsstärken, als auch bei zunehmenden Rad-Geschwindigkeiten zeigte sich ein Anstieg der Unfallzahlen. Die makroskopische Analyse des Zusammenhangs zwischen Radverkehrsstärke und Radgeschwindigkeit zeigte jedoch auch, dass mit steigenden Radverkehrsstärken die Radgeschwindigkeiten zunahmen (Bild 56). Da die Auswertung der Geschwindigkeiten frei fahrende Radfahrer betrachtete, ist anzunehmen, dass trotz höherer Radverkehrsstärken und stärkerer Pulkung der Radfahrer die Anzahl schnell und freifahrender Radfahrer ansteigt. 


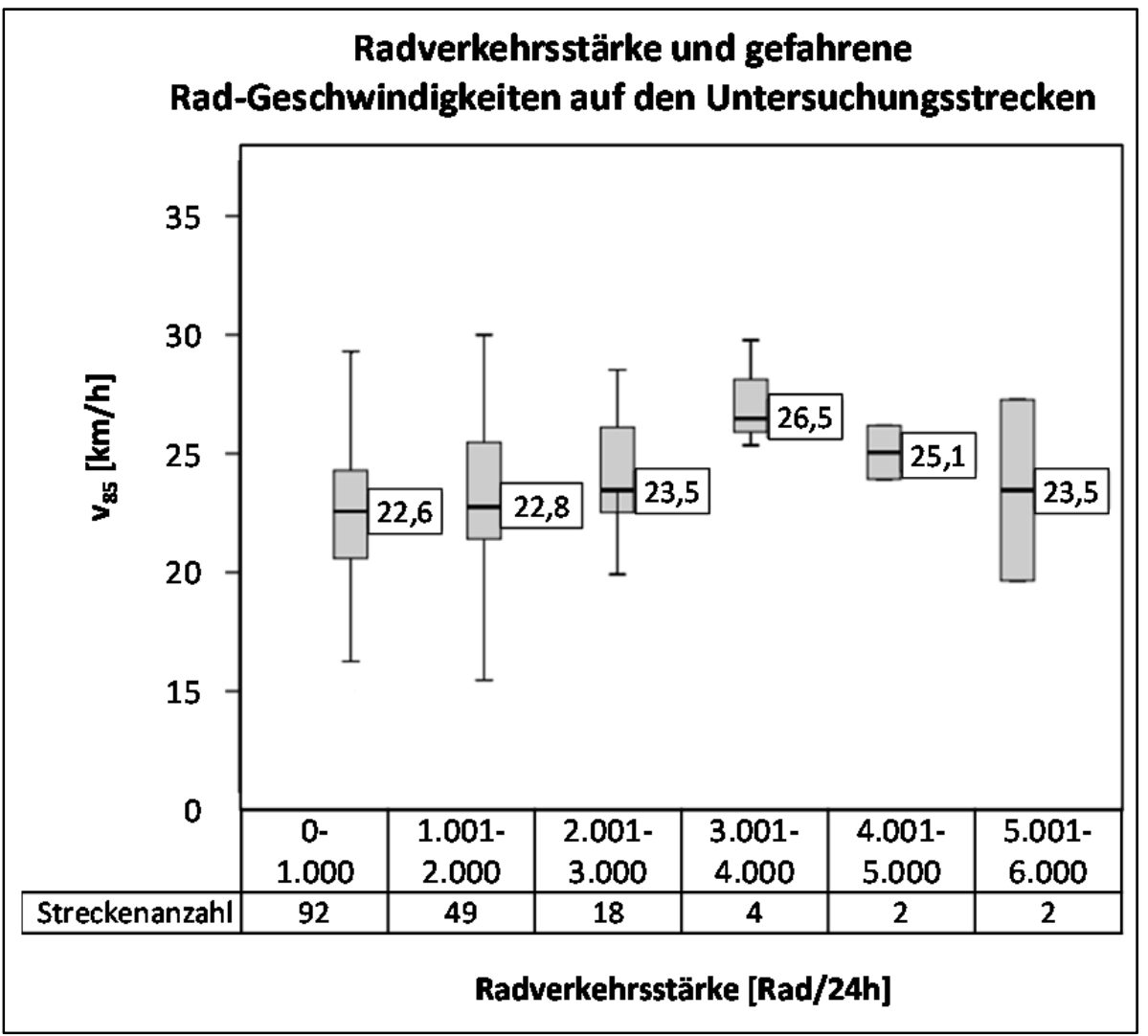

Bild 56: Radverkehrsstärke in Radverkehrsstärkeklassen und Radgeschwindigkeiten in $\mathrm{v}_{85}{ }^{37}$

In das Hauptmodell (siehe Kapitel 4.2.1) wurde die Rad-Geschwindigkeit als Einflussgröße aus modelltheoretischen Gründen nicht aufgenommen, da die Radverkehrsstärke einen größeren Teil an systematischer Streuung erklären konnte. zwischen dem 25- und $75 \%$ Quartilen). 


\section{Lichtsignalgeregelte Knotenpunkte}

Bei den Videobeobachtungen an den lichtsignalgeregelten Knotenpunkten wurden keine Geschwindigkeiten gemessen. Hinweise auf einen Einfluss der Geschwindigkeiten ergaben sich jedoch aus der makroskopischen Unfallanalyse: An den Knotenpunkten, an denen in den polizeilichen Unfalldaten die Ursache 12 und 13 (nicht angepasste Geschwindigkeit der Radfahrer) genannt wurden, lag auch die durchschnittliche Unfallanzahl höher. Zu berücksichtigen ist allerdings, dass das Kollektiv mit 18 Nennungen sehr klein war und dass an Knotenpunkten mit vielen Unfällen auch diese Ursachen häufiger auftreten konnten.

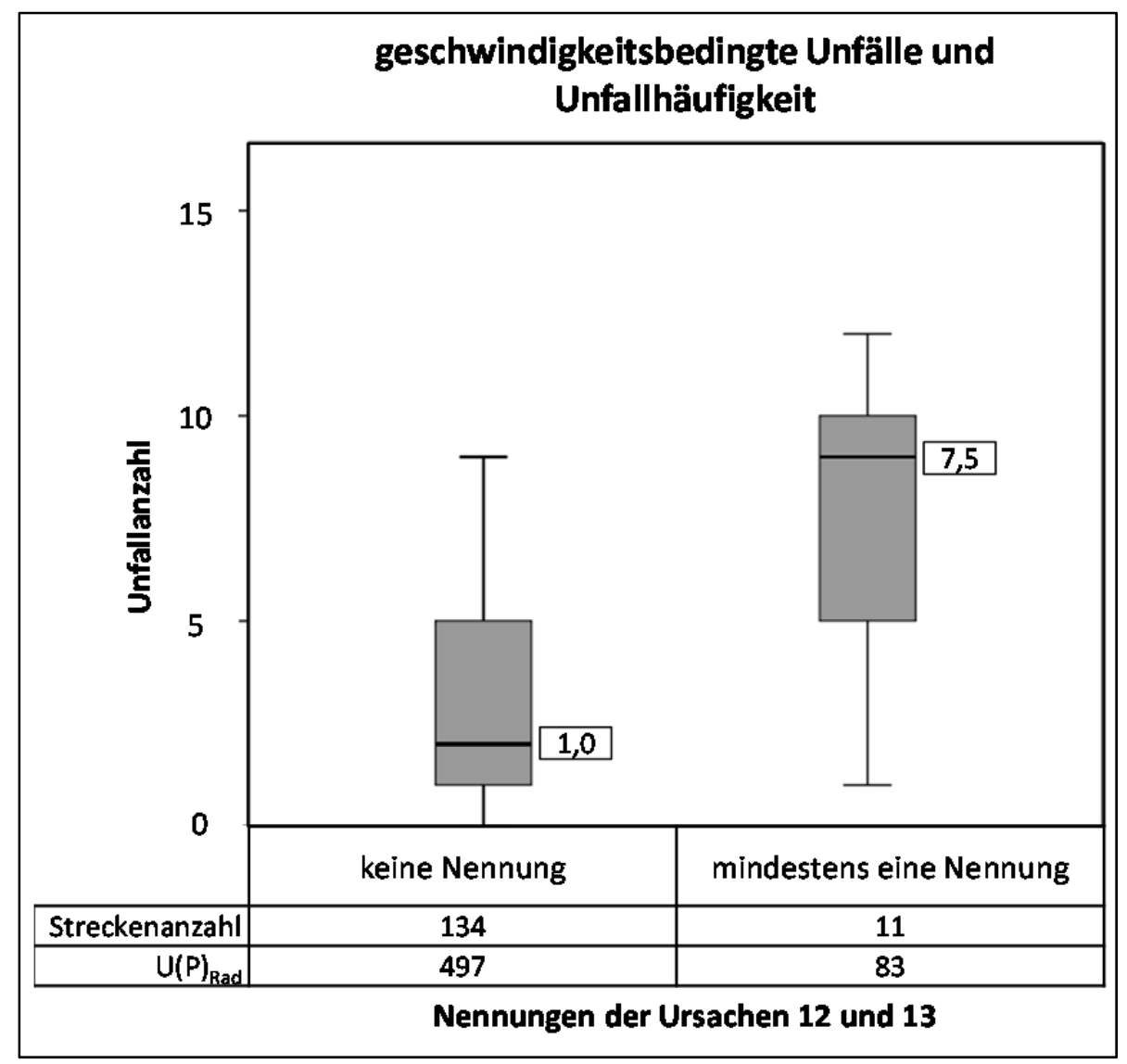

Bild 57: Nennung der Unfallursache 12 und 13 „nicht angepasste Geschwindigkeit“ und Unfallanzahl 


\subsubsection{Radfahrergeschwindigkeiten und Unfallschwere}

\section{Streckenabschnitte}

Zur Beurteilung des Einflusses der Geschwindigkeit auf die Unfallschwere wurden in der Makroanalyse die Untersuchungsstrecken in zwei Klassen aufgeteilt. Dabei wurde zwischen den Strecken mit geringeren als im Mittel auftretenden $v_{85}$ (ca. $23 \mathrm{~km} / \mathrm{h}$ ) und solchen mit höherer $\mathrm{v}_{85}$ als dieser Mittelwert unterschieden. Entsprechend dieser Aufteilung der Erhebungsstrecken wurde das Unfallgeschehen mit Personenschaden und Radbeteiligung nach den Unfallkategorien ausgewertet (Bild 58).

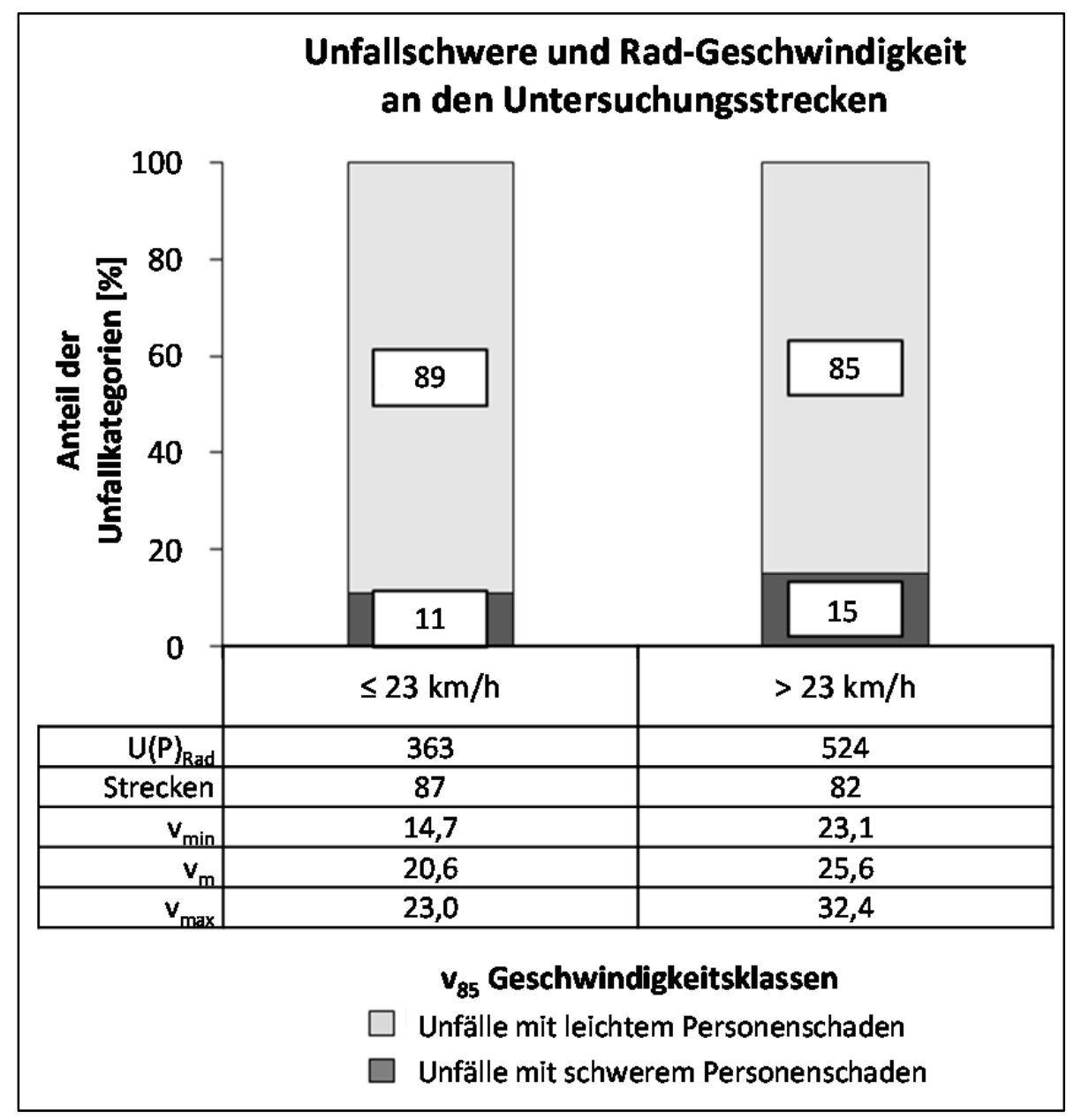

Bild 58: Unfallstruktur und $\mathrm{v}_{85}$ der Radfahrer in zwei Klassen 
In der Gegenüberstellung beider Klassen zeigte sich eine Verschiebung der Anteile der schweren Unfälle. Für die Geschwindigkeitsklasse „, $\mathrm{V}_{85} \leq 23 \mathrm{~km} / \mathrm{h}$ “ lag der Anteil schwerer Unfälle bei $11 \%$, während er auf den Strecken der Geschwindigkeitsklasse „, $\mathrm{V}_{85}>23 \mathrm{~km} / \mathrm{h}$ “ auf $15 \%$ anstieg. Um den Einfluss der Geschwindigkeit auf die Unfallschwere besser zu quantifizieren, wurden die Kennwerte der $\mathrm{v}_{85}$ auf den 200 Streckenabschnitten klassifiziert. Für jede Klasse wurde die mittlere Unfallschwere (Anteil Radverkehrsunfälle mit schwerem Personenschaden an Radverkehrsunfällen mit Personenschaden) ermittelt (Bild 59). Im Ergebnis zeigten sich höhere Anteile schwerer Unfälle für Streckenabschnitte mit höheren $\mathrm{v}_{85}$.

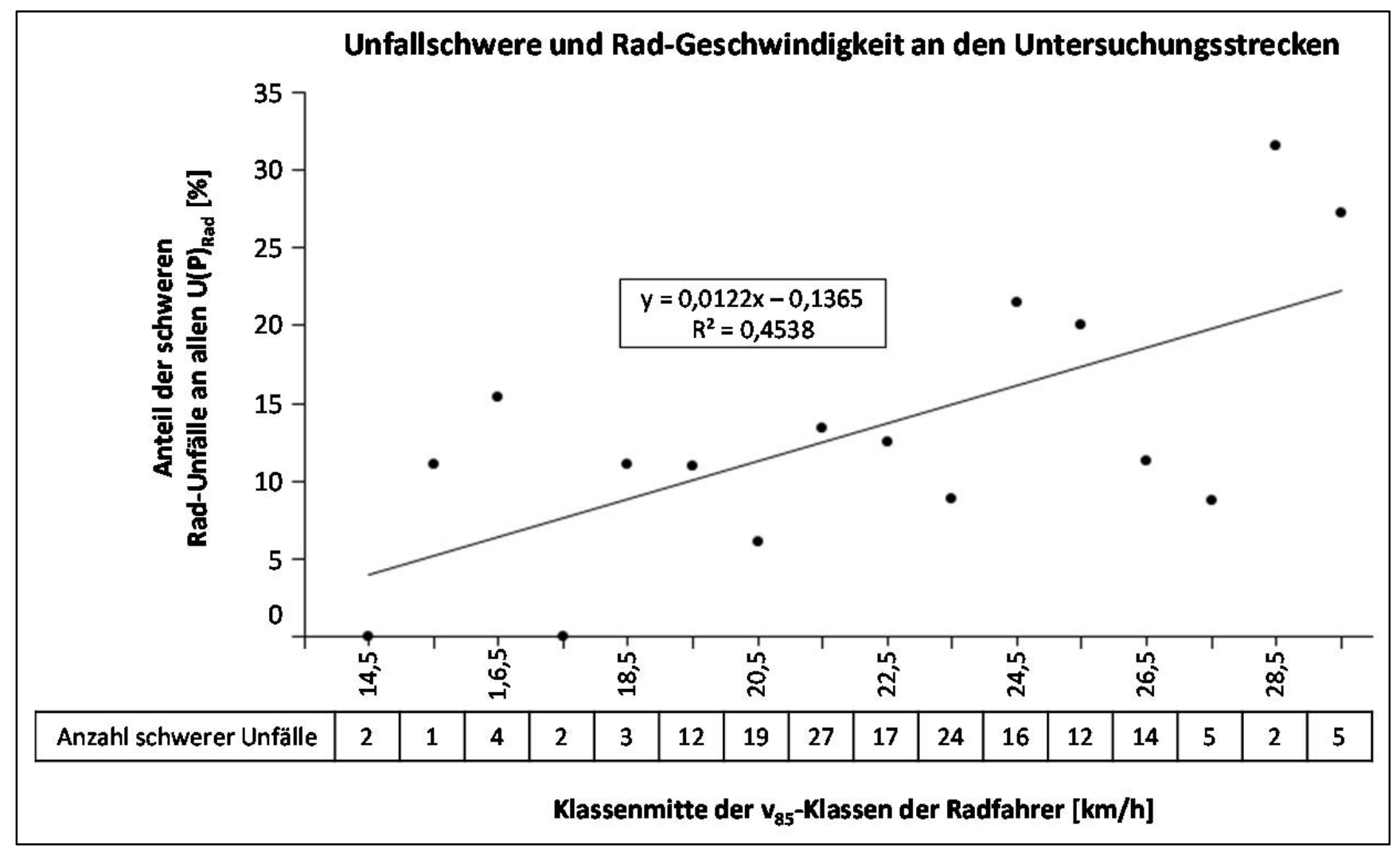

Bild 59: Radfahrergeschwindigkeit (gefahrene $v_{85}$ (klassifiziert)) ${ }^{38}$ und Unfallschwere (Anteil schwerer Radverkehrsunfälle (schwerer Personenschaden) an allen Radverkehrsunfällen mit Personenschaden)

\section{Lichtsignalgeregelte Knotenpunkte}

Die in Kapitel 4.4.1 genannten unfallauffälligen Knotenpunkte gaben keine Hinweise auf einen Einfluss der Radfahrergeschwindigkeiten auf die Unfallschwere. Alle Unfälle hatten leichte Verletzungen zur Folge. 


\subsubsection{Radfahrergeschwindigkeiten und Unfalltypen}

\section{Streckenabschnitte}

Zur Beurteilung des Einflusses der Geschwindigkeit auf die Unfalltypen wurden die Untersuchungsstrecken in der Makroanalyse in zwei Klassen aufgeteilt. Dabei wurde zwischen den Strecken mit geringeren als im Mittel auftretenden $v_{85}$ (ca. $23 \mathrm{~km} / \mathrm{h}$ ) und solchen mit höherer $\mathrm{v}_{85}$ als dieser Mittelwert unterschieden. Entsprechend dieser Aufteilung der Erhebungsstrecken wurde das Unfallgeschehen mit Personenschaden und Radbeteiligung auf den Untersuchungsstrecken nach den Unfalltypen ausgewertet. Um Verschiebungen in den Anteilen der relevanten Unfalltypen zu verdeutlichen, wurden Unfälle des Typs 7 „Sonstige“ nicht mit betrachtet. ${ }^{39}$

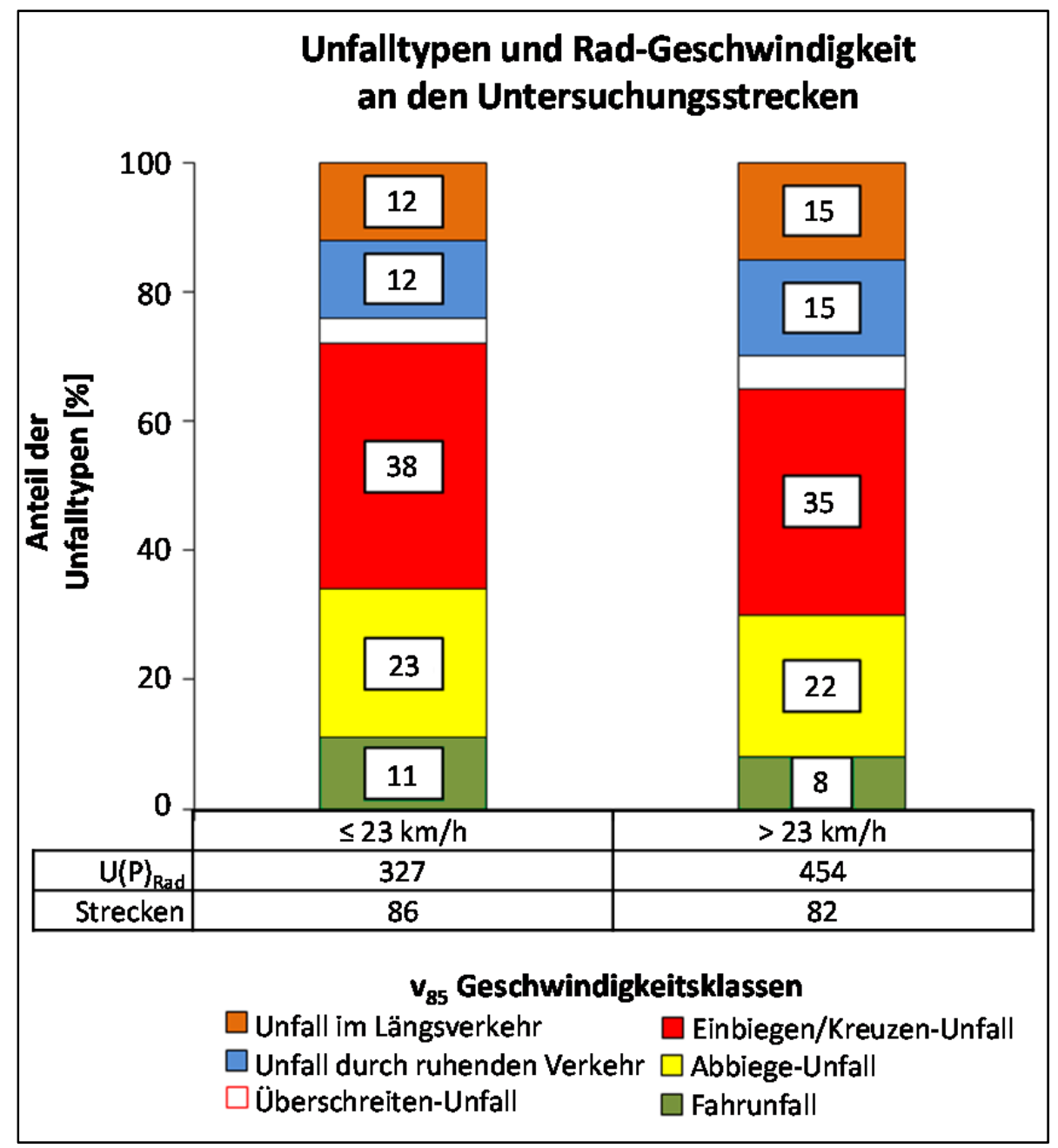

Bild 60: Unfalltypen (Anteile, ohne Typ 7) und $\mathbf{v}_{85}$ der Radfahrer in zwei Klassen 
Die Gegenüberstellung beider Klassen zeigte eine geringe Verschiebung der Anteile der Unfalltypen „Unfall im Längsverkehr“ und „Unfall durch ruhenden Verkehr“. Gegenüber der Geschwindigkeitsklasse „, $\mathrm{V}_{85} \leq 23 \mathrm{~km} / \mathrm{h}^{\prime}$ (12\%) lag der Anteil der Unfälle im Längsverkehr für die Geschwindigkeitsklasse „V $\mathrm{V}_{85}>23 \mathrm{~km} / \mathrm{h}$ “ auf einem höheren Niveau (15\%) In gleichem Maß veränderten sich die Unfälle durch ruhenden Verkehr. Die Fahr- und Einbiegen-/ Kreuzen-Unfälle wiesen entsprechend auf Strecken der Geschwindigkeitsklasse „, $\mathrm{V}_{85}>23 \mathrm{~km} / \mathrm{h}$ “ etwas geringere Anteile auf als auf Strecken der Geschwindigkeitsklasse „, $\mathrm{V}_{85} \leq 23 \mathrm{~km} / \mathrm{h}$ “ (Bild 60).

Bei einer Aufschlüsselung nach Anlagentypen und Unfallabläufen ergaben sich in der Mikroanalyse Hinweise, dass sich auf Streckenabschnitten mit „, $\mathrm{V}_{85}>23 \mathrm{~km} / \mathrm{h}$ " gerade bei Fahrbahn- und Radfahrstreifenführungen anteilig mehr Unfälle beim Ein- bzw. Aussteigen von Personen aus parkenden Pkw ereigneten (Unfalltyp 581, Tabelle 37).

Bei Mischverkehr auf der Fahrbahn und bei Radwegführung ereigneten sich auf Streckenabschnitten mit „, $\mathrm{V}_{85}>23 \mathrm{~km} / \mathrm{h}$ “ vermehrt Unfälle im Längsverkehr (Unfalltypen 651 und 681, Tabelle 37):

- im Mischverkehr auf der Fahrbahn waren Pkw die häufigsten Unfallgegner beim Nebeneinanderfahren (Typ 651). Bei einem Unfall fuhr ein Radfahrer rechts an einem Pkw vorbei, der zum Einparken angehalten hatte, bei den anderen Unfällen wurden Radfahrer von überholenden Pkw berührt. Die Radfahrer waren zwischen 32 bzw. 43 Jahre alt. Ein Einfluss höherer Fahrgeschwindigkeiten konnte nicht ausgeschlossen werden, war aber in den Unfallbeschreibungen nicht ausdrücklich vermerkt. Auffällig war, dass sich diese Überholunfälle in einer Straße mit zwei Richtungsfahrstreifen ereigneten.

- auf dem Radweg ließ das Alter der beteiligten Radfahrer (zumeist zwischen 28 bis 42 Jahren, bei einem Unfall 57 Jahre) auch bei den Unfällen unter begegnenden Radfahrern (Typ 681) eine möglicherweise höhere Geschwindigkeit vermuten. Auffällig war, dass sich fast alle dieser Unfälle auf Radwegen mit einer geringeren Breite als 1,6 m ereigneten.

Speziell auf Radwegen zeigte sich auf Streckenabschnitten mit „, $\mathrm{V}_{85}>23 \mathrm{~km} / \mathrm{h}$ “ ein deutlich erhöhter Anteil von Einbiegen-/ Kreuzen-Unfällen bei denen der Radfahrer die Furt von rechts kommend befuhr (Typ 342). Ein Zusammenhang zur gefahrenen Geschwindigkeit konnte jedoch nicht hergestellt werden, da die gemessenen Geschwindigkeiten nur von in rechter Richtung gefahrenen Radfahrern gemessen wurde. 
Tabelle 37: Unfalltypen nach Anlagentyp und Radfahrergeschwindigkeit in zwei Klassen

\begin{tabular}{|c|c|c|c|c|c|c|}
\hline \multicolumn{7}{|c|}{ Anteil der Unfalltypen [\%] } \\
\hline \multirow{2}{*}{$\begin{array}{l}\text { Dreistelliger } \\
\text { Unfalltyp nach } \\
\text { M Uko }\end{array}$} & \multicolumn{2}{|c|}{ Mischverkehr } & \multicolumn{2}{|c|}{ Radweg } & \multicolumn{2}{|c|}{ Radfahrstreifen } \\
\hline & $v_{85}<23 \mathrm{~km} / \mathrm{h}$ & $v_{85}>23 \mathrm{~km} / \mathrm{h}$ & $v_{85}<23 \mathrm{~km} / \mathrm{h}$ & $v_{85}>23 \mathrm{~km} / \mathrm{h}$ & $v_{85}<23 \mathrm{~km} / \mathrm{h}$ & $v_{85}>23 \mathrm{~km} / \mathrm{h}$ \\
\hline 203 & & & 2 & & & \\
\hline 204 & 11 & & & & & \\
\hline 211 & 11 & & & & & 5 \\
\hline 223 & & & 2 & 2 & & \\
\hline 224 & & & & 6 & & \\
\hline 232 & 11 & & & & 20 & 5 \\
\hline 243 & & & 17 & 9 & & \\
\hline 244 & & & & 2 & & \\
\hline 299 & & & & 2 & & 5 \\
\hline 302 & & 6 & & & 10 & \\
\hline \multicolumn{7}{|l|}{321} \\
\hline 341 & & & 11 & 13 & & \\
\hline 342 & 11 & 6 & 7 & 25 & & \\
\hline 371 & & 6 & 4 & & 10 & 10 \\
\hline 372 & & & 9 & 8 & & \\
\hline 399 & 11 & & 2 & & & \\
\hline 401 & & & 4 & 2 & & \\
\hline 421 & & & 7 & 2 & 10 & \\
\hline \multicolumn{7}{|l|}{499} \\
\hline 501 & & 6 & & & & \\
\hline 542 & & & & & 10 & \\
\hline 551 & & & & & & 5 \\
\hline 581 & & 13 & & & & 24 \\
\hline 631 & & & 2 & & & \\
\hline 651 & & 19 & 11 & & & 5 \\
\hline 681 & & & 13 & 19 & & \\
\hline 699 & & 13 & 4 & & & \\
\hline 799 & 11 & 6 & & 4 & 20 & 10 \\
\hline Summe & 100 & 100 & 100 & 100 & 100 & 100 \\
\hline$N$ U(P) & 9 & 16 & 46 & 53 & 10 & 21 \\
\hline
\end{tabular}




\section{Lichtsignalgeregelte Knotenpunkte}

Aus Daten der Makroanalyse ergab sich, dass bei Unfällen mit nicht angepassten Geschwindigkeiten der Radfahrer (Unfallursachen 12 und 13) deutlich höhere Anteile von Unfällen mit schwerem Personenschaden als bei Unfällen ohne diese Ursache auftraten. Zu berücksichtigen war allerdings ein sehr kleines Kollektiv dieser geschwindigkeitsbeeinflussten Unfälle (Tabelle 61).

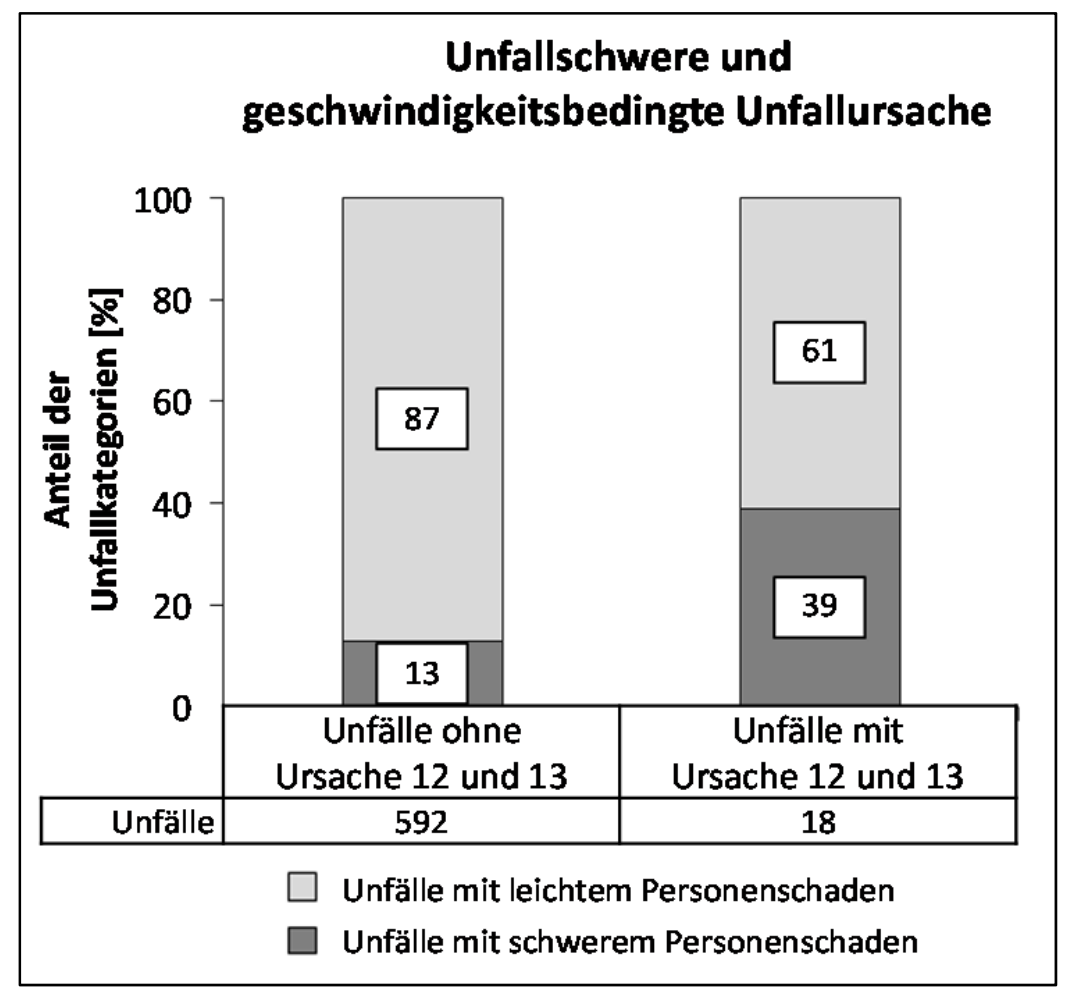

Bild 61: Nennung der Unfallursache 12 und 13 „nicht angepasste Geschwindigkeit“ und Unfallschwere

\subsubsection{Radfahrergeschwindigkeiten und Regelbefolgung}

Weder für die Streckenabschnitte noch für die lichtsignalgeregelten Knotenpunkte konnten Zusammenhänge zwischen höheren Geschwindigkeiten und Regelverstößen ermittelt werden.

\subsubsection{Zusammenfassung}

Ein Teilmodell des Unfallmodells zeigte bei höheren Geschwindigkeiten von Radfahrern auf Streckenabschnitten eine leicht erhöhte Unfallanzahl. Dieser Einfluss war jedoch durch andere Einflussgrößen, wie insbesondere der Radverkehrsstärke, überlagert. Unfallauffällige lichtsignalgeregelte Knotenpunkte gaben Hinweise, dass höhere Geschwindigkeiten von Radfahrern auf eine höhere Anzahl von Unfällen hinwirkten.

An Streckenabschnitten ereigneten sich bei höherer $\mathrm{v}_{85}$ des Radverkehrs anteilig mehr schwere Unfälle. An lichtsignalgeregelten Knotenpunkten ergaben sich keine Hinweise auf Einflüsse der Radfahrergeschwindigkeiten auf die Unfallschwere.

In Straßen mit höheren Radfahrergeschwindigkeiten ereigneten sich vermehrt Unfälle im Längsverkehr und Unfälle durch ruhenden Verkehr. Unfallauffällige lichtsignalisierte Knotenpunkte deuteten darauf hin, dass bei höheren Radfahrergeschwindigkeiten und Radwegführung vermehrt Unfälle zwischen rechtsabbiegenden Kfz und Radfahrern in rechter Richtung auftreten können. 


\subsection{Zusammenfassung der Erkenntnisse}

Für den heutigen Radverkehr zeigten sich für die untersuchten Einflussbereiche folgende Einflüsse:

\section{Radverkehrsstärken}

An Streckenabschnitten mit Anschlussknoten bedingten höhere Radverkehrsstärken eine höhere Unfallanzahl. An lichtsignalgeregelten Knotenpunkten konnten schon bei niedrigen Radverkehrsstärken hohe Unfallbelastungen auftreten. Ein Zusammenhang zwischen höheren Radverkehrsstärken und höheren Unfallbelastungen konnte für lichtsignalgeregelte Knotenpunkte nicht ermittelt werden.

An Streckenabschnitten zeigten sich mit höheren Radverkehrsstärken höhere Anteile von Unfällen mit schwerem Personenschaden. An lichtsignalgeregelten Knotenpunkten konnten schon bei niedrigen Radverkehrsstärken hohe Anteile schwerer Unfälle auftreten. Ein verallgemeinerbarer Zusammenhang zwischen Radverkehrsstärken und Unfallschwere konnte an lichtsignalgeregelten Knotenpunkten nicht ermittelt werden.

Bei höheren Radverkehrsstärken verschoben sich die Unfalltypen an Streckenabschnitten von Abbiegen- Und Einbiegen-/ Kreuzen-Unfällen zu Unfälle im Längsverkehr und Unfälle durch ruhenden Verkehr. Bei Radwegführung ereigneten sich bei höheren Radverkehrsstärken mehr Unfälle unter begegnenden Radfahrern auf dem Radweg. Die Unfälle dieses Typs ereigneten sich dabei auf Radwegen mit weniger als 1,6 $\mathrm{m}$ Breite.

An lichtsignalgeregelten Knotenpunkten zeigten sich unabhängig von den Radverkehrsstärken bei unterschiedlichen Führungsformen typische Unfälle. So ereigneten sich etwa bei Radwegführungen anteilig mehr Unfälle zwischen geradeausfahrenden Radfahrern und rechts abbiegenden $\mathrm{Kfz}$, bei Fahrbahnführung in der Tendenz mehr Unfälle mit überholenden Kfz. Es wurden keine belastbaren Zusammenhänge zwischen den Radverkehrsstärken und der Verteilung der Unfalltypen ermittelt.

Sicherheitsprobleme durch das regelwidrige Linksfahren bestanden auf Streckenabschnitten an den Anschlussknotenpunkten und vor allem bei Führung des Radverkehrs auf Radwegen. Die Anteile regelwidrig linksfahrender Radfahrer sanken bei höheren Radverkehrsstärken. Bereits bei geringen Stärken des regelwidrigen linken Radverkehrs traten jedoch bereits auffällig hohe Unfalldichten regelwidrig links Fahrender auf.

Ein Vergleich zwischen den Anteilen regelwidrig auf dem Gehweg Fahrender in den deutschen Untersuchungsstädten (8-10 \% der Radfahrer in rechter Fahrtrichtung in Straßen mit Radwegen) und im niederländischen Groningen (1\%) ließ vermuten, dass eine Höhentrennung zwischen Geh- und Radwegen die Gehwegnutzung erheblich reduzieren kann.

\section{Alter}

An Streckenabschnitten hatten Über-65-Jährige ein im Durchschnitt etwa doppelt so hohes Unfallrisiko (Unfallrate) wie 25- bis 65-Jährige. An lichtsignalgeregelten Knotenpunkten konnte kein Zusammenhang zwischen dem Alter der Radfahrer und der Anzahl der Unfälle festgestellt werden.

An Streckenabschnitten mit Anschlussknotenpunkten traten bei Unfällen älterer Radfahrer anteilig häufiger schwere Unfälle auf (25\% gegenüber $13 \%$ ). Ein ähnliches Bild zeigte sich an lichtsignalge- 
regelten Knotenpunkten: Im Gesamtunfallgeschehen der Radfahrer (erweitertes Unfallkollektiv ${ }^{40}$ ) lag der Anteil der Unfälle mit schwerem Personenschaden an lichtsignalgeregelten Knotenpunkten in der Altersklasse der Unter-65-Jährigen bei $14 \%$; in der Klasse der Über-65-Jährigen bei $27 \%$.

Wurden an Streckenabschnitten die von Radfahrern verursachten Unfälle betrachtet, dann dominierten Einbiegen-/ Kreuzen- sowie Fahrunfälle. Für die Über-65-Jährigen lag der Anteil der Fahrunfälle gegenüber den Unter-65-Jährigen jedoch auf einem höheren Niveau (30 \% zu 22 \%).

Auf Streckenabschnitten fuhren Über-65-Jährige geringfügig häufiger in regelwidrig linker Fahrtrichtung als 25- bis 65-Jährige. Dieses spiegelte sich jedoch nicht in höheren Unfallzahlen wieder. In Straßen mit Schutzstreifen fuhren Über-65-Jährige in rechter Richtung häufiger auf dem Gehweg als 25- bis 65-Jährige (8 \% gegenüber $6 \%$ ). Nach den polizeilichen Daten bestand hier aber - ebenso wie auch bei anderen Anlagentypen - keine besondere Unfallgefährdung.

An lichtsignalgeregelten Knotenpunkten dominierten Einbiegen-/ Kreuzen- und Abbiege-Unfälle unabhängig von der Altersklasse. Allerdings nahmen die Einbiegen-/ Kreuzen-Unfälle bei Über-65Jährigen dabei einen höheren Anteil an den beiden Unfalltypen ein. An lichtsignalgeregelten Knotenpunkten fuhren Über-65-Jährige häufiger regelwidrig links auf Radweg- bzw. Gehwegfurten als 25bis 65-Jährige. Eine höhere Gefährdung für Über-65-Jährige konnte für beide regelwidrige Verhaltensweisen jedoch nicht ermittelt werden.

\section{Radfahrergeschwindigkeiten}

Ein Teilmodell des Unfallmodells zeigte bei höheren Geschwindigkeiten von Radfahrern auf Streckenabschnitten eine leicht erhöhte Unfallanzahl. Dieser Einfluss war jedoch durch andere Einflussgrößen, wie insbesondere die Radverkehrsstärke, überlagert.

An Streckenabschnitten ereigneten sich bei höherer $\mathrm{v}_{85}$ des Radverkehrs anteilig mehr schwere Unfälle. Aus Nennungen der Unfallursache „nicht angepasste Geschwindigkeit“ bei einer kleinen Anzahl von Unfällen ergaben sich Hinweise, dass höhere Geschwindigkeiten von Radfahrern auf eine höhere Anzahl und schwerer verlaufender Unfälle hinwirken.

In Straßen mit höheren Radfahrergeschwindigkeiten ereigneten sich vermehrt Unfälle im Längsverkehr und Unfälle durch ruhenden Verkehr. Unfallauffällige signalisierte Knotenpunkte deuteten darauf hin, dass bei höheren Radfahrergeschwindigkeiten und Radwegführung vermehrt Unfälle zwischen rechtsabbiegenden Kfz und Radfahrern in rechter Richtung auftreten können. 


\section{Überblick der Einflüsse}

Die Tabelle 38 fasst die Einflüsse aus Radverkehrsstärke, demografischer Wandel und Radfahrergeschwindigkeit auf die Anzahl, Schwere und Typen der Radverkehrsunfälle zusammen. Als "stark" wurde ein Einfluss bezeichnet, wenn auf Grundlage eines großen Kollektivs deutliche Unterschiede zwischen den Ausprägungen der Einflussfaktoren - also z. B. zwischen verschiedenen Altersgruppen herausgearbeitet werden konnten. Als "leicht“ wurde ein Einfluss definiert, der nur auf Basis eines kleinen Kollektivs ermittelt werden konnte oder bei dem sich vergleichsweise geringe Unterschiede wie etwa Anteilsdifferenzen von bis zu 3 Prozentpunkten - zeigten. Ermittelte Einflüsse, die aber durch andere Einflussfaktoren überlagert waren, wurden weder als "stark" noch als "leicht" eingestuft.

Tabelle 38: Übersicht der ermittelten Einflüsse aus Radverkehrsstärke, demografischer Wandel und Radfahrergeschwindigkeit auf die Anzahl, Schwere und Typen der Radverkehrsunfälle

\begin{tabular}{|c|c|c|c|c|c|c|}
\hline \multirow[b]{2}{*}{ Einflussfaktor } & \multicolumn{3}{|c|}{$\begin{array}{c}\text { Einfluss auf Streckenabschnitten } \\
\text { mit Anschlussknoten }\end{array}$} & \multicolumn{3}{|c|}{ Einfluss an lichtsignalgeregelten Knotenpunkte } \\
\hline & Unfallanzahl & Unfallschwere & Unfalltypen & Unfallanzahl & Unfallschwere & Unfalltypen \\
\hline RV-Stärke & MA, MO & MA & MA & $\mathrm{MI}$ & $\mathrm{Ml}$ & $\mathrm{MI}$ \\
\hline $\begin{array}{l}\text { Demografischer } \\
\text { Wandel }\end{array}$ & MA & MA & MA & MI & MA & MA, MI \\
\hline Geschwindigkeit & $M A, M O$ & MA & MA & MA & MA & MI \\
\hline
\end{tabular}

Legende:

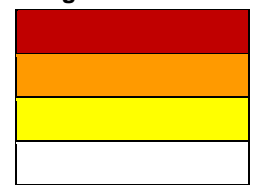

starker Einfluss

leichter Einfluss

ermittelter Einfluss, aber durch andere Einflussfaktoren überlagert

kein Einfluss vorhanden/ ermittelbar
Bewertungsgrundlagen:

MO - Unfallmodell

MI - mikroskopische Unfallanalyse

MA - makroskopische Unfallanalyse 


\section{Szenarien des künftigen Radverkehrs}

\subsection{Beschreibung der Szenarien}

In verschiedenen Szenarien wurden ausgehend von einem Nullszenario (SO - „Heutiger Radverkehr“) mögliche Entwicklungstendenzen des Radverkehrs entwickelt. In Anlehnung an aktuelle Tendenzen in der Bevölkerungsentwicklung, der Fahrradnutzung und der genutzten Fahrradtypen wurden begründete Annahmen für die folgenden Szenarien getroffen:

S1:

Kurzfristig moderate Zunahme des Radverkehrs

S2-A:

Mittelfristige Veränderung der Altersstruktur

S2-A-20: $\quad$ Veränderung der Altersstruktur und moderate Zunahme des Radverkehrs

S2-A-20-V: Veränderung der Geschwindigkeiten aufgrund einer veränderten Altersstruktur und veränderter Fahrradtypen bei moderater Zunahme des Radverkehrs

S2-A-40: Veränderung der Altersstruktur und erheblich höhere Stärken des Radverkehrs sowie

S2-A-40-V: Erheblich höhere Stärken und Veränderung der Geschwindigkeiten des Radverkehrs

Die Ausprägung der einzelnen Entwicklungstendenzen innerhalb der verschiedenen Szenarien wurden in Tabelle 39 zusammengefasst und werden im Folgenden erläutert.

Unter Zuhilfenahme der Ergebnisse der o. g. Analysen wurden anschließend künftige Entwicklungen für die Anzahl und die Schwere von Radverkehrsunfällen abgeschätzt (Kapitel 5.2 und 5.3). Ziel war dabei keine exakte Prognose, sondern ein Aufzeigen künftiger Entwicklungen der Radverkehrssicherheit, die sich in der heutigen Anzahl und Charakteristik von Radverkehrsunfällen begründen.

Tabelle 39: Übersicht der Entwicklungstendenzen in den Szenarien

\begin{tabular}{|c|c|c|c|c|c|c|c|c|}
\hline \multirow{2}{*}{ Szenario } & \multirow{2}{*}{ Zeithorizont } & \multicolumn{2}{|c|}{ Demografischer Wandel } & \multicolumn{3}{|c|}{$\begin{array}{c}\text { Radverkehrsanteil am } \\
\text { Modal Split }\end{array}$} & \multicolumn{2}{|c|}{$\begin{array}{c}\begin{array}{c}\text { Geschwindigkeiten im } \\
\text { Radverkehr (v) }\end{array} \\
\end{array}$} \\
\hline & & unverändert & mehr Ältere & $13 \%$ & $20 \%$ & $40 \%$ & unverändert & erhöht \\
\hline so & 2014 & $x$ & & $x$ & & & $x$ & \\
\hline S1 & 2020 & $x$ & & & $x$ & & $x$ & \\
\hline S2-A & \multirow{5}{*}{2030} & & \multirow{5}{*}{$x$} & $x$ & & & $x$ & \\
\hline S2-A-20 & & & & & \multirow{2}{*}{$x$} & & $x$ & \\
\hline S2-A-20-V & & & & & & & & $x$ \\
\hline S2-A-40 & & & & & & & $x$ & \\
\hline S2-A-40-V & & & & & & $\hat{n}$ & & $x$ \\
\hline
\end{tabular}




\section{S0: Heutiger Radverkehr}

Ein Nullszenario „heutiger Radverkehr“ war Ausganspunkt für die Entwicklungstendenzen der übrigen Szenarien. Bei einem Radverkehrsanteil von $13 \%$ an der Zahl der Wege wurden die in Tabelle 40 genannte Altersstruktur und mittlere Radfahrergeschwindigkeiten von $18,2 \mathrm{~km} / \mathrm{h}$ sowie eine $\mathrm{v}_{85}$ des Radverkehrs von $22,7 \mathrm{~km} / \mathrm{h}$ angesetzt.

Tabelle 40: Grundlagen des Szenarios S1 „Moderate Zunahme des Radverkehrs“

\begin{tabular}{|l|c|}
\hline \multicolumn{1}{|c|}{ Altersgruppe } & Anteil an der Bevölkerung (2009) [\%] ${ }^{\mathbf{4 1}}$ \\
\hline unter $\mathbf{1 0}$ & 8,4 \\
\hline $\mathbf{1 0 - 1 4}$ & 4,0 \\
\hline $\mathbf{1 5 - 1 7}$ & 2,6 \\
\hline $\mathbf{1 8 - 2 4}$ & 8,2 \\
\hline $\mathbf{2 5 - 6 5}$ & 57,5 \\
\hline $\mathbf{7 6 5}$ & 19,3 \\
\hline
\end{tabular}

\section{S1: Kurzfristig moderate Zunahme des Radverkehrs}

In einer Reihe von Groß- und Mittelstädten liegt der heutige Radverkehrsanteil am Gesamtverkehr bei etwa $20 \%$. Hierzu zählen z. B. Hannover (19\%), Kiel (21\%), Rostock (20\%) oder Bremen (25\%). Das Szenario unterstellte, dass der durchschnittliche Radverkehrsanteil in deutschen Kommunen von heute $13 \%$ auf zukünftig $20 \%$ steigt. $^{42}$

Der Szenariozeitraum erstreckt sich etwa bis in das Jahr 2020, Effekte einer veränderten Altersstruktur durch den demografischen Wandel kamen in diesem Szenario daher noch nicht zum Tragen.

Tabelle 41: Grundlagen des Szenarios S1 „Moderate Zunahme des Radverkehrs“

\begin{tabular}{|l|c|c|c|c|}
\hline \multirow{2}{*}{} & \multicolumn{3}{|c|}{ Radverkehrsanteil am Modal Split ${ }^{43}[\%]$} & \multirow{2}{*}{ Szenario } \\
\cline { 2 - 4 } & Ausgangslage & Annahme & relative Veränderung & Die Radverkehrsstärken steigen um $50 \%$. \\
\hline Fahrrad & $13^{44}$ & $\mathbf{2 0}$ & $+\mathbf{5 0} \%^{45}$ & . \\
\hline
\end{tabular}

\section{S2-A: Mittelfristige Veränderung der Altersstruktur}

Als Zeithorizont für dieses und die folgenden Szenarien wurde das Jahr 2030 angenommen. Mittelfristig ist eine Veränderung der Altersstruktur der Wohnbevölkerung Deutschlands zu erwarten. Als Grundlage dieses Szenarios wurde die Altersverteilung der 12. koordinierten Bevölkerungsvorausberechnung mit der Variante 1 - W2: Obergrenze der "mittleren" Bevölkerung für das Jahr 2030 herangezogen. Der Schwerpunkt des UDV-Projektes lag auf dem innerstädtischen Radverkehr. Herangezogen wurden daher die Bevölkerungsvorausberechnungen, wie sie das Statistische Bundesamt für die Stadtstaaten aufbereitet hat. Nach dieser Vorausberechnung steigt der Anteil von Über-65Jährigen an der Bevölkerung erheblich an (Tabelle 42).

41 Altersverteilung 2009 gemäß der 12. koordinierten Bevölkerungsvorausberechnung des Statistischen Bundesamtes für die Stadtstaaten. (Statistisches Bundesamt, 2009)

42 Ein entsprechend moderater Zuwachs des Radverkehrsanteils um $50 \%$ ist auch nach einem Forschungsvorhaben des Umweltbundesamtes möglich (Ahrens et al. 2013).

43 Modal Split auf Basis der Anzahl der Wege

44 SrV 2008 Gesamtverkehr

45 gerundet 
Bei diesem Szenario wurde unterstellt, dass mit der Veränderung der Altersanteile in der Bevölkerung auch die Veränderung der Anteile der Altersgruppen an den Radverkehrsstärken einhergeht. Der Radverkehrsanteil am Modal Split blieb in diesem Szenario im Vergleich zum Szenario S0 „heutiger Radverkehr" unverändert bei $13 \%$.

Tabelle 42: Grundlagen des Szenarios S2-A „Veränderung der Altersstruktur des Radverkehrs“

\begin{tabular}{|l|c|c|c|}
\hline \multirow{2}{*}{ Altersgruppe } & \multicolumn{2}{|c|}{ Anteil an der Bevölkerung [\%] } & \multirow{2}{*}{$\begin{array}{c}\text { relative Veränderung je Alters- } \\
\text { gruppe [\%] }\end{array}$} \\
\cline { 2 - 3 } & $\mathbf{2 0 0 9}$ & $\mathbf{2 0 3 0 ^ { 4 6 }}$ & -1 \\
\hline unter $\mathbf{1 0}$ & 8,4 & 8,3 & +10 \\
\hline $\mathbf{1 0 - 1 4}$ & 4,0 & 4,4 & -1 \\
\hline $\mathbf{1 5 - 1 7}$ & 2,6 & 2,6 & -23 \\
\hline $\mathbf{1 8 - 2 4}$ & 8,2 & 6,3 & -6 \\
\hline $\mathbf{2 5 - 6 5}$ & 57,5 & 53,9 & $\begin{array}{l}\text { Die Radverkehrsstärken der ver- } \\
\text { oder steigen entsprechend der links } \\
\text { genannten relativen Veränderungen. }\end{array}$ \\
\hline $\mathbf{5 6 5}$ & 19,3 & 24,5 & +27 \\
\hline
\end{tabular}

\section{S2-A-20: Veränderung der Altersstruktur und moderate Zunahme des Radverkehrs}

Dieses Szenario kombinierte die Veränderung der Altersstruktur wie bei S2-A und einen moderaten Zuwachs der Radverkehrsstärken um $50 \%$ wie in Szenario S1 (Tabelle 43).

Tabelle 43: Grundlagen des Szenarios S2-A-20 „Veränderung der Altersstruktur und moderate Zunahme des Radverkehrs“

\begin{tabular}{|l|c|c|c|}
\hline \multirow{2}{*}{ Altersgruppe } & \multicolumn{2}{|c|}{ Anteil an der Bevölkerung [\%] } & relative Veränderung je Alters- \\
gruppe [\%]
\end{tabular}

Die Vorausberechnung für die Stadtstaaten wurde beispielhaft auch um die Bevölkerungsprognosen für Dresden, Hannover, Leipzig, Münster und Stuttgart als Großstädte mittlerer Größe ergänzt. Diese lagen teils für das Jahr 2030 teils für das Jahr 2030 und mit örtlich teils anderen Abgrenzungen der Altersgruppen vor. Bei örtlich unterschiedlich starken Veränderungen waren auch in den meisten dieser Städte sinkende Anteile der Altersgruppe berufstätiger Erwachsener und höhere Anteile von Senioren zu erwarten. Auch für Kinder und Jugendliche war eine Zunahme ihres Bevölkerungsanteils zu erwarten (Schmitz-Veltin, 2009; Cicholas, U. et al., 2011; Bertelsmann Stiftung, 2014; LSK, 2011).

Vernachlässigt wurde die bundesweit von 81,7 Mio. (2009) auf 79 Mio. Einwohner (2030) sinkende Bevölkerung 
S2-A-20-V: Veränderung der Geschwindigkeiten aufgrund einer veränderten Altersstruktur und veränderter Fahrradtypen bei moderater Zunahme des Radverkehrs

Dieses Szenario kombinierte die Annahmen des Szenarios S2-A-20 mit einer Veränderung der genutzten Fahrradtypen auf folgender Grundlage:

Die Anteile der Pedelec 25-Nutzer am Radverkehr stiegen gegenüber den Zählungen im Jahr 2012 (unter $3 \%$ ) auf $20 \%$ bei Über-65-Jährigen und auf $15 \%$ bei 25- 64-Jährigen. Dies greift Untersuchungen aus den Niederlanden auf, nach denen

- derzeit $10 \%$ der niederländischen Wohnbevölkerung im Alter von über 60 Jahren, wie auch im Alter von 46 bis 60 Jahren, ein Pedelec 25 besitzen,

- nach Markteinschätzungen derzeit 50 \% der potentiellen Nutzer ein Pedelec 25 besitzen und

- künftig neben den „klassischen“ Nutzern der Über-65-Jährigen verstärkt Erwachsene mittlerer Altersgruppen ein Pedelec 25 nutzen (Van Boggelen et al, 2013)

Bei den o. g. Anteilen von Pedelec 25-Nutzern würden die Geschwindigkeiten des Gesamtradverkehrs um $0,4 \mathrm{~km} / \mathrm{h}$ steigen.

Tabelle 44: Grundlagen des Szenarios S2-A-20-V „Veränderung der Geschwindigkeiten aufgrund einer veränderten Altersstruktur und veränderter Fahrradtypen bei moderater Zunahme der Radverkehrsstärken“

\begin{tabular}{|c|c|c|c|c|c|c|c|}
\hline \multirow[t]{2}{*}{ Altersgruppe } & \multirow{2}{*}{$\begin{array}{l}\text { relative Verände- } \\
\text { rung der Personen } \\
\text { in den Altersgrup- } \\
\text { pen } 2009-2030 \text { [\%] }\end{array}$} & \multicolumn{2}{|c|}{$\begin{array}{l}\text { Anteil Pedelec 25-Nutzer [\%] } \\
\text { am Radverkehr }\end{array}$} & \multicolumn{2}{|c|}{$\begin{array}{l}\text { Geschwindigkeiten } \\
\text { des künftigen Radver- } \\
\text { kehrs }\end{array}$} & \multirow{2}{*}{$\begin{array}{c}\text { Modal Split- } \\
\text { Anteil Radver- } \\
\text { kehr Zahl der } \\
\text { Wege [\%] } \\
\begin{array}{c}\text { relative Verän- } \\
\text { derung }\end{array}\end{array}$} & \multirow[t]{2}{*}{ Szenario } \\
\hline & & $\begin{array}{c}\text { Zählungen } \\
2012\end{array}$ & 2030 & $v_{m}$ & $v_{85}$ & & \\
\hline unter 10 & -1 & & & \multirow{6}{*}{$+0,4 \mathrm{~km} / \mathrm{h}$} & \multirow{6}{*}{$+0,4 \mathrm{~km} / \mathrm{h}$} & \multirow{6}{*}{$+50 \%$} & \multirow{6}{*}{$\begin{array}{l}\text { Die Radverkehrsstärken } \\
\text { steigen um } 50 \% \text {. } \\
\text { Die } v_{m} \text { und die } v_{85} \text { des } \\
\text { Gesamtradverkehrs } \\
\text { steigen um } 0,4 \mathrm{~km} / \mathrm{h} \text {. }\end{array}$} \\
\hline $10-14$ & +10 & & & & & & \\
\hline $15-17$ & -1 & & & & & & \\
\hline $18-24$ & -23 & & & & & & \\
\hline $25-65$ & -6 & 0,2 & 15 & & & & \\
\hline$>65$ & +27 & 2,2 & 20 & & & & \\
\hline
\end{tabular}




\section{S2-A-40: Veränderung der Altersstruktur und erheblich höhere Stärken des Radverkehrs}

In Münster beträgt der heutige Radverkehrsanteil am Gesamtverkehr 38 \% (2007). Das Szenario setzte vergleichbar hohe Radverkehrsanteile am Gesamtverkehr auch für andere Städte und eine Veränderung der Altersstruktur wie in Szenario S2-A an (Tabelle 45, Tabelle 46). ${ }^{47}$

Tabelle 45: Grundlagen „Erheblich höhere Stärken des Radverkehrs“

\begin{tabular}{|c|c|c|c|}
\hline \multicolumn{2}{|c|}{ Radverkehrsanteil am Modal Split ${ }^{48}[\%]$} & \multirow{2}{*}{ relative Veränderung } & \multirow{2}{*}{ Szenario } \\
\cline { 1 - 2 } Ausgangslage & Annahme & $+200 \%$ & Die Radverkehrsstärken steigen um $200 \%$. \\
\hline $13^{\text {Fenler! Textmarke nicht detiniert. }}$ & $40^{47}$ & +2000 \\
\hline
\end{tabular}

Tabelle 46: Grundlagen des Szenarios S2-A-40 „Veränderung der Altersstruktur und erheblich höhere Stärken des Radverkehrs“

\begin{tabular}{|c|c|c|c|c|}
\hline \multirow{2}{*}{ Altersgruppe } & \multicolumn{2}{|c|}{ Anteil an der Bevölkerung [\%] } & \multirow{2}{*}{$\begin{array}{l}\text { relative Veränderung je } \\
\text { Altersgruppe [\%] }\end{array}$} & \multirow{2}{*}{ Szenario } \\
\hline & 2009 & $2030^{46}$ & & \\
\hline unter 10 & 8,4 & 8,3 & -1 & \multirow{6}{*}{$\begin{array}{l}\text { Die Radverkehrsstärken der verschiedenen Alters- } \\
\text { gruppen sinken oder steigen entsprechend der links } \\
\text { genannten relativen Veränderungen. Die Radver- } \\
\text { kehrsstärken steigen insgesamt um } 200 \% \text {. }\end{array}$} \\
\hline $10-14$ & 4,0 & 4,4 & +10 & \\
\hline $15-17$ & 2,6 & 2,6 & -1 & \\
\hline $18-24$ & 8,2 & 6,3 & -23 & \\
\hline $25-65$ & 57,5 & 53,9 & -6 & \\
\hline$>65$ & 19,3 & 24,5 & +27 & \\
\hline
\end{tabular}

\section{S2-A-40-V: Erheblich höhere Stärken und Veränderung der Geschwindigkeiten des Radver- kehrs}

Grundlagen dieses Szenarios waren erheblich höhere Stärken des Radverkehrs und eine mittelfristige Bevölkerungsentwicklung entsprechend S2-A-40 sowie eine Veränderung der Geschwindigkeiten durch eine steigende Pedelec-Nutzung entsprechend S2-A-20-V (Tabelle 47).

Tabelle 47: Grundlagen des Szenarios S2-A-40-V „Erheblich höhere Stärken und Veränderung der Geschwindigkeiten des Radverkehrs"

\begin{tabular}{|c|c|c|c|c|c|}
\hline \multirow[t]{2}{*}{ Altersgruppe } & \multirow[t]{2}{*}{$\begin{array}{c}\text { relative Veränderung } \\
2009-2030[\%]\end{array}$} & \multicolumn{2}{|c|}{$\begin{array}{l}\text { Anteil Pedelec 25-Nutzer [\%] } \\
\text { am Radverkehr }\end{array}$} & \multirow{2}{*}{$\begin{array}{c}\text { Modal Split-Anteil Rad- } \\
\text { verkehr Zahl der Wege } \\
\text { [\%] } \\
\text { relative Veränderung }\end{array}$} & \multirow[t]{2}{*}{ Szenario } \\
\hline & & Zählungen 2012 & künftig & & \\
\hline unter 10 & -1 & & & \multirow{6}{*}{$+200 \%$} & \multirow{6}{*}{$\begin{array}{l}\text { Die Radverkehrsstärken } \\
\text { steigen um } 200 \% \text {. } \\
\text { Die } v_{\mathrm{m}} \text { und die } \mathrm{v}_{85} \text { des Ge- } \\
\text { samtradverkehrs steigt um } \\
0,4 \mathrm{~km} / \mathrm{h} \text {. }\end{array}$} \\
\hline $10-14$ & +10 & & & & \\
\hline $15-17$ & -1 & & & & \\
\hline $18-24$ & -23 & & & & \\
\hline $25-65$ & -6 & 0,2 & 15 & & \\
\hline$>65$ & +27 & 2,2 & 20 & & \\
\hline
\end{tabular}

47 Nach Ahrens et al. (2013) sind noch höhere Radverkehrsanteile von 49 \% am Gesamtverkehr möglich.

48 Modal Split auf Basis der Anzahl der Wege 


\subsection{Auswirkungen der Szenarioannahmen auf die Verkehrssicherheit}

\subsubsection{Streckenabschnitte}

\section{Unfallanzahl und Unfallschwere}

Die Auswirkungen der in Kap. 5.1 als Szenarien angenommenen Veränderungen auf die Verkehrssicherheit des Radverkehrs wurden mit Hilfe der Ergebnisse der Makroanalyse, des Unfallmodells und der strecken- und nutzerbezogenen Daten quantifiziert. Die einzelnen Szenarien unterschieden sich in den Kombinationen der Ausprägung der Kriterien:

- Zeithorizont,

- demografischer Wandel,

- Radfahreranteil und

- im Radverkehr gefahrene Geschwindigkeiten.

Diese Kriterien blieben je nach Szenario jeweils unverändert oder verändern sich in unterschiedlichem Maß. Der Einfluss jedes einzelnen Kriteriums wurde in den einzelnen Schritten der Berechnung des zukünftigen Unfallgeschehens ermittelt.

Die Veränderungen der Radverkehrsstärken und der demografischen Struktur bewirkten sowohl eine Veränderung in der Unfallanzahl, als auch in der Unfallschwere. Im Gegensatz dazu beeinflusste die Geschwindigkeit nur die Unfallschwere. In einem separaten Unfallmodell (Tabelle 36) konnte ein Zusammenhang zwischen der Geschwindigkeit und der Unfallanzahl hergestellt werden, jedoch zeigten die makroskopischen Untersuchungen, dass dieser Effekt nicht getrennt betrachtet werden konnte von dem Zusammenhang zwischen der Radverkehrsstärke und den gefahrenen Geschwindigkeiten (Bild 56). Der Einfluss der Geschwindigkeit auf die Unfallanzahl wurde bei der Berechnung der Auswirkung der Szenarien demnach nicht direkt berechnet, sondern gewissermaßen indirekt über den Einfluss der Radverkehrsstärken auf die Unfallanzahl berücksichtigt (siehe Kapitel 4.4.1).

Bild 62 zeigt die prinzipielle Vorgehensweise und die einzelnen Schritte bei der Quantifizierung des zukünftigen Unfallgeschehens. Je nach Kriterienkombination der einzelnen Szenarien konnten einzelne Schritte entfallen ${ }^{49}$.

49 In Abhängigkeit der Szenarien ergab sich die Notwendigkeit die Aufteilung der Unfallschwere in mehreren Schritten zu berechnen. Dazu wurden in der Berechnung jeweils eine modifizierte Unfallanzahl berechnet und je nach Kombination der Veränderung von DTV, Radverkehrsalter und Geschwindigkeiten bedingte Wahrscheinlichkeiten verwendet. 


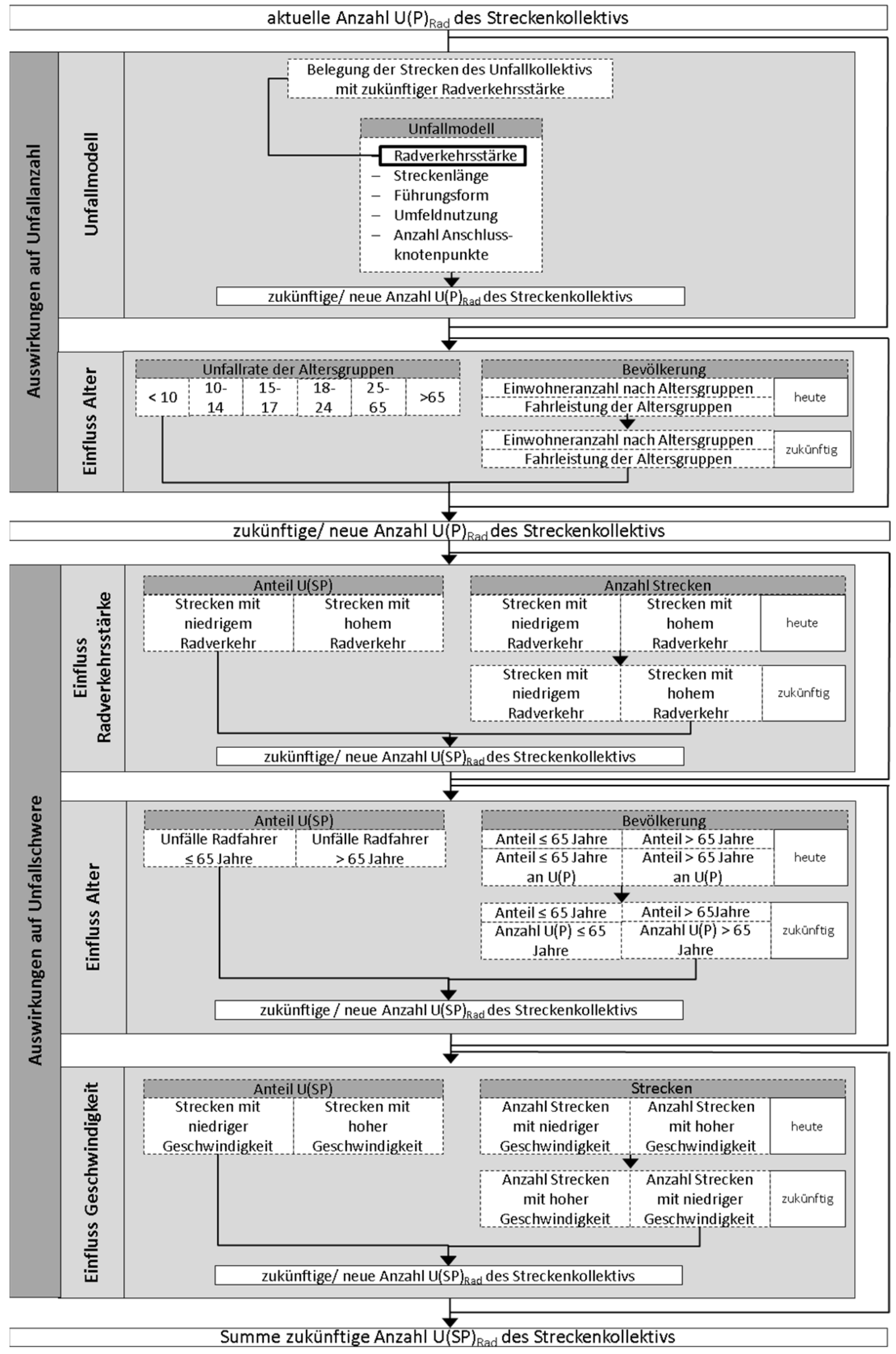

Bild 62: Ablauf der Szenarienberechnungen für Strecken 
In den einzelnen Schritten wurde wie folgt vorgegangen.

\section{Einfluss des DTVs auf die Unfallanzahl (Unfallmodell)}

Im Unfallmodell wurde zunächst berechnet, wie viele $U(P)_{\text {Rad }}$ im jeweiligen Szenario zu erwarten waren. Das Ergebnis wurde dabei bestimmt von:

- den Formen der Radverkehrsanlagen im Streckenkollektiv (diese bleiben für alle Szenarien unverändert) und

- der Entwicklung des weiteren Kriteriums „Radfahreranteil“.

Anhand der Entwicklung des Radfahreranteils bestimmte sich die Veränderung der Radverkehrsstärke. Eine Zunahme des Radfahreranteils auf $20 \%$ (von heute ca. $13 \%$ ) führt zu einer Veränderung der Radverkehrsstärken um 50 \%; die Zunahme des Radverkehrsanteils auf $40 \%$ zu einer Veränderung der Radverkehrsstärken um 200 \%. Je nach Ausprägung des zukünftigen Radfahreranteils wurden die Radverkehrsstärken aller Strecken im Kollektiv (unabhängig der Führungsform des Radverkehrs) um diese Werte verändert. Unter der Annahme, dass sich die Umfeldnutzung (Gewerbe/ Geschäfte), der Typ der Radverkehrsanlage und die Anzahl der Anschlussknotenpunkte nicht ändern, wurde die zukünftige Anzahl an $U(P)_{\text {Rad }}$ mittels der neuen Radverkehrsstärke errechnet. Die Zuschläge, die im Modell für einzelne Formen der Radverkehrsanlagentypen bestimmt wurden, blieben unverändert. Zukünftige Verlagerungseffekte auf andere Führungsformen des Radverkehrs infolge erhöhter Radverkehrsstärken blieben unberücksichtigt. Dies folgte aus der Annahme, dass die Verteilung der einzelnen Führungsformen des Radverkehrs im Streckenkollektiv repräsentativ ist.

\section{Einfluss des Alters auf die Unfallanzahl}

Auf Basis altersgruppenspezifischer Unfallraten aus der makroskopischen Unfallanalyse (Kapitel 4.3.1) wurde die Veränderung der zukünftigen Unfallanzahl errechnet.

Das Ergebnis wurde dabei bestimmt von:

- der Aufteilung der Bevölkerung auf die Altersgruppen,

- der Fahrleistung der Altersgruppen nach MiD 2008 und

- der altersspezifischen Unfallrate.

Aus der Aufteilung der Bevölkerung auf die Altersgruppen und der Fahrleistung der Altersgruppen ließ sich die altersspezifische Radfahrleistung je Einwohner ermitteln. Mit den Annahmen zur Verschiebung der Bevölkerungsanteile in den Altersgruppen, wurden die zukünftigen Fahrleistungen und Fahrleistungsanteile der einzelnen Altersgruppen berechnet. In der Berechnung wurde von unveränderten Unfallraten und Fahrleistungen je Einwohner in den Altersgruppen ausgegangen.

\section{Einfluss der Radverkehrsstärke auf die Unfallschwere}

In diesem Schritt wurde die Aufteilung der Radverkehrsunfälle mit schwerem Personenschaden (im Folgenden: schwere Radverkehrsunfälle) und leichtem Personenschaden unter Betrachtung des Einflusses höherer Radverkehrsstärken bestimmt. Die Berechnung richtete sich dabei nach

- den Radverkehrsstärken der einzelnen Strecken im Kollektiv und

- der Entwicklung des Radverkehrsanteils.

Die makroskopische Analyse zeigte, dass auf Strecken mit hohen Radverkehrsstärken im Mittel auch der Anteil schwerer Radverkehrsunfälle an allen Radverkehrsunfällen mit Personenschaden höher lag (vgl. Kapitel 4.2.2). 
Die auf den 200 Untersuchungsstrecken gemessenen Radverkehrsstärken wurden je nach Szenario um 50 bzw. $200 \%$ erhöht. Entsprechend den modifizierten Radverkehrsstärken auf den Untersuchungsstrecken wurde der neue Anteil der schweren Radverkehrsunfälle abgeleitet.

\section{Einfluss des Alters auf die Unfallschwere}

In den Szenarien zum Jahr 2030 wurde von einer demografischen Verschiebung ausgegangen, die eine Steigerung des Anteils von Über-65-Jährigen an der Bevölkerung von heute 19,3\% auf 24,5\% annimmt. Die makroskopische Analyse zeigte, dass in der Altersgruppe der Über-65-Jährigen der Anteil schwerer Radverkehrsunfälle an allen Radverkehrsunfällen mit Personenschaden höher lag. Unter der Annahme gleichbleibender Unfallraten ergaben sich Veränderungen der Unfallanzahl jedoch insgesamt erst bei einer deutlichen Verschiebung der Anzahl der Personen innerhalb der maßgebenden Altersgruppen (vgl. 4.3.2 und Bild 50).

In diesem Schritt wurde der Einfluss eines erhöhten Anteils an Über-65-Jährigen im Radverkehr auf die Aufteilung der Radverkehrsunfälle mit schwerem Personenschaden und leichtem Personenschaden bestimmt. Die Berechnung richtete sich dabei nach der Veränderung der Beteiligung der Über65-Jährigen am Radverkehr.

In den Berechnungen wird davon ausgegangen, dass sich:

- die Beteiligung der Altersgruppen am Radverkehr und

- deren Unfallrisiko

in der Zukunft nicht verändern.

Auf Basis der zukünftigen demografischen Struktur wurden die Verteilung der Unfälle auf die Altersgruppen und deren spezifischer Anteil an schweren Radverkehrsunfällen an allen Radverkehrsunfällen mit Personenschaden bestimmt.

\section{Einfluss der Geschwindigkeit auf die Unfallschwere}

In den Szenarien zum Jahr 2030 wurde von einer stärkeren Nutzung von Pedelec 25 ausgegangen. Von heute unter $3 \%$ Anteil im Bestand wird der Anteil in der Altersgruppe der Unter-65-Jährigen in den Szenarien S2-A-20-V und S2-A-40-V auf $15 \%$ und in der Altersgruppe der Über-65-Jährigen auf $20 \%$ ansteigen.

In diesem Schritt wurde der Einfluss eines erhöhten Geschwindigkeitsniveaus auf die Aufteilung der Radverkehrsunfälle mit schwerem Personenschaden und leichtem Personenschaden bestimmt. Die Berechnung richtete sich dabei nach dem Geschwindigkeitsniveau der einzelnen Strecken im Kollektiv.

Die makroskopische Analyse zeigte, dass auf Strecken mit hohen Geschwindigkeiten im Mittel auch der Anteil schwerer Radverkehrsunfälle an allen Radverkehrsunfällen mit Personenschaden höher lag (Bild 58).

Die auf den 200 Untersuchungsstrecken gemessenen Geschwindigkeiten wurden um 0,4 km/h erhöht. Entsprechend dem Einfluss dieses veränderten Geschwindigkeitsniveaus auf den Untersuchungsstrecken wurde der neue Anteil der schweren Radverkehrsunfälle abgeleitet.

\section{Verteilung der Radverkehrsunfälle auf die verschiedenen Führungsformen des Radverkehrs}

Die Untersuchungen in Kap. 4.2.1 haben sowohl im Unfallmodell als auch der makroskopischen Unfalluntersuchung gezeigt, dass sich das Unfallgeschehen auf Streckenabschnitten, je nach Führungsform der Radverkehrsanlage, in unterschiedlichem Maße mit dem Anstieg der Radverkehrsstärke änderte. Daher ist bei unveränderter zukünftiger Radverkehrsinfrastruktur (bzw. Gegenmaßnahmen) mit einer anteiligen Verschiebung der Unfälle auf die Führungsformen zu rechnen. Anhand des Stre- 
ckenkollektivs wurde unter der Annahme steigender Radverkehrsstärken die anteilige Verschiebung ermittelt.

Da das Streckenkollektiv repräsentativ für die Aufteilung der einzelnen Führungsformen des Radverkehrs war und angenommen wurde, dass die Veränderungen der Radverkehrsstärken gleichmäßig an allen Radverkehrsanlagentypen auftraten, wurde die Anzahl der Radverkehrsunfälle zunächst über das Unfallmodell und die Neuverteilung der Unfallschwere aus den Erkenntnissen der makroskopischen Untersuchung berechnet (siehe Bild 63). Die Berechnungen basierten auf den Ergebnissen der makroskopischen Unfallanalyse, weshalb die Verteilung der Unfälle auf die dort untersuchten Führungsformen berechnet (Schutzstreifen, Radweg, und Radfahrstreifen, Führung auf der Fahrbahn) wurde.

Um die Veränderungen der demografischen Struktur einzubeziehen, erfolgte eine Anpassung der jeweiligen Unfallzahlen analog zu den Ausführungen in 5.2.1. Da kein Zusammenhang der Führungsform auf das Unfallgeschehen einzelner Altersgruppen nachgewiesen werden konnte, bewirkt eine Änderung der demografischen Struktur keine anteilige Veränderung der Unfallaufteilung auf die Führungsformen.

Der Einfluss der Geschwindigkeit auf die Unfallanzahl wurde durch den Einfluss der Radverkehrsstärken dargestellt (siehe Erläuterungen in 5.2.1).

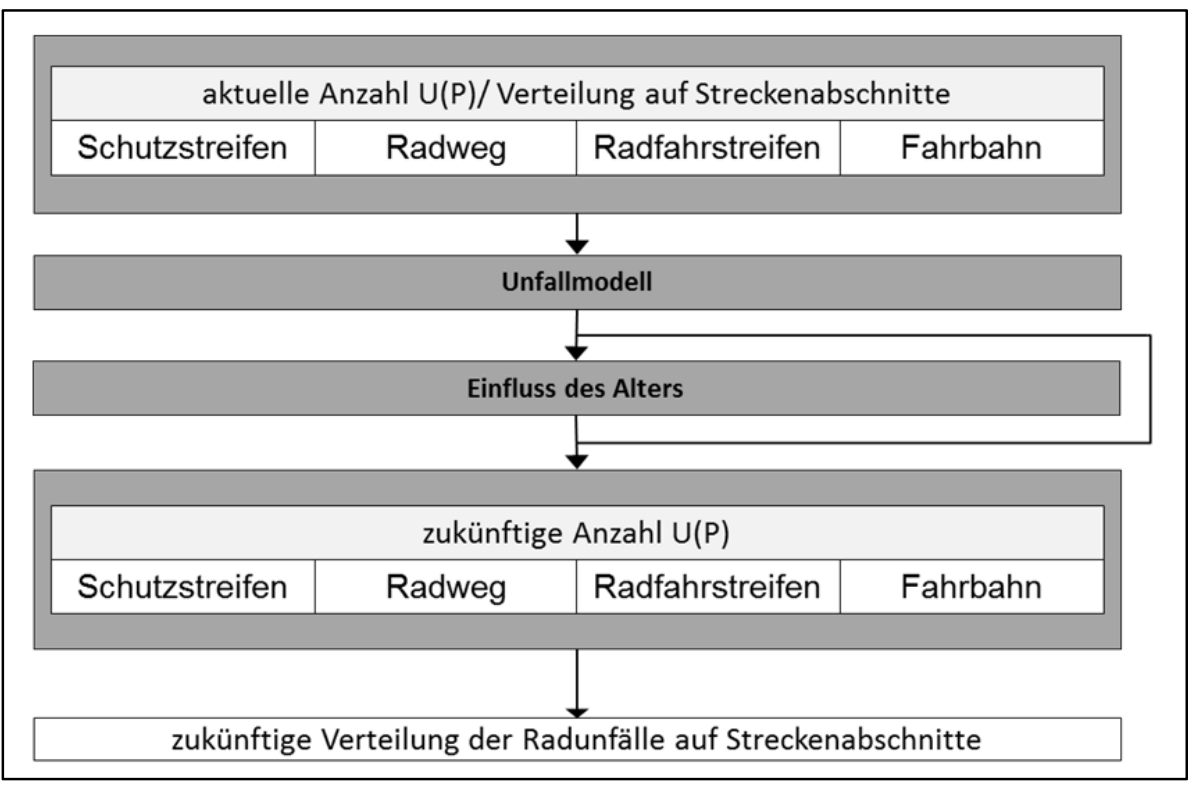

Bild 63: Inhaltlicher Ablauf der Szenarienberechnungen zur Aufteilung der Unfälle auf die Streckenabschnitte

\section{Entwicklung der Unfalltypen auf den Streckenabschnitten}

Die Vorgehensweise zur Bestimmung der Unfallanzahl und -schwere in den jeweiligen Szenarien diente auch zur Bestimmung der Verteilung der Unfalltypen im Kollektiv (Bild 62).

Die Schätzung der zu erwartenden Unfallanzahl wurde daher aus den Szenarienberechnungen zur Unfallanzahl und -schwere auf den Streckenabschnitten übernommen.

Anhand des Streckenkollektivs wurden schrittweise die Einflüsse der veränderten Radverkehrsstärke und Rad-Geschwindigkeiten auf die Unfalltypenverteilung ermittelt.

Auf Basis der zukünftigen demografischen Struktur wurde die Verteilung der Unfälle auf die Altersgruppen und deren spezifischer Anteile der Unfalltypen bestimmt.

Für Szenarien mit erhöhten Geschwindigkeitsniveaus (S2-A-20-V und S2-A-40-V) waren die gemessenen Geschwindigkeiten auf den Untersuchungsstrecken um 0,4 km/h erhöht. Entsprechend dem 
Einfluss dieses veränderten Geschwindigkeitsniveaus auf den Untersuchungsstrecken wurde der neue Anteil der Unfalltypen abgeleitet.

Analog wurden in den Szenarien mit höherer Radverkehrsstärke die gemessenen Radverkehrsstärken auf den Untersuchungsstrecken um 50 \% (S1, S2-A-20, S2-A-20-V) bzw. 200 \% (S2-A-40, S2-A40-V) erhöht und der neue Anteil der Unfalltypen bestimmt.

Die Berechnungen gingen von einer unveränderten Infrastruktur (Art der Radverkehrsanlage, Umfeldnutzung, Anzahl der Anschlussknotenpunkte) aus. Je nach Kriterienkombination der einzelnen Szenarien konnten einzelne Schritte entfallen.

\subsubsection{Lichtsignalgeregelte Knotenpunkte}

\section{Einfluss von Radverkehrsstärken auf die Unfallanzahl und die Unfallschwere}

In der Mikroanalyse hatte sich gezeigt, dass kein Einfluss das Radverkehrsaufkommen auf die Unfallanzahl und die Unfallschwere nachgewiesen werden konnte. Die Unfallanzahl und der Anteil der Unfälle mit schwerem Personenschaden wurden daher für den Fall steigender Radverkehrsstärken als unverändert angesetzt.

\section{Einfluss des Alters}

Nach den Ergebnissen der Mikroanalyse beeinflusste die Altersstruktur im Hinblick auf die Anteile der Über-65-jährigen Radfahrer die Unfallanzahl an lichtsignalgeregelten Knotenpunkten nicht. Demgegenüber hatte die Makroanalyse eine höhere Gefährdung von Über-65-Jährigen im Hinblick auf Unfälle mit schwerem Personenschaden ermittelt (Anteil schwerer Unfälle an Unfällen mit Personenschaden bei Über-65-Jährigen $27 \%$, bei Unter-65-Jährigen $14 \%$ ).

Zur Berechnung der Entwicklung schwerer Unfälle wurde daher zum einen die in der Makroanalyse ermittelte jeweilige Unfallanzahl der Altersgruppen auf einen zukünftig höheren Anteil Über-65Jähriger bezogen. ${ }^{50}$ Auf Basis der zukünftigen demografischen Struktur wurden die Verteilung der Unfälle auf die Altersgruppen und der Anteil von schweren Radverkehrsunfällen an allen Radverkehrsunfällen mit Personenschaden bestimmt. Hierbei wurde davon ausgegangen, dass sich

- die Beteiligung der Altersgruppen am Radverkehr und

- deren Unfallrisiko

in der Zukunft nicht verändern.

Vergleichbare Anteile schwerer Unfälle hatten sich in der Makro- als auch sich in der Mikroanalyse gezeigt. ${ }^{51}$ Vergleichend wurde daher die in der Mikroanalyse ermittelte Altersstruktur des in die Knotenpunkte einfahrenden Radverkehrs mit einem zukünftig höheren Anteil Über-65-Jähriger herangezogen und die künftigen Anteile schwerer Unfälle berechnet.

\footnotetext{
$50 \quad$ alle Unfälle mit Radbeteiligung

51 Anteil schwerer Unfälle im Knotenkollektiv der Mikroanalyse $26 \%$ bei Über-65-Jährigen und $15 \%$ bei Unter-65Jährigen.
} 


\section{Einfluss der Geschwindigkeiten}

Bei steigenden Geschwindigkeiten war eine weitere Zunahme der Unfallanzahl möglich, diese war wegen eines sehr kleinen Kollektivs an Unfällen mit der Ursache "Geschwindigkeit" aber nicht quantifizierbar.

\section{Verteilung der Radverkehrsunfälle auf die verschiedenen Führungsformen des Radverkehrs}

Anders als für die Streckenabschnitte zeigte sich für die lichtsignalisierten Knotenpunkte kein Einfluss der Radverkehrsstärken auf die Unfallanzahl. Die insgesamt niedrige Anzahl von Unfällen Über-65Jähriger sowie geschwindigkeitsbeeinflusster Unfälle, die in der Mikroanalyse betrachtet werden konnten, ließen keine Verallgemeinerung für alters- oder geschwindigkeitsbedingte Gefährdungen auf bestimmten Anlagentypen an lichtsignalisierten Knotenpunkten zu. Eine Veränderung der Verteilung auf die verschiedenen Führungsformen wurde daher hier nicht berechnet.

\section{Entwicklung der Unfalltypen an lichtsignalgeregelten Knotenpunkten}

Die Makroanalyse hatte für lichtsignalgeregelte Knotenpunkte höhere Anteile von Einbiegen-/ Kreuzen-Unfällen für Über-65-Jährige ermittelt (Anteil Einbiegen-/ Kreuzen-Unfälle bei Über-65-Jährigen $44 \%$, bei Unter-65-Jährigen $40 \%$ ). Da die Radverkehrsstärken keine belastbaren Einflüsse auf die Unfalltypen zeigten und nur einzelne Knotenpunkte Hinweise auf geschwindigkeitsbedingte Einflüsse gaben, wurde lediglich die altersbedingte Entwicklung der Unfalltypen betrachtet.

\subsubsection{Gesamtunfallgeschehen}

Anschließend wurde die Entwicklung der Unfälle an Streckenabschnitten und lichtsignalgeregelten Knotenpunkten vergleichend gegenübergestellt. Auf Grundlage der Verteilung der Unfälle des Anlagenkollektivs dieser Untersuchung wurden die Anteile der auf Strecken und Knotenpunkte entfallenden Unfälle für die einzelnen Szenarien berechnet. Die Berechnung erfolgte dabei für ein normiertes Kollektiv von jeweils 200 Streckenabschnitte und Knotenpunkte. 


\subsection{Ergebnisse der Szenarienberechnungen}

\subsubsection{Streckenabschnitte}

\section{Unfallanzahl und Unfallschwere}

Die Ergebnisse der Szenarienberechnungen ergaben sich aus den unterschiedlichen Einflüssen durch die Veränderung des DTV, der Geschwindigkeit und der Altersstruktur. Tabelle 48 zeigt die Gegenüberstellung der Ergebnisse im Hinblick auf:

- die Zunahme der Anzahl der Unfälle mit Personenschaden,

- die Zunahme der Anzahl der Radverkehrsunfälle mit schwerem Personenschaden und

- den daraus resultierenden Anteil der schweren Radverkehrsunfälle an allen Radverkehrsunfällen mit Personenschaden.

Tabelle 48: Ergebnisse der Szenarienberechnung zur Unfallschwere an Streckenabschnitten

\begin{tabular}{|c|c|c|c|c|c|c|c|c|c|c|c|}
\hline \multirow[b]{2}{*}{ Szenario } & \multirow[b]{2}{*}{$\begin{array}{c}\text { Zeit- } \\
\text { horizont }\end{array}$} & \multicolumn{2}{|c|}{$\begin{array}{c}\text { demogra- } \\
\text { fischer Wan- } \\
\text { del } \\
\end{array}$} & \multicolumn{3}{|c|}{$\begin{array}{c}\text { Radverkehrsanteil } \\
\text { am Modal Split }\end{array}$} & \multicolumn{2}{|c|}{$\begin{array}{l}\text { Geschwin- } \\
\text { digkeit (v) }\end{array}$} & \multirow[b]{2}{*}{$\begin{array}{c}\text { Zunahme Unfallanzahl } \\
\mathrm{U}(\mathrm{P})_{\mathrm{Rad}}[\%]\end{array}$} & \multirow[b]{2}{*}{$\begin{array}{c}\text { Zunahme } \\
\text { U(SP) Rad [\%] }\end{array}$} & \multirow[b]{2}{*}{$\begin{array}{c}\text { Anteil } \\
\mathrm{U}(\mathrm{SP})_{\text {Rad }}[\%\end{array}$} \\
\hline & & 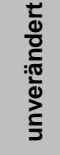 & 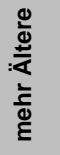 & $\stackrel{\circ}{\stackrel{0}{9}}$ & ஃ̊ & $\stackrel{\circ}{\stackrel{q}{q}}$ & 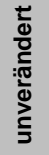 & 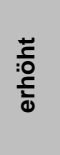 & & & \\
\hline so & 2014 & $x$ & & $x$ & & & $x$ & & \pm 0 & 0 & 14 \\
\hline S1 & 2020 & $x$ & & & $x$ & & $x$ & & 29 & 36 & 14 \\
\hline S2-A & \multirow{5}{*}{2030} & & \multirow{5}{*}{$x$} & $x$ & & & $x$ & & 1 & 3 & 14 \\
\hline S2-A-20 & & & & & \multirow{2}{*}{$x$} & & $x$ & & 31 & 40 & 15 \\
\hline S2-A-20-V & & & & & & & & $x$ & 31 & 43 & 15 \\
\hline S2-A-40 & & & & & & Y & $x$ & & 102 & 136 & 16 \\
\hline S2-A-40-V & & & & & & $x$ & & $x$ & 102 & 140 & 16 \\
\hline
\end{tabular}

In den Szenarien, die von einem Anstieg des Radverkehrsanteils auf $20 \%$ ausgingen, bewirkte das eine Zunahme der Radverkehrsstärke um 50 \%. Diese Zunahme bewirkte einen Anstieg der Unfallzahlen um $29 \%$ im Vergleich zum Szenario S0 (heutiger Radverkehr). Wurde neben dem Anstieg der Radverkehrsstärke von einer Veränderung der demografischen Struktur ausgegangen, erhöhte sich die Unfallanzahl um $31 \%$. Analog ergab der Anstieg des Anteils der zurückgelegten Wege mit dem Rad auf $40 \%$ eine Zunahme der Radverkehrsstärke um $200 \%$ und somit einen Anstieg der Unfallzahlen um $102 \%$. Der Einfluss durch die veränderte demografische Struktur bewirkte keine wesentliche Zunahme der Unfallzahl auf $102 \%$.

Der Anstieg der schweren Radverkehrsunfälle wurde im Wesentlichen vom Anstieg der Radverkehrsstärken (über die Zunahme aller Radverkehrsunfälle bei gleichzeitig zunehmendem Anteil der schweren Radverkehrsunfälle) bestimmt. Bei einem Anstieg der Radverkehrsstärken um 50 \% stiegen die schweren Radverkehrsunfälle um 36 \% (Szenario S1). Die schweren Radverkehrsunfälle stiegen dabei in einem stärkeren Maße an als die Gesamtanzahl der Radverkehrsunfälle mit Personenschaden.

Im Hinblick auf die Anzahl schwerer Radverkehrsunfälle ergaben sich durch die Veränderungen in der demografischen Struktur ca. 3\% mehr Radverkehrsunfälle mit schwerem Personenschaden (vgl. S2-A und S0 in Tabelle 48). Aus dem Anstieg der Radverkehrsgeschwindigkeiten resultierte ein Anstieg der schweren Radverkehrsunfälle um ca. 3-4 Prozentpunkte (vgl. S2-A-20 und S2-A-20-V).

Zusammengefasst ist festzuhalten, dass in Zukunft die Anzahl der Radverkehrsunfälle mit Personenschaden und noch stärker die Anzahl der Radverkehrsunfälle mit schwerem Personenschaden, vor allem bedingt durch den Anstieg der Radverkehrsstärken, stark zunehmen werden, wenn keine geeigneten Gegenmaßnahmen ergriffen werden. Unter den „ungünstigsten“ Bedingungen (Szenario S2-A-40-V) wird sich die Anzahl der Radverkehrsunfälle mit Personenschaden unter den 
angenommenen Bedingungen mehr als verdoppeln und sich die Anzahl der schweren Radverkehrsunfälle im Vergleich zu den heutigen Zahlen um den Faktor 2,4 erhöhen.

\section{Verteilung der Radverkehrsunfälle auf die verschiedenen Führungsformen des Radverkehrs}

Die Anzahl und Verteilung der jeweiligen Führungsformen im Bestand hatten maßgeblichen Einfluss auf die Verteilung der Unfälle. Anzahl und Verteilung der Führungsformen blieben bei diesen Betrachtungen über alle Szenarien unverändert.

In der makroskopischen Untersuchung hatten auf den Streckenabschnitten sowohl die Führung des Radverkehrs auf der Fahrbahn als auch auf dem Radfahrstreifen die stärkste Beeinflussung der Unfallzahlen durch die Radverkehrsstärke gezeigt (Bild 16). Für diese Führungsformen war daher auch in den Szenarien mit Anstieg der Radverkehrsstärke die stärkste Zunahme an Radverkehrsunfällen auf den Streckenabschnitten zu verzeichnen. Daraus resultierte in der Summe eine verstärkte Verlagerung der Unfälle auf diese Führungsformen. Je nach Entwicklung des Radverkehrsanteils auf $20 \%$ bzw. 40 \% stieg der Anteil der Radverkehrsunfälle mit Personenschaden auf diesen Führungsformen um 3 bzw. 6 Prozentpunkte. Auf Schutzstreifen blieb der Anteil annähernd gleich, sodass die geringsten Zunahmen auf Radwegen zu verzeichnen waren (vgl. Bild 64). 


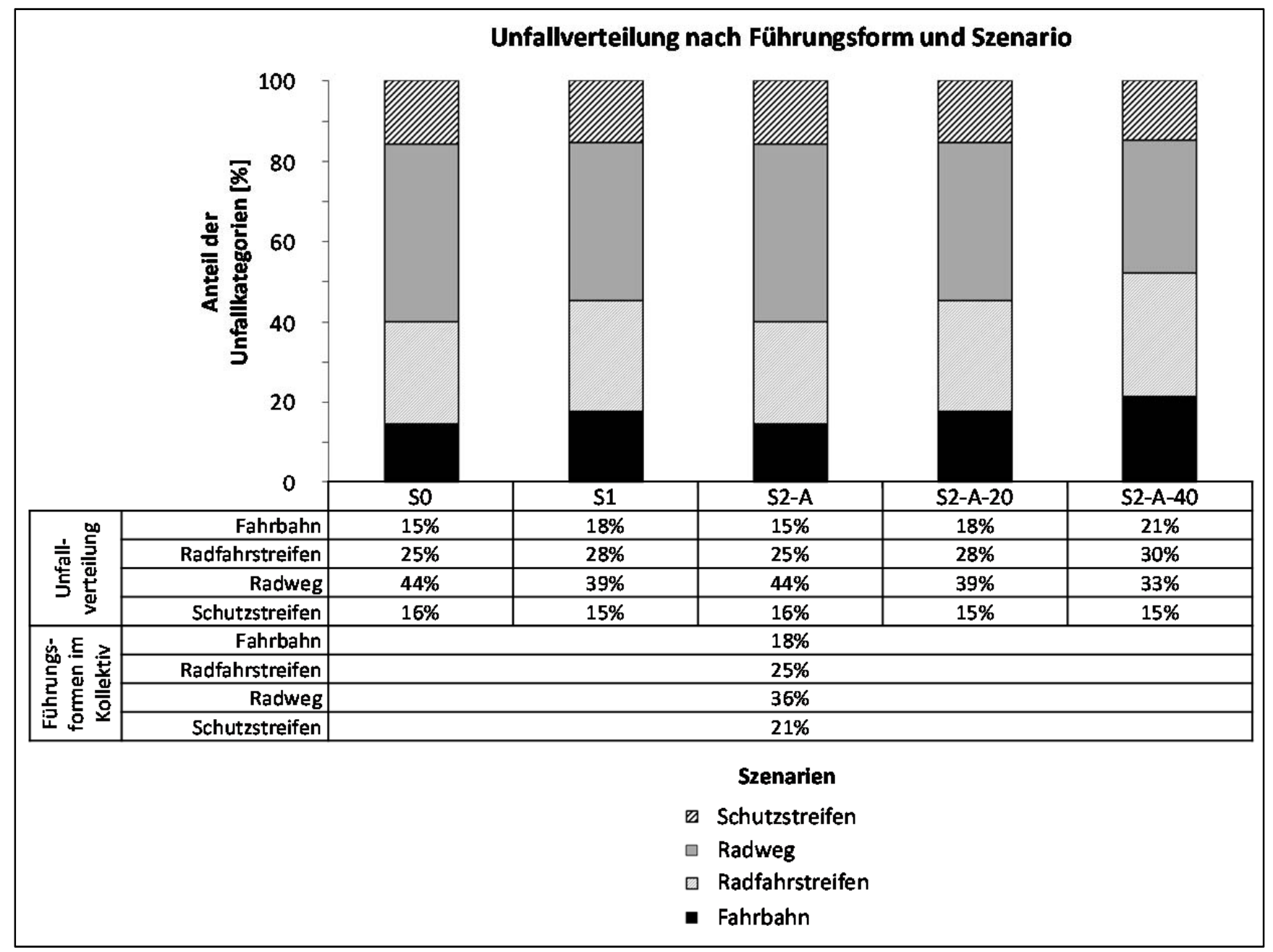

Bild 64: Ergebnisse der Szenarienberechnung zur Unfallaufteilung an Streckenabschnitten ${ }^{52}$

\section{Entwicklung der Unfalltypen}

In den makroskopischen Untersuchungen in 4.2.4, 4.3.3 und 4.4.3 hatten sich unterschiedliche Unfalltypenverteilungen gezeigt in Abhängigkeit der Einflüsse von Rad-Geschwindigkeiten, Radverkehrsstärken und des Alters der Radfahrer. Dabei wirkten sich die Einflüsse auf einzelne Unfalltypen unterschiedlich aus. 
Die Ergebnisse der Szenarienberechnung auf die Aufteilung der Unfalltypen (Tabelle 49) zeigte, dass sich unabhängig des gewählten Szenarios kaum Änderungen in der Aufteilung der Unfalltypen ergaben. Lediglich der Anteil der Unfälle im Längsverkehr nahm in fast jedem Szenario zu, während sich der Anteil der sonstigen Unfälle verringerte.

Tabelle 49: Ergebnisse der Szenarien zur Verteilung der Unfalltypen an Streckenabschnitten

\begin{tabular}{|c|c|c|c|c|c|c|c|c|c|c|c|c|c|c|c|c|}
\hline \multirow[b]{2}{*}{ Szenario } & \multirow[b]{2}{*}{$\begin{array}{l}\text { Zeit- } \\
\text { hori- } \\
\text { zont }\end{array}$} & \multicolumn{2}{|c|}{$\begin{array}{c}\text { demografi- } \\
\text { scher Wandel }\end{array}$} & \multicolumn{3}{|c|}{$\begin{array}{c}\text { Radverkehrsanteil } \\
\text { am Modal Split }\end{array}$} & \multicolumn{2}{|c|}{$\begin{array}{l}\text { Geschwin- } \\
\text { digkeit (v) }\end{array}$} & \multirow[b]{2}{*}{ 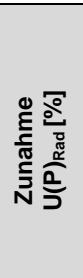 } & \multicolumn{7}{|c|}{ Anteil der Unfalltypen [\%] } \\
\hline & & 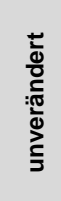 & 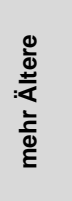 & $\begin{array}{l}\stackrel{0}{9} \\
m \\
-1\end{array}$ & ¿े & $\begin{array}{l}\circ \\
9\end{array}$ & 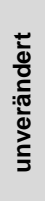 & 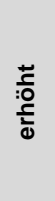 & & 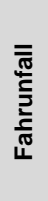 & $\begin{array}{l}\overline{\bar{\sigma}} \\
\frac{\pi}{5} \\
\dot{d} \\
\delta \\
\frac{d}{0} \\
\frac{0}{0}\end{array}$ & 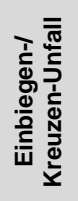 & 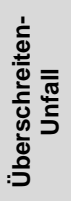 & 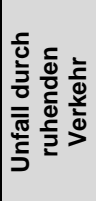 & 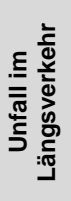 & 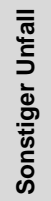 \\
\hline so & 2014 & $x$ & & $\mathrm{x}$ & & & $x$ & & \pm 0 & 4 & 10 & 15 & 4 & 15 & 27 & 25 \\
\hline S1 & 2020 & $x$ & & & $x$ & & $x$ & & 29 & 4 & 10 & 14 & 4 & 15 & 28 & 25 \\
\hline S2-A & \multirow{5}{*}{2030} & & \multirow{5}{*}{$x$} & $x$ & & & $\mathrm{x}$ & & 1 & 4 & 10 & 14 & 3 & 15 & 27 & 25 \\
\hline S2-A-20 & & & & & \multirow{2}{*}{$x$} & & $x$ & & 31 & 4 & 10 & 15 & 4 & 15 & 28 & 24 \\
\hline S2-A-20-V & & & & & & & & $x$ & 31 & 4 & 10 & 14 & 4 & 14 & 29 & 24 \\
\hline S2-A-40 & & & & & & $x$ & $x$ & & 102 & 4 & 10 & 15 & 3 & 14 & 29 & 24 \\
\hline S2-A-40-V & & & & & & $x$ & & $\mathrm{x}$ & 102 & 4 & 10 & 15 & 4 & 14 & 30 & 23 \\
\hline
\end{tabular}

Die Zunahme einzelner Unfalltypen ist in Tabelle 50 dargestellt. Unfalltypen die eine stärkere bzw. schwächere Zunahme als die Zunahme der Gesamtunfallzahl aufwiesen, wurden hervorgehoben.

Auch in der Zunahme der Unfalltypen zeigte sich, dass Unfälle im Längsverkehr in fast jedem Szenario stärker zunahmen und sonstige Unfälle schwächer zunahmen als es für die Zunahme der Gesamtunfallzahl der Radfahrer ermittelt wurde.

In Szenarien, die von einer Änderung der Altersstruktur ausgingen (S2-A - S2-A-40-V), zeigte sich ein verhältnismäßig geringer Anstieg der Unfälle durch ruhenden Verkehr.

Da sich die Effekte der Rad-Geschwindigkeiten, Radverkehrsstärken und der veränderten Altersstruktur unterschiedlich auf einzelne Unfalltypen auswirkte, konnten sich die Effekte der einzelnen Einflüsse gegenseitig aufheben. So zeigte sich bei einer alleinigen Erhöhung der Radverkehrsstärke (S1) ein geringerer Anstieg der Abbiege- und Einbiegen-/ Kreuzen-Unfälle. Durch den zusätzlichen Einfluss einer veränderten Altersstruktur und höheren Rad-Geschwindigkeiten (S2-A - S2-A-40-V) stiegen die Abbiege- und Einbiegen-/ Kreuzen-Unfälle in etwa mit dem Gesamtunfallgeschehen an.

Tabelle 50: Ergebnisse der Szenarien zur Zunahme der Unfalltypen an Streckenabschnitten

\begin{tabular}{|c|c|c|c|c|c|c|c|c|c|c|c|c|c|c|c|c|}
\hline \multirow[b]{2}{*}{ Szenario } & \multirow[b]{2}{*}{$\begin{array}{c}\text { Zeit- } \\
\text { horizont }\end{array}$} & \multicolumn{2}{|c|}{$\begin{array}{c}\text { demografi- } \\
\text { scher Wandel }\end{array}$} & \multicolumn{3}{|c|}{$\begin{array}{c}\text { Radverkehrsanteil } \\
\text { am Modal Split }\end{array}$} & \multicolumn{2}{|c|}{$\begin{array}{l}\text { Geschwin- } \\
\text { digkeit (v) }\end{array}$} & \multirow[b]{2}{*}{ 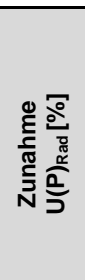 } & \multicolumn{7}{|c|}{ Zunahme der Unfalltypen [\% ] } \\
\hline & & 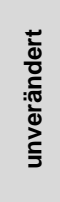 & 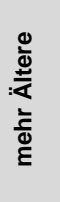 & 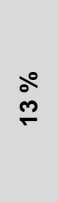 & $\begin{array}{l}\text { ลे } \\
\text { ลे }\end{array}$ & $\stackrel{9}{q}$ & 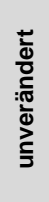 & 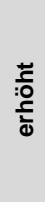 & & 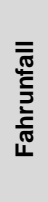 & 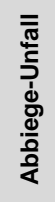 & 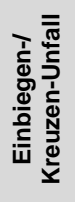 & 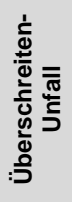 & 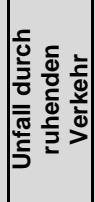 & 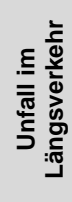 & 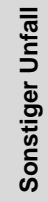 \\
\hline so & 2014 & $x$ & & $x$ & & & $x$ & & \pm 0 & 0 & 0 & 0 & 0 & 0 & 0 & 0 \\
\hline S1 & 2020 & $x$ & & & $x$ & & $\mathrm{x}$ & & 29 & 29 & 25 & 26 & 30 & 30 & 32 & 28 \\
\hline S2-A & \multirow{5}{*}{2030} & & \multirow{5}{*}{$x$} & $x$ & & & $\mathrm{x}$ & & 1 & 2 & 3 & 3 & 1 & 0 & 1 & 2 \\
\hline S2-A-20 & & & & & \multirow{2}{*}{ 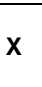 } & & $\mathrm{x}$ & & 31 & 31 & 31 & 32 & 33 & 27 & 36 & 27 \\
\hline S2-A-20-V & & & & & & & & $x$ & 31 & 32 & 32 & 31 & 35 & 25 & 39 & 24 \\
\hline S2-A-40 & & & & & & & $x$ & & 102 & 96 & 100 & 108 & 103 & 93 & 117 & 88 \\
\hline S2-A-40-V & & & & & & $X$ & & $\mathrm{x}$ & 102 & 99 & 101 & 104 & 107 & 90 & 122 & 85 \\
\hline
\end{tabular}




\subsubsection{Lichtsignalgeregelte Knotenpunkte}

\section{Unfallanzahl und Unfallschwere}

Die Ergebnisse der Szenarien ergaben sich aus den Einflüssen durch die Veränderung der Altersstruktur. Tabelle 51 zeigt die Gegenüberstellung der Ergebnisse im Hinblick auf die Veränderung von:

- Unfallanzahl

- Steigerung der Anzahl schwerer Radverkehrsunfälle (mit schwerem Personenschaden) auf Basis des erweiterten Unfallkollektivs der Makroanalyse

- Anteil der schweren Radverkehrsunfälle an allen Radverkehrsunfällen mit Personenschaden.

Für die Unfallanzahl konnte kein Anstieg bei steigenden Radverkehrsstärken nachgewiesen werden, sodass in allen Szenarien kein Anstieg ausgewiesen wurde. Durch den demografischen Wandel allerdings kann die Anzahl der Unfälle mit schwerem Personenschaden um 2 Prozentpunkte auf $16 \%$ steigen. Auch nach den vergleichenden Berechnungen auf Grundlage der Unfälle und des in die Knotenpunkte einfahrenden Radverkehrs ist ein altersbedingter Anstieg des Anteils schwerer Unfälle auf $16 \%$ zu erwarten.

Tabelle 51: Ergebnisse der Szenarienberechnung zur Unfallanzahl und -schwere an lichtsignalgeregelten Knotenpunkten

\begin{tabular}{|c|c|c|c|c|c|c|c|c|c|c|c|}
\hline \multirow[b]{2}{*}{ Szenario } & \multirow[b]{2}{*}{$\begin{array}{l}\text { Zeithori- } \\
\text { zont }\end{array}$} & \multicolumn{2}{|c|}{$\begin{array}{c}\text { demografischer } \\
\text { Wandel }\end{array}$} & \multicolumn{3}{|c|}{$\begin{array}{c}\text { Radverkehrsanteil } \\
\text { am Modal Split }\end{array}$} & \multicolumn{2}{|c|}{ Geschwindigkeit (v) } & \multirow[b]{2}{*}{$\begin{array}{l}\text { Zunahme } \\
\mathrm{U}(\mathrm{P})_{\mathrm{Rad}}[\%] 1\end{array}$} & \multirow[b]{2}{*}{$\begin{array}{c}\text { Zunahme } \\
\left.\text { U(SP) })_{\text {Rad }}[\%]\right)\end{array}$} & \multirow[b]{2}{*}{$\begin{array}{c}\text { Anteil } \\
\mathrm{U}(\mathrm{SP})_{\text {Rad }}[\%]\end{array}$} \\
\hline & & 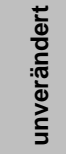 & 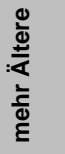 & $\stackrel{9}{m}$ & ๙̊ & ò & 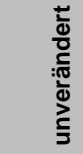 & $\begin{array}{l}\tilde{z} \\
: 0 \\
\overline{0} \\
\bar{d}\end{array}$ & & & \\
\hline So & 2014 & $x$ & & $x$ & & & $x$ & & \pm 0 & \pm 0 & 15 \\
\hline $\mathrm{S1}$ & 2020 & $x$ & & & $x$ & & $x$ & & \pm 0 & +0 & 15 \\
\hline S2-A & \multirow{5}{*}{2030} & & \multirow{5}{*}{$x$} & $x$ & & & $x$ & & \pm 0 & +2 & 16 \\
\hline S2-A-20 & & & & & \multirow{2}{*}{$x$} & & $x$ & & \pm 0 & +2 & 16 \\
\hline S2-A-20-V & & & & & & & & $x$ & \pm 0 & +2 & 16 \\
\hline S2-A-40 & & & & & & & $x$ & & \pm 0 & +2 & 16 \\
\hline S2-A-40-V & & & & & & $x$ & & $x$ & \pm 0 & +2 & 16 \\
\hline
\end{tabular}




\section{Entwicklung der Unfalltypen}

Die Radverkehrsstärken zeigten keine belastbaren Einflüsse auf die Unfalltypen, so dass aus einer Zunahme des Radverkehrs keine Veränderungen der Unfalltypen erwartet werden. Aus der altersbedingten Verteilung der Unfalltypen an lichtsignalgeregelten Knotenpunkten kann bei einem höheren Anteil Über-65-Jähriger am Radverkehr ein geringfügig steigender Anteil von Einbiegen-/ KreuzenUnfällen erwartet werden (Tabelle 52).

Tabelle 52: Ergebnisse der Szenarien zur Entwicklung der Unfalltypen an lichtsignalgeregelten Knotenpunkten

\begin{tabular}{|c|c|c|c|c|c|c|c|c|c|c|c|c|c|c|c|c|}
\hline \multirow[b]{2}{*}{ Szenario } & \multirow[b]{2}{*}{$\begin{array}{c}\text { Zeit- } \\
\text { horizont }\end{array}$} & \multicolumn{2}{|c|}{$\begin{array}{l}\text { demo- } \\
\text { grafi- } \\
\text { scher } \\
\text { Wandel }\end{array}$} & \multicolumn{3}{|c|}{$\begin{array}{l}\text { Radverkehrs- } \\
\text { anteil am } \\
\text { Modal Split }\end{array}$} & \multicolumn{2}{|c|}{$\begin{array}{l}\text { Geschwin- } \\
\text { digkeit (v) }\end{array}$} & \multirow[b]{2}{*}{ 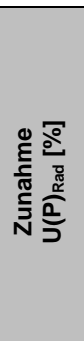 } & \multicolumn{7}{|c|}{ Zu-IAbnahme der Unfalltypen [\%] } \\
\hline & & 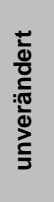 & 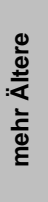 & 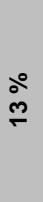 & 今 & $\begin{array}{l}8 \\
\text { i }\end{array}$ & 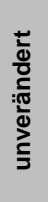 & 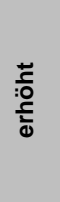 & & 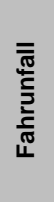 & 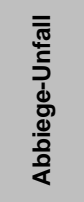 & 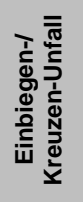 & 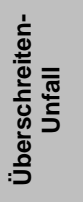 & 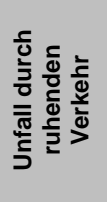 & 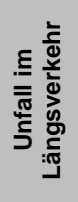 & 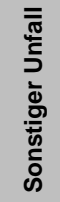 \\
\hline so & 2014 & $x$ & & $x$ & & & $x$ & & \pm 0 & 0 & 0,00 & 0,00 & 0,00 & 0,00 & 0,00 & 0,00 \\
\hline S1 & 2020 & $\mathrm{x}$ & & & $\mathrm{x}$ & & $x$ & & \pm 0 & 0 & 0,00 & 0,00 & 0,00 & 0,00 & 0,00 & 0,00 \\
\hline S2-A & \multirow{5}{*}{2030} & & \multirow{5}{*}{$x$} & $x$ & & & $x$ & & \pm 0 & 0 & $-0,12$ & 0,14 & $-0,02$ & $-0,02$ & 0,00 & 0,02 \\
\hline S2-A-20 & & & & & \multirow{2}{*}{$x$} & & $x$ & & \pm 0 & 0 & $-0,12$ & 0,14 & $-0,02$ & $-0,02$ & 0,00 & 0,02 \\
\hline S2-A-20-V & & & & & & & & $\mathrm{x}$ & \pm 0 & 0 & $-0,12$ & 0,14 & $-0,02$ & $-0,02$ & 0,00 & 0,02 \\
\hline S2-A-40 & & & & & & \multirow[b]{2}{*}{$x$} & $x$ & & \pm 0 & 0 & $-0,12$ & 0,14 & $-0,02$ & $-0,02$ & 0,00 & 0,02 \\
\hline S2-A-40-V & & & & & & & & $x$ & \pm 0 & 0 & $-0,12$ & 0,14 & $-0,02$ & $-0,02$ & 0,00 & 0,02 \\
\hline
\end{tabular}

\subsubsection{Gesamtunfallgeschehen}

Auf den Streckenabschnitten ereigneten sich etwa 3,4 Unfälle je eine Radfahrer, an den in der Mikroanalyse betrachteten Knotenpunktzufahrten etwa 2,2. Die Anteile von Unfällen mit schwerem Personenschaden an Streckenabschnitten und Knotenpunkten lagen nach den Daten der Makroanalyse in vergleichbarer Höhe (Tabelle 53).

Tabelle 53: Verteilung der heutigen Unfälle auf das Untersuchungskollektiv an Streckenabschnitten und lichtsignalgeregelten Knotenpunkten

\begin{tabular}{|l|c|c|c|c|c|c|}
\hline & Arbeitsschritt & $\begin{array}{c}\text { Anzahl Untersuchungs- } \\
\text { beispiele }\end{array}$ & $\begin{array}{c}\text { hochgerechnete } \\
\text { Radverkehrsstärke } \\
\text { (3 Jahre) }\end{array}$ & $\begin{array}{c}\text { U(P)/3 } \\
\text { Jahre }\end{array}$ & $\begin{array}{c}\text { Anteil } \\
\text { U(SP) } \\
\text { [\%] }\end{array}$ & $\begin{array}{c}\text { Unfälle je 1 Mio. } \\
\text { Radfahrer }\end{array}$ \\
\hline Streckenabschnitte & MA & 192 & 284.911 .335 & 956 & 14 & 3,36 \\
\hline \multirow{2}{*}{$\begin{array}{l}\text { lichtsignalgeregelte } \\
\text { Knotenpunkte }\end{array}$} & \multicolumn{2}{|c|}{ erweitertes Kollektiv } & & 15.520 & 15 & \\
\cline { 2 - 8 } & MA & 218 & 101.795 .671 & 223 & 13 & \\
\cline { 2 - 8 }
\end{tabular}




\section{Schlussfolgerungen}

Im Folgenden werden aus den Untersuchungsergebnissen Empfehlungen für die Radverkehrsinfrastruktur, für straßenverkehrsrechtliche Regelungen und für die Verkehrsaufklärung abgeleitet. Die Empfehlungen wurden nach verschiedenen Gruppen der Szenarien des künftigen Radverkehrs differenziert. Für die einzelnen Szenariengruppen wurden dabei Empfehlungen herausgestellt, die bei der mit den Szenarien angenommenen Entwicklung des Radverkehrs besondere Bedeutung gewinnen.

Die Empfehlungen beruhten auf den Erkenntnissen über den heutigen Radverkehr. Bereits heute bestehen Radverkehrsanlagen mit starkem Radverkehr bzw. mit höheren Geschwindigkeiten des Radverkehrs. Bereits heute sind alte Menschen im Radverkehr besonders gefährdet. Die Empfehlungen für den künftigen Radverkehr sind daher bereits heute relevant und sollten Anwendung finden, um einem Anstieg der Unfallzahlen wie in dem prognostizierten Umfang entgegenzuwirken.

\subsection{Folgerungen im Hinblick auf steigende Radverkehrsstärken (Sze- narien S1, S2-A-20, S2-A-20-V, S2-A-40, S2-A-40-V)}

\subsubsection{Streckenabschnitte mit Einmündungen und Grundstückszufahrten}

Die durch steigende Radverkehrsstärken zu erwartende Zunahme der Anzahl von Unfällen - insbesondere auch der Unfälle mit schwerem Personenschaden - erfordert einen besonderen Handlungsschwerpunkt an Streckenabschnitten.

\section{Anlagentypen}

Die Ergebnisse der Szenarienberechnung haben gezeigt, dass, wenn bei der heute üblichen Mischung teils anforderungsgerechter, teils aber auch mängelbehafteter Radverkehrsanlagen keine gezielten Sicherungsmaßnahmen an defizitären Anlagen ergriffen werden, an Verkehrsstraßen bei allen Führungsformen grundsätzlich eine Zunahme der Unfälle durch ein höheres Radverkehrsaufkommen zu erwarten ist. Verstärkte Sicherheitsdefizite ergeben sich dabei vor allem bei Radfahrstreifen und bei Mischverkehrsführungen, da die Unfallhäufigkeit dieser Anlagentypen stärker mit der Radverkehrsstärke steigen wird als bei Radwegen oder Schutzstreifen.

Grundsätzlich kommen aber in Zukunft an Verkehrsstraßen weiterhin alle bislang gebräuchlichen Führungsformen für den Radverkehr wie Radwege, Radfahrstreifen, Schutzstreifen und Mischverkehrs-Führungen in Betracht. In dem Untersuchungskollektiv zeigte sich, dass den technischen Regelwerken entsprechend ausgebildete Radverkehrsanlagen auch die in Städten mit Radverkehrsanteilen von etwa $40 \%$ auftretenden Radverkehrsstärken sicher abwickeln können.

In Straßen mit ausgeprägten Einzelhandelsnutzungen, wichtigen Zielpunkten des Radverkehrs oder einer hohen Trennwirkung durch den Kfz-Verkehr sollten allerdings insbesondere bei Radverkehrsstärken unter 3.000 Radfahrern am Tag verstärkt Führungen auf Radfahrstreifen, Schutzstreifen oder im Mischverkehr eingesetzt werden, um die Anzahl der regelwidrig linksfahrenden Radfahrer zu reduzieren. Radwege sollten unter den genannten Umständen vorrangig bei höheren Radverkehrsstärken, wie sie bei steigenden Radverkehrsanteilen am Gesamtverkehr vermehrt auftreten können, Anwendung finden, da das regelwidrige Linksfahren mit zunehmender Radverkehrsstärke abnimmt.

Wegen ihrer niedrigen Unfallzahlen und geringer Zunahme der Unfallbelastung auch bei steigenden Radverkehrsstärken (Bild 18) sollten Fahrradstraßen - soweit sich die Struktur des Straßennetzes dafür eignet, auch als Netzalternative zu Verkehrsstraßen - verstärkt Einsatz finden. 


\section{Maßnahmen zur Reduzierung eines Anstiegs der Unfallzahlen und bestimmter Unfalltypen}

Zur Reduzierung der bei steigenden Radverkehrsstärken besonders zunehmenden Längsverkehrsunfälle wird empfohlen:

- Zur Reduzierung von Unfällen mit überholenden Kfz sollten Geschwindigkeitsbegrenzungen auf 30 $\mathrm{km} / \mathrm{h}$ geprüft werden. Unfallauffällige Streckenabschnitte mit Fahrbahnführung und zwei Richtungsfahrstreifen zeigen aber auch, dass bei zwei Richtungsfahrstreifen unter den örtlichen Randbedingungen mögliche Sicherheitsgewinne durch getrennte Radverkehrsanlagen geprüft werden sollten.

- Die tatsächliche Einhaltung der bereits heute formulierten Anforderungen der technischen Regelwerke hat erhebliche Bedeutung. So erfordern Radwege zur Reduzierung von Unfällen im Längsverkehr unter Radfahrern eine Breite von über 1,6 m.

Im Bereich der straßenverkehrsrechtlichen Regelungen sollten in der VwV-StVO die Anforderungen für die Anordnung einer Benutzungspflicht differenziert nach unterschiedlichen Radverkehrsstärken angegeben werden. Das betrifft insbesondere die Angaben zur lichten Breite von Radwegen.

Insbesondere sind neben den Maßnahmen gegen zunehmende Unfälle im Längsverkehr bei Fahrbahnführungen und Radfahrstreifen Maßnahmen zur Vermeidung einer Unfallzunahme erforderlich. Unfallauffällige Radfahrstreifen zeigen z. B.,

- dass an Einmündungen weite Kurvenradien vermieden werden sollten, um Unfällen zwischen rechts einbiegenden Kfz und bevorrechtigten Radfahrern auf dem Radfahrstreifen zu begegnen, und

- dass zwischen den Radfahrstreifen und Kfz-Parkstreifen ausreichende Sicherheitsräume erforderlich sind.

\section{Maßnahmen zur Reduzierung eines Anstiegs schwerer Unfälle}

Die Makroanalyse hatte für die straßenbündigen Führungsformen des Radverkehrs (Mischverkehr, Radfahrstreifen, Schutzstreifen und Bussonderfahrstreifen) einen Zusammenhang der Radverkehrsstärke mit der Unfallkostendichte gezeigt (Bild 20). Unter den oben genannten besonderen Maßnahmen können nach der Mikroanalyse insbesondere ausreichende Sicherheitsräume zwischen Radfahrstreifen und Kfz-Parkstreifen sowie breite Bewegungsräume zwischen Straßenbahn-Bahnkörpern und dem Bord auch schweren Radverkehrsunfällen begegnen (vgl. Kap. 4.2.3 und 4.2.4).

\subsubsection{Lichtsignalgeregelte Knotenpunkte}

Für lichtsignalgeregelte Knotenpunkte sind aus höheren Radverkehrsstärken keine steigende Unfallbelastung und kein Anstieg schwerer Radverkehrsunfälle zu erwarten. Szenarienübergreifende Empfehlungen sind im Kapitel 6.4 zusammengefasst. 


\subsection{Folgerungen im Hinblick auf die Veränderung der Altersstruktur (Szenariengruppe S2-A)}

\subsubsection{Streckenabschnitte mit Einmündungen und Grundstückszufahrten}

Bei isolierter Betrachtung des demografischen Wandels (Szenario S2-A) verteilen sich die Unfälle mit der heutigen Verteilung vergleichbar auf Streckenabschnitte und lichtsignalgeregelte Knotenpunkte. Streckenabschnitte und Knotenpunkte sollten daher gleichermaßen auf die Erforderlichkeit von Sicherungsmaßnahmen hin betrachtet werden.

\section{Maßnahmen zur Reduzierung eines Anstiegs der Unfallzahlen und bestimmter Unfalltypen}

Insbesondere das von einer veränderten Altersstruktur ausgehende Szenario S2-A zeigte an Streckenabschnitten einen überproportionalen Anstieg von Abbiegen und Einbiegen-/ Kreuzen-Unfällen. Gute Sichtbeziehungen an Einmündungen und Grundstückszufahrten besitzen bei höheren Anteilen älterer Radfahrer daher herausgehobene Bedeutung. Hier besteht insbesondere bei Radwegen Handlungsbedarf.

In den Verhaltensbeobachtungen zeigte sich, dass ältere Radfahrer vermehrt regelwidrig in linker Richtung fahren. Auch wenn sich für diese Fehlverhalten keine altersspezifisch erhöhte Unfallgefährdung ermitteln ließ, gewinnen folgende Maßnahmen wegen des grundsätzlich erhöhten Unfallrisikos linksfahrender Radfahrer besondere Bedeutung:

- Über die Anforderung der derzeitigen Regelwerke hinaus sollten Radwege an Einmündungen und stark befahrenen Grundstückszufahrten künftig grundsätzlich mit aufgepflasterten Radwegüberfahrten ausgebildet werden oder geschwindigkeitsdämpfende Maßnahmen in den untergeordneten Zufahrten erfolgen.

- Im Bereich der Verkehrsaufklärung sollten Radfahrer in verstärktem Umfang für Gefahren beim regelwidrigen links Fahren an Einmündungen und stark befahrenen Grundstückszufahrten sensibilisiert werden.

- Zugleich sollten andere Verkehrsteilnehmer, insbesondere Kfz-Führer, deutlich verstärkt dafür sensibilisiert werden, an Einmündungen und Grundstückszufahrten in beide Richtungen auf die Radverkehrsanlagen und den Gehweg zu blicken.

- Im Bereich des Straßenverkehrsrechts sollten die Bußgelder für nicht zulässiges Linksfahren im Hinblick auf den Gefährdungstatbestand angehoben werden.

- In den Verhaltensbeobachtungen zeigte sich zugleich, dass ältere Radfahrer in regelwidrig linker Richtung vermehrt den Gehweg nutzen. Eine Höhentrennung zwischen Geh- und Radwegen kann das regelwidrige linke Radfahren auf Gehwegen reduzieren. Eine derartige Höhentrennung kann aber nur eingesetzt werden, wenn zum Überholen ausreichende Radwegbreiten vorhanden sind. Die visuelle Erkennbarkeit und die Tastbarkeit sind zu berücksichtigen.

\section{Maßnahmen zur Reduzierung schwerer Unfälle}

Fast alle in der Untersuchung betrachteten Unfälle Über-65-Jähriger mit schwerem Personenschaden ereigneten sich bei Nutzung von Radwegen oder Radfahrstreifen als Einbiegen-/ Kreuzen-Unfälle. Die oben empfohlenen Maßnahmen an Einmündungen und stark befahrenen Grundstückszufahrten können daher auch schweren Unfällen begegnen. 


\subsubsection{Lichtsignalgeregelte Knotenpunkte}

An lichtsignalgergelten Knotenpunkten ist vor dem Hintergrund einer veränderten Altersstruktur ein leichter Anstieg von Einbiegen-/ Kreuzen-Unfällen zu erwarten. Da sich diese Unfälle zumeist durch Rotlichtmissachtungen begründen, sollten an unfallauffälligen Knotenpunkten mögliche Einflüsse aus den Signalschaltungen und der Erkennbarkeit der Signalgeber geprüft werden. Weiterhin sollten Signalabschaltungen in den Nachtzeiträumen überprüft werden.

Auch bei einer Zunahme der Einbiegen-/ Kreuzen-Unfälle verbleibt ein hoher Anteil von Abbiegeunfällen. An lichtsignalgeregelten Knoten müssen daher verstärkt signaltechnische Sicherungen mit konfliktfreien Schaltungen eingesetzt werden.

Insbesondere unter den Annahmen des Szenarios S2-A, also mehr Über-65-Jährigen mit Fahrrädern ohne elektrische Unterstützung, besteht Forschungsbedarf, inwieweit die in den Richtlinien für Lichtsignalanlagen angesetzten Räumgeschwindigkeiten von Radfahrern einem künftig höheren Anteil älterer Radfahrer mit niedrigeren Geschwindigkeiten angemessen sind.

\section{Maßnahmen zur Reduzierung schwerer Unfälle}

Nach den Daten der Mikroanalyse ereigneten sich die meisten schweren Unfälle Über-65-Jähriger bei Radweg- bzw. Radfahrstreifenführungen mit einbiegenden/ kreuzenden bzw. abbiegenden Kfz. Dies unterstreicht, dass bei Radweg- und Radfahrstreifenführungen verstärkt signaltechnische Sicherungen mit konfliktfreien Schaltungen eingesetzt werden müssen. 


\subsection{Folgerungen im Hinblick auf steigende Radverkehrs- Geschwindigkeiten (Szenarien S2-A-20-V, S2-A-40-V)}

\subsubsection{Streckenabschnitte mit Einmündungen und Grundstückszufahrten}

Die in Zusammenhang mit steigenden Radverkehrsstärken bei höheren Geschwindigkeiten zu erwartende Zunahme der Anzahl von Unfällen - insbesondere auch der Unfälle mit schwerem Personenschaden - erfordert einen besonderen Handlungsschwerpunkt an Streckenabschnitten.

\section{Maßnahmen zur Reduzierung eines Anstiegs der Unfallzahlen und bestimmter Unfalltypen}

Die Szenarienberechnungen für eine erhöhte Geschwindigkeit des Radverkehrs zeigten eine ähnliche Entwicklung der Unfallzahlen wie bei steigenden Radverkehrsstärken und unveränderten Geschwindigkeiten. Zur Eignung der unterschiedlichen Anlagentypen und den erforderlichen Sicherungsmaßnahmen können daher grundsätzlich die für steigende Radverkehrsstärken genannten Empfehlungen gelten.

Im Vergleich mit unveränderten Geschwindigkeiten kann allerdings eine etwas stärkere Zunahme von Längsverkehrs-Unfällen erwartet werden. Dies unterstreicht zum einen die Notwendigkeit ausreichender Breiten von Radwegen und Radfahrstreifen. In der Mikroanalyse zeigten sich darüber hinaus auffällige Überholunfällen mit Kfz in Straßen mit zwei Richtungsfahrstreifen und höheren Radfahrergeschwindigkeiten. Bei Fahrbahnführungen sollte daher verstärkt geprüft werden, inwieweit Begrenzungen der Kfz-Geschwindigkeit auf $30 \mathrm{~km} / \mathrm{h}$ die Sicherheit des Radverkehrs erhöhen können. Bei zwei Richtungsfahrstreifen sollte auch geprüft werden, inwieweit getrennte Radverkehrsführungen unter den örtlichen Randbedingungen zu einer höheren Radverkehrssicherheit beitragen können.

Die Makro- und die Mikroanalyse hatten darüber hinaus bei höheren Radverkehrsgeschwindigkeiten höhere Anteile von Unfällen durch ruhenden Verkehr aufgezeigt, bei denen gerade Straßen mit Mischverkehrs- oder mit Radfahrstreifen-Führungen auffällig waren. Sind bei RadfahrstreifenFührungen schon allein zur Begrenzung der Unfallzuwächse aus höheren Radverkehrsstärken besonders ausreichende Sicherheitsräume zu Parkstreifen erforderlich, sollten bei höheren Geschwindigkeiten auch bei Mischverkehrsführungen markierte oder bauliche Sicherheitsräume zwischen der Fahrbahn und Parkstreifen geprüft werden.

Aus ersten Untersuchungen über Unfälle von Pedelec-Fahrern geht hervor, dass eine Unterschätzung der Geschwindigkeiten von Elektrofahrrädern durch andere Verkehrsteilnehmer in Zusammenhang mit einem Unfallschwerpunkt an Einmündungen und Kreuzungen unfallbeeinflussend sein kann. Pedelec-Nutzer sollten daher verstärkt auf besondere geschwindigkeitsbedingte Risiken, wie sie etwa an Einmündungen oder Grundstückszufahrten und Knotenpunkten auftreten, sensibilisiert werden. Potenzielle Konfliktgegner wie insbesondere Kfz-Führer sollten für die Gefahren aus höheren Geschwindigkeiten von Radfahrern sensibilisiert werden.

Die polizeilichen Unfallaufnahmen differenzieren künftig auch nach einem Fahrzeugtyp „Pedelec“53 und „E-Bike“54. Hier sollte künftig geprüft werden, ob vermehrte Unfälle zwischen Pedelec-Nutzern

53 Fahrrad mit Trethilfe und einem elektromotorischen Hilfsantrieb mit einer maximalen Nenndauerleistung von 0,25 kW, dessen Unterstützung sich mit zunehmender Fahrgeschwindigkeit progressiv verringert und spätestens beim Erreichen von $25 \mathrm{~km} / \mathrm{h}$ unterbrochen wird 
und Fußgängern einer Freigabe von Fußgängerflächen für den Radverkehr (Freizeitwege, Fußgängerzonen) und dem Einsatz von Radwegen in Straßen mit hohem Fußgängeraufkommen bei zunehmender Pedelec-Nutzung und hierdurch steigenden Radfahrergeschwindigkeiten entgegenstehen.

\section{Maßnahmen zur Reduzierung schwerer Unfälle}

Die zuvor genannten Maßnahmen sind sowohl dazu geeignet geschwindigkeitsbedingte Unfälle als auch Unfälle mit schwerem Personenschaden zu reduzieren. Darüber hinausgehende spezielle Maßnahmen zur Reduzierung der Unfallschwere lassen sich aus den Untersuchungsergebnissen nicht ableiten.

\subsubsection{Lichtsignalgeregelte Knotenpunkte}

\section{Maßnahmen zur Reduzierung eines Anstiegs der Unfallzahlen und bestimmter Unfalltypen}

Eine durch die Radfahrergeschwindigkeiten systematisch beeinflusste Zunahme von Unfällen an lichtsignalgeregelten Knotenpunkten konnte für die Szenarien nicht ermittelt werden. In der Mikroanalyse zeigten sich mehrere sonstige Fahrunfälle mit der Unfallursache 13 (Geschwindigkeit in anderen Fällen) an lichtsignalgeregelten Knotenpunkten. Radfahrergruppen, die - wie insbesondere jüngere Erwachsene oder Pedelec-Nutzer - typischerweise mit höheren Geschwindigkeiten fahren, sollten daher verstärkt für besondere geschwindigkeitsbedingte Risiken sensibilisiert werden.

\section{Maßnahmen zur Reduzierung schwerer Unfälle}

Mit dem Ziel einer Reduzierung schwerer Unfälle mit ausgeprägten Kopfverletzungen sollte weiterhin für die Helmnutzung geworben werden. 


\subsection{Ergänzende szenarienübergreifende Folgerungen}

Für die Streckenabschnitte von Verkehrsstraßen kommen auch bei höheren Radverkehrsstärken, mehr älteren Radfahrern und höheren Radverkehrsgeschwindigkeiten grundsätzlich Radwege, Radfahrstreifen, Schutzstreifen und Fahrbahnführungen in Betracht. Erforderlich sind allerdings besondere, für die Szenarien genannte Sicherungsmaßnahmen.

An lichtsignalgeregelten Knotenpunkten sprechen die im Vergleich zur Führung auf Geh- und Radwegen niedrigeren Unfallraten für den vermehrten Einsatz von Radfahrstreifen- oder Fahrbahnführungen. Diese sichern den Radverkehr auch bei zunehmenden Radverkehrsstärken.

An untergeordneten Knotenarmen mit Radfahr- bzw. Schutzstreifen oder Fahrbahnführung des Radverkehrs sollten verstärkt aufgeweitete Aufstellflächen eingesetzt werden. Bei der Flächenbemessung sollte der zunehmende Radverkehr beachtet werden. 


\section{Anhang}

\subsection{Theorie der Verallgemeinerten linearen Modelle}

Beim im Projekt verwendeten Modell handelt es sich um ein verallgemeinertes lineares Modell. In verallgemeinert linearen Modellen werden Einflussfaktoren über Exponential- oder Potenzfunktionen berücksichtigt und multiplikativ miteinander verknüpft. Dies ist beispielhaft in Formel 4 dargestellt:

Formel 4:

$U=a \cdot q^{b_{1}} \cdot L^{b_{2}} \cdot e^{\sum_{i=3}^{n} X_{i} \cdot b_{i}}$

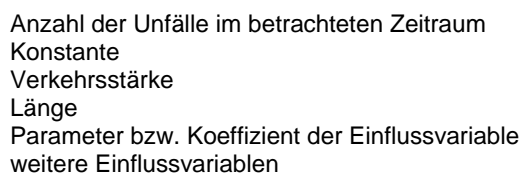

Die über Potenzfunktionen dargestellten Einflüsse der Streckenlänge und der Radverkehrsstärke beschreiben im Modell Expositionsgrößen. Sie haben die Eigenschaft, dass bei ihrem Nichtauftreten $($ Wert $=0)$ die Unfallanzahl den Wert Null annimmt. Der steigende Einfluss von der Radverkehrsstärke oder der Streckenlänge führt zu einem steigenden Effekt auf die Unfallanzahl (z. B. progressiv, linear oder degressiv).

Die über Exponentialfunktionen beschrieben Einflussgrößen (z. B. Anzahl der Anschlussknotenpunkte) sind Risikogrößen im Modell. Die exponentielle Darstellung der Risikogrößen und die multiplikative Verknüpfung der einzelnen Größen im Modell führen dazu, dass nur von Null abweichende Werte der Risikogrößen Auswirkungen auf die Unfallanzahl haben. Diese Risikogrößen können als dichotome (z. B. Umfeldnutzung), kategoriale (z. B. Radverkehrsanlage) oder stetige Funktionen (z. B. bebaute Fläche) in das Modell aufgenommen werden.

\subsubsection{Modellaufbau}

Der Aufbau des Modells erfolgte schrittweise. Das Modell ohne Einflussfaktoren (Nullmodell) beschreibt den Mittelwert der Unfälle des Untersuchungskollektivs. Anschließend wurde jede einzelne zur Verfügung stehende Einflussgröße einzeln in das Modell aufgenommen und auf seinen Erklärungsanteil der systematischen Streuung überprüft. Die systematische Streuung beschreibt dabei die unterschiedliche Charakteristik der einzelnen Untersuchungspunkte (Strecken) im Modell. Die Einflussgröße mit dem höchsten Erklärungsanteil wurde in das Modell aufgenommen. Im weiteren Verlauf wurden erneut alle Einflussgrößen in das erweiterte Modell auf ihre Varianzerklärung geprüft. Sofern eine Korrelation mit den bereits beinhalteten Größen im Modell ausreichend ausgeschlossen werden konnte und die Einflussgröße einen signifikanten Erklärungsanteil zur Streuung beigetragen hatte, wurde die Größe mit der höchsten Varianzerklärung in das Modell aufgenommen.

\subsubsection{Modellprüfung}

Nach der Aufnahme aller signifikanten Einflussgrößen, wurde das Modell geprüft auf:

- die Anpassung an die Stichprobe und,

- ob die enthaltenen Aussagen verallgemeinert werden können.

Hierbei kamen Residuen und Wahrscheinlichkeitsplots zu Anwendung.

Die Wahrscheinlichkeitsplots überprüften die Annahme, dass die standardisierten Residuen im Modell einer Normalverteilung folgten. 
Hierzu wurden die empirischen Quantile der standardisierten Residuen den theoretischen Quantilen der Normalverteilung in einem "Q-Q-Plot“ gegenübergestellt. Bei annähernder Normalverteilung der standardisierten Residuen ergibt sich die Gerade „y=x“. Ähnlich wurden auch die Summenganglinien der Wahrscheinlichkeiten der empirischen standardisierten Residuen den theoretischen Werten der Normalverteilung in einem „P-P-Plot“ gegenübergestellt (vgl. Bild 66).

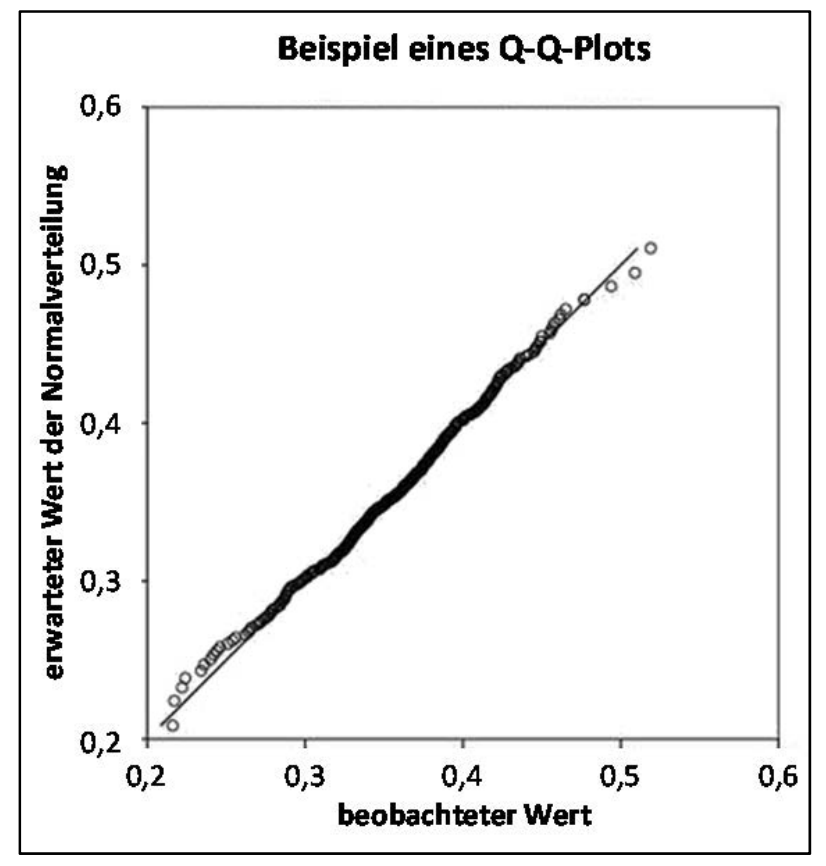

Bild 65: Beispiel eines Q-Q-Plots

Die erstellten Residuenplots dienten dazu, die Abweichung zwischen den empirischen und den modellierten Unfallzahlen gegenüberzustellen. An die Residuen wurden dabei folgende Bedingungen gestellt:

- Homoskedastizität

- Keine Linearität

Im Anschluss wurde daher die unsystematische Schwankung der Residuen um den Mittelwert „0“ (Homoskedastizität) bezüglich der Streckenlänge und der Radverkehrsstärke untersucht. Eine Linearität läge vor, wenn eine systematische Anordnung der Residuen bezüglich der betrachteten Einflussgröße erkennbar wäre. Ein idealisierter Residuenplot ist in Bild 66 dargestellt. 


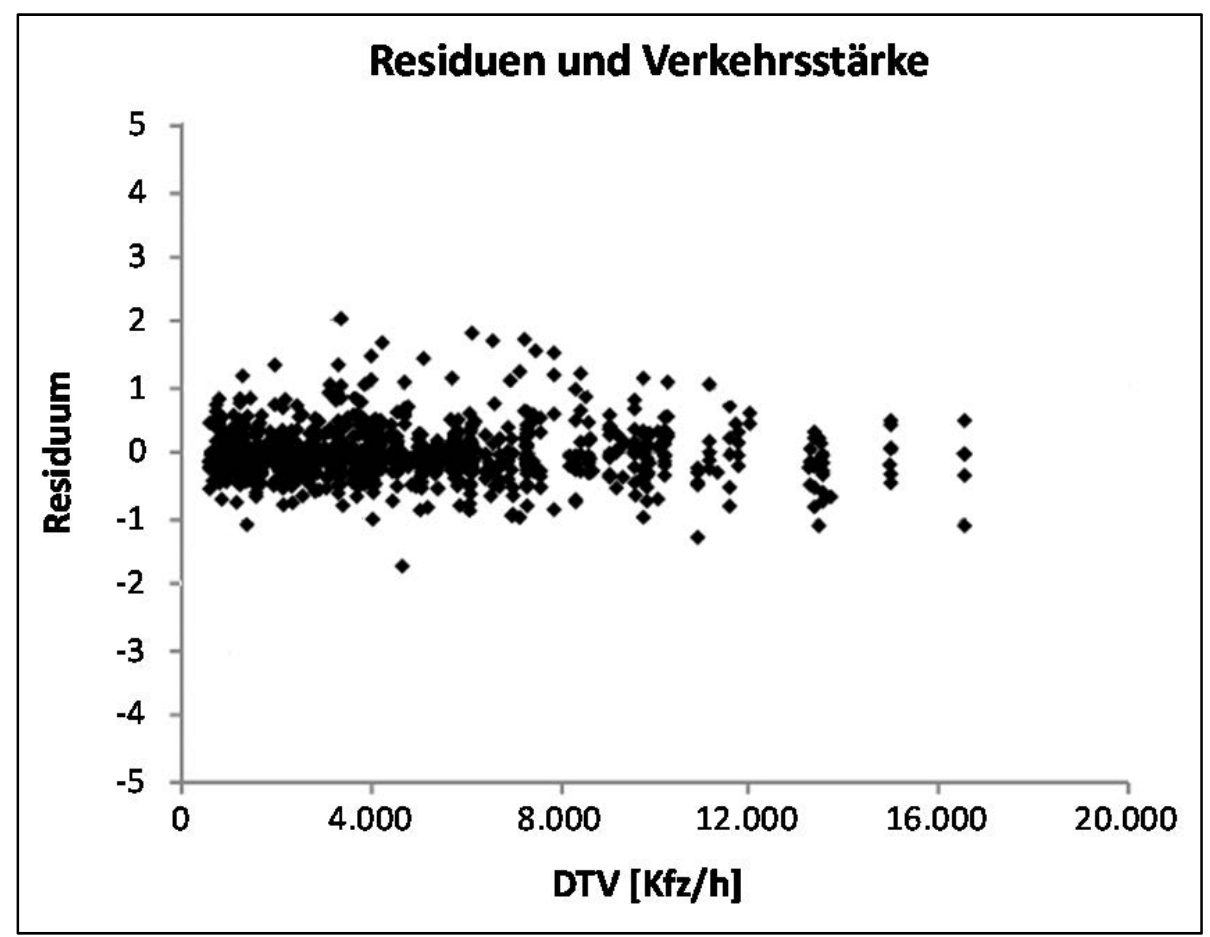

Bild 66: Beispiel eines Residuenplots über die Einflussgröße DTV

Im nächsten Schritt wurde die systematische Varianz in Cure-Plots geprüft. Cure-Plots stellen den kumulierten Verlauf der Residuen bezüglich einer Einflussgröße (Streckenlänge oder Radverkehrsstärke) dar. Die Darstellung kumulierter Residuen ermöglichte es Abschnitte der Einflussgröße zu verdeutlichen, in denen die Residuen systematisch von „0“ abweichen, bzw. in denen die Unfallanzahl systematisch über- oder unterschätzt wurde.

Als Vertrauensbereich zur Prüfung der systematischen Varianz entwickelten HAUER \& BAMFO (1997) die Grenzen $\pm 2 \delta^{\star}$ in denen sich die kumulierten Residuen bei Fehlen von systematischer Varianz bewegen sollten. Systematische Varianz kann auftreten, wenn vereinzelte Bereiche der Haupteinflussgröße in den Daten über- oder unterproportional häufig auftreten. 
Das Beispiel in Bild 67 stellt einen solchen Cure-Plot dar. In dem im Bereich von etwa 10.000-25.000 Einwohnern zeigt sich, dass eine systematische Abweichung der Residuen zu erkennen ist. Diese folgen aus dem überproportionalen Auftreten eines Bereichs der Haupteinflussgröße, weshalb das Modell stärker an diesen Bereich angepasst wird und dafür andere Bereiche schlechter angepasst werden.

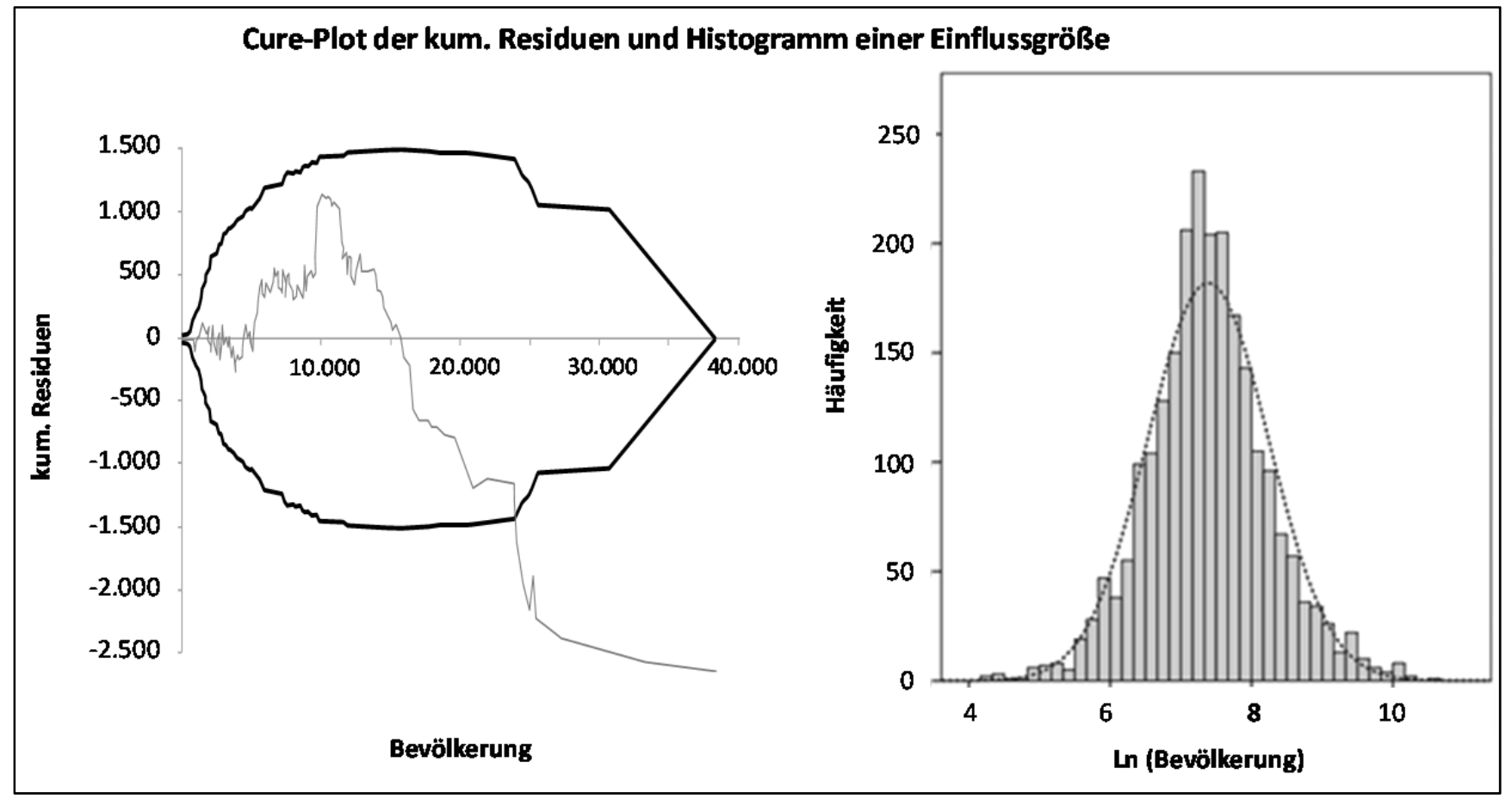

Bild 67: Beispiel Cure-Plot über die Einwohneranzahl und Histogramm der logarithmierten Einwohnerzahl

Des Weiteren musste das Modell auf Ausreißer hin überprüft werden. Hierzu wurden die Distanzmaße Hebelwert und Cook-Distanz verwendet. Die Cook-Distanz beschreibt den Einfluss, den der Ausschluss eines Falls auf die Residuen im Modell bewirkt. Als Grenzwert wurde von Hutcheson et al. (1999) der Wert nach Formel 5 angegeben.

Formel 5:

$C d=\frac{4}{(n-k)}$

Cd Cook-Distanz

k Anzahl der Freiheitsgrade

n Kollektivgröße (Betrachtungsfälle)

Der Hebelwert dient der Beschreibung der Hebelwirkung eines Falls auf das Regressionsmodell. Hohe Hebelwerte wirken sich demnach stark auf die Koeffizienten der Modellparameter aus. Als Grenzwert für den Hebelwert gilt:

Formel 6:

$H w=\frac{3 \cdot(k+1)}{n}$

Hw Hebelwert

K Anzahl der Freiheitsgrade

n Kollektivgröße (Betrachtungsfälle)

Das Überschreiten der Grenzwerte für die Distanzmaße führte nicht zu einem automatischen Ausschluss des Falls im Modell, bedarf aber einer manuellen Prüfung der inhaltlichen Gründe bzw. Besonderheiten des Falls. 


\subsubsection{Modellinterpretation}

Anhand eines Beispiels (Bild 68) soll die Funktionsweise und Interpretation der Modelle zur Beschreibung des Unfallgeschehens erläutert werden. Als Einflussgrößen auf die Unfallanzahl im nachfolgenden Beispiel beinhaltet das Modell die Bevölkerung (BEV), die Erwerbstätigen (EWT), und die Knotenpunktdichte im untergeordneten Straßennetz.

\begin{tabular}{|c|c|c|c|c|c|c|c|c|}
\hline \multirow{2}{*}{$\begin{array}{l}\text { Modell } \\
U(P)\end{array}$} & \multirow[b]{2}{*}{ Parameter } & \multirow[b]{2}{*}{ Koeffizienten } & \multirow[b]{2}{*}{$\mathrm{p}$} & \multicolumn{2}{|c|}{ neg. Binomialverteilung } & \multicolumn{3}{|c|}{ Poissonverteilung } \\
\hline & & & & $\begin{array}{l}\text { Verteilungs- } \\
\text { parameter }\end{array}$ & $\begin{array}{c}\text { Pearson- } \\
\text { Abweichung }\end{array}$ & $\begin{array}{c}\text { Pearson- } \\
\text { Abweichung }\end{array}$ & $d f$ & $\begin{array}{l}\text { Anpassungs } \\
\text { güte } \\
\end{array}$ \\
\hline \multicolumn{2}{|c|}{ Nullmodellk } & $-3,381 * * * *$ & ,000 & 6,310 & 2341,223 & 553385,433 & 2342 & 236,288 \\
\hline \multicolumn{2}{|c|}{ Exposition $\mathrm{k}$} & $-14,889 * * * *$ & , 000 & 0,243 & 2336,907 & 15903,088 & 2336 & 6,808 \\
\hline & LN_BEV & $1,366 * * * *$ & ,000 & & & & & \\
\hline & LN_EWT &, $316 * * * *$ & ,000 & & & & & \\
\hline \multicolumn{2}{|c|}{ Endmodellk } & $-16,152 * * * *$ & ,000 & 0,21 & 2324,53 & 14424,185 & 2324 & 6,207 \\
\hline & LN_BEV & $1,326 * * * *$ & ,000 & & & & & \\
\hline & LN_EWT &, $263 * * * *$ & ,000 & & & & & \\
\hline & unter_KP_DICHTE &, $049 * * * *$ & ,000 & & & & & \\
\hline
\end{tabular}

Bild 68: Beispielparametersatz

Im ersten Modellschritt wurden keine Einflussparameter im Modell berücksichtigt. Das sogenannte Nullmodell gibt den Erwartungswert für das Untersuchungskollektiv wieder. An dem Nullmodell wurde die Verbesserung weiterer Modelle bei Hinzunahme weiterer Einflussgrößen getestet.

Um die Verbesserung der Modelleffekte darzustellen, wurden, neben der Anpassung an eine negative Binomialverteilung, die Modelle auf Basis der Poissonverteilung konstruiert. Bei einer perfekten Anpassung an eine Poissonverteilung entspricht die Pearson-Abweichung der Anzahl der Freiheitsgrade und die Anpassungsgüte nimmt demnach den Wert 1 an. Die Abweichung der Anpassungsgüte von 1 ist ein Maß für die verbleibende systematische Streuung im Modell. Die Reduzierung der Abweichungen beim Aufbau des Modells wurde daher als Indikator genutzt für die Verbesserung der Modellgüte (Anpassungsgüte). Ziel war es die Anpassungsgüte auf 1 zu reduzieren. Dann liegt eine perfekte Anpassung an eine Poissonverteilung vor. Die Reduzierung des Werts der Anpassungsgüte vom Nullmodell über das Modell mit Expositionsgrößen bis hin zum Endmodell verdeutlicht die Erhöhung des Anteils der erklärten Streuung an der Gesamtstreuung. Die Verbesserung der Anpassungsgüte im Beispiel von 236,288 im Nullmodell auf 6,207 im Endmodell erklärt demnach $97 \%$ der systematischen Streuung:

Formel 7:

$\frac{(236,288-1)}{(6,207-1)}=97 \%$

Das Endmodell kann dann in der folgenden Form dargestellt werden:

Formel 8:

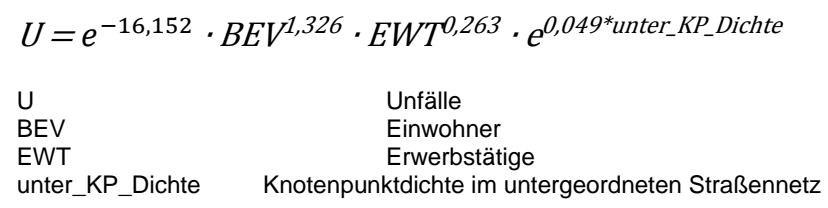




\subsubsection{Modellparameter}

In der folgenden Tabelle 54 sind die im Unfallmodell verwendeten Parameter aufgelistet:

Tabelle 54: Untersuchte Modellparameter

\begin{tabular}{|c|c|}
\hline \multicolumn{2}{|l|}{ Streckenparameter } \\
\hline Parameter & Einheit/ Ausprägungen \\
\hline Streckenlänge & {$[\mathrm{m}]$} \\
\hline Radverkehrsführung & $\begin{array}{l}\text { Fahrbahn (Mischverkehr), Radweg, gem. Geh-/Radweg, Gehweg/Radfahrer frei, selbständige Wegeverbindung, } \\
\text { Radfahrstreifen, Schutzstreifen, nicht benutzungspfl. Radweg, Berliner Lösung Schutzstreifen, 2-Rtg.-Radweg, } \\
\text { Busstreifen- RV freigegeben, Fahrradstraße }\end{array}$ \\
\hline Anzahl Anschlussknotenpunkte & - \\
\hline Art des Belags & Asphalt, Beton ohne Fase, Beton mit Fase, sonstige \\
\hline Breite Radverkehrsanlage & {$[\mathrm{m}]$} \\
\hline Befahrbarkeit des Belags & gut, mittel, schlecht \\
\hline Radweg niveaugleich durchgeführt & Niveaugleich, höhenungleich ( $\geq 0,02 \mathrm{~m}$ ) \\
\hline Furtabsetzung & {$[\mathrm{m}]$} \\
\hline \multicolumn{2}{|l|}{ Parameter des Radverkehrs } \\
\hline Parameter & Einheit/ Ausprägungen \\
\hline Rad-DTV & Durchschnittlich täglicher Radverkehr [Rad/24h] \\
\hline Radverkehrsanteil & - \\
\hline mittlere Radgeschwindigkeit $\mathbf{v}_{m, R a d}$ & {$[\mathrm{~km} / \mathrm{h}]$} \\
\hline $\mathbf{V}_{15, \mathrm{Rad}}$ & {$[\mathrm{km} / \mathrm{h}]$} \\
\hline $\mathbf{V}_{50, \text { Rad }}$ & {$[\mathrm{km} / \mathrm{h}]$} \\
\hline $\mathrm{V}_{85, \mathrm{Rad}}$ & {$[\mathrm{km} / \mathrm{h}]$} \\
\hline \multicolumn{2}{|l|}{ Parameter des Verkehrs } \\
\hline Parameter & Einheit/ Ausprägungen \\
\hline DTV & Durchschnittlich täglicher Verkehr [Kfz/ 24h] \\
\hline Städtebauliche Nutzung & $\begin{array}{l}\text { Wohnnutzung, Mischnutzung, Geschäfte, Parkanlage/Waldgebiet, Verwaltung, Gewerbegebiet, Verwal- } \\
\text { tung/Wohnen, Stadion, Schloss/öffentl. Gebäude, Bahnhof/Schienenanlagen, Friedhof, Berliner Kindl Braue- } \\
\text { rei/Großparkplatz/Wohnen, Klinikum, andere }\end{array}$ \\
\hline Geschwindigkeits-beschränkung & $50 \mathrm{~km} / \mathrm{h}, 30$ km/h, sonstiges, i. d. R. 50 km/h (Teilabschnitte oder zeitl. Befristet 30 km/h) \\
\hline Schwerverkehrsanteil & - \\
\hline Anzahl Grundstückszufahrten & - \\
\hline Parkaufstellung & längs, schräg, quer, absolutes Halteverbot, eingeschränktes Halteverbot, baulich nicht mgl., kein Parken \\
\hline Einbahnstraße & ja, nein \\
\hline Straßenraumbreite & {$[\mathrm{m}]$} \\
\hline $\begin{array}{l}\text { Entwurfssituationen der RASt } 06 \\
\text { (FGSV, 2006) }\end{array}$ & $\begin{array}{l}\text { Wohnweg, Wohnstraße, Sammelstraße, Quartierstraße, dörfliche Hauptstraße, örtliche Einfahrtstraße, örtliche } \\
\text { Geschäftsstraße, Hauptgeschäftsstraße, Gewerbestraße, Industriestraße, Verbindungsstraße, anbaufreie Straße, } \\
\text { keine Festlegung }\end{array}$ \\
\hline Öffentlicher Personennahverkehr & keiner, Bus, Straßenbahn mit eigenem Bahnkörper, Straßenbahn im Fahrbahnquerschnitt \\
\hline
\end{tabular}




\subsection{Unfallkostensätze}

Die Berechnung angepasster Unfallkostensätze diente dazu Unterschiede in der Verunglücktenstruktur der Radfahrer bei Unfällen mit Personenschaden Rechnung zu tragen. Auf Basis der Verletzungsschwere der Verunglückten, der Unfallkategorie und den pauschalen Unfallkostensätzen ergaben sich die (indirekt) angepassten Kostensätze wie folgt:

Formel 9:

$W U a(S P)=\frac{\left(\begin{array}{c}V(G T) * W V(G T)+V(S V) * W V(S V)+V(L V)^{55} * W V(L V)+ \\ U(G T) * W U S(G T)+U(S V) * W U S(S V)\end{array}\right)}{U(S P)}$

Formel 10:

$W U a(L V)=\frac{(V(L V) * W V(L V)+U(L V) * W U S(L V))}{U(L V)}$

Formel 11:

$W U a(P)=\frac{\left(\begin{array}{c}V(G T) * W V(G T)+V(S V) * W V(S V)+V(L V) * W V(L V)+ \\ U(G T) * W U S(G T)+U(S V) * W U S(S V)+U(L V) * W U S(L V)\end{array}\right)}{U(P)}$

mit: $\quad$ WUa(SP) $\quad$ angepasster Unfallkostensatz der Unfälle mit Schwerverletzten oder Getöteten

Preisstand 2009 (BAST, 2011):

WUa(LV) angepasster Unfallkostensatz der Unfälle mit leichtem Personenschaden

WUa(P) angepasster Unfallkostensatz der Unfälle mit Personenschaden

$\mathrm{V}(\mathrm{GT}) \quad$ Getötete

$\mathrm{V}(\mathrm{SV}) \quad$ Schwerverletzte

$\mathrm{V}(\mathrm{LV}) \quad$ Leichtverletzte

WV (GT) Kostensatz für Getötete $\quad 996.412 € / G T$

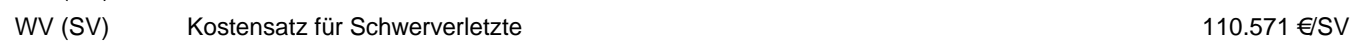

WV(LV) Kostensatz für Leichtverletzte $\quad 4.416 € / L V$

WUS(GT) Kostensätze für Sachschaden bei Unfällen mit Getöteten $\quad 40.108 € / U(G T)$

WUS(SV) Kostensätze für Sachschaden bei Unfällen mit Schwerverletzten $\quad 19.215 € / U(S V)$

WUS(LV) Kostensätze für Sachschaden bei Unfällen mit Leichtverletzten $\quad 13.036 € / U(L V)$

U(SP) Unfälle mit Schwerverletzten oder Getöteten

$\mathrm{U}(\mathrm{LV}) \quad$ Unfälle mit Leichtverletzten

$U(P) \quad$ Unfälle mit Personenschaden

Unter Verwendung der Daten des Unfallkollektivs ergaben sich die folgenden angepassten Unfallkostensätze:

${ }^{55}$ Leichtverletzte, welche bei Unfällen mit schwerem Personenschaden verunglückten 
Formel 12:

$W U a(S P)=\frac{\left(\begin{array}{c}16 G T * 996.412 \frac{€}{G T}+473 S V * 110.571 \frac{€}{S V}+57 L V * 4.415 \frac{€}{L V}+ \\ 16 U(G T) * 40.108 \frac{€}{U(G T)}+450 U(S V) * 19.215 \frac{€}{U(S V)}\end{array}\right)}{466 U(S P)}$

$=166.915 €$

Formel 13:

$W U a(L V)=\frac{\left(3.291 L V * 4.415 \frac{€}{L V}+2.815 U(L V) * 13.036 \frac{€}{U(L V)}\right)}{2.815 U(L V)}$

$=18.198 €$

Formel 14:

$W U a(P)=\frac{\left(\begin{array}{c}16 G T * 996.412 \frac{€}{G T}+473 S V * 110.571 \frac{€}{S V}+3.348 L V * 4.415 \frac{€}{L V}+ \\ 16 U(G T) * 40.108 \frac{€}{U(G T)}+450 U(S V) * 19.215 \frac{€}{U(S V)}+2.815 L V * 13.036 \frac{€}{U(L V)}\end{array}\right)}{3.281 U(S P)}$

$=39.320 €$

Tabelle 55: angepasste Unfallkostensätze der Radfahrunfälle (nach Preisstand 2009)

\begin{tabular}{|l|c|c|c|}
\hline \multicolumn{3}{|c|}{ Unfallkostensätze } \\
\hline angepasst & WUa(P) & WUa(SP) & WUa(LV) \\
\hline
\end{tabular}


7.3 Unfallverteilung nach Führungsform

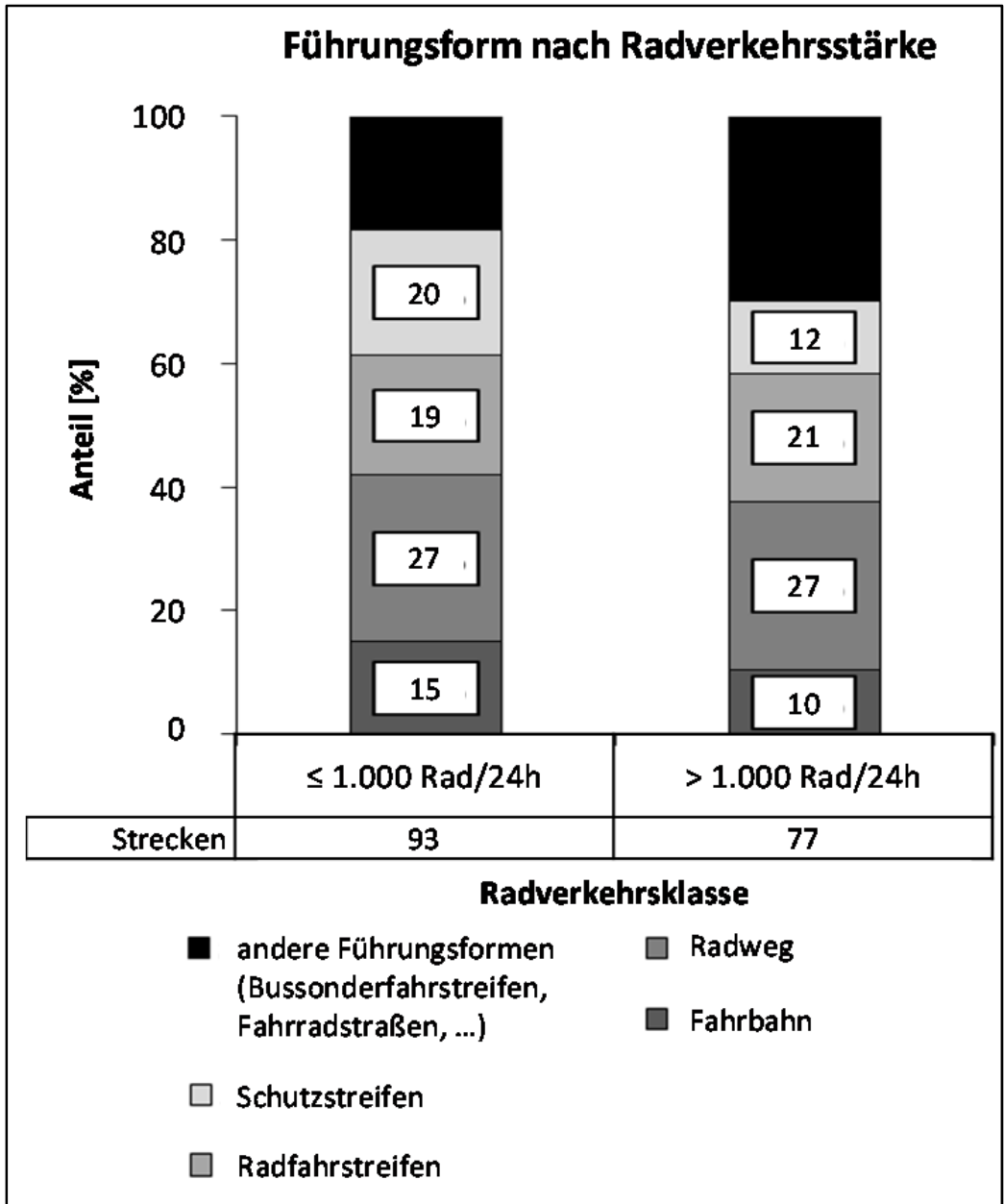

Bild 69: Führungsformen und Radverkehrsstärke in zwei Klassen 


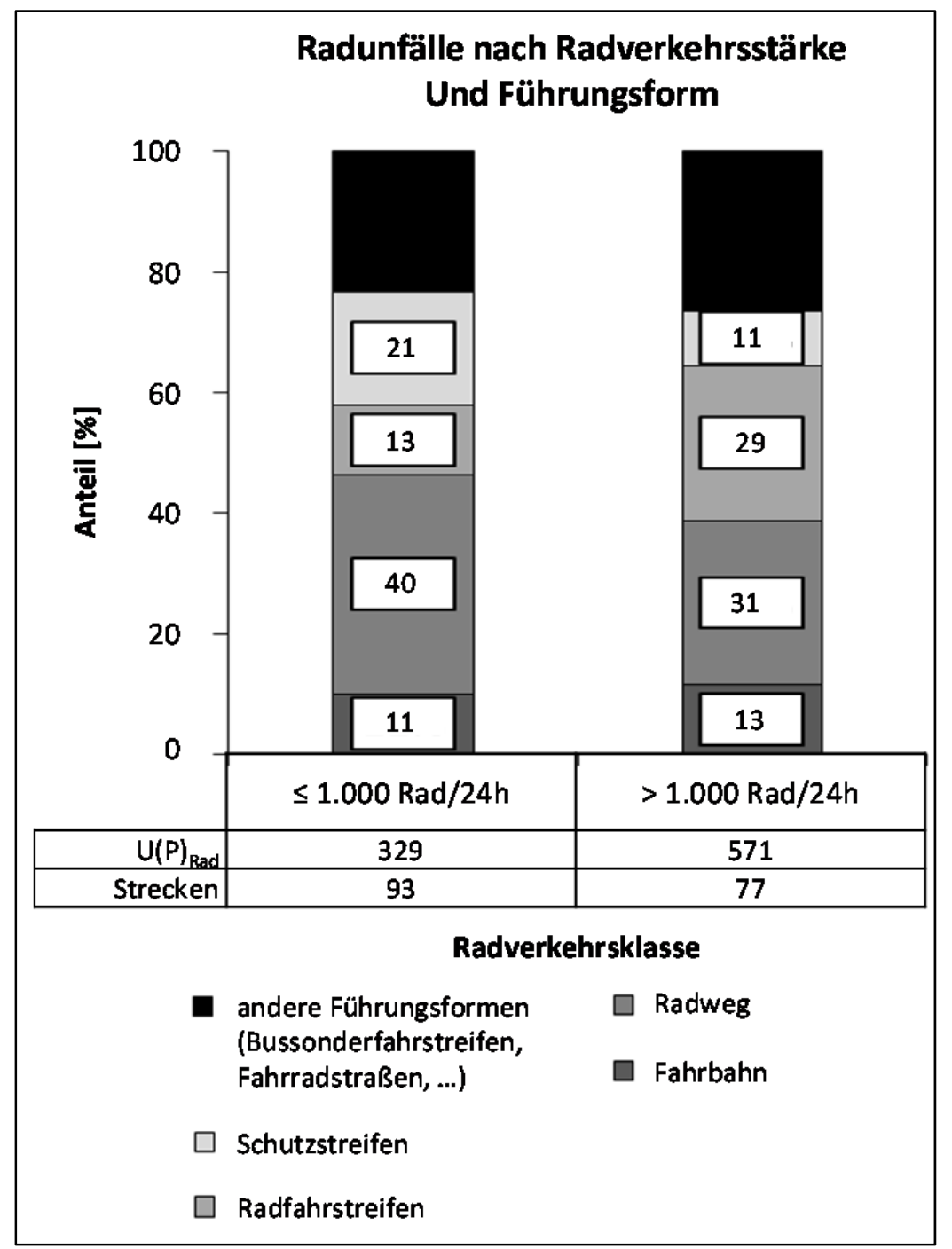

Bild 70: Unfälle nach Führungsformen und Radverkehrsstärke in zwei Klassen 
7.4 Unfalltypenverteilung an Strecken inklusive Unfalltyp „Sonstiger Unfall"

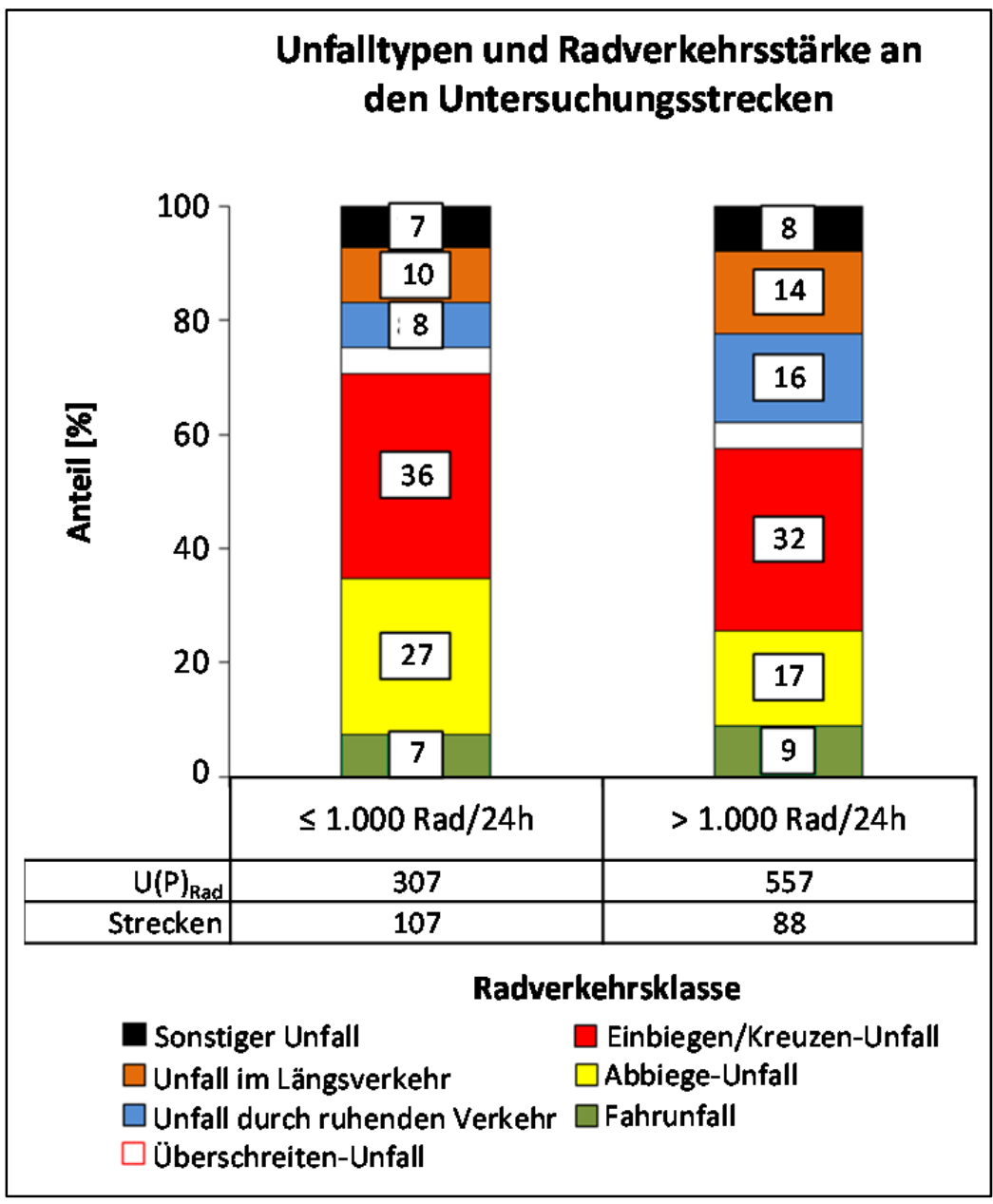

Bild 71: Unfalltypen (Anteile, mit Typ 7) und Radverkehrsstärke in zwei Klassen 


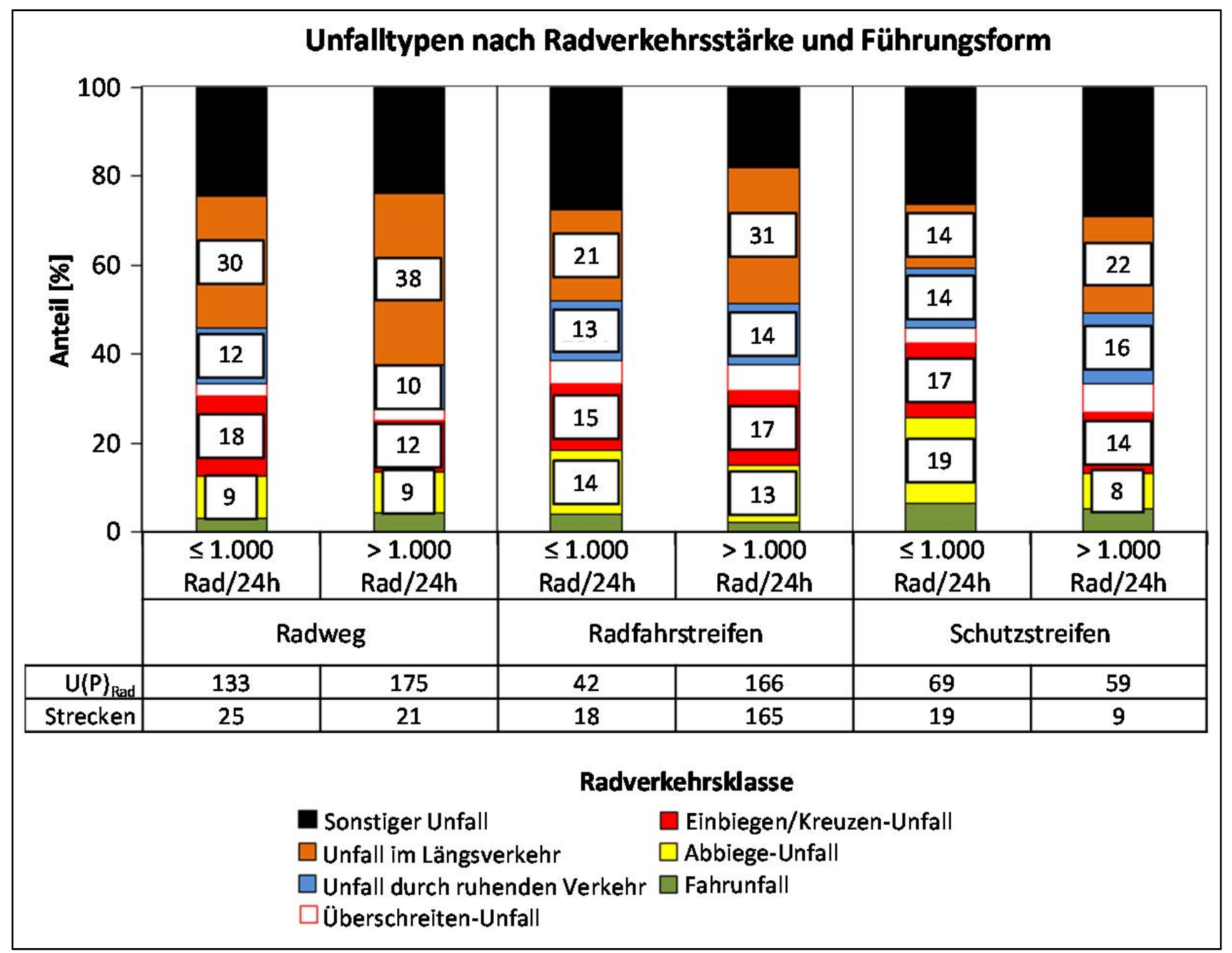

Bild 72: Unfalltypen nach Führungsformen und Radverkehrsstärke in zwei Klassen 


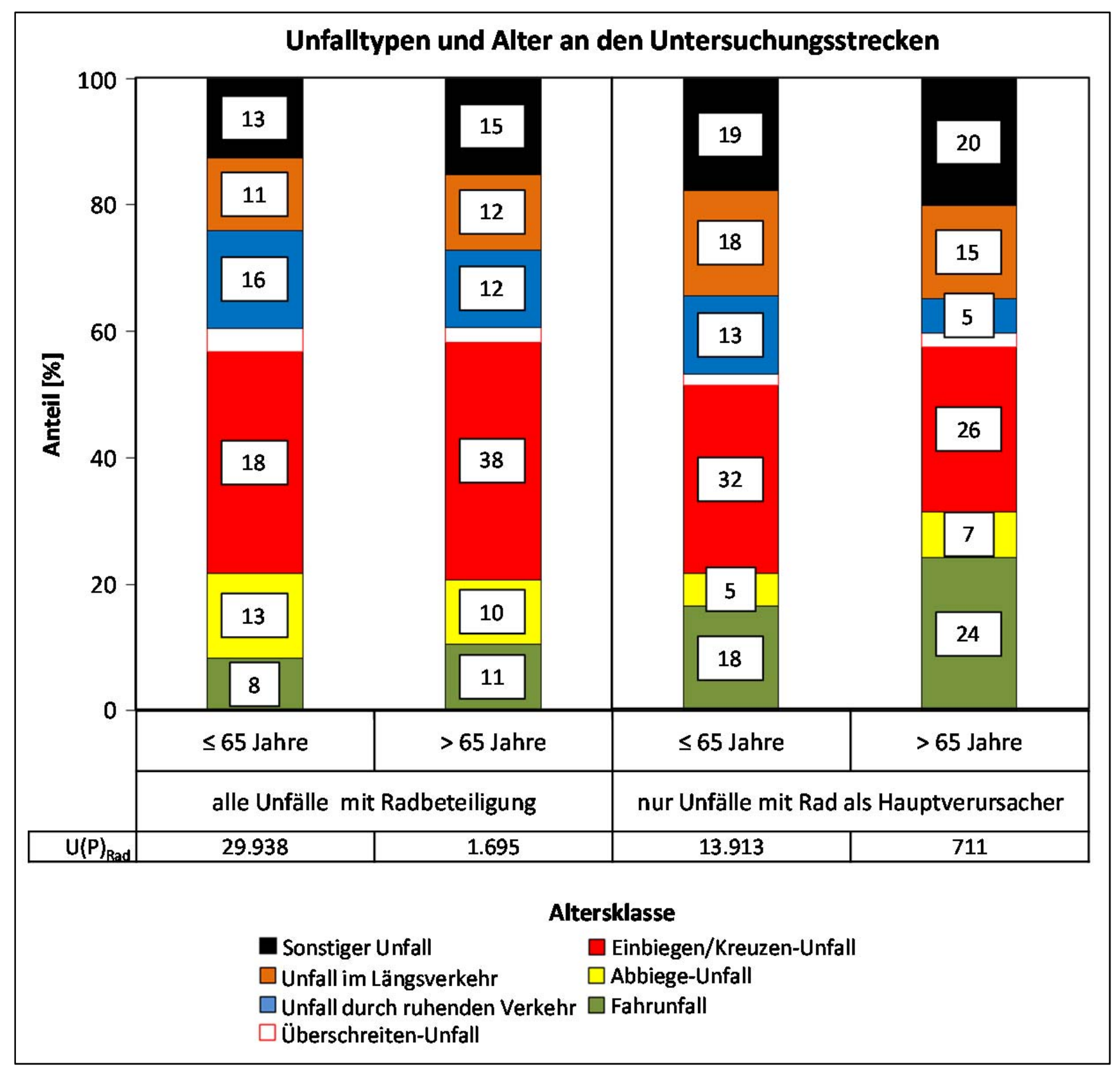

Bild 73:

Unfalltypen (Anteile, mit Typ 7), Verursachung und Alter in zwei Klassen 


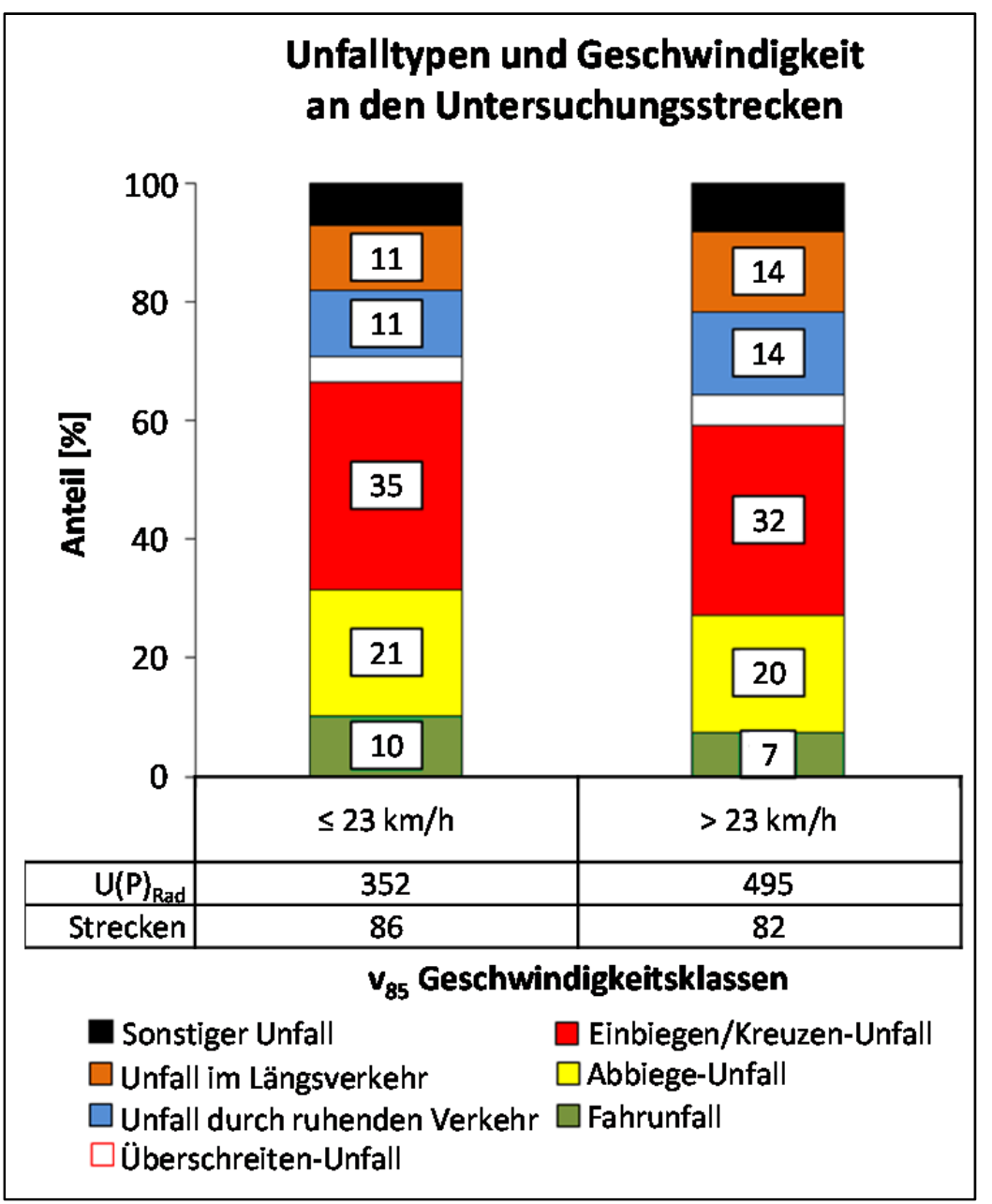

Bild 74: Unfalltypen an Strecken (Anteile, mit Typ 7) und Geschwindigkeit in zwei Klassen 
7.5 Unfalltypenverteilung an Knotenpunkten inklusive Unfalltyp „Sonstiger Unfall“"

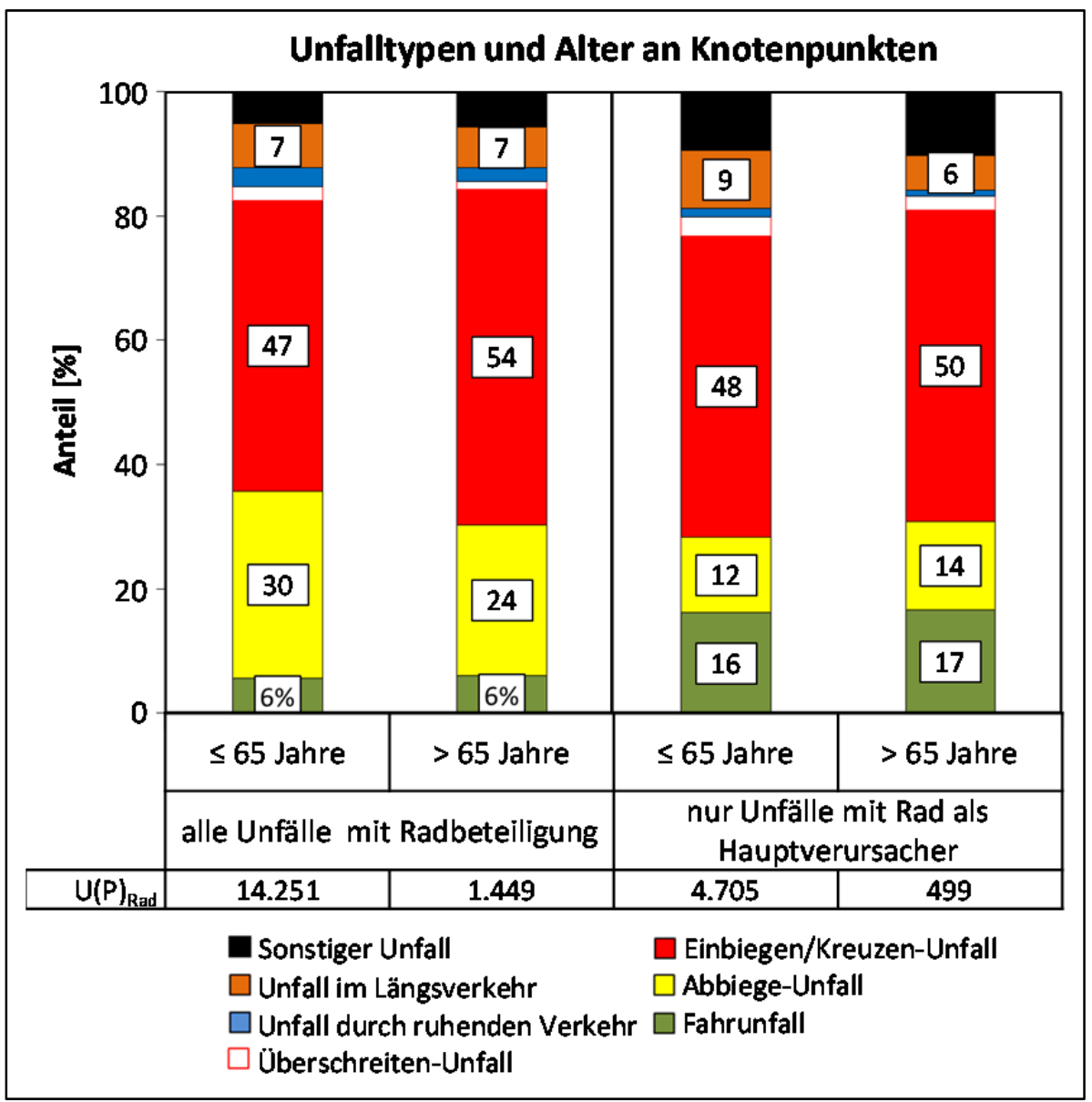

Bild 75: Unfalltypen an Knotenpunkten (Anteile, mit Typ 7), Verursachung und Alter in zwei Klassen 


\subsection{Bedingte und separate Freigabe an Knotenpunkten}

Ein Vergleich von Knotenpunkten, bei denen bedingt verträgliche Fahrrad- und Kfz-Ströme nicht gleichzeitig geführt werden, mit anderen LSA-Knotenpunkten (Untersuchungsstädte Bonn, Troisdorf, Köln, Münster) zeigte, dass

- sich an den untersuchten Stellen die Anteile der Abbiegeunfälle reduzieren und ÜberschreitenUnfälle leicht zunehmen (Bild 76)

- der Hauptverursacheranteil sich zu den Radfahrern verschiebt (weil die häufig PKW-verursachten Abbiegeunfälle wegfallen, Bild 77),

- sich die Unfallschwere reduziert (Anteilsverschiebung in Richtung Unfälle mit Leichtverletzten, Bild 78),

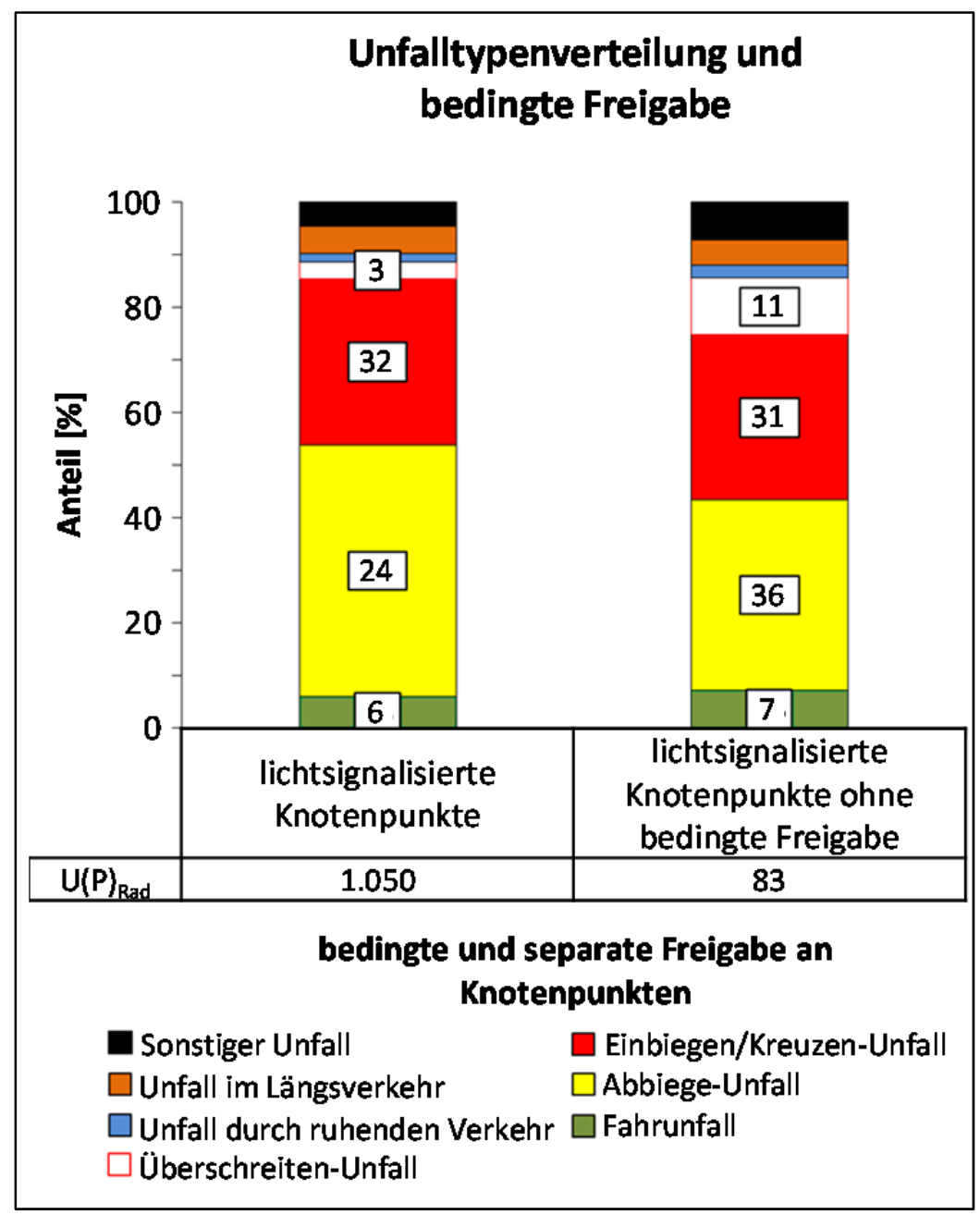

Bild 76: Unfalltypen und bedingte Freigabe 


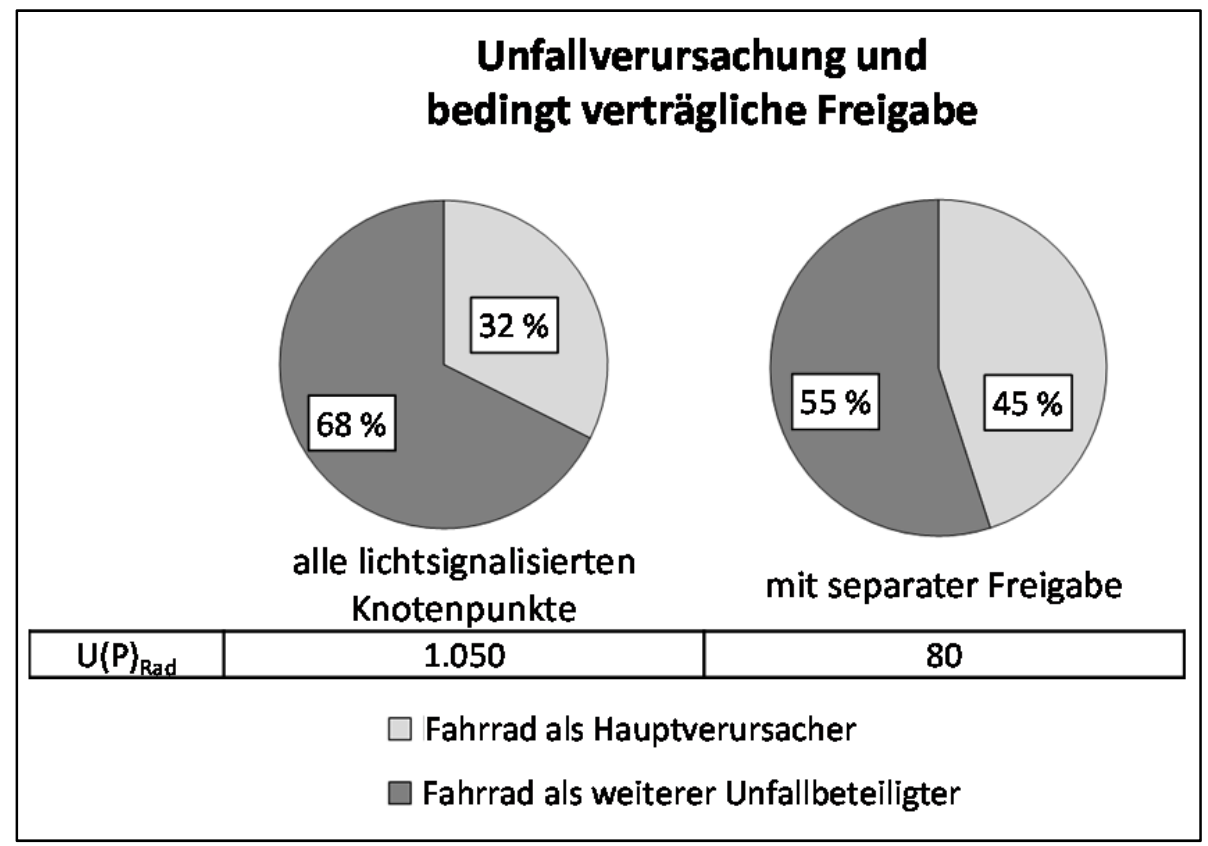

Bild 77: Unfallverursachung und bedingte Freigabe

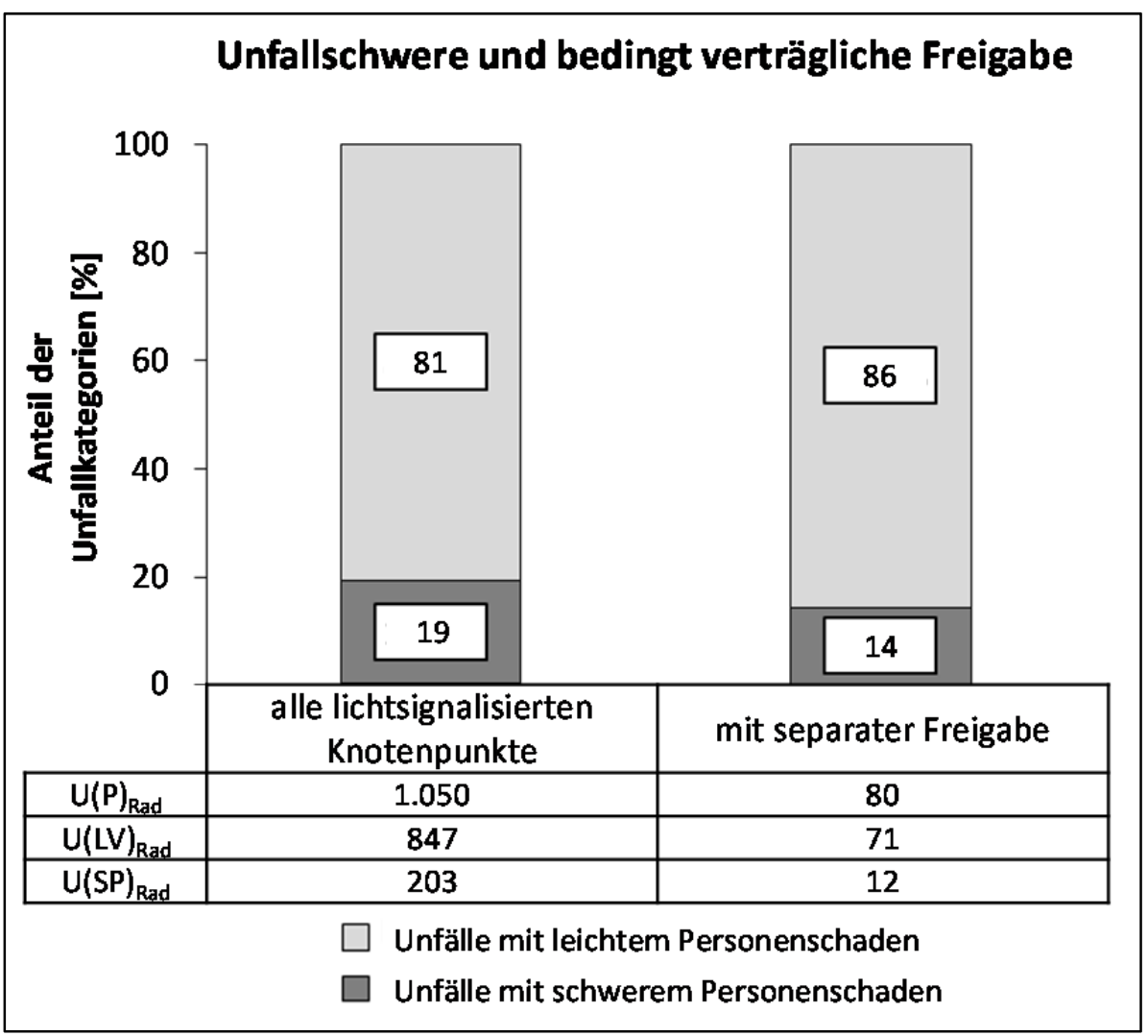

Bild 78: Unfallschwere und bedingte Freigabe

Demnach hilft die separate Freigabe des Abbiegeverkehrs und des in Hauptrichtung fahrenden Radfahrers die Abbiegeunfälle anteilig zu reduzieren.

Die Unfälle, die an Knotenpunkten mit separater Freigabe beider Ströme stattfanden, wurden häufiger von Radfahrern verursacht - häufig im Zusammenhang mit „Nichtbeachten der Lichtzeichen“ (Ursache 31) oder Verkehrsuntüchtigkeit durch Alkoholeinfluss des Radfahrers (Ursache 1).

Des Weiteren reduzierte sich an diesen Knotenpunkten der Anteil der Radverkehrsunfälle mit schwerem Personenschaden. 


\subsection{Berechnung der durchschnittlichen Abweichungen der Radfahrer- geschwindigkeiten}

Die Berechnung der durchschnittlichen Abweichungen der Radfahrergeschwindigkeiten sei hier an einem Beispiel erläutert: Im Mischverkehr fuhren Erwachsene der mittleren Altersgruppe mit unterschiedlichen Fahrradtypen durchschnittlich zwischen 18 und $25 \mathrm{~km} / \mathrm{h}$. Die mittlere Geschwindigkeit dieser Altersgruppe im Mischverkehr über alle Fahrradtypen betrugt $20,4 \mathrm{~km} / \mathrm{h} .{ }^{56}$ Hiervon wich die mittlere Geschwindigkeit z. B. mit einem Rennrad um 4,2 km/h, mit einem Holland-Rad um 2,3 km ab. Die durchschnittliche Abweichung der Geschwindigkeiten dieser Radfahrer mit unterschiedlichen Fahrradtypen (,average-derivation“, AD-Streuung) betrug 2,4 km/h (Tabelle 56).

Tabelle 56: Beispiel für die Ermittlung einer AD-Streuung

\begin{tabular}{|c|c|c|c|c|c|c|c|c|c|c|}
\hline \multirow{2}{*}{ 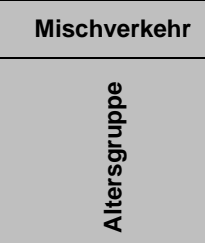 } & \multicolumn{4}{|c|}{$\begin{array}{l}\text { durchschnittliche Geschwindigkeit } \\
\text { nach Fahrradtyp [km/h] }\end{array}$} & \multirow[b]{2}{*}{$\begin{array}{c}\text { mittlere Geschwin- } \\
\text { digkeit aller Fahr- } \\
\text { radtypen }[\mathrm{km} / \mathrm{h}]\end{array}$} & \multicolumn{4}{|c|}{$\begin{array}{l}\text { Differenz zu mittlerer Geschwindigkeit aller Fahrrad- } \\
\text { typen }[\mathrm{km} / \mathrm{h}]\end{array}$} & \multirow[b]{2}{*}{$\begin{array}{l}\text { AD- } \\
\text { Streuung }\end{array}$} \\
\hline & 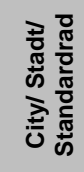 & 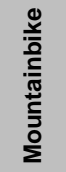 & 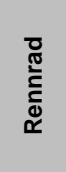 & 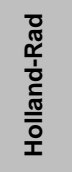 & & 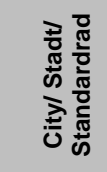 & 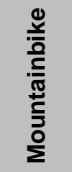 & 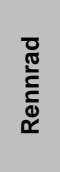 & 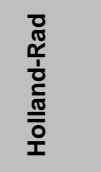 & \\
\hline 25-65 Jahre & 18,9 & 20,0 & 24,6 & 18,1 & 20,4 & $-1,5$ & $-0,4$ & 4,2 & $-2,3$ & 2,1 \\
\hline N Rf & 1157 & 94 & 38 & 42 & & & & & & \\
\hline
\end{tabular}

Wurden nun z. B. die verschiedenen Altersgruppen und Anlagentypen konstant gehalten und der Fahrradtyp als unabhängige Variable herangezogen, wichen die Geschwindigkeiten durchschnittlich um $1,0 \mathrm{~km} / \mathrm{h}$ ab. 


\subsection{Fahrlinien von Radfahrern an lichtsignalgeregelten Knotenpunkten}

\subsubsection{Linksabbieger}

Tabelle 57: Fahrlinien linksabbiegender Radfahrer mit Radwegzufahrt

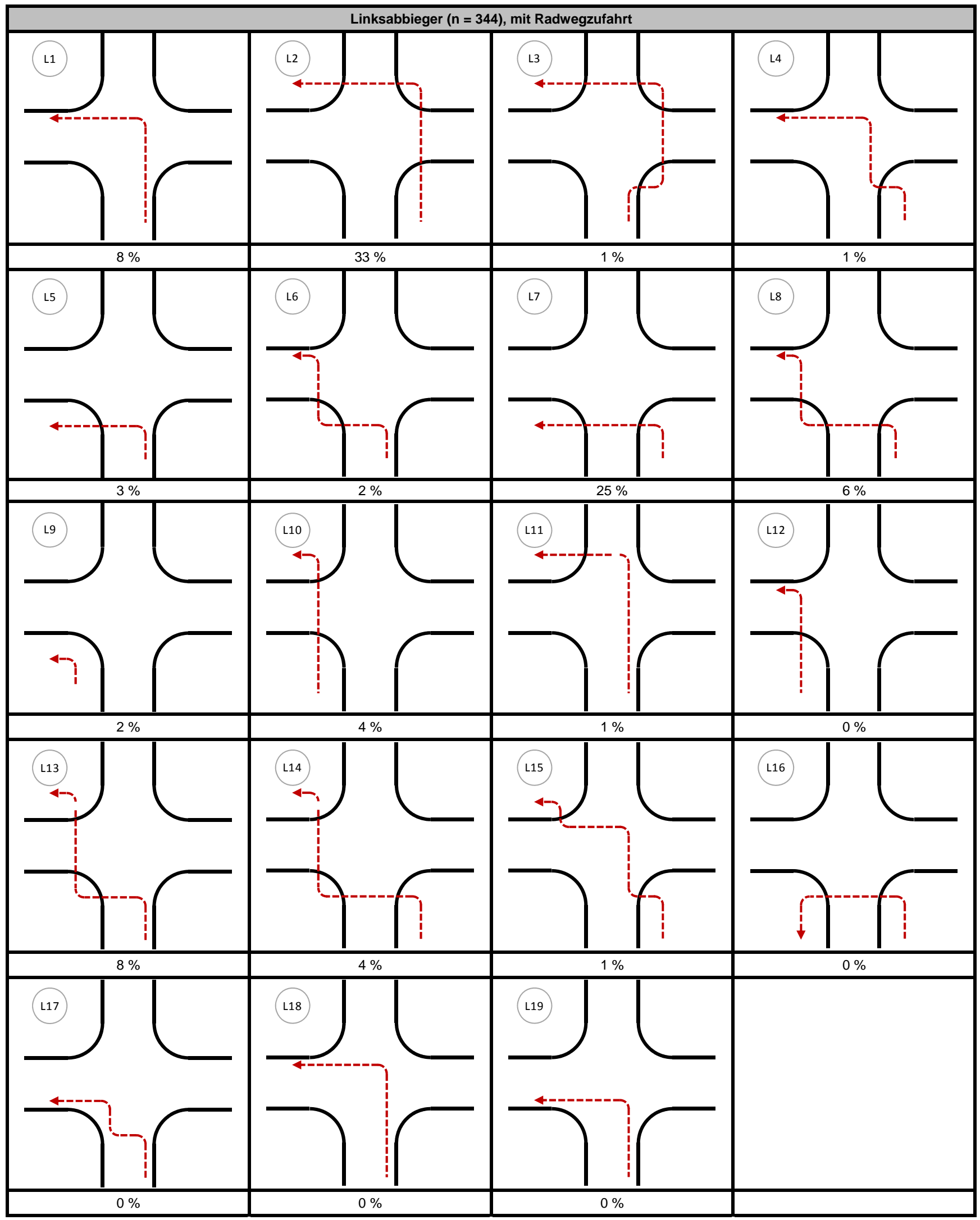

Tabelle 58: Fahrlinien linksabbiegender Radfahrer mit Radwegzufahrt bei nicht abgesetzter Furt 


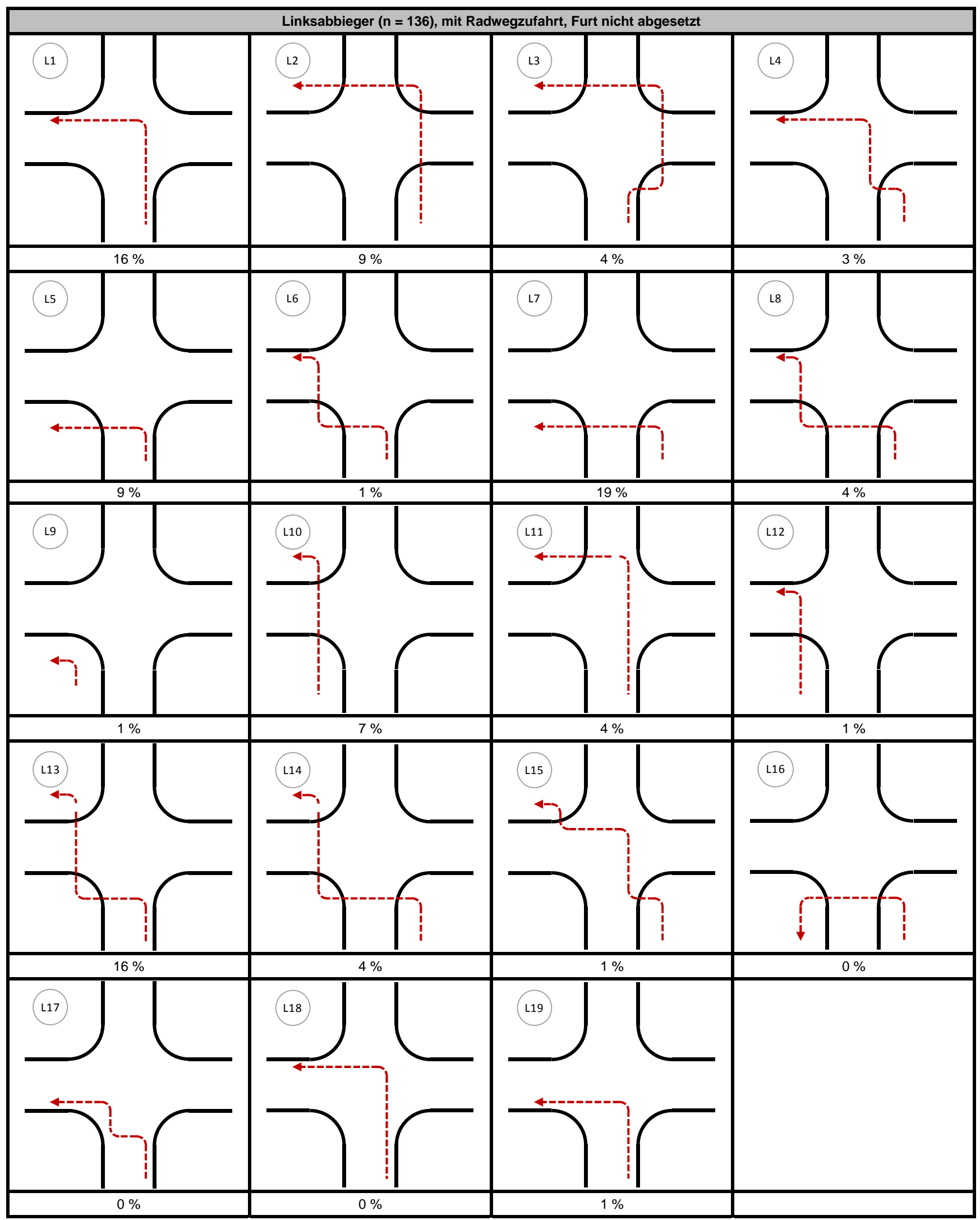


Tabelle 59: Fahrlinien linksabbiegender Radfahrer mit Radwegzufahrt bei abgesetzter Furt

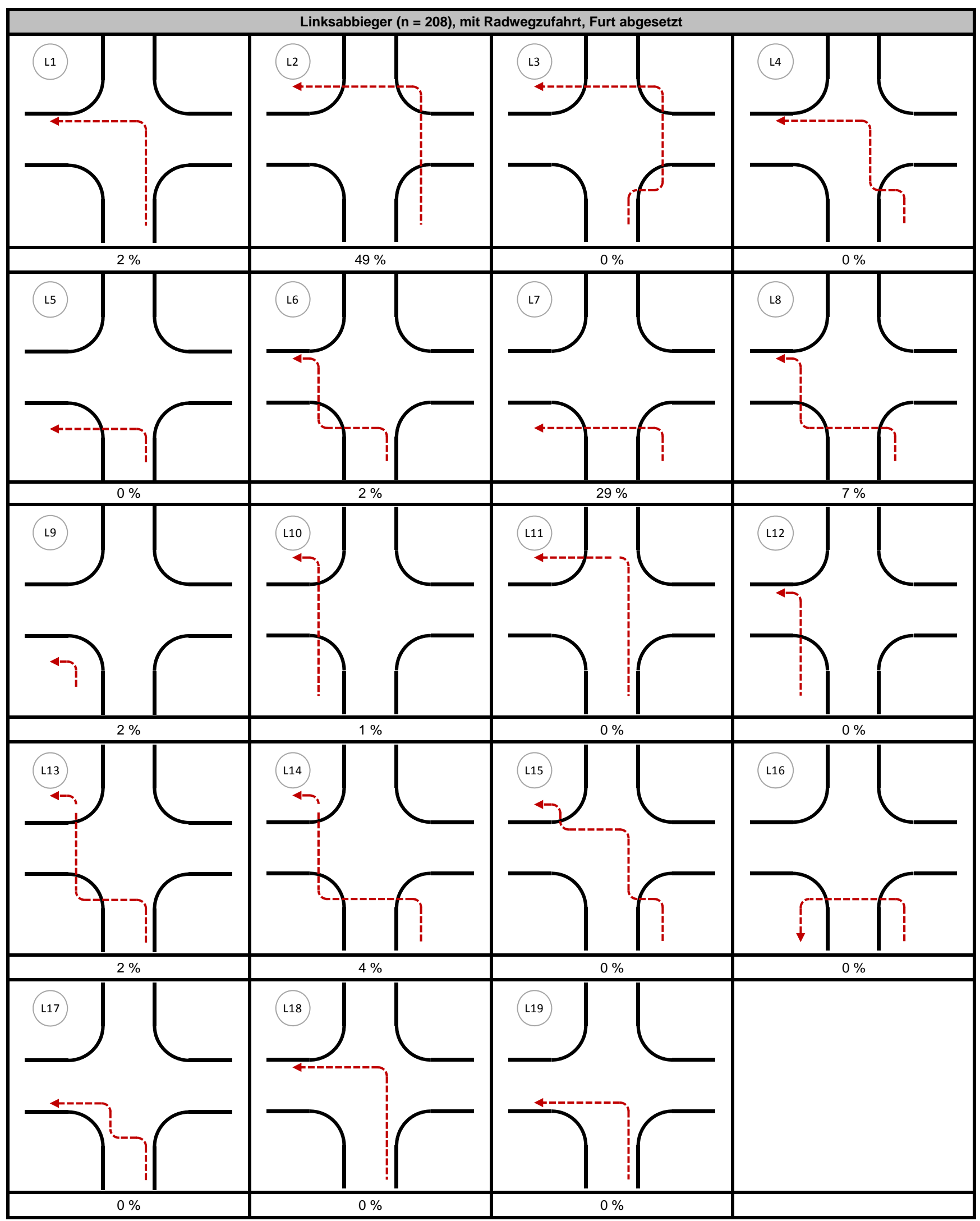


Tabelle 60: Fahrlinien linksabbiegender Radfahrer mit zuführendem Radfahrstreifen

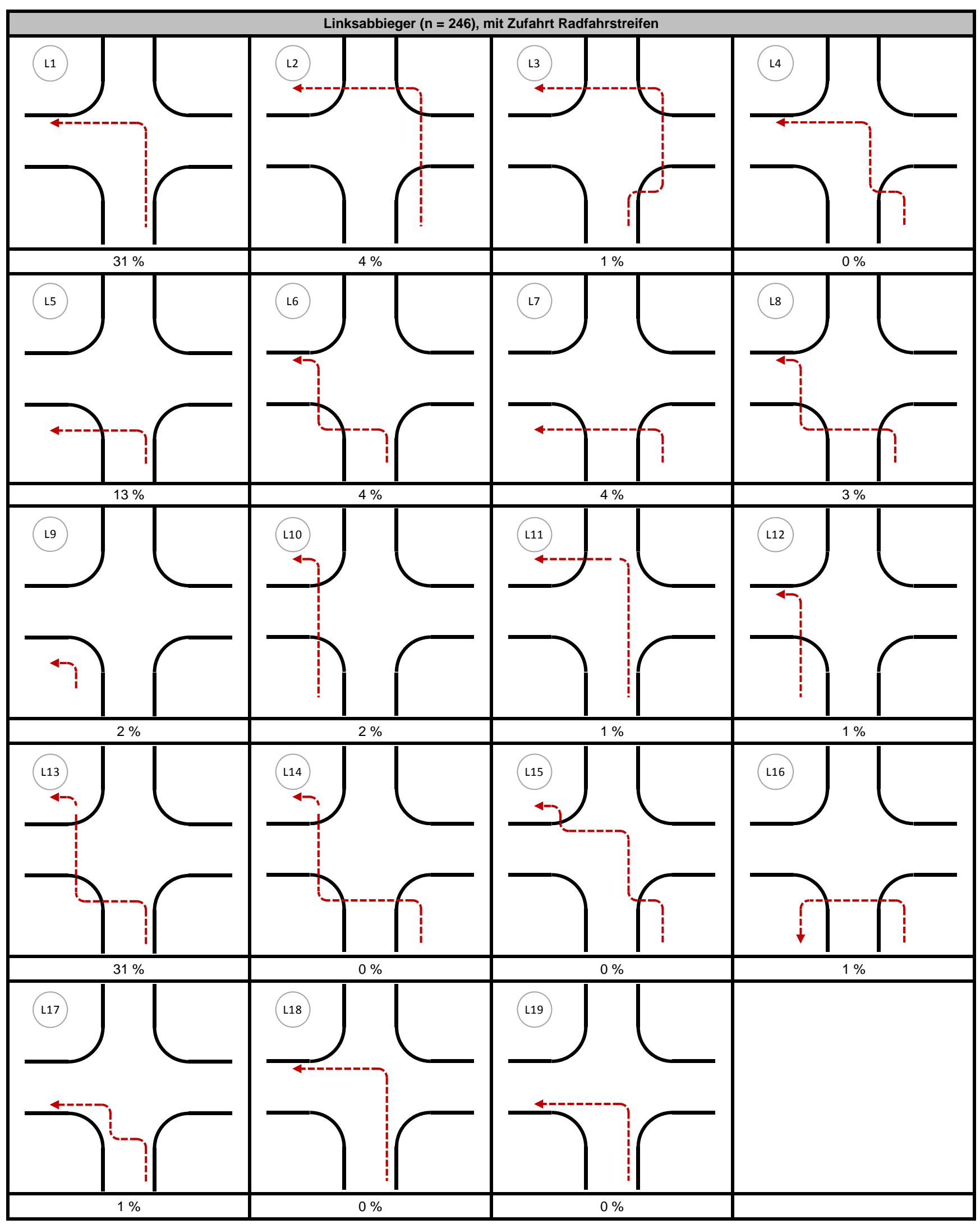


Tabelle 61: Fahrlinien linksabbiegender Radfahrer mit zuführendem Radschutzstreifen

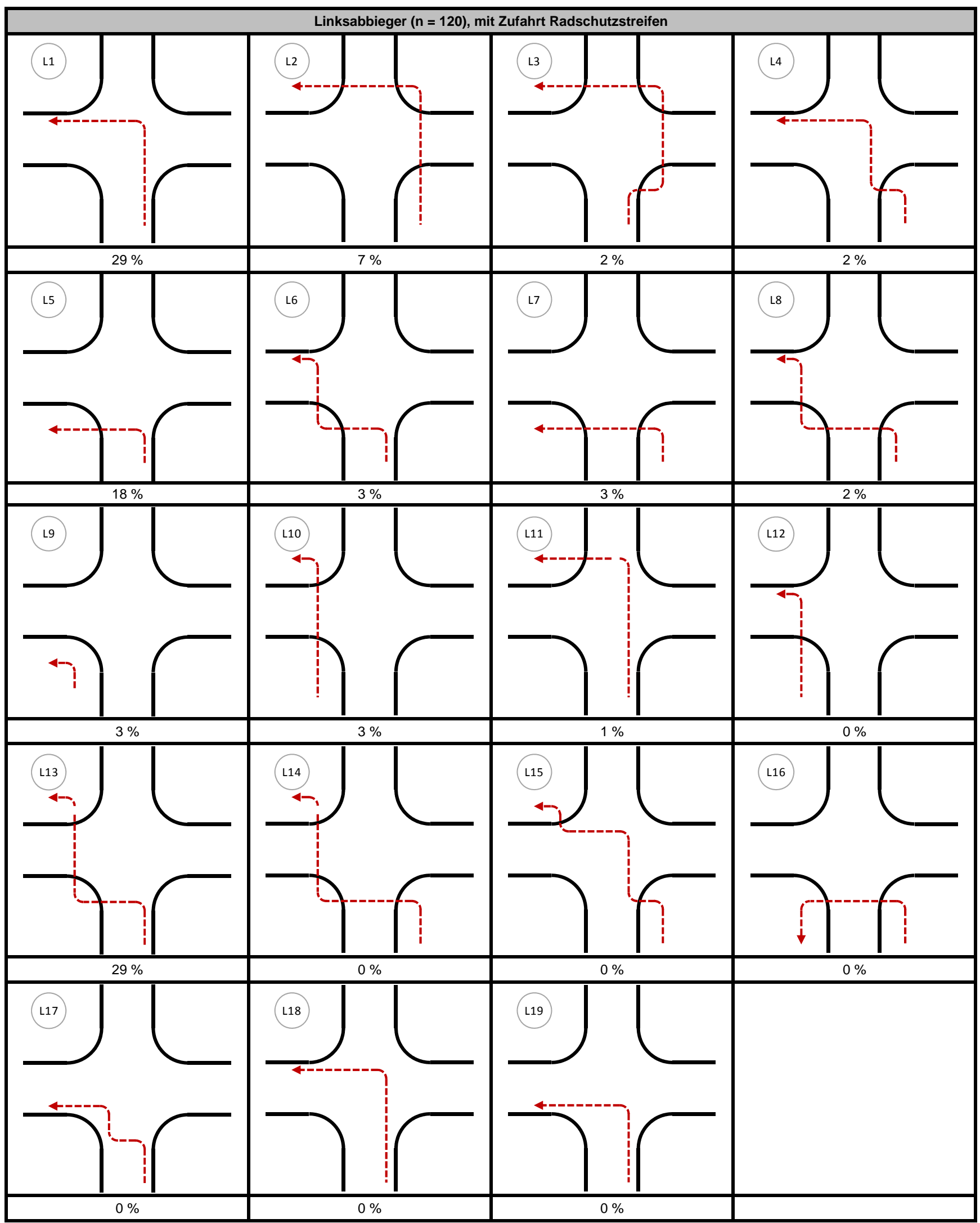


Tabelle 62: Fahrlinien linksabbiegender Radfahrer mit Führung des Radverkehrs auf der Fahrbahn

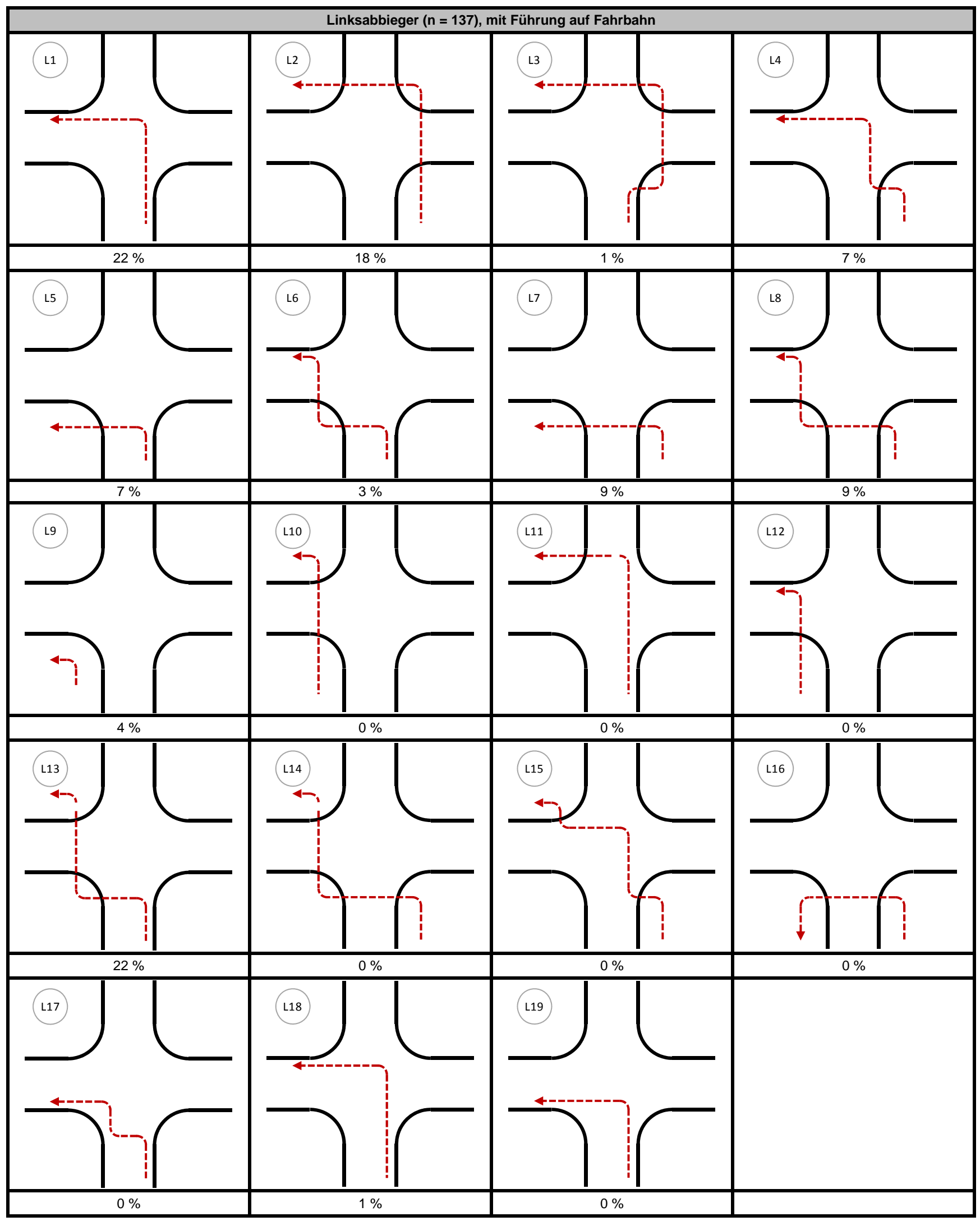




\subsubsection{Rechtsabbieger}

Tabelle 63: Fahrlinien rechtsabbiegender Radfahrer mit Radwegzufahrt

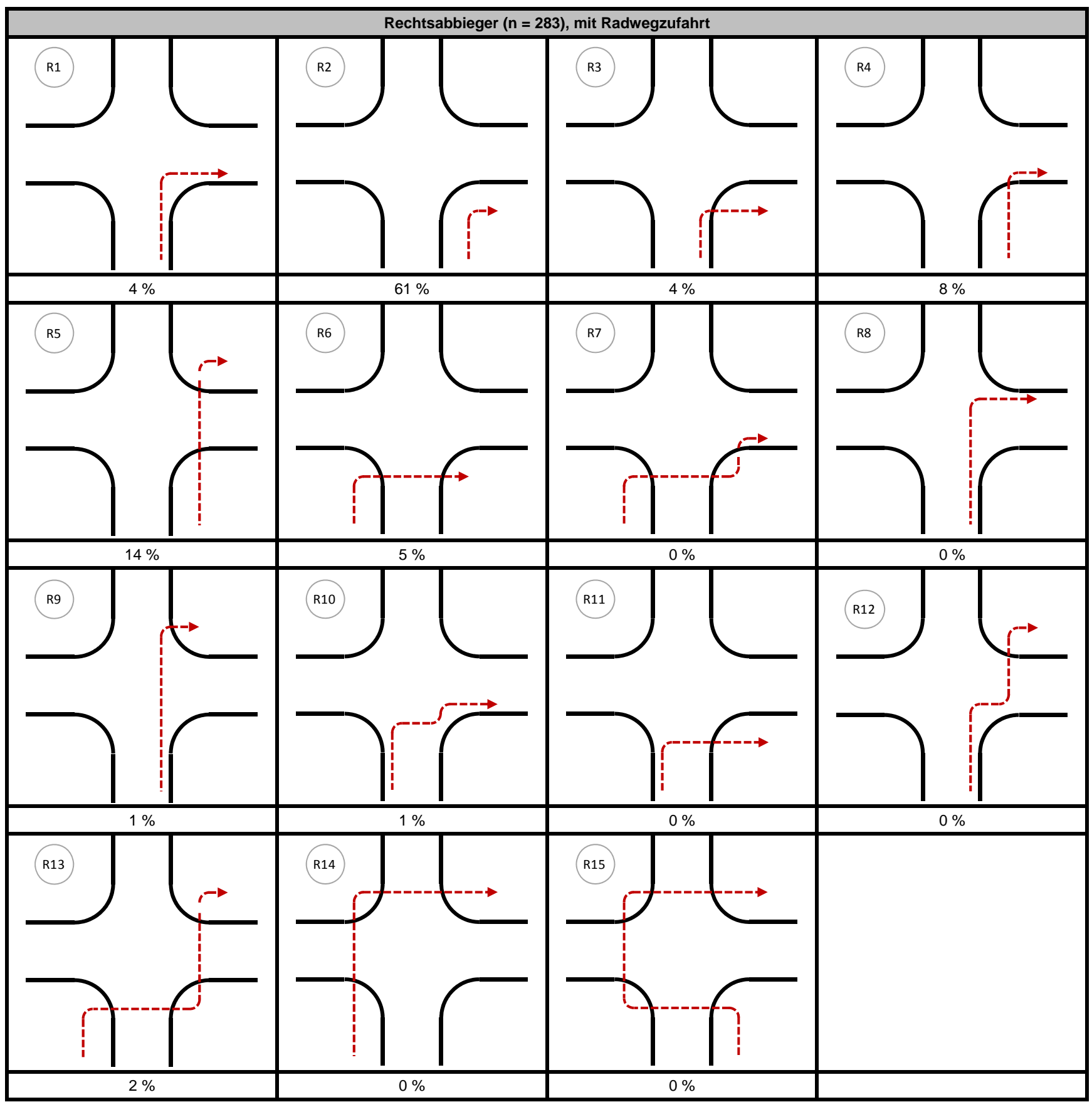


Tabelle 64: Fahrlinien rechtsabbiegender Radfahrer mit Radwegzufahrt bei nicht abgesetzter Furt

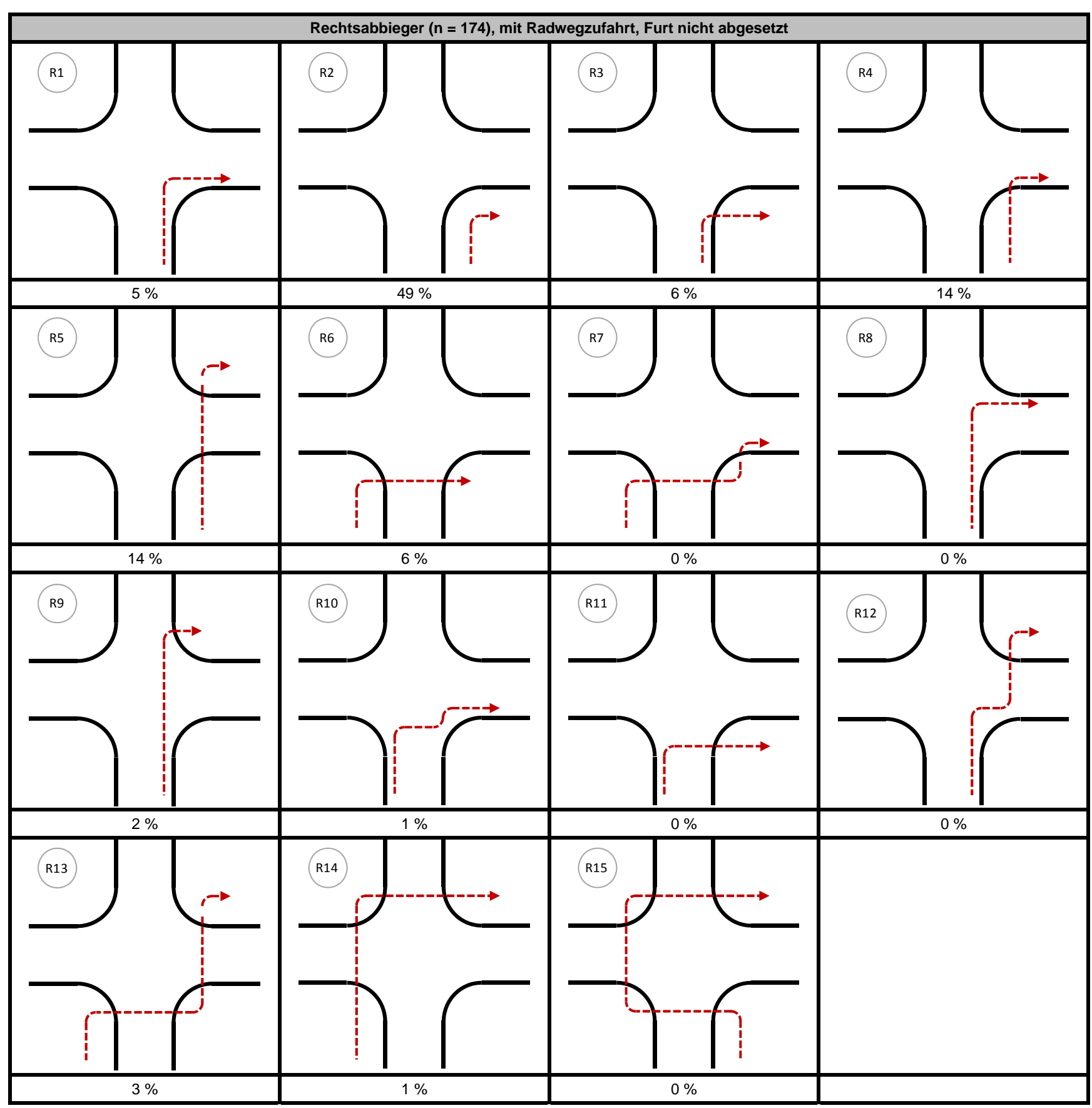


Tabelle 65: Fahrlinien rechtsabbiegender Radfahrer mit Radwegzufahrt bei abgesetzter Furt

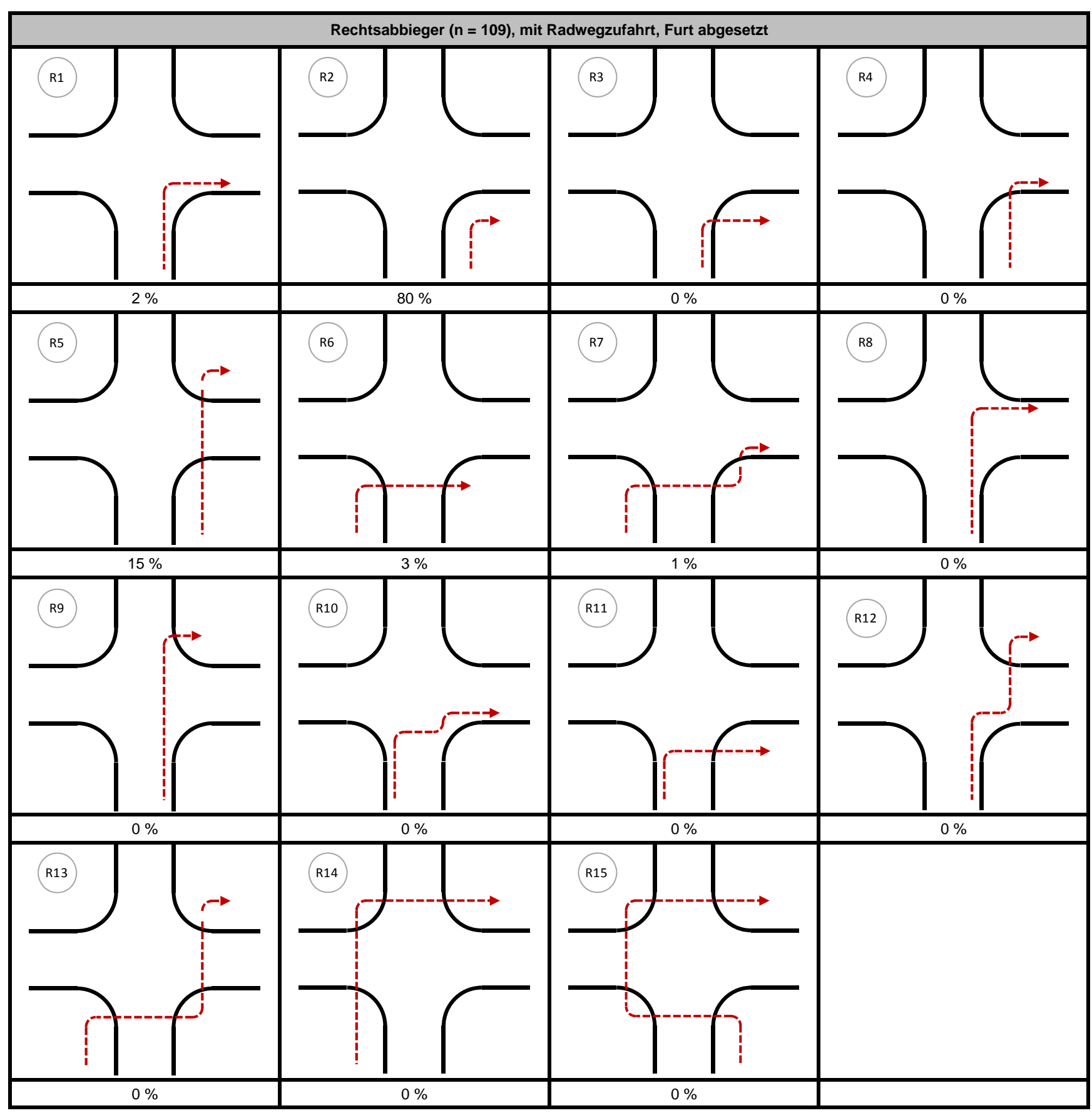


Tabelle 66: Fahrlinien rechtsabbiegender Radfahrer mit zuführendem Radfahrstreifen

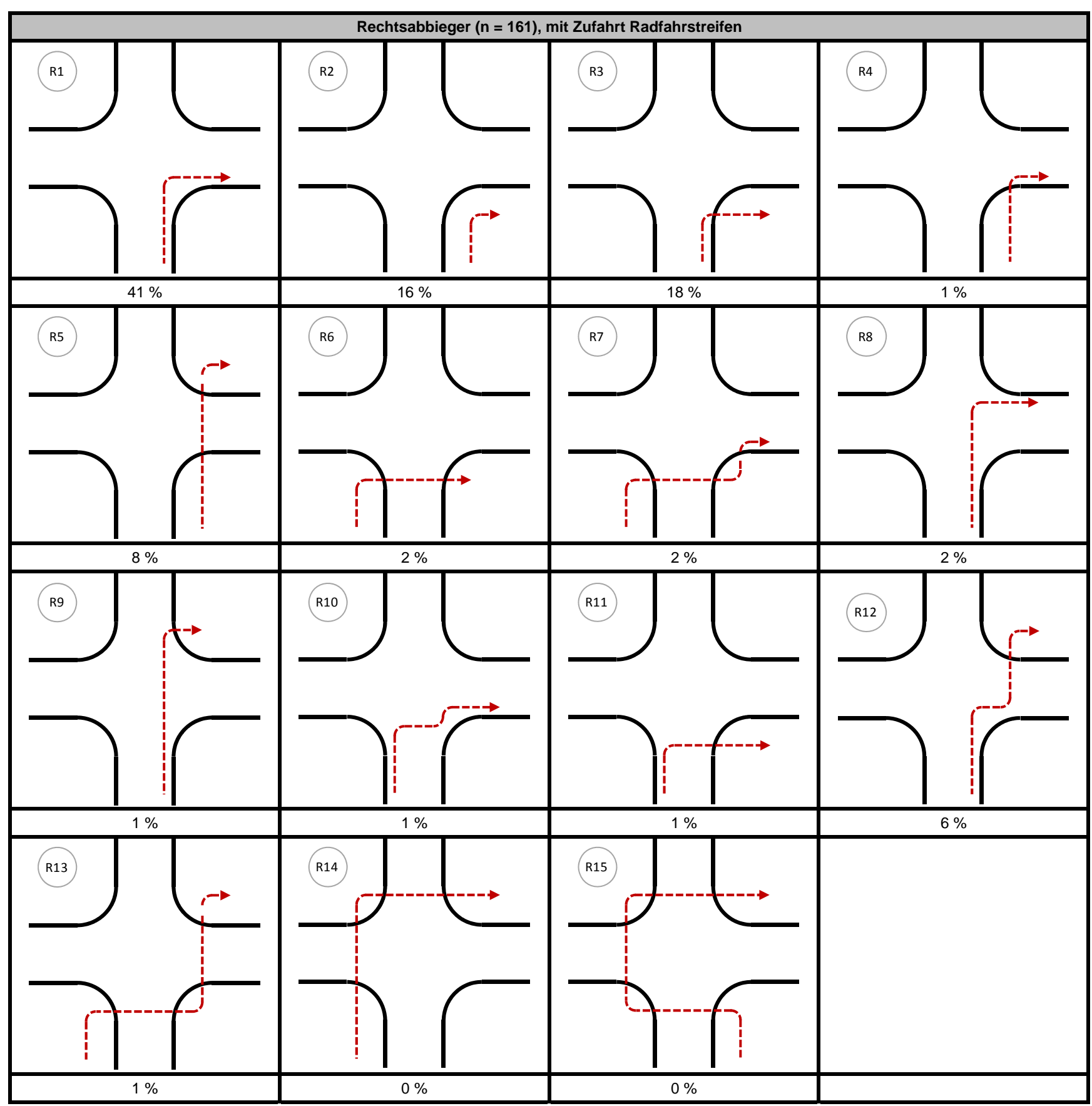


Tabelle 67: Fahrlinien rechtsabbiegender Radfahrer mit zuführendem Radschutzstreifen

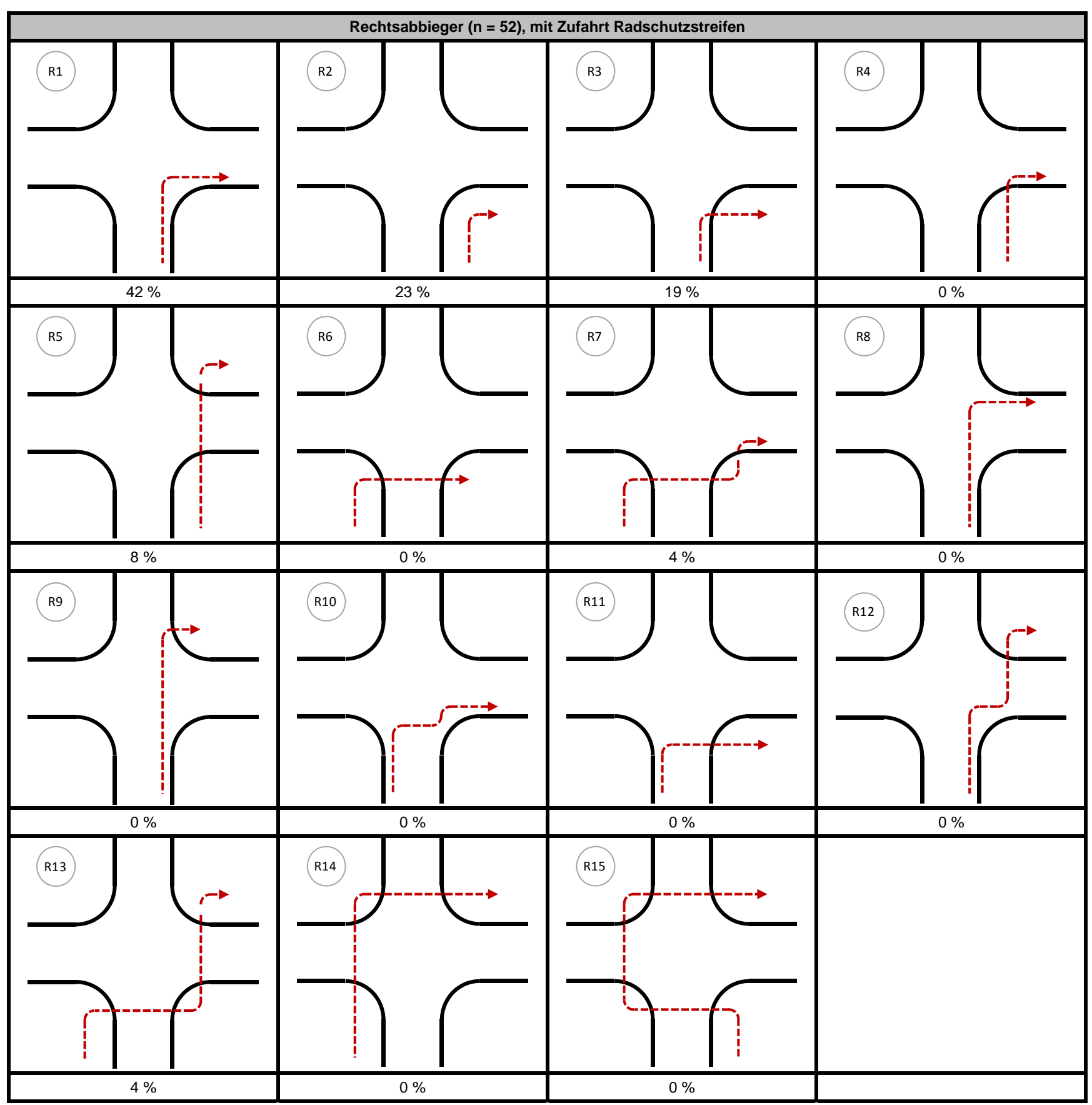


Tabelle 68: Fahrlinien rechtsabbiegender Radfahrer mit Führung des Radverkehrs auf der Fahrbahn

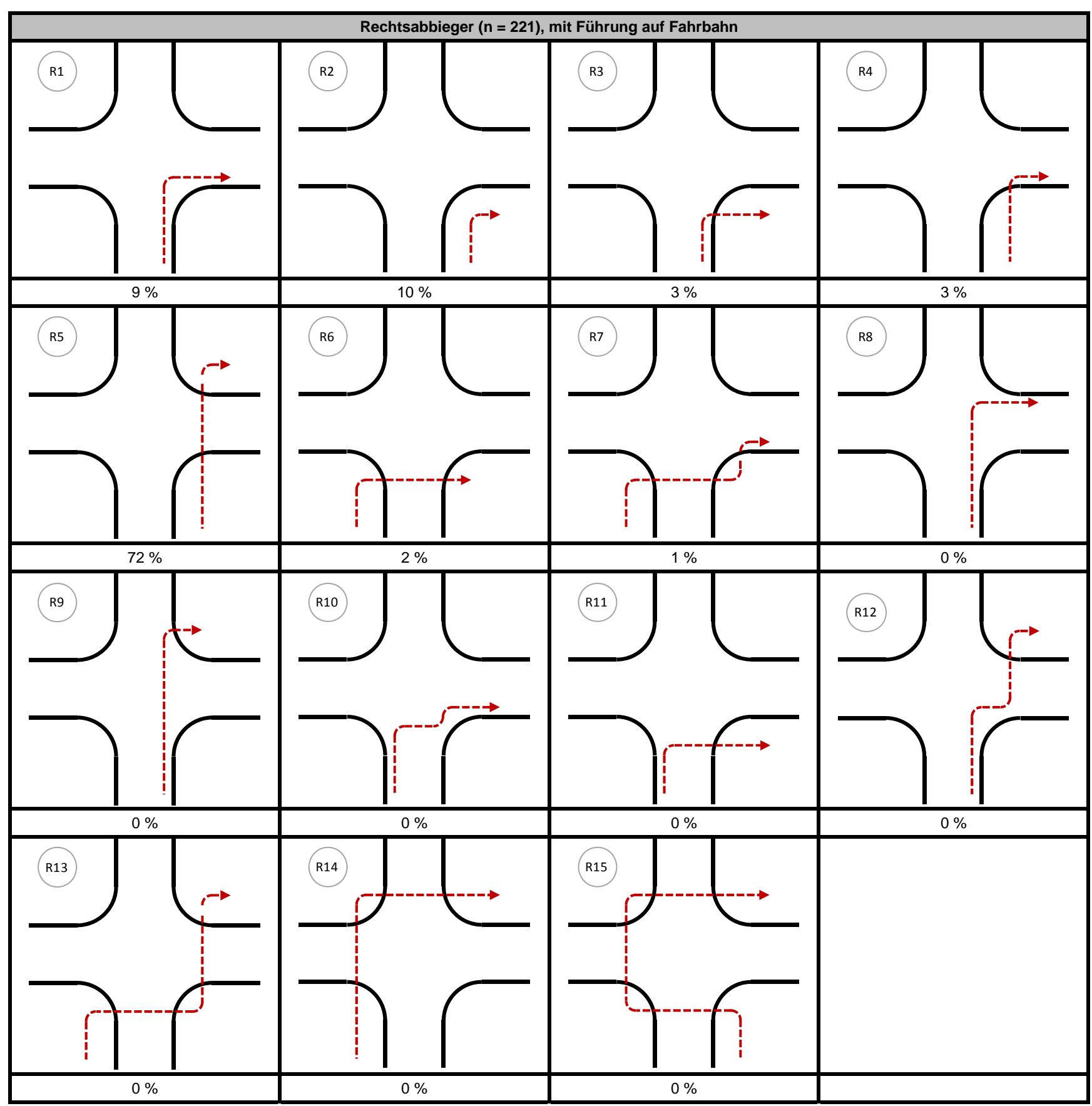


173

7.8.3. Geradeausfahrer

Tabelle 69: Fahrlinien geradeausfahrender Radfahrer mit Radwegzufahrt

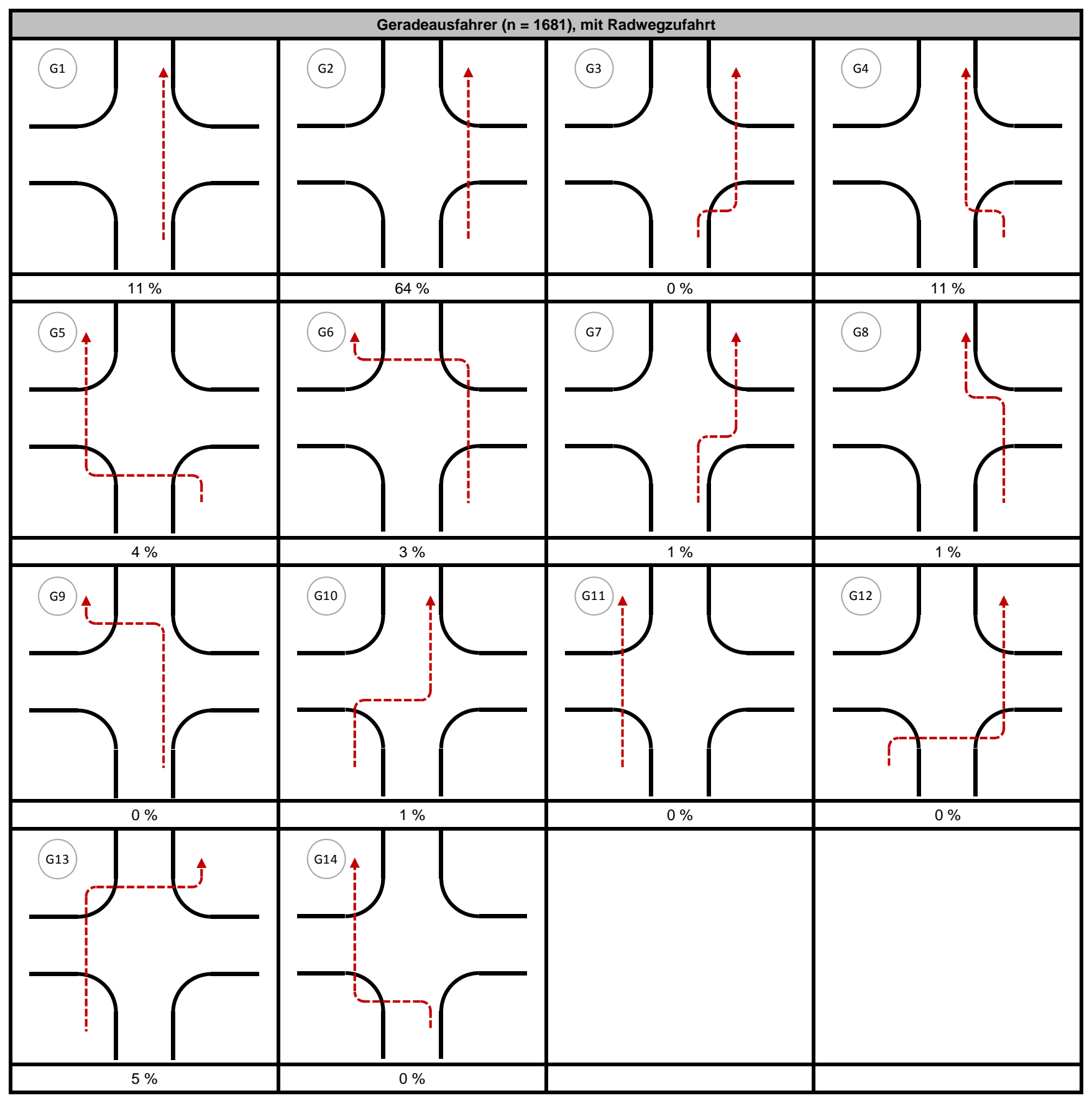


Tabelle 70: Fahrlinien geradeausfahrender Radfahrer mit Radwegzufahrt bei nichtabgesetzter Furt

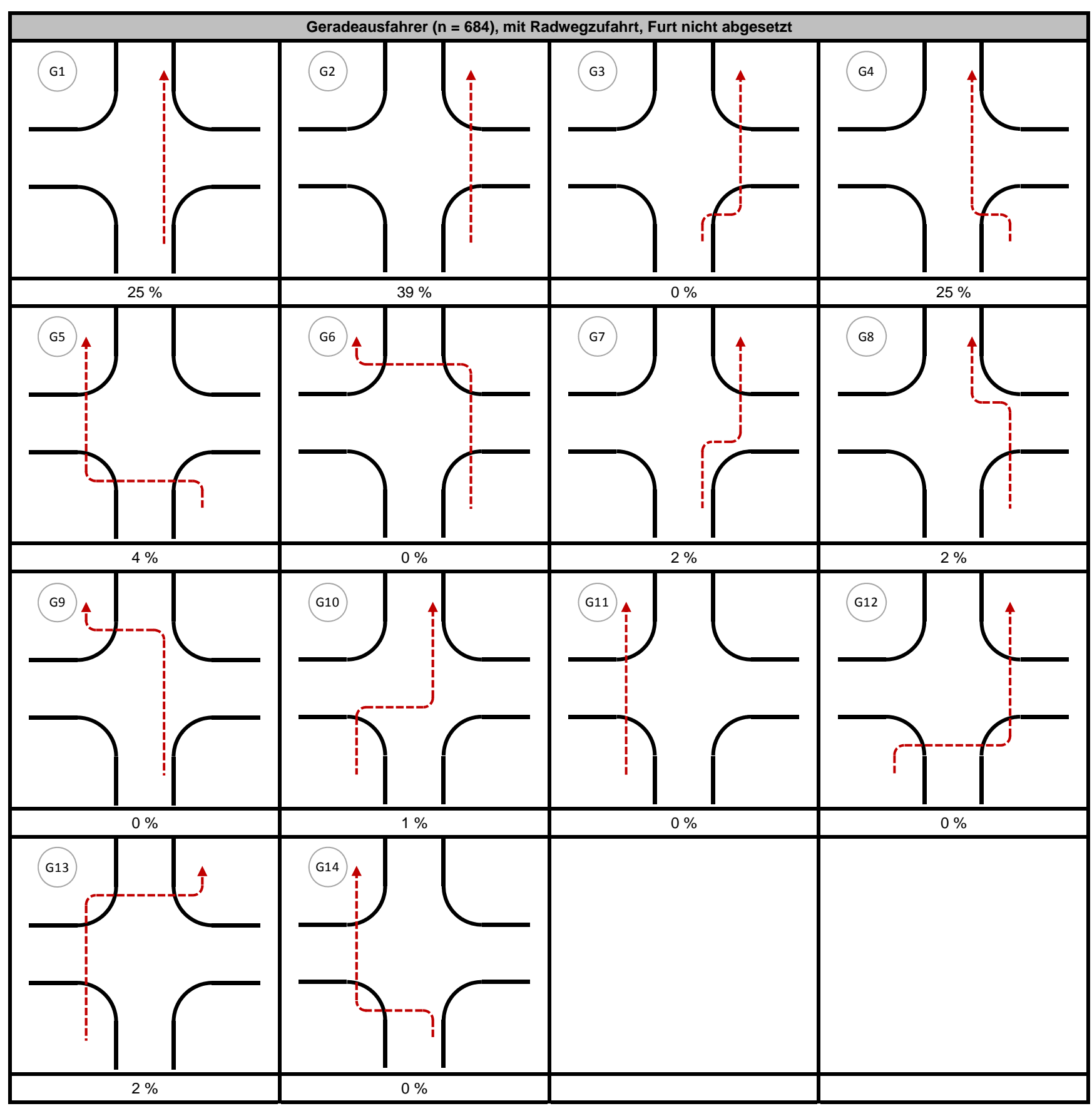


Tabelle 71: Fahrlinien geradeausfahrender Radfahrer mit Radwegzufahrt bei abgesetzter Furt

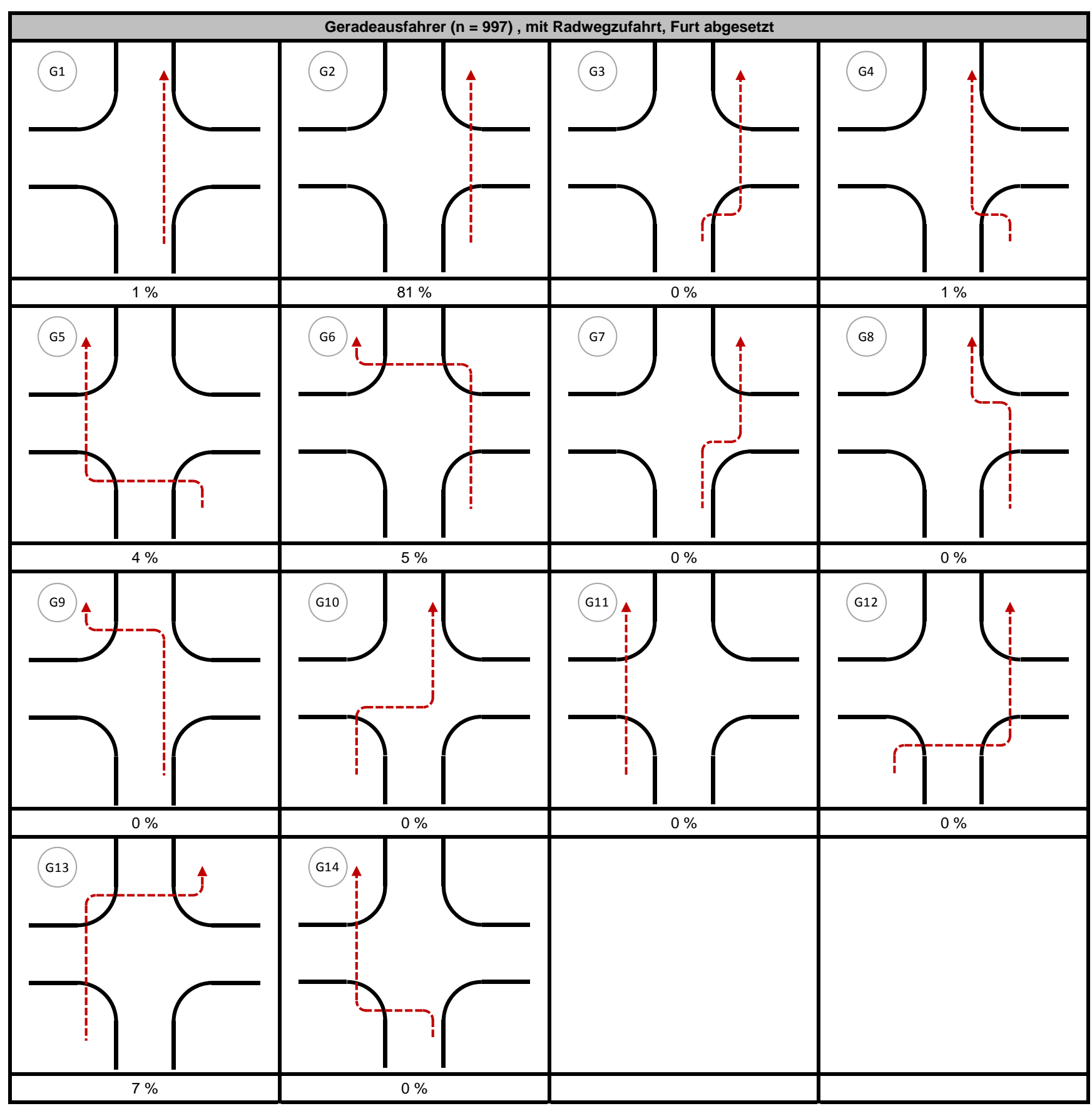


Tabelle 72: Fahrlinien geradeausfahrender Radfahrer mit zuführendem Radfahrstreifen

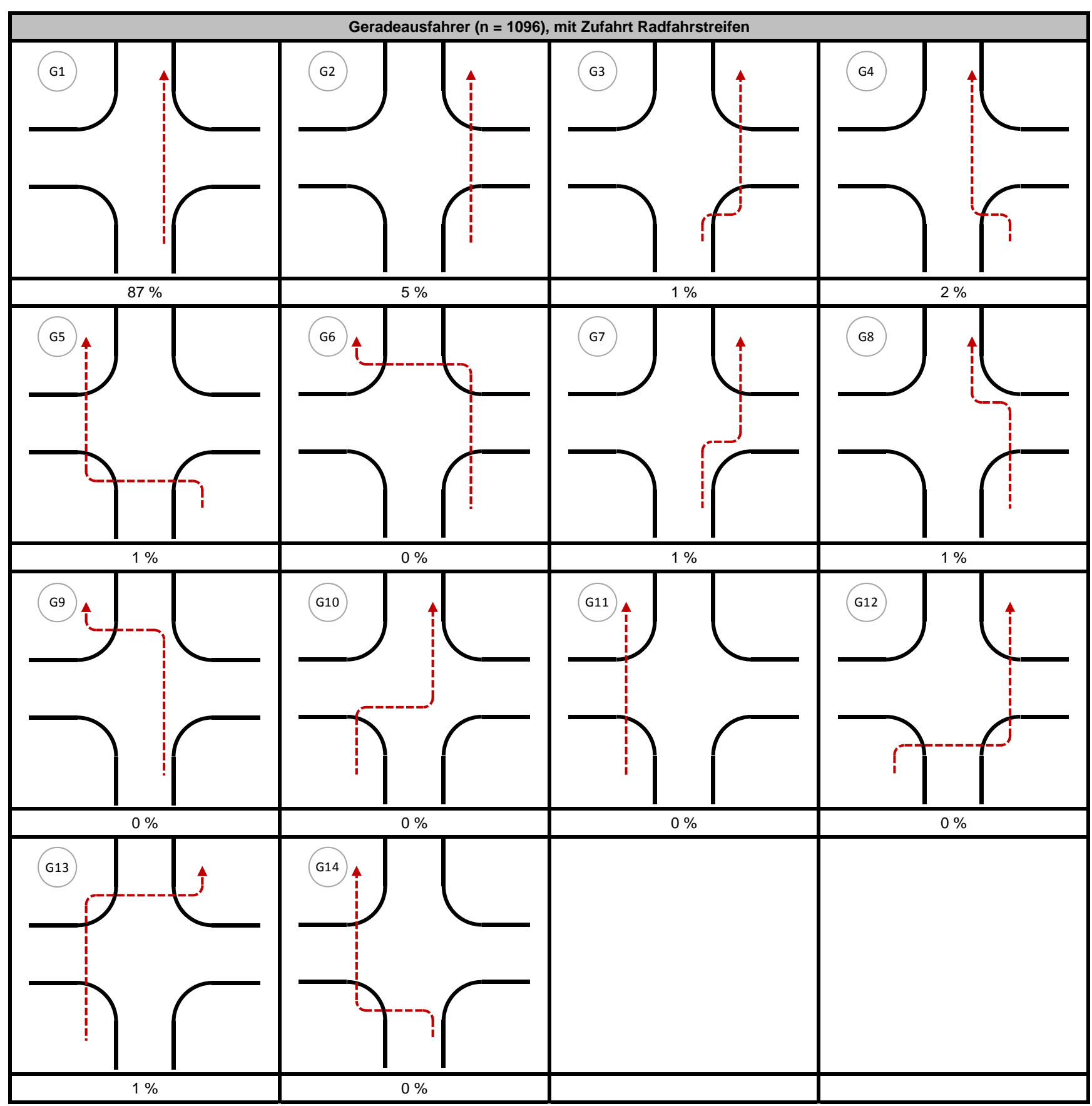


Tabelle 73: Fahrlinien geradeausfahrender Radfahrer mit zuführendem Radschutzstreifen

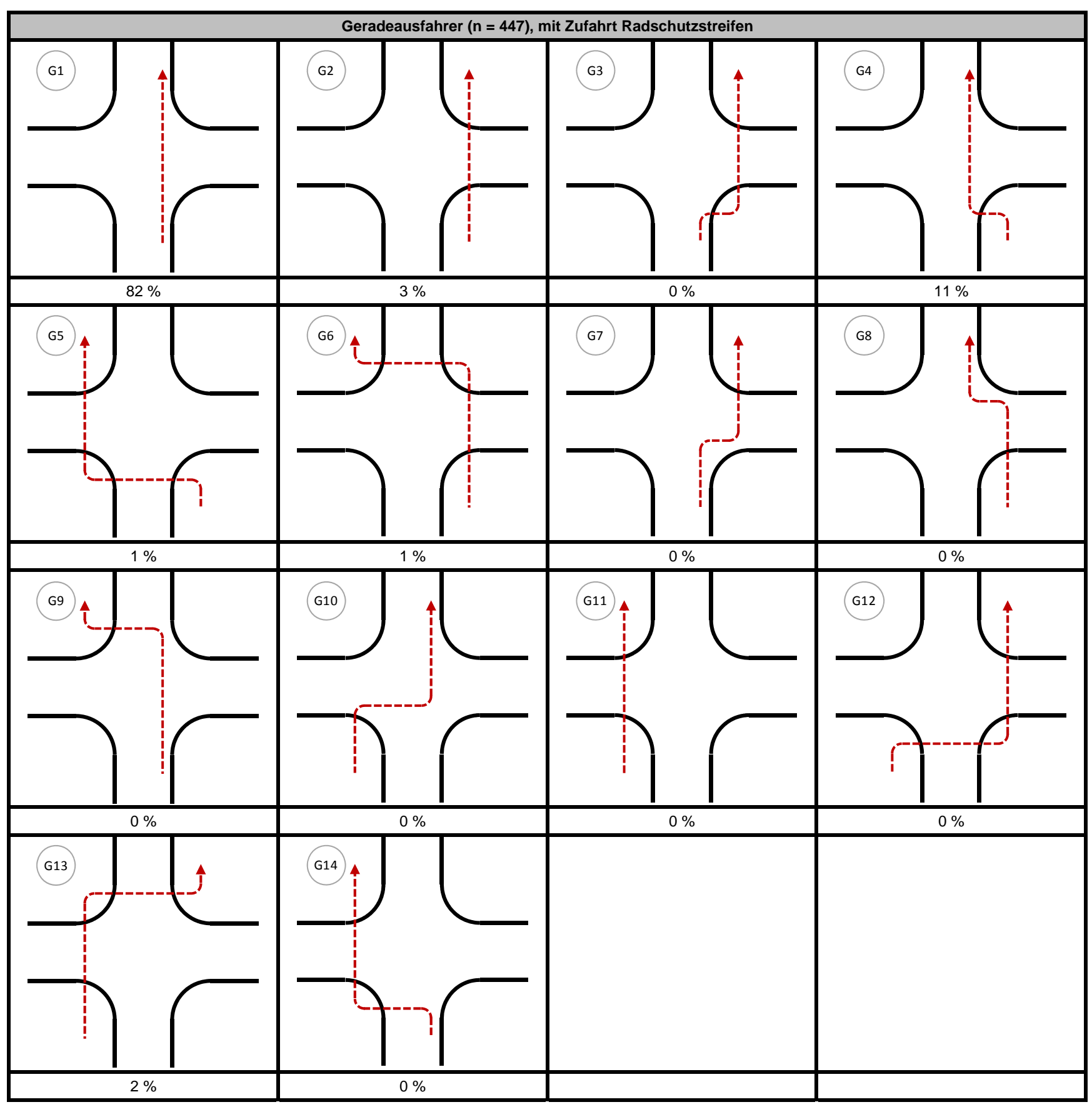


Tabelle 74: Fahrlinien geradeausfahrender Radfahrer mit Führung des Radverkehrs auf der Fahrbahn

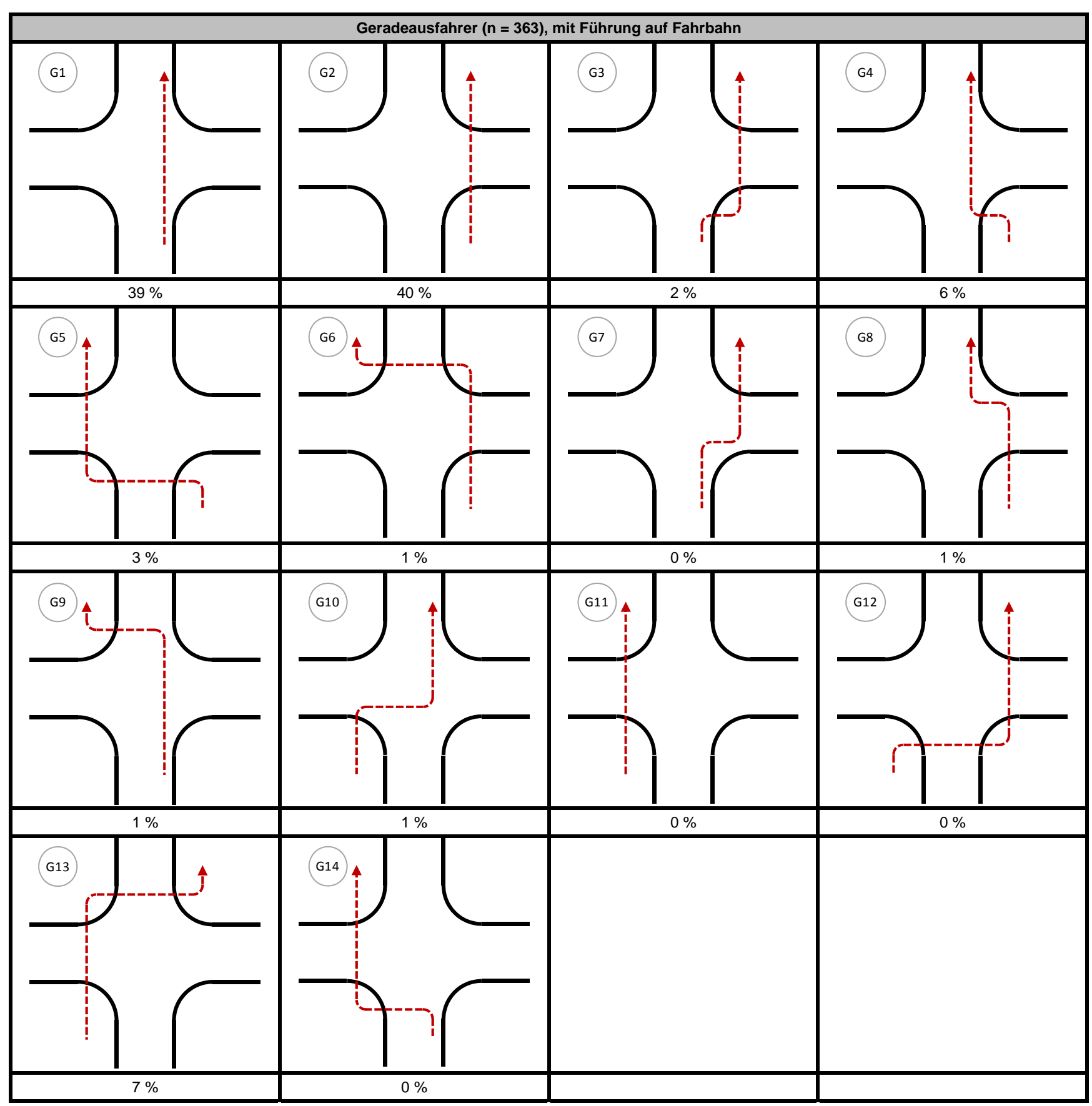




\section{Literatur}

Ahrens, G.-A.; Becker, U.; Böhmer, T.; Richter, F.; Wittwer, R. (2013).: Potenziale des Radverkehrs für den Klimaschutz. Umweltbundesamtes [Hrsg.]. Dessau-Roßlau.

Alrutz, D.; Gündel, D.; Stellmacher-Hein, J.; Lerner, M.; Mättig, W.; Meyhöfer, H.; Angenendt, W.; Draeger, W.; Falkenberg, G.; Klöckner, D.; Abu-Salah, A.; Blase, A.; Rühe, J.; Wilken, M. (2001): Verkehrssicherheit in Einbahnstraßen mit gegengerichtetem Radverkehr. Berichte der Bundesanstalt für Straßenwesen, Heft V 83. Bergisch Gladbach.

Alrutz, D.; Prahlow, H. (2008): Radverkehrssicherheit in Freiburg. Freiburg.

Alrutz, D.; Bohle, W.; Hacke, U.; Lohmann, G.; Müller, H.; Prahlow, H. (2009): Unfallrisiko und Regelakzeptanz von Fahrradfahrern. Bergisch Gladbach: Berichte der Bundesanstalt für Straßenwesen, Heft V 184, Bergisch Gladbach.

Angenendt, W.; Bader, J.; Butz, T.; Cieslik, B.; Draeger, W.; Friese, H.; Klöckner, D.; Lenssen, M.; Wilken, M. (1993): Verkehrssichere Anlage und Gestaltung von Radwegen. Berichte der Bundesanstalt für Straßenwesen, Heft V 9. Bergisch Gladbach.

BASt Forschung kompakt (2011): Volkswirtschaftliche Kosten durch Straßenverkehrsunfälle 2009.

Bertelsmann Stiftung (2011): Deutschland im demographischen Wandel 2030 Datenreport. Gütersloh.

BMVBS [Hrsg.] (2003): Kraftfahrzeugverkehr in Deutschland (KiD) Erhebungsjahr 2002.

BMVBS [Hrsg.] (2004): Mobilität in Deutschland (MiD). Erhebungsjahr 2002.

BMVBS [Hrsg.] (2010): Mobilität in Deutschland (MiD). Erhebungsjahr 2008.

Boenke, D.; Gerlach, J. (2011): Beeinträchtigung im Alter und Empfehlungen zur Gestaltung von Straßenräumen für uns älter werdende Menschen. In: Straßenverkehrstechnik 8/2011.

Cicholas, U.; Ströker, K. (2012): Vorausberechnung der Bevölkerung in den kreisfreien Städten und Kreisen Nordrhein-Westfalens 2011 bis 2030/2050. Information und Technik Nordrhein-Westfalen, Reihe Statistische Analysen und Studien, Band 72. Düsseldorf.

Falkenberg, G.; Blase, A.; Bonfranchi, T.; Cossé, L.; Draeger, W.; Vortisch, P.; Kautzsch, L.; Stapf, H.; Zimmermann, A. (2003): Bemessung von Radverkehrsanlagen unter verkehrstechnischen Gesichtspunkten. Berichte der Bundesanstalt für Straßenwesen, Heft $\checkmark$ 109. Bergisch Gladbach.

FGSV [Hrsg.] (2006): Richtlinien für die Anlage von Stadtstraßen RASt 06. Arbeitsgruppe Straßenentwurf Forschungsgesellschaft für Straßen- und Verkehrswesen. Köln.

FGSV [Hrsg.].(2009): Empfehlungen für Radverkehrsanlagen ERA. Arbeitsgruppe Straßenentwurf Forschungsgesellschaft für Straßen- und Verkehrswesen. Köln.

FGSV [Hrsg.] (2012): Merkblatt zur Örtlichen Unfalluntersuchung in Unfallkommissionen (M Uko). Köln: Arbeitsgruppe Verkehrsmanagement Forschungsgesellschaft für Straßenund Verkehrswesen. Köln.

Funk, W. (2010): Kinder als Radfahrer in der Altersstufe der Sekundarstufe 1. Deutscher Verkehrssicherheitsrat [Hrsg.] Schriftenreihe Verkehrssicherheit, Band 14. Bonn.

Geiler, M.; Pfeiffer, M.; Hautzinger, H. (2007): Das Unfallgeschehen im Wirtschaftsverkehr. Kröning. 
Goth, A. (2005): Untersuchung der Verkehrssicherheit von Radfahrern infolge abbiegender Fahrzeuge unter besonderer Berücksichtigung des Schwerverkehrs an Knotenpunkten (Diplomarbeit), Lehrstuhl für Straßenverkehrstechnik und Theorie der Verkehrsplanung, Technische Universität Dresden. Dresden.

Hannawald, L. (2008): Multivariate Bewertung zukünftiger Fahrzeugsicherheit. s.I. : VDI Verlag $\mathrm{GmbH}$, Bde. Fortschritt-Berichte VDI, Reihe 12, Nr. 682. Düsseldorf.

Hass-Klau, C.; Alrutz, D.; Crampton, G.; Booking, T.; Nold, I.; Trush, J.; Lüers, A.; Brückner, T.; Frommherz, W.; Meyhöfer, H.; Rohlfing, M. (1992): Sicherheit des Fahrradverkehrs. ein Vergleich zwischen deutschen und britischen Städten. Bericht zum Forschungsprojekt 8747 der Bundesanstalt für Straßenwesen. Bergisch-Gladbach.

Hautzinger, H. (1993): Dunkelziffer bei Unfällen mit Personenschaden. Berichte der Bundesanstalt für Straßenwesen, Heft M 13. Bergisch Gladbach.

Hautzinger, H.; Tassaux-Becker, B.; Hamacher, R. (1996): Verkehrsunfallrisiko in Deutschland. Verkehrsmobilität in Deutschland zu Beginn der 90er Jahre, Band 5. Berichte der Bundesanstalt für Straßenwesen, Heft M 58. Bergisch-Gladbach.

Hutcheson, G.D.; Sofroniou, N. (1999): The Multivariate Social Scientist: Introductory Statistics Using Generalized Linear Models. London.

Kolrep-Rometsch, H.; Leitner, R.; Platho, C.; Richter, T.; Schreiber, A.; Schreiber, M.; Butterwegge, P. (2013): Abbiegeunfälle Pkw/Lkw und Fahrrad. Forschungsberichte Nr. 21 der UDV. Berlin.

Lawinger, T. (2012): Neue Formen der Zweiradmobilität. Eine empirische Tiefenanalyse auf Grundlage von Daten aus Baden-Württemberg. Masterarbeit. Deutsche Hochschule der Polizei.

Limbourg, M. (2008): Kinder unterwegs im Straßenverkehr. Reihe Prävention in NRW der Unfallkassen NRW, Nr. 12. Düsseldorf.

Landesbetrieb für Statistik und Kommunikationstechnologie Niedersachsen (LSKN) (2011): Die Ergebnisse der regionalen Bevölkerungsvorausberechnung für Niedersachsen bis zum 01.01.2031 - Basis 2009 -. Hannover.

Maier, R.; Aurich, A.; Schüller, H. (2010): Sicherheitskonzept Dresden. Dresden. 2010. unveröffentlicht

Mullins, N.C. (1974): Theory Constructions From Available Materials. A System for Organizing and Presenting Propositions. In: American Journal of Sociology 80. Chicago

Ortlepp, J.; Neumann, V.; Utzmann, I. (2008): Verbesserung der Verkehrssicherheit in Münster. Unfallforschung der Versicherer. Berlin.

Otte, D.; Haasper, C.; Wiese, B. (2008): Wirksamkeit von Fahrradhelmen bei Verkehrsunfällen von Radfahrern auf Kopfverletzungshäufigkeit und Verletzungsschwere. In: Verkehrsunfall und Fahrzeugtechnik, Ausgabe Oktober. München.

Richter, C. (2011): Erstellung von Verletzungsrisikofunktionen für verschiedene Verkehrsbeteiligungsarten und Anprallkonstellationen (Diplomarbeit). Lehrstuhl für Straßenverkehrstechnik und Theorie der Verkehrsplanung, Technische Universität Dresden. Dresden.

Ruwenstroth, G. Radder, F. (1991): Wirksamkeitskontrolle kommunaler Verkehrssicherheitsmaßnahmen - Fahrradstraße Hansestadt Lübeck. Forschungsberichte der Bundesanstalt für Straßenwesen, Heft 225. Bergisch-Gladbach.

Schepers, P.; Agerholm, N.; Amoros, E.; Benington, R.; Bjørnskau, T.; Dhondt, S.; de Geus, B.; Hagemeister, C.; Loo, B.; Niska, A. (2014): An international review of the frequency 
of single-bicycle crashes (SBCs) and their relation to bicycle modal share. Delft, Niederlande.

Schiller, C.; Zimmermann, F.; Bohle, W. (2011): Hochrechnungsmodell von Stichprobenzählungen für den Radverkehr. Excel-Tool und Bericht zu dem FE-Vorhaben 77.495 des BMVBS. Dresden.

Schnüll, R.; Alrutz, D.; Lange, J.; Fabian, I.; Kölle, M.; Schütte, F.; Fechtel, H.; StellmacherHein, J.; Brückner, T.; Meyhöfer, H. (1992): Sicherung von Radfahrern an städtischen Knotenpunkten. Bericht zum Forschungsprojekt 8925 der Bundesanstalt für Straßenwesen. Bergisch-Gladbach.

Schopf, J. (1992): Beiträge zu einer ökologisch und sozial verträglichen Verkehrsplanung: Die Geschwindigkeit im Straßenverkehr, Institut für Verkehrsplanung und Verkehrstechnik der Technischen Universität Wien. Wien.

Schmitz-Veltin, A. (2009): Einwohnerprognose 2009 bis 2025. Die Entwicklung der Zahl der Einwohner in den Stuttgarter Stadtbezirken. Statistisches Amt der Landeshauptstadt Stuttgart. Stuttgart.

SrV. System repräsentativer Verkehrserhebungen. (2009): Erhebungsjahr 2008. Technische Universität Dresden. Dresden.

Statistisches Bundesamt (versch. Jahre): Verkehrsunfälle, Fachserie 8 Reihe 7. Wiesbaden.

Statistisches Bundesamt (2009): Bevölkerung Deutschlands bis 2060 - 12. koordinierte Bevölkerungsvorausberechnung. Wiesbaden.

Statistisches Bundesamt (2012): Statistisches Jahrbuch 2011. Wiesbaden.

Statistisches Bundesamt (2014):. Vorläufige Ergebnisse der Bevölkerungsfortschreibung 2011 auf Grundlage des Zensus 2011. Wiesbaden.

Steffens, U.; Pfeifer, K.; Schreiber, N.; Rudinger, G.; Groß, H.; Hübner, G. (1999): Ältere Menschen als Radfahrer. Berichte der Bundesanstalt für Straßenwesen; Heft M 112. Bergisch-Gladbach.

Stiftung Warentest (2011): Bremsen oft zu schwach. http://www.test.de/themen/freizeitreise/test/Elektrofahrraeder-Bremsen-oft-zu-schwach-4260019-4261622/ vom 12.9.2011.

UDV (2011a): Elektrofahrräder: Umweltschonend aber gefährlich? Vortragsmanuskript S. Brockmann. Neumünster

UDV (2011b): Fahrradunfallstudie Münster. Münster.

UDV (2014): Neues Risiko Pedelec?, Unfallforschung kompakt Nr. 46, Unfallforschung der Versicherer (UDV) im Gesamtverband der Deutschen Versicherungswirtschaft e. V. (GDV). Berlin.

Van Boggelen, O.; van Oijen, J. (2013): Verder met de e-fiets, In: Fietsverkeer, Heft 32. Utrecht.

Verwaltungsvorschrift zur Straßenverkehrs-Ordnung (VwV-StVO) (2009).

Zweirad-Industrie-Verband (ZIV) (2013): Zahlen - Daten - Fakten zum Fahrradmarkt in Deutschland und Europa. ZIV Wirtschaftspressekonferenz. Berlin.

Walker, I. (2007): Drivers overtaking bicyclists: Objective data on the effects of riding position, helmet use, vehicle type and apparent gender. Accident Analysis and Prevention 39. 


\section{Abbildungsverzeichnis}

Bild 1: Entwicklung der Zahl schwer verletzter oder getöteter Radfahrer (Statistisches

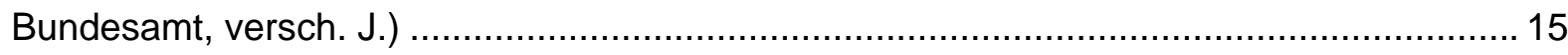

Bild 2: Entwicklung der Zahl leicht verletzter Radfahrer (Statistisches Bundesamt, versch. J.)....... 15

Bild 3: Geschwindigkeitsverteilung der Radfahrer im Berufs- und Freizeitverkehr ( $N=150$ je Stichprobe, Altersverteilung jeweils vergleichbar mit Tabelle 3) (Schopf, 1992) .................. 24

Bild 4: Häufigkeiten der beeinflussten Geschwindigkeiten (Falkenberg et al., 2003) ................... 25

Bild 5: Verletzungsrisikofunktionen für Fahrradfahrer unter Einfluss des Alters (Richter, 2011) ..... 26

Bild 6: Struktogramm der Arbeitsschritte.................................................................... 30

Bild 7: gemessene Geschwindigkeiten des Radverkehrs (mittlere Geschwindigkeit $v_{m}, 15 \%$ Quantil $v_{15}, 50 \%$-Quantil v50 und $85 \%$-Quantil $v_{85}$ sowie Minimum $v_{\min }$ und Maximum

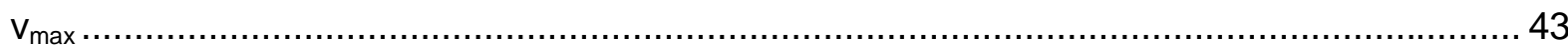

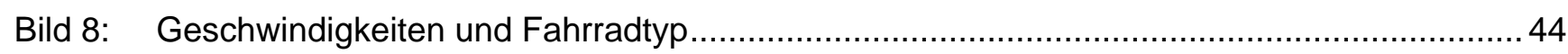

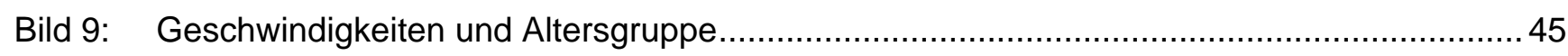

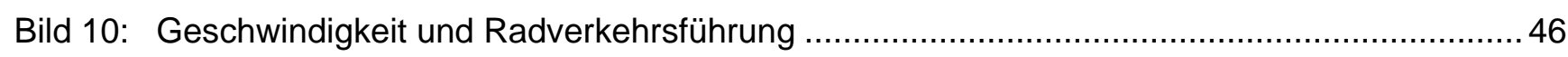

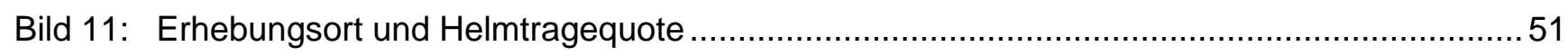

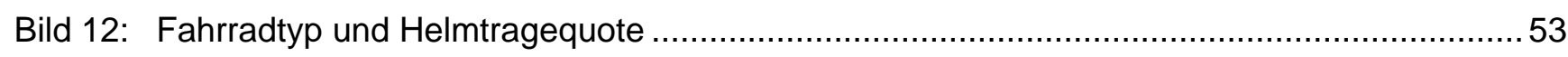

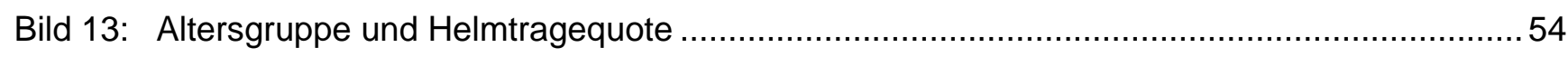

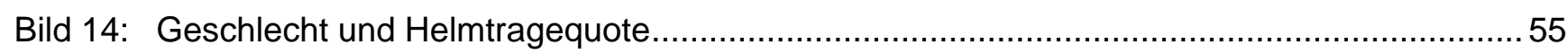

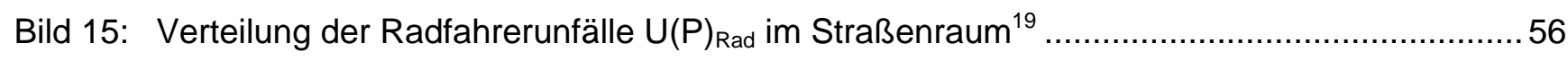

Bild 16: Unfallzahlen $U(P)_{\text {Rad }} / 3 a$, Verkehrsstärken $\mathrm{Rad} / 24 \mathrm{~h}$ und Führungsformen des

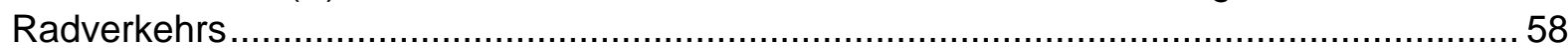

Bild 17: Modellparameter mit systematischem Einfluss auf die Radverkehrsunfallanzahl $U(P)_{\operatorname{Rad}} \ldots 60$

Bild 18: Unfallraten des heutigen Radverkehrs nach Anlagentypen $(\mathrm{n}=$ Anzahl Streckenabschnitte) .

Bild 19: Radverkehrsstärken auf den zuführenden Streckenabschnitten lichtsignalgeregelter Knotenpunkte und Anzahl von Radfahrer-Unfällen mit Personenschaden in drei Jahren (beide Fahrtrichtungen, alle Unfallorte.....

Bild 20: Radverkehrsstärken auf den zuführenden Streckenabschnitten lichtsignalgeregelter Knotenpunkte und Anzahl von Radfahrer-Unfällen mit Personenschaden in drei Jahren (rechte Fahrtrichtung, Unfallort auf vorgesehener Radverkehrsführung).

Bild 21: Zusammenhänge zwischen Verkehrsaufkommen, Verkehrsleistung und

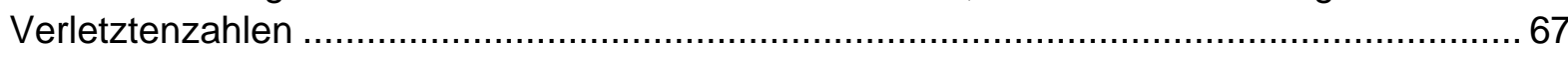

Bild 22: Unfallschwere nach Radverkehrsstärke in zwei Klassen ............................................69

Bild 23: Radverkehrsstärken und Unfallkostendichte auf straßenbündigen Radverkehrsführungen (Mischverkehr, Radfahrstreifen, Schutzstreifen, Bussonderfahrstreifen). 
Bild 24: Radverkehrsstärken und Unfallkosten je Unfall auf straßenbündigen Radverkehrsführungen (Mischverkehr, Radfahrstreifen, Schutzstreifen,

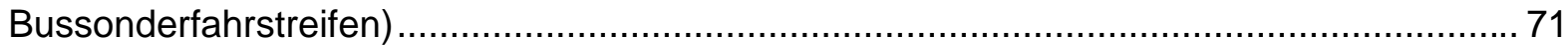

Bild 25: Radverkehrsstärke und Anteil von Unfällen mit schwerem Personenschaden an allen Unfällen mit Personenschaden an lichtsignalgeregelten Knotenpunkten (beide Fahrtrichtungen, alle Unfallorte, einschließlich unfallfreie Knotenzufahrten)

Bild 26: Radverkehrsstärke und Anteil von Unfällen mit schwerem Personenschaden an allen Unfällen mit Personenschaden an lichtsignalgeregelten Knotenpunkten (rechte Fahrtrichtung, Unfallort: vorgesehen Radverkehrsführung, einschließlich unfallfreie Knotenzufahrten).

Bild 27: Radverkehrsstärken auf den zuführenden Streckenabschnitten und Unfallschwere an lichtsignalgeregelten Knotenpunkten (beide Fahrtrichtungen, alle Unfallorte).

Bild 28: Radverkehrsstärken auf den zuführenden Streckenabschnitten und Unfallschwere an lichtsignalgeregelten Knotenpunkten (rechte Fahrtrichtung und Unfallorte auf der vorgesehenen Radverkehrsfläche)

Bild 29: Mittlere Unfallrate nach Führungsform an lichtsignalgeregelten Knotenpunkten..... 76

Bild 30: Mittlere Unfallkostenrate nach Führungsform an lichtsignalgeregelten Knotenpunkten ....... 76

Bild 31: Unfalltypen (Anteile, ohne Typ 7) und Radverkehrsstärke in zwei Klassen

Bild 32: Anteile der rechten und regelwidrigen linken Fahrtrichtung nach Anlagentyp (ohne Differenzierung nach Flächennutzung)

Bild 33: Radverkehrsstärken und Anteil regelwidrig links Fahrender in Straßen mit benutzungspflichtigen Radwegen, alle Städtebaulichen Nutzungen

Bild 34: Radverkehrsstärken und Anteil regelwidrig links Fahrender in Straßen mit benutzungspflichtigen Radwegen, Einzelhandels- und Mischnutzungen

Bild 35: Radverkehrsstärken und Anteil regelwidrig links Fahrender in Straßen mit benutzungspflichtigen Radwegen. Wohn, Gewerbe- und Sonstige Nutzungen ohne Einzelhandels- und Mischnutzungen

Bild 36: Unfallraten in linker und rechter Fahrtrichtung

Bild 37: Anzahl regelwidrig links Fahrender und Unfalldichte links Fahrender in Straßen mit benutzungspflichtigen Radwegen

Bild 38: Anzahl regelwidrig links Fahrender auf Radweg und Unfalldichte links Fahrender auf Radweg in Straßen mit benutzungspflichtigen Radwegen (69 Straßenseiten, $65 \mathrm{U}(P)$ ) ......87

Bild 39: Radverkehrsstärke in rechter Richtung und Anzahl Gehwegnutzer in rechter Richtung ...... 88

Bild 40: Radverkehrsstärke in rechter Richtung und Anteil Gehwegnutzer in rechter Richtung ........ 89

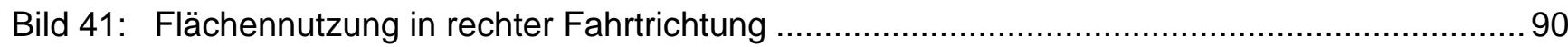

Bild 42: Anteile der Fahrtrichtungen auf den Groninger Streckenabschnitten .............................91

Bild 43: Anzahl Radfahrer und Anteil regelwidrig links Fahrender auf benutzungspflichtigen Radwegen in Groningen

Bild 44: benutzungspflichtiger Radweg mit Höhentrennung zu Gehweg in Groningen 92

Bild 45: Flächennutzung in rechter Richtung auf den Groninger Untersuchungsabschnitten 92

Bild 46: Anzahl beobachteter Radfahrer in der Zufahrt und Anteil der Rotfahrer bei Führung auf Fahrbahnen, Radwegen, Radfahrstreifen und Schutzstreifen 
Bild 47: Anzahl beobachteter Radfahrer in der Zufahrt und Anteil der Unfälle in der Zufahrt mit „Nichtbeachten der Verkehrsregelung durch Lichtzeichen“ als Unfallursache.

Bild 48: Unfallrate und Verkehrsbeteiligung auf den untersuchten Streckenabschnitten nach Altersgruppe der Radfahrer.

Bild 49: Anteile der Unfälle mit Über-65-Jährigen und Anteil der Über-65-Jährigen am Radverkehr auf zuführendem Streckenabschnitt

Bild 50: Unfallschwere nach Alter der Radfahrer und Verursachung auf Streckenabschnitten (erweitertes Unfallkollektiv)

Bild 51: Unfallschwere nach Alter der Radfahrer und Verursachung an Verkehrsknotenpunkten mit LSA (erweitertes Unfallkollektiv).

Bild 52: Unfalltypen (Anteile, ohne Typ 7) nach Alter und Unfallverursachung an Strecken (erweitertes Unfallkollektiv)

Bild 53: Unfalltypen (Anteile, ohne Typ 7) nach Alter und Unfallverursacher an Knotenpunkten (erweitertes Unfallkollektiv)

Bild 54: Regelwidrige Nutzung linker Furten nach Altersgruppe an lichtsignalgeregelten Knotenpunkten.....

Bild 55: Radgeschwindigkeiten in $\mathrm{v}_{85}$ und Unfallanzahl 105

Bild 56: Radverkehrsstärke in Radverkehrsstärkeklassen und Radgeschwindigkeiten in $\mathrm{v}_{85}$...... 107

Bild 57: Nennung der Unfallursache 12 und 13 „nicht angepasste Geschwindigkeit“ und Unfallanzahl

Bild 58: Unfallstruktur und $\mathrm{v}_{85}$ der Radfahrer in zwei Klassen.

Bild 59: Radfahrergeschwindigkeit (gefahrene $v_{85}$ (klassifiziert)) und Unfallschwere (Anteil schwerer Radverkehrsunfälle (schwerer Personenschaden) an allen Radverkehrsunfällen mit Personenschaden)

Bild 60: Unfalltypen (Anteile, ohne Typ 7) und $\mathrm{v}_{85}$ der Radfahrer in zwei Klassen.

Bild 61: Nennung der Unfallursache 12 und 13 „nicht angepasste Geschwindigkeit“ und Unfallschwere.

Bild 62: Ablauf der Szenarienberechnungen für Strecken

Bild 63: Inhaltlicher Ablauf der Szenarienberechnungen zur Aufteilung der Unfälle auf die Streckenabschnitte.

Bild 64: Ergebnisse der Szenarienberechnung zur Unfallaufteilung an Streckenabschnitten

Bild 65: Beispiel eines Q-Q-Plots

Bild 66: Beispiel eines Residuenplots über die Einflussgröße DTV

Bild 67: Beispiel Cure-Plot über die Einwohneranzahl und Histogramm der logarithmierten Einwohnerzahl.

Bild 68: Beispielparametersatz......

Bild 69: Führungsformen und Radverkehrsstärke in zwei Klassen.

Bild 70: Unfälle nach Führungsformen und Radverkehrsstärke in zwei Klassen. 152

Bild 71: Unfalltypen (Anteile, mit Typ 7) und Radverkehrsstärke in zwei Klassen . 153

Bild 72: Unfalltypen nach Führungsformen und Radverkehrsstärke in zwei Klassen 
Bild 73: Unfalltypen (Anteile, mit Typ 7), Verursachung und Alter in zwei Klassen ....................... 155

Bild 74: Unfalltypen an Strecken (Anteile, mit Typ 7) und Geschwindigkeit in zwei Klassen ........... 156

Bild 75: Unfalltypen an Knotenpunkten (Anteile, mit Typ 7), Verursachung und Alter in zwei

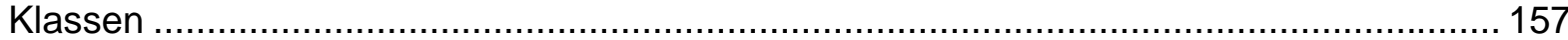

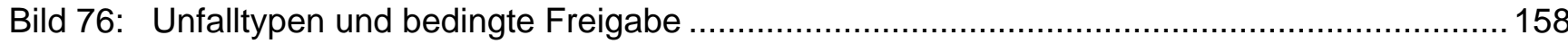

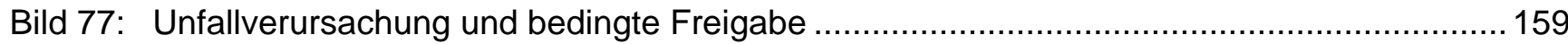

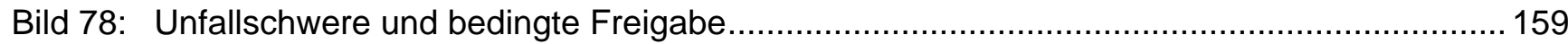




\section{Tabellenverzeichnis}

Tabelle 1: Verunglückte Radfahrer nach Alter und Geschlecht 2011 (Statistisches Bundesamt, 2012)

Tabelle 2: Verletzungsrisiko nach Altersgruppen bei Radfahrern im Wirtschaftsverkehr (Geiler, 2007).....

Tabelle 3: Durchschnittsgeschwindigkeiten von Radfahrern nach Altersgruppen (Schopf, 1992) .. 24

Tabelle 4: Anzahl der Untersuchungsstrecken in den deutschen Städten (Straßenseiten)............. 32

Tabelle 5: $\quad$ Führung des Radverkehrs in den Knotenzufahrten (Anzahl der Knotenzufahrten) ......... 32

Tabelle 6: Übersicht der aufgenommenen Variablen (baulich-betriebliche Merkmale und

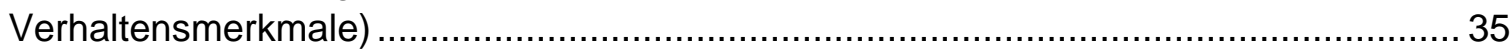

Tabelle 7: $\quad$ Übersicht der aufgenommenen Variablen (Unfallanalysen)........................................ 36

Tabelle 8: Hypothesen zur Entwicklung der Radverkehrssicherheit ........................................... 39

Tabelle 9: Untersuchte Einflüsse und Gliederung zur Darstellung der Untersuchungsergebnisse

Tabelle 10: Altersgruppen der gezählten Radfahrer und Anteile der Altersgruppen an der Verkehrsleistung des Radverkehrs (eigene Berechnung auf Grundlage der Stichprobe der MiD 2008)

Tabelle 11: Gezählte und in Betrieb befindliche Fahrradtypen (ZIV, 2013)

Tabelle 12: Altersgruppen der Radfahrer bei den Geschwindigkeitsmessungen und Anteile der Altersgruppen an der Verkehrsleistung des Radverkehrs (Eigene Berechnung auf Grundlage der korrigierten Stichprobe der MiD 2008)

Tabelle 13: Signifikanztest: Geschwindigkeiten und Fahrradtypen

Tabelle 14: Signifikanztest: Geschwindigkeiten nach Altersgruppen......

Tabelle 15: Signifikanztest: Geschwindigkeiten nach Radverkehrsführung

Tabelle 16: mittlere Abweichung der Geschwindigkeiten nach konstant gehaltenen und unabhängigen Variablen

Tabelle 17: Häufige Fahrlinien linksabbiegender Radfahrer nach Radverkehrsführung

Tabelle 18: Häufige Fahrlinien linksabbiegender Radfahrer nach Furtabsatzung bei Radwegen ..... 49

Tabelle 19: Häufige Fahrlinien linksabbiegender Radfahrer bei Fahrbahnführung 50

Tabelle 20: Häufige Fahrlinien geradeausfahrender Radfahrer nach Furtabsetzung bei Radwegen. 50

Tabelle 21: Signifikanztest: Helmtragequote nach Erhebungsort 52

Tabelle 22: Signifikanztest: Helmtragequote und Fahrradtypen ${ }^{18}$ .53

Tabelle 23: Signifikanztest: Helmtragequote und Altersgruppe .54

Tabelle 24: Signifikanztest: Helmtragequote und Geschlecht 55

Tabelle 25: Flächennutzung verunglückter Radfahrer (Anzahl U (P)) 62

Tabelle 26: Verkehrsanteile des Radverkehrs und Anteil an den Verletzten in den einzelnen Städten 
Tabelle 27: Anteile der Unfalltypen nach Radverkehrsanlage und Radverkehrsstärkenklasse an Streckenabschnitten

Tabelle 28: Unfalltypen nach Führung in der Knotenzufahrt und Radverkehrsstärkeklasse (Anteile nach Führungsform und Verkehrsstärke)

Tabelle 29: Beobachtete Fahrtrichtungen (alle Streckenabschnitte)

Tabelle 30: Beobachtete Fahrtrichtungen und Fahrtrichtungen bei Unfällen nach Altersgruppe (Streckenabschnitte der Mikroanalyse).

Tabelle 31: Flächennutzung nach Fahrtrichtung und Altersgruppe bei benutzungspflichtigen Radwegen (Anteil nach Altersgruppe in \%)

Tabelle 32: Flächennutzung nach Fahrtrichtung und Altersgruppe bei nicht benutzungspflichtigen Radwegen (Anteil nach Altersgruppe in \%).

Tabelle 33: Flächennutzung nach Fahrtrichtung und Altersgruppe bei Mischverkehr (Anteil nach Altersgruppe in \%)

Tabelle 34: Flächennutzung nach Fahrtrichtung und Altersgruppe in Straßen mit Radfahrstreifen (Anteil nach Altersgruppe in \%).

Tabelle 35: Flächennutzung nach Fahrtrichtung und Altersgruppe in Straßen mit Schutzstreifen (Anteil nach Altersgruppe in \%).

Tabelle 36: separates Unfallmodel zur Ermittlung des Einflusses der Geschwindigkeiten im Radverkehr.....

Tabelle 37: Unfalltypen nach Anlagentyp und Radfahrergeschwindigkeit in zwei Klassen

Tabelle 38: Übersicht der ermittelten Einflüsse aus Radverkehrsstärke, demografischer Wandel und Radfahrergeschwindigkeit auf die Anzahl, Schwere und Typen der Radverkehrsunfälle

Tabelle 39: Übersicht der Entwicklungstendenzen in den Szenarien ....

Tabelle 40: Grundlagen des Szenarios S1 „Moderate Zunahme des Radverkehrs“

Tabelle 41: Grundlagen des Szenarios S1 „Moderate Zunahme des Radverkehrs“

Tabelle 42: Grundlagen des Szenarios S2-A „Veränderung der Altersstruktur des Radverkehrs".....

Tabelle 43: Grundlagen des Szenarios S2-A-20 „Veränderung der Altersstruktur und moderate Zunahme des Radverkehrs".

Tabelle 44: Grundlagen des Szenarios S2-A-20-V „Veränderung der Geschwindigkeiten aufgrund einer veränderten Altersstruktur und veränderter Fahrradtypen bei moderater Zunahme der Radverkehrsstärken“.

Tabelle 45: Grundlagen „Erheblich höhere Stärken des Radverkehrs“

Tabelle 46: Grundlagen des Szenarios S2-A-40 „Veränderung der Altersstruktur und erheblich höhere Stärken des Radverkehrs"....

Tabelle 47: Grundlagen des Szenarios S2-A-40-V „Erheblich höhere Stärken und Veränderung der Geschwindigkeiten des Radverkehrs“

Tabelle 48: Ergebnisse der Szenarienberechnung zur Unfallschwere an Streckenabschnitten...... 130

Tabelle 49: Ergebnisse der Szenarien zur Verteilung der Unfalltypen an Streckenabschnitten ...... 133

Tabelle 50: Ergebnisse der Szenarien zur Zunahme der Unfalltypen an Streckenabschnitten. 
Tabelle 51: Ergebnisse der Szenarienberechnung zur Unfallanzahl und -schwere an lichtsignalgeregelten Knotenpunkten ....

Tabelle 52: Ergebnisse der Szenarien zur Entwicklung der Unfalltypen an lichtsignalgeregelten Knotenpunkten

Tabelle 53: Verteilung der heutigen Unfälle auf das Untersuchungskollektiv an Streckenabschnitten und lichtsignalgeregelten Knotenpunkten.

Tabelle 54: Untersuchte Modellparameter.

Tabelle 55: angepasste Unfallkostensätze der Radfahrunfälle (nach Preisstand 2009)

Tabelle 56: Beispiel für die Ermittlung einer AD-Streuung..... 160

Tabelle 57: Fahrlinien linksabbiegender Radfahrer mit Radwegzufahrt 161

Tabelle 58: Fahrlinien linksabbiegender Radfahrer mit Radwegzufahrt bei nicht abgesetzter Furt.......

Tabelle 59: Fahrlinien linksabbiegender Radfahrer mit Radwegzufahrt bei abgesetzter Furt

Tabelle 60: Fahrlinien linksabbiegender Radfahrer mit zuführendem Radfahrstreifen..... 164

Tabelle 61: Fahrlinien linksabbiegender Radfahrer mit zuführendem Radschutzstreifen. 165

Tabelle 62: Fahrlinien linksabbiegender Radfahrer mit Führung des Radverkehrs auf der Fahrbahn 166

Tabelle 63: Fahrlinien rechtsabbiegender Radfahrer mit Radwegzufahrt. 167

Tabelle 64: Fahrlinien rechtsabbiegender Radfahrer mit Radwegzufahrt bei nicht abgesetzter Furt.

Tabelle 65: Fahrlinien rechtsabbiegender Radfahrer mit Radwegzufahrt bei abgesetzter Furt 169

Tabelle 66: Fahrlinien rechtsabbiegender Radfahrer mit zuführendem Radfahrstreifen 170

Tabelle 67: Fahrlinien rechtsabbiegender Radfahrer mit zuführendem Radschutzstreifen 171

Tabelle 68: Fahrlinien rechtsabbiegender Radfahrer mit Führung des Radverkehrs auf der Fahrbahn.

Tabelle 69: Fahrlinien geradeausfahrender Radfahrer mit Radwegzufahrt

Tabelle 70: Fahrlinien geradeausfahrender Radfahrer mit Radwegzufahrt bei nichtabgesetzter Furt

Tabelle 71: Fahrlinien geradeausfahrender Radfahrer mit Radwegzufahrt bei abgesetzter Furt .... 175

Tabelle 72: Fahrlinien geradeausfahrender Radfahrer mit zuführendem Radfahrstreifen. 176

Tabelle 73: Fahrlinien geradeausfahrender Radfahrer mit zuführendem Radschutzstreifen 177

Tabelle 74: Fahrlinien geradeausfahrender Radfahrer mit Führung des Radverkehrs auf der Fahrbahn. 


\section{GDV}

DIE DEUTSCHEN VERSICHERER

\section{Gesamtverband der Deutschen Versicherungswirtschaft e. V.}

Wilhelmstraße 43 / 43 G, 10117 Berlin

Postfach 0802 64, 1002 Berlin

Tel. $030 / 2020$-50 00, Fax 030 / 2020 - 6000

www.gdv.de, www.udv.de 